\title{
On the Numerical Solution of Laplace's Equation with Nonlinear Boundary Conditions for Corrosion of Steel in Concrete
}

by

\author{
Ge Ji, B.Sc.
}

A thesis submitted to the Faculty of Graduate Studies and Research

in partial fulfillment of requirements for the degree of

\section{Master of Applied Science}

Department of Civil and Environment Engineering

Carleton University

Ottawa, Ontario, Canada

The Master of Applied Science in Civil Engineering is a joint program with the

University of Ottawa, administered by the Ottawa-Carleton Institute for Civil Engineering

(C) 2006, Ge Ji 


$\begin{array}{ll}\begin{array}{l}\text { Library and } \\ \text { Archives Canada }\end{array} & \begin{array}{l}\text { Bibliothèque et } \\ \text { Archives Canada }\end{array} \\ \begin{array}{l}\text { Published Heritage } \\ \text { Branch }\end{array} & \begin{array}{l}\text { Direction du } \\ \text { Patrimoine de l'édition }\end{array} \\ \begin{array}{l}\text { 395 Wellington Street } \\ \text { Ottawa ON K1A ON4 }\end{array} & \begin{array}{l}\text { 395, rue Wellington } \\ \text { Ottawa ON K1A ON4 } \\ \text { Canada }\end{array}\end{array}$

Your file Votre référence ISBN: 978-0-494-18317-5 Our file Notre référence ISBN: 978-0-494-18317-5

NOTICE:

The author has granted a nonexclusive license allowing Library and Archives Canada to reproduce, publish, archive, preserve, conserve, communicate to the public by telecommunication or on the Internet, loan, distribute and sell theses worldwide, for commercial or noncommercial purposes, in microform, paper, electronic and/or any other formats.

The author retains copyright ownership and moral rights in this thesis. Neither the thesis nor substantial extracts from it may be printed or otherwise reproduced without the author's permission.
AVIS:

L'auteur a accordé une licence non exclusive permettant à la Bibliothèque et Archives Canada de reproduire, publier, archiver, sauvegarder, conserver, transmettre au public par télécommunication ou par l'Internet, prêter, distribuer et vendre des thèses partout dans le monde, à des fins commerciales ou autres, sur support microforme, papier, électronique et/ou autres formats.

L'auteur conserve la propriété du droit d'auteur et des droits moraux qui protège cette thèse. $\mathrm{Ni}$ la thèse ni des extraits substantiels de celle-ci ne doivent être imprimés ou autrement reproduits sans son autorisation.
In compliance with the Canadian

Privacy Act some supporting forms may have been removed from this thesis.

While these forms may be included in the document page count, their removal does not represent any loss of content from the thesis.
Conformément à la loi canadienne sur la protection de la vie privée, quelques formulaires secondaires ont été enlevés de cette thèse.

Bien que ces formulaires aient inclus dans la pagination, il n'y aura aucun contenu manquant.

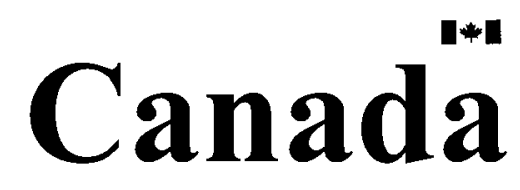




\begin{abstract}
To overcome the difficulties associated with the solution of Laplace's equation for electric potentials with nonlinear polarized boundary conditions, an improved two-dimensional nonlinear finite element technique was developed to model steel corrosion in concrete members. The nonlinear solution algorithm was implemented as a Windows-based computer program using Visual Basic.Net 2003. The program uses a finite element engine that was previously developed at Carleton University, Condur, to solve Laplace's equation. With the new approach, the convergence rate and the stability of the nonlinear solution are significantly improved. The sensitivity of the results and convergence of the solution with respect to element size, anode-to-cathode ratio and concrete resistivity are investigated. The effect of the selection of corrosion parameters on the solution is also studied.
\end{abstract}




\section{TABLE OF CONTENTS}

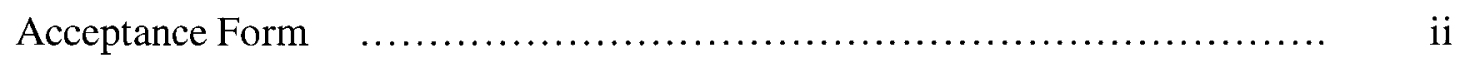

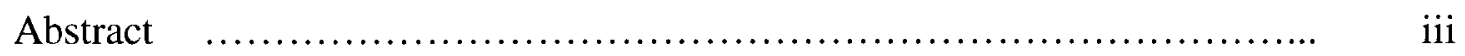

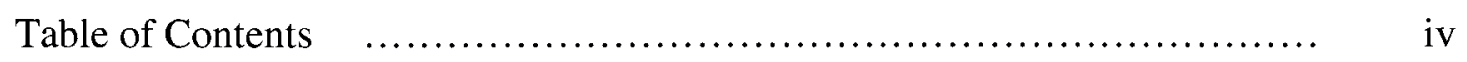

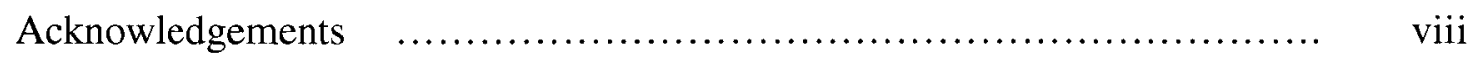

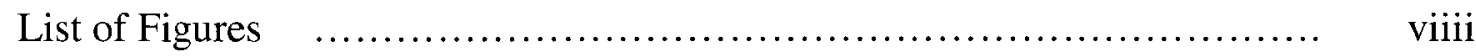

List of Tables $\quad$........................................................ xviii

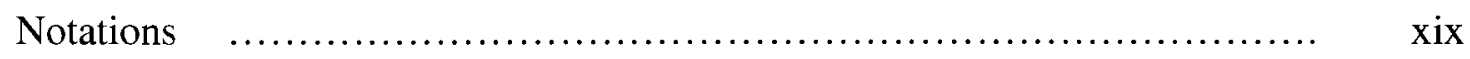

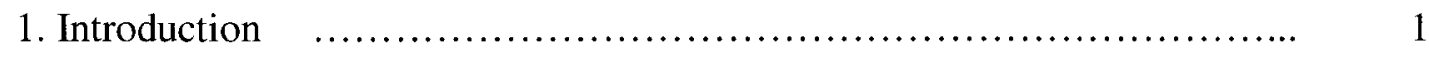

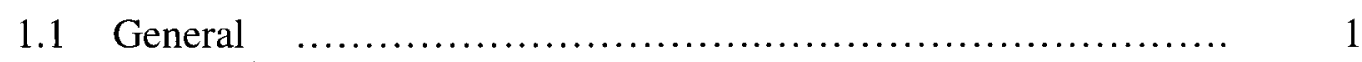

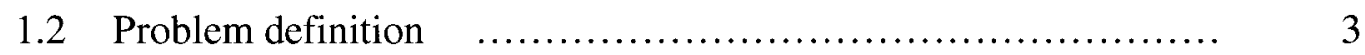

1.3 Objectives and scope of the research $\quad \ldots \ldots \ldots \ldots \ldots \ldots \ldots \ldots \ldots \ldots, 4$

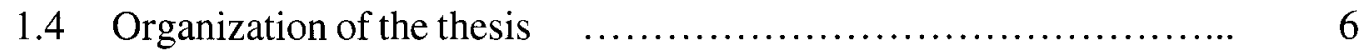

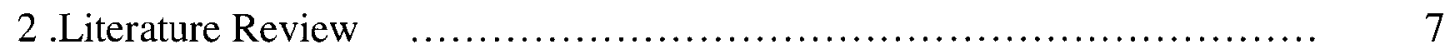

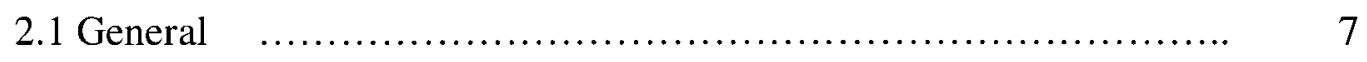

2.2 Modelling steel corrosion in concrete $\quad \ldots \ldots \ldots \ldots \ldots \ldots \ldots \ldots \ldots \ldots . \ldots \ldots$

2.3 Summary of the literature review $\quad \ldots \ldots \ldots \ldots \ldots \ldots \ldots \ldots \ldots \ldots \ldots . \ldots \ldots$

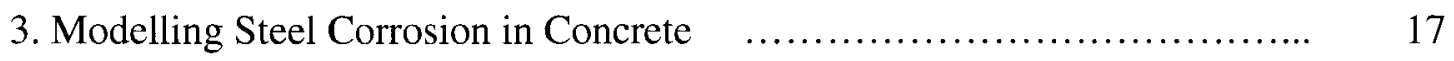

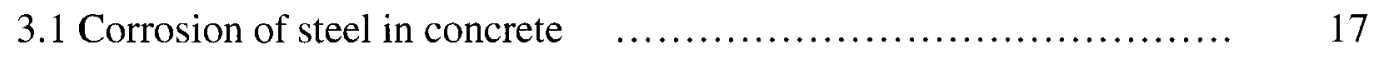

3.1.1 Electrochemical process $\quad \ldots \ldots \ldots \ldots \ldots \ldots \ldots \ldots \ldots \ldots \ldots \ldots . \ldots \ldots \ldots \ldots$

iv 


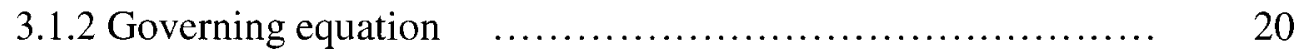

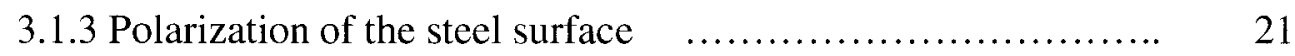

3.2 Modelling corrosion of steel in concrete $\quad \ldots \ldots \ldots \ldots \ldots \ldots \ldots \ldots . . \ldots . \ldots . \ldots 26$

3.2.1 Dirichlet-Type Boundary Definition $\quad \ldots \ldots \ldots \ldots \ldots \ldots \ldots . \ldots . \ldots . \ldots 27$

3.2.2 Mixed Dircihlet/Neumann Boundary Definition $\quad \ldots \ldots \ldots \ldots . . .28$

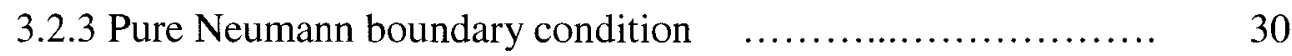

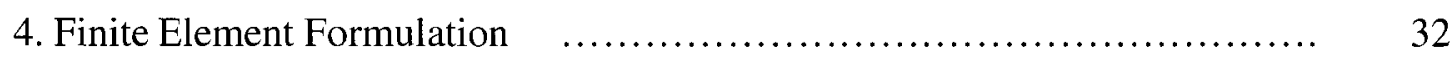

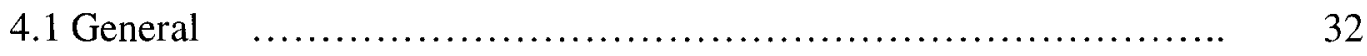

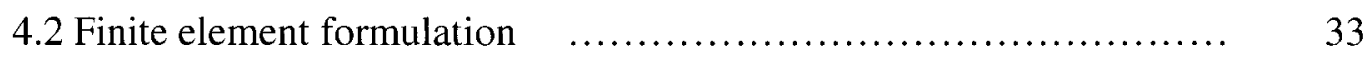

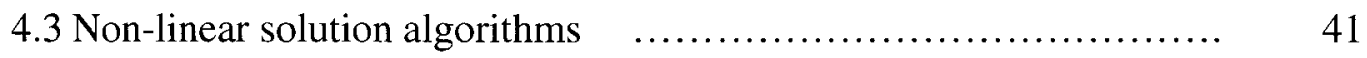

4.3.1 Direction iteration method (Pichard iteration) $\quad \ldots \ldots \ldots \ldots \ldots . . . . .41$

4.3.2 Modified direction iteration method (MDIM) $\quad \ldots \ldots \ldots \ldots \ldots . . . \ldots 46$

4.4 Convergence criterion $\quad \ldots \ldots \ldots \ldots \ldots \ldots \ldots \ldots \ldots \ldots \ldots \ldots \ldots \ldots . \ldots \ldots$

4.5 Developed computer program $\quad$................................ 50

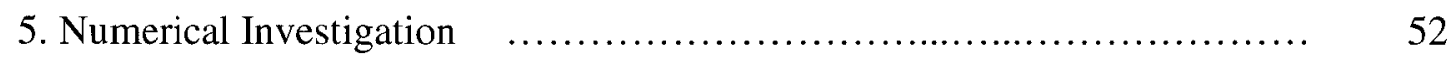

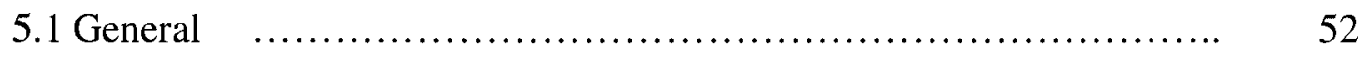

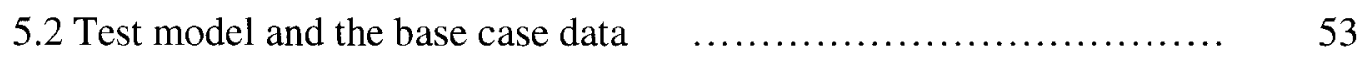

5.3 Solution by direct iteration method (DIM) $\quad \ldots \ldots \ldots \ldots \ldots \ldots \ldots \ldots . \ldots 5$

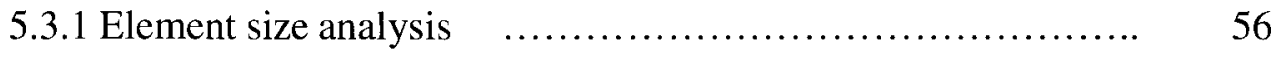

5.3.2 Effect of Anode-to-cathode ratio $\quad \ldots \ldots \ldots \ldots \ldots \ldots \ldots \ldots \ldots . \ldots \ldots$ 
5.3.3 Effect of resistivity

5.4 Solution by modified direct iteration method (MDIM) $\quad \ldots \ldots \ldots \ldots \ldots .63$

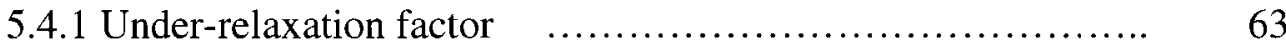

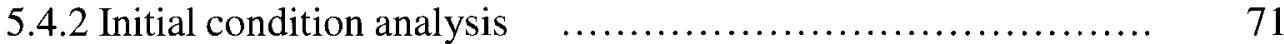

5.4.3 Limiting current density control factor $\quad \ldots \ldots \ldots \ldots \ldots \ldots \ldots . . . \ldots . \ldots . \ldots$

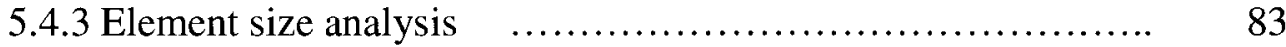

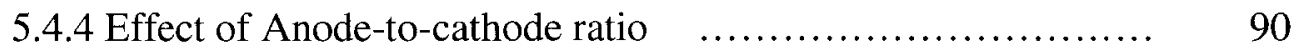

5.4.5 Effect of resistivity $\quad$..................................... 97

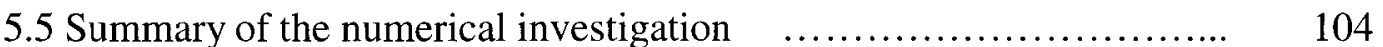

6. The Effect of Corrosion Parameters $\quad$................................... 107

6.1 General $\quad$..................................................... 107

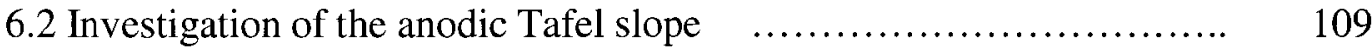

6.3 Investigation of the cathodic Tafel slope $\quad \ldots \ldots \ldots \ldots \ldots \ldots \ldots \ldots \ldots \ldots . . \ldots \ldots$

6.4 Investigation of the anodic exchange current density $\quad \ldots \ldots \ldots \ldots \ldots . . \ldots 119$

6.5 Investigation of the cathodic exchange current density $\quad \ldots \ldots \ldots \ldots \ldots . . . . . \quad 124$

6.6 Investigation of the anodic half cell potential $\quad \ldots \ldots \ldots \ldots \ldots \ldots \ldots \ldots . . . \ldots \ldots$

6.7 Investigation of the cathodic half cell potential $\quad \ldots \ldots \ldots \ldots \ldots \ldots \ldots . . . . . . .6134$

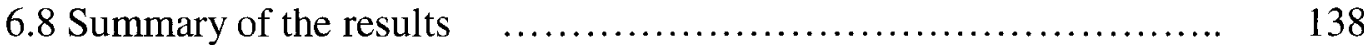

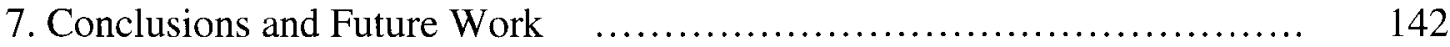

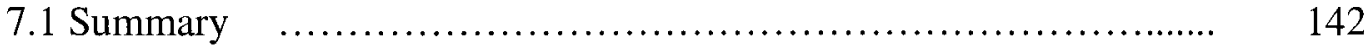

vi 


\section{Acknowledgements}

I would like to thank my supervisor and mentor Prof. O. Burkan Isgor for the guidance and help he provided during this study. His patience, constructive criticism, encouragement and understanding, have helped me overcome the most challenging times that I have encountered during the course of this study. It would be impossible to bring this work to this level without his moral, professional, and financial support. It has been a pleasure to be his student.

I would also like to express my gratitude to my parents for their many years of encouragement.

Finally, I wish to thank my wonderful children, ZeHao and Chelsea, who are the light of my life, the joy of my days, and especially to my wife for her understanding and assistance in my career.

viii 


\section{LIST OF FIGURES}

Fig. 2.1: Influence of w/c ratio, concrete cover, and water saturation on corrosion current density (Source: Balabanic 1996)

Fig. 2.2: Variation of corrosion current density as a function of $\mathrm{pH}$ (Source: Garces et al. 2004)

Fig. 3.1: Corrosion of steel in concrete

Fig. 3.2: Polarization of the steel surface

Fig. 3.3: Boundary conditions defined as Dirichlet type

Fig. 3.4: $\quad$ Boundary conditions defined as mixed Dirichlet/Neumann type $\quad \ldots . . . . \quad 30$

Fig. 4.1: Boundary value problem of a typical corroding system (Source: Isgor 2006)

Fig. 4.2: Boundary conditions on a surface element

Fig. 4.3: Four-noded rectangular element

Fig. 4.4: Flowchart of the DIM

Fig. 4.5: Converged solution using the DIM at a cathodic node

Fig. 4.6: Converged solution using the DIM at an anodic node

Fig. 4.7: Diverged solution by DIM at a cathodic node

Fig. 4.8: The effect of limiting current density on the convergence at a cathodic node

Fig. 4.9: Flowchart of the MDIM 
Fig. 4.10: Solution using the MDIM at two different cathodic nodes

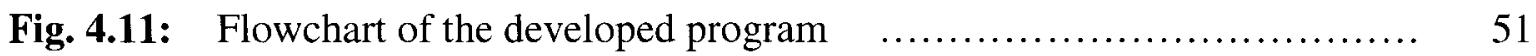

Fig. 5.1: Test model used in the present numerical investigation $\quad \ldots \ldots \ldots \ldots \ldots . . . .64$

Fig. 5.2: Potential distribution calculated using the DIM (Element size analysis) . 57

Fig. 5.3: Current density calculated using the DIM (Element size analysis) $\quad \ldots . . \quad 57$

Fig. 5.4: Potential distribution in the domain calculated using the DIM (Element

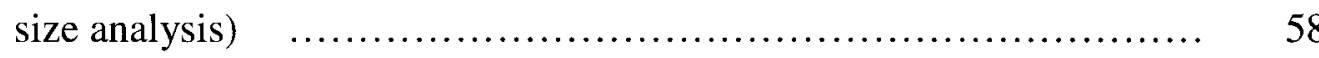

Fig. 5.5: Potential distribution calculated using the DIM (A/C analysis) $\quad \ldots \ldots \ldots \quad 59$

Fig. 5.6: Current density calculated using the DIM (A/C analysis) $\quad \ldots \ldots \ldots \ldots . .60$

Fig. 5.7: Potential distribution in the domain calculated using the DIM $(A / C=$ $0.111)$

Fig. 5.8: Potential distribution calculated using the DIM (Resistivity analysis) .

Fig. 5.9: Current density calculated using the DIM (Resistivity analysis)

Fig. 5.10: Potential distribution in the domain calculated using the DIM ( $r=10,000$ ohm-mm)

Fig. 5.11: Potential distribution on the rebar for different $\xi$ factors $\left(i_{L}=1 \times 10^{-6}\right.$ $\left.\mathrm{A} / \mathrm{mm}^{2}\right)$

Fig. 5.12: Current density on the rebar for different $\xi$ factors $\left(i_{L}=1 \times 10^{-6} \mathrm{~A} / \mathrm{mm}^{2}\right)$

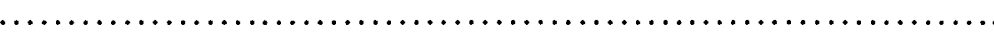

Fig. 5.13: Convergence process for different under-relaxation factors when $i_{L}=$ 
$1 \times 10^{-6} \mathrm{~A} / \mathrm{mm}^{2}$ (a) $\xi=0.003-0.05$ (b) $\xi=0.1-0.5$

Fig. 5.14: Potential distribution on the rebar for different $\xi$ factors $\left(i_{L}=1 \times 10^{-7}\right.$ $\mathrm{A} / \mathrm{mm}^{2}$ ) 68

Fig. 5.15: Current density on the rebar for different $\xi$ factors $\left(i_{L}=1 \times 10^{-7} \mathrm{~A} / \mathrm{mm}^{2}\right)$ (...................................................

Fig. 5.16: Convergence process for different $\xi$ factors $\left(i_{L}=1 \times 10^{-7} \mathrm{~A} / \mathrm{mm}^{2}\right) \quad \ldots$

Fig. 5.17: Potential distribution on the rebar for different $\xi$ factors $\left(i_{L}=1 \times 10^{-8}\right.$ $\mathrm{A} / \mathrm{mm}^{2}$ )

Fig. 5.18: Current density on the rebar for different $\xi$ factors $\left(i_{L}=1 \times 10^{-8} \mathrm{~A} / \mathrm{mm}^{2}\right)$

Fig. 5.19: Convergence process for different under-relaxation factors when $i_{L}=$

$$
1 \times 10^{-8} \mathrm{~A} / \mathrm{mm}^{2} \text { (a) } \xi=0.1-0.5 \text { (b) } \xi=0.003-0.05
$$

Fig. 5.20: Potential distribution on the rebar for different initial conditions $\quad \ldots \ldots . \quad 72$

Fig. 5.21: Current density distribution on the rebar for different initial conditions . 73

Fig. 5.22: Convergence process for different initial conditions $\quad \ldots \ldots \ldots \ldots \ldots \ldots . \ldots 73$

Fig. 5.23: The end part of the cathodic polarization curve $\quad \ldots \ldots \ldots \ldots \ldots \ldots \ldots \ldots \ldots \ldots$

Fig. 5.24: Potential distribution on the rebar for various values of $\psi \quad \ldots \ldots \ldots \ldots . .76$

Fig. 5.25: Current density distribution on the rebar for various values of $\psi \quad \ldots \ldots \quad 76$

Fig. 5.26: Convergence process for various values of $\psi \quad \ldots \ldots \ldots \ldots \ldots \ldots \ldots \ldots \ldots \ldots \ldots$

Fig. 5.27: Potential distribution on the rebar when two $\psi$ values are used $\quad \ldots . . . \quad 79$ 
Fig. 5.28: Potential distribution on the rebar for different values of $i_{L}$

Fig. 5.29: Current density distribution on the rebar for different values of $i_{L} \quad \ldots \ldots \quad 81$

Fig. 5.30: Convergence process for different values of $i_{L}$

Fig. 5.31: Potential and current density distribution in the domain calculated by using the $\operatorname{MDIM}\left(i_{L}=1 \times 10^{-6} \mathrm{~A} / \mathrm{mm}^{2}\right)$

Fig. 5.32: Potential and current density distribution in the domain calculated by using the $\operatorname{MDIM}\left(i_{L}=1 \times 10^{-7} \mathrm{~A} / \mathrm{mm}^{2}\right)$

Fig. 5.33: Potential and current density distribution in the domain calculated by using the $\operatorname{MDIM}\left(i_{L}=1 \times 10^{-8} \mathrm{~A} / \mathrm{mm}^{2}\right)$

Fig. 5.34: Potential distribution on the rebar for $i_{L}=1 \times 10^{-6} \mathrm{~A} / \mathrm{mm}^{2}$ (Element size analysis)

Fig. 5.35: Potential distribution on the rebar for $i_{L}=1 \times 10^{-7} \mathrm{~A} / \mathrm{mm}^{2}$ (Element size analysis)

Fig. 5.36: Potential distribution on the rebar for $i_{L}=1 \times 10^{-8} \mathrm{~A} / \mathrm{mm}^{2}$

Fig. 5.37: Current density distribution on the rebar for $i_{L}=1 \times 10^{-6} \mathrm{~A} / \mathrm{mm}^{2}$ (Element size analysis)

Fig. 5.38: Current density distribution on the rebar for $i_{L}=1 \times 10^{-7} \mathrm{~A} / \mathrm{mm}^{2}$ (Element size analysis)

Fig. 5.39: Current density distribution on the rebar for $i_{L}=1 \times 10^{-8} \mathrm{~A} / \mathrm{mm}^{2}$ (Element size analysis) 
Fig. 5.40: Convergence process for $i_{L}=1 \times 10^{-6} \mathrm{~A} / \mathrm{mm}^{2}$ (Element size analysis) $\quad \ldots \quad 89$

Fig. 5.41: Convergence process for $i_{L}=1 \times 10^{-7} \mathrm{~A} / \mathrm{mm}^{2}$ (Element size analysis) $\quad \ldots \quad 89$

Fig. 5.42: Convergence process for $i_{L}=1 \times 10^{-8} \mathrm{~A} / \mathrm{mm}^{2}$ (Element size analysis) $\quad \ldots \quad 90$

Fig. 5.43: Potential distribution on the rebar for $i_{L}=1 \times 10^{-6} \mathrm{~A} / \mathrm{mm}^{2}$ (A/C analysis) .. $\quad 93$

Fig. 5.44: Potential distribution on the rebar for $i_{L}=1 \times 10^{-7} \mathrm{~A} / \mathrm{mm}^{2}$ (A/C analysis) .. $\quad 93$

Fig. 5.45: Potential distribution on the rebar for $i_{L}=1 \times 10^{-8} \mathrm{~A} / \mathrm{mm}^{2}$ (A/C analysis) .. $\quad 94$

Fig. 5.46: Current density distribution on the rebar for $i_{L}=1 \times 10^{-6} \mathrm{~A} / \mathrm{mm}^{2}(\mathrm{~A} / \mathrm{C}$

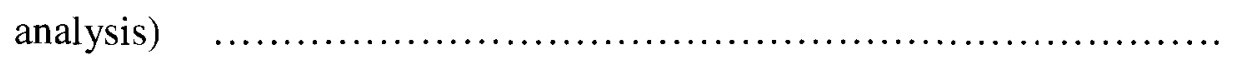

Fig. 5.47: Current density distribution on the rebar for $i_{L}=1 \times 10^{-7} \mathrm{~A} / \mathrm{mm}^{2}(\mathrm{~A} / \mathrm{C}$

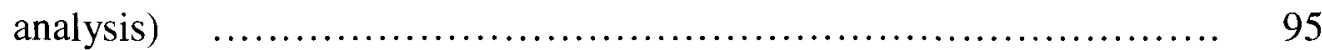

Fig. 5.48: Current density distribution on the rebar for $i_{L}=1 \times 10^{-8} \mathrm{~A} / \mathrm{mm}^{2}(\mathrm{~A} / \mathrm{C}$ analysis)

Fig. 5.49: Convergence process for $i_{L}=1 \times 10^{-6} \mathrm{~A} / \mathrm{mm}^{2}(\mathrm{~A} / \mathrm{C}$ analysis $) \quad \ldots \ldots \ldots \ldots . . .66$

Fig. 5.50: Convergence process for $i_{L}=1 \times 10^{-7} \mathrm{~A} / \mathrm{mm}^{2}$ (A/C analysis) $\quad \ldots \ldots \ldots \ldots . . . .66$

Fig. 5.51: Convergence process for $i_{L}=1 \times 10^{-8} \mathrm{~A} / \mathrm{mm}^{2}$ (A/C analysis) $\quad \ldots \ldots \ldots \ldots . .97$

Fig. 5.52: Potential distribution on the rebar for $i_{L}=1 \times 10^{-6} \mathrm{~A} / \mathrm{mm}^{2}$ (Resistivity analysis)

Fig. 5.53: Potential distribution on the rebar for $i_{L}=1 \times 10^{-7} \mathrm{~A} / \mathrm{mm}^{2}$ (Resistivity analysis)

Fig. 5.54: Potential distribution on the rebar for $i_{L}=1 \times 10^{-8} \mathrm{~A} / \mathrm{mm}^{2}$ (Resistivity 101 
analysis)

Fig. 5.55: Current density distribution on the rebar for $i_{L}=1 \times 10^{-6} \mathrm{~A} / \mathrm{mm}^{2}$ (Resistivity analysis)

Fig. 5.56: Current density distribution on the rebar for $i_{L}=1 \times 10^{-7} \mathrm{~A} / \mathrm{mm}^{2}$ (Resistivity analysis)

Fig. 5.57: Current density distribution on the rebar for $i_{L}=1 \times 10^{-8} \mathrm{~A} / \mathrm{mm}^{2}$ (Resistivity analysis)

Fig. 5.58: Convergence process for $i_{L}=1 \times 10^{-6} \mathrm{~A} / \mathrm{mm}^{2}$ (Resistivity analysis) $\quad \ldots \ldots \quad 103$

Fig. 5.59: Convergence process for $i_{L}=1 \times 10^{-7} \mathrm{~A} / \mathrm{mm}^{2}$ (Resistivity analysis) $\quad \ldots \ldots \quad 103$

Fig. 5.60: Convergence process for $i_{L}=1 \times 10^{-8} \mathrm{~A} / \mathrm{mm}^{2}$ (Resistivity analysis) $\quad \ldots \ldots \quad 104$

Fig. 5.61: Comparison of the DIM and the MDIM for the base case $\left(i_{L}=1 \times 10^{-6}\right.$ $\mathrm{A} / \mathrm{mm}^{2}$ ) (a) Potential distribution (b) Convergence process 106

Fig. 6.1: The effect of anodic Tafel slope on potential distribution $(\mathrm{A} / \mathrm{C}=0.111) \quad 111$

Fig. 6.2: The effect of anodic Tafel slope on current density $(\mathrm{A} / \mathrm{C}=0.111) \quad \ldots \ldots \quad 111$

Fig. 6.3: The effect of anodic Tafel slope on convergence rate $(A / C=0.111) \quad \ldots \quad 112$

Fig. 6.4: The effect of anodic Tafel slope on potential distribution $(\mathrm{A} / \mathrm{C}=1.0) \quad \ldots \quad 112$

Fig. 6.5: The effect of anodic Tafel slope on current density $(\mathrm{A} / \mathrm{C}=1.0) \quad \ldots \ldots \ldots \quad 113$

Fig. 6.6: The effect of anodic Tafel slope on convergence rate $(\mathrm{A} / \mathrm{C}=1.0) \quad \ldots \ldots \quad 113$

Fig. 6.7: The change in corrosion current rate vs. the change in anodic Tafel slope 114

Fig. 6.8: The effect of cathodic Tafel slope on potential distribution $(\mathrm{A} / \mathrm{C}=0.111) \quad 116$ 
Fig. 6.9: The effect of cathodic Tafel slope on current density $(\mathrm{A} / \mathrm{C}=0.111) \quad \ldots \quad 116$

Fig. 6.10: The effect of anodic Tafel slope on convergence rate $(A / C=0.111) \quad \ldots \quad 117$

Fig. 6.11: The effect of cathodic Tafel slope on potential distribution $(\mathrm{A} / \mathrm{C}=1.0) \quad . \quad 117$

Fig. 6.12: The effect of cathodic Tafel slope on current density $(\mathrm{A} / \mathrm{C}=1.0) \quad \ldots \ldots \ldots \quad 118$

Fig. 6.13: The effect of anodic Tafel slope on convergence rate $(\mathrm{A} / \mathrm{C}=1.0) \quad \ldots \ldots \ldots 118$

Fig. 6.14: The change in corrosion current rate vs. the change in cathodic Tafel

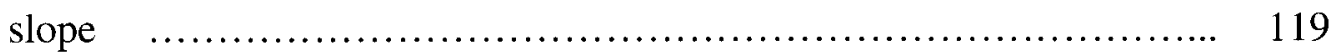

Fig. 6.15: The effect of $i_{o a}$ on potential distribution $(\mathrm{A} / \mathrm{C}=0.111) \quad \ldots \ldots \ldots \ldots \ldots, 121$

Fig. 6.16: The effect of $i_{o a}$ on current density $(\mathrm{A} / \mathrm{C}=0.111) \quad \ldots \ldots \ldots \ldots \ldots \ldots \ldots \ldots \ldots \ldots$

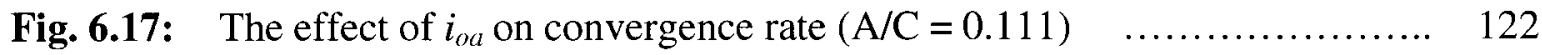

Fig. 6.18: The effect of $i_{o a}$ on potential distribution $(\mathrm{A} / \mathrm{C}=1.0) \quad \ldots \ldots \ldots \ldots \ldots \ldots \ldots \ldots \ldots \ldots$

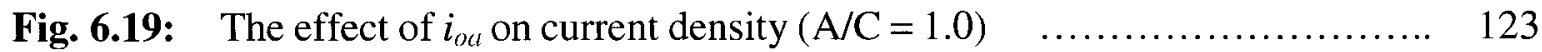

Fig. 6.20: The effect of $i_{o l}$ on convergence rate $(\mathrm{A} / \mathrm{C}=1.0) \quad \ldots \ldots \ldots \ldots \ldots \ldots \ldots \ldots \ldots \ldots$

Fig. 6.21: The change in corrosion current rate vs. the anodic exchange current density

Fig. 6.22: The effect of $i_{o c}$ on potential distribution $(\mathrm{A} / \mathrm{C}=0.111) \quad \ldots \ldots \ldots \ldots \ldots \ldots \ldots$

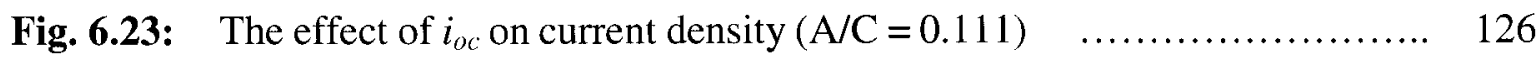

Fig. 6.24: The effect of $i_{o c}$ on convergence rate $(\mathrm{A} / \mathrm{C}=0.111) \quad \ldots \ldots \ldots \ldots \ldots \ldots \ldots \ldots \ldots \ldots$

Fig. 6.25: The effect of $i_{o c}$ on potential distribution $(\mathrm{A} / \mathrm{C}=1.0) \quad \ldots \ldots \ldots \ldots \ldots \ldots . \ldots \ldots$

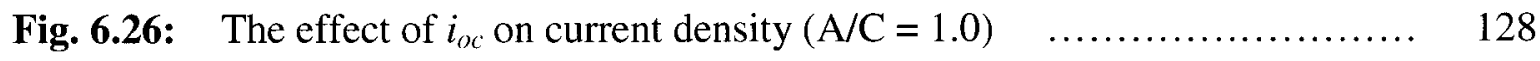


Fig. 6.27: The effect of $i_{o c}$ on convergence rate $(\mathrm{A} / \mathrm{C}=1.0)$

Fig. 6.28: The change in corrosion current rate vs. the cathodic exchange current density

Fig. 6.29: The effect of $E_{o a}$ on potential distribution $(\mathrm{A} / \mathrm{C}=0.111)$

Fig. 6.30: The effect of $E_{o a}$ on current density $(\mathrm{A} / \mathrm{C}=0.111)$

Fig. 6.31: The effect of $E_{o a}$ on convergence rate $(\mathrm{A} / \mathrm{C}=0.111)$

Fig. 6.32: The effect of $E_{o a}$ on potential distribution $(\mathrm{A} / \mathrm{C}=1.0)$

Fig. 6.33: The effect of $E_{o a}$ on corrosion current $(\mathrm{A} / \mathrm{C}=1.0)$

Fig. 6.34: The effect of $E_{o a}$ on convergence rate $(\mathrm{A} / \mathrm{C}=1.0)$

Fig. 6.35: The change in corrosion current rate vs. the anodic half cell potential $\quad \ldots \quad 133$

Fig. 6.36: The effect of $E_{o c}$ on potential distribution $(\mathrm{A} / \mathrm{C}=0.111)$

135

Fig. 6.37: The effect of $E_{o c}$ on current density $(\mathrm{A} / \mathrm{C}=0.111)$

Fig. 6.38: The effect of $E_{o c}$ on convergence rate $(\mathrm{A} / \mathrm{C}=0.111)$

Fig. 6.39: The effect of $E_{o c}$ on potential distribution $(\mathrm{A} / \mathrm{C}=1.0)$

Fig. 6.40: The effect of $E_{o c}$ on current density $(\mathrm{A} / \mathrm{C}=1.0)$

Fig. 6.41: The effect of $E_{o c}$ on convergence rate $(\mathrm{A} / \mathrm{C}=1.0)$

Fig. 6.42: The change in corrosion current rate vs. the cathodic half cell potential . 138

Fig. 6.43: Summary of the effects of corrosion parameters on corrosion rate $(A / C=$ 0.111)

Fig. 6.44: Comparison of the effect of each parameter on corrosion rate using the 
Fig. 6.45: Summary of the effects of corrosion parameters on corrosion rate $(A / C=$

1.0)

Fig. 6.46: Comparison of the effect of each parameters on corrosion rate using the slopes of the linear portion of the change curves $(\mathrm{A} / \mathrm{C}=1.0)$

Fig. A.1: Definition of geometrical properties

Fig. A.2: Definition of environmental parameters

Fig. A.3: Definition corrosion parameters that are used to calculate boundary conditions

Fig. A.4: Activation of boundary condition setup

Fig. A.5: Definition of anodic boundary conditions

Fig. A.6: Definition of cathodic boundary conditions

Fig. A.7: Analysis options

Fig. A.8: Outputting results

Fig. A.9: Saving data files 


\section{LIST OF TABLES}

Table 2.1: Values of corrosion parameters by different researchers

Table 2.2: Tafel slopes for anodic and cathodic reactions at different $\mathrm{pH}$ levels

(Source: Garces et. al. 2005)

Table 5.1: Input parameters of the base case

Table 5.2: $\quad \mathrm{A} / \mathrm{C}$ ratio analysis data and results by the DIM

Table 5.3: Resistivity analysis data and results by the DIM

Table 5.4: Data and results for under-relaxation factor analysis

Table 5.5: Data and results for initial condition analysis

Table 5.6: Data and results of the limiting current density correction factor analysis

Table 5.7: The effect of limiting current density on the solution using MDIM $\quad$.. $\quad 80$

Table 5.8: Element size analysis data and results by the MDIM

Table 5.9: $\quad \mathrm{A} / \mathrm{C}$ ratio analysis data and results by the MDIM

Table 5.10: Resistivity analysis data and results by the MDIM 


\section{Notations}

$$
\begin{aligned}
& \alpha \quad=\text { transfer coefficient } \\
& \alpha_{a} \quad=\text { anodic transfer coefficient } \\
& \alpha_{c} \quad=\text { cathodic transfer coefficient } \\
& \beta_{a} \quad=\text { Tafel slope of the anodic reaction }(\mathrm{mV}) \\
& \beta_{c} \quad=\text { Tafel slope of the cathodic reaction }(\mathrm{mV}) \\
& \lambda=\text { transition ratio } \\
& \Psi \quad=\text { limiting current density control factor } \\
& \Psi(a) \quad=\text { non-linear discrete equation operator } \\
& \delta=\text { thickness of the stagnant layer of electrolyte around the steel surface }(\mathrm{cm}) \\
& \Delta i_{a, a v g}=\text { the change in corrosion rate }\left(\mathrm{A} / \mathrm{mm}^{2}\right) \\
& \phi, \mathrm{E} \quad=\operatorname{potential}(\mathrm{mV}) \\
& \phi_{a} \quad=\text { anodic potentials }(\mathrm{mV}) \\
& \phi_{b}=\text { the Dirichlet type boundary value }(\mathrm{mV}) \\
& \phi_{c} \quad=\text { cathodic potentials }(\mathrm{mV}) \\
& \phi^{\circ}{ }_{F e} \quad=\text { standard half-cell potentials of iron }(\mathrm{mV}) \\
& \phi^{\prime \prime} O_{2} \quad=\text { standard half-cell potentials of oxygen }(\mathrm{mV}) \\
& \Gamma_{a} \quad=\text { anodic boundaries of the domain } \\
& \Gamma_{c} \quad=\text { cathodic boundaries of the domain } \\
& \Gamma_{\text {metal }}=\text { boundary surface between the steel and the concrete interface }
\end{aligned}
$$




$$
\begin{aligned}
& \Gamma_{o} \quad=\text { insulated boundaries of the domain } \\
& \xi \quad=\text { under-relaxation factor } \\
& \eta_{c c} \quad=\text { related to the polarized potential }(\mathrm{mV}) \\
& \theta \quad=\text { angle between the outward normal of the surface and } \mathrm{x} \text {-direction } \\
& \Omega \quad=\text { domain of the boundary value problem } \\
& a \quad=\text { represents nodal value } \\
& {[B] \quad=\text { gradient vector }} \\
& C_{0} \quad=\text { oxygen concentration }\left(\mathrm{kg} / \mathrm{m}^{3}\right) \\
& C_{0}^{s} \quad=\text { dissolved oxygen concentration at the external concrete surface }\left(\mathrm{kg} / \mathrm{m}^{3}\right) \\
& D_{0} \quad=\text { coefficient of oxygen diffusion through concrete }\left(\mathrm{m}^{2} / \mathrm{s}\right) \\
& \mathrm{E}_{\mathrm{corr}} \quad=\text { equilibrium corrosion potential }(\mathrm{mV}) \\
& f\left(i^{k}\right), \eta \quad=\text { polarization equation function } \\
& f \quad=\text { force or source term } \\
& F \quad=\text { Faraday's constant }\left(9.65 \times 10^{4} \mathrm{C} / \mathrm{mol}\right) \\
& i \quad=\text { current density }\left(\mathrm{A} / \mathrm{mm}^{2}\right) \\
& i_{a} \quad=\text { anodic current density }\left(\mathrm{A} / \mathrm{mm}^{2}\right) \\
& i_{c} \quad=\text { cathodic current density }\left(\mathrm{A} / \mathrm{mm}^{2}\right) \\
& i_{L} \quad=\text { limiting current density of the cathodic reaction }\left(\mathrm{A} / \mathrm{mm}^{2}\right) \\
& i_{n} \quad=\text { calculated anodic current density at node } n\left(\mathrm{~A} / \mathrm{mm}^{2}\right) \\
& i_{n}^{b} \quad=\text { anodic current density at node } n \text { for the base case }\left(\mathrm{A} / \mathrm{mm}^{2}\right)
\end{aligned}
$$


$i_{o a} \quad=$ anodic exchange current density $\left(\mathrm{A} / \mathrm{mm}^{2}\right)$

$i_{o c} \quad=$ cathodic exchange current density $\left(\mathrm{A} / \mathrm{mm}^{2}\right)$

$i_{1}, i_{2} \quad=$ current densities calculated by control factors $\Psi_{1}$ and $\Psi_{2}$, respectively $\left(\mathrm{A} / \mathrm{mm}^{2}\right)$

$i_{\text {max,calculated }}=$ largest calculated current density among all nodes along the cathodic region.

$\left(\mathrm{A} / \mathrm{mm}^{2}\right)$

$\{I\} \quad=$ inter-element vector

$\mathrm{I}_{\mathrm{corr}} \quad=$ corrosion current $(\mathrm{A})$

$J_{F} \quad=$ rate of ferrous hydroxide production $\left(\mathrm{kg} / \mathrm{mm}^{2} . \mathrm{s}\right)$

$J_{\text {rust }} \quad=$ related to the current density by Faraday's law $\left(\mathrm{kg} / \mathrm{mm}^{2} . \mathrm{s}\right)$

$K_{L} \quad=$ lower limit of control factors

$K_{U} \quad=$ Upper limit of control factors

$\left[K^{(e)}\right] \quad=$ element stiffness matrix

$\left[k_{b}^{(e)}\right] \quad=$ boundary matrix

$l_{s} \quad=$ distances of the origin of the element from s-axes $(\mathrm{mm})$

$l_{t} \quad=$ distances of the origin of the element from $\mathrm{t}$-axes $(\mathrm{mm})$

$n \quad=$ direction normal to the bar surface

$[N]=$ row vector containing the element shape (interpolation) functions

$N_{a} \quad=$ number of nodes along the anodic surface

$R \quad=$ universal gas constant $(8.3114 \mathrm{~J} / \mathrm{K} . \mathrm{mol})$ 


$\begin{array}{ll}\{R\} & =\text { vector of residuals } \\ \left\{R^{(e)}\right\} & =\text { element (e) matrix of residuals } \\ r & =\text { resistivity of the pore solution }(\Omega-\mathrm{mm}) \\ t_{n} & =\text { transference number of all ions in the solution except for the reduced species } \\ T & =\text { absolute temperature }(\mathrm{K}) \\ {[W]} & =\text { weighting functions of individual elements } \\ z & =\text { number of electrons exchanged in the reaction }\end{array}$




\section{Chapter 1}

\section{Introduction}

\subsection{General}

Corrosion of steel is one of the major causes of deterioration of reinforced concrete structures. Damage induced by corrosion of embedded steel in concrete manifests itself in the form of expansion, cracking, and eventual loss of concrete cover. In extreme cases, the loss of bond between steel and concrete and the reduction of cross sectional area of rebars can cause serious structural damage, even loss of life.

The corrosion of reinforcement in concrete takes place due to a series of electrochemical reactions (Broomfield 1997). Within the high alkaline environment provided by concrete $(\mathrm{pH}>13)$, steel is covered with an insoluble film of ferric oxide (passive layer) which reduces the rates of these electrochemical reactions to insignificant quantities. The partial or complete loss of the passive layer, known as depassivation, may cause the rates of these reactions to increase, leading to the corrosion of steel in concrete. Chloride ion content and $\mathrm{pH}$ of the pore solution around the reinforcement, physical and chemical properties of concrete, surface characteristics and chemical composition of steel, and the presence of mechanical stresses are known to be key factors affecting the depassivation of steel in concrete (Uhlig and Revie 1985). 
It is common practice to idealize the problem of reinforcement corrosion in concrete in two successive stages (Tuutti 1982). The first stage, initiation, can be simply defined as the period during which corrosive agents, such as chloride ions or carbon dioxide, move towards the reinforcement from the surface of concrete, but the steel remains passive. The loss of passivity marks the onset of the second stage, propagation, during which active corrosion of the reinforcement takes place.

The quantification of active corrosion rate of steel in concrete structures through non-destructive and rapid methods is a crucial task for scheduling maintenance/repair operations and for achieving accurate service life predictions. Significant progress has been made on the non-destructive measurement of steel corrosion in concrete since early 1970s, and organizations such as RILEM have already made recommendations for best practices for quantifying corrosion rates (Ghods et al. 2006).

One of the most widely-used methods of measuring corrosion rate in concrete structures is by means of measuring the polarization resistance of the corroding system and using the Stern-Geary equation to calculate the corrosion current density of active steel (Ghods et al. 2006). The calculations that are based on measured polarization resistance data provides instantaneous corrosion rates that are influenced greatly by environmental changes in temperature and humidity; therefore comparisons with average corrosion rates, which can be determined through weight loss measurements, can only be carried out after integrating polarization resistance data over time. From a different perspective, in order to accurately predict the corrosion rates in concrete, one needs to monitor the polarization resistance frequently for a period of time so that temporal variations can be captured. 
Therefore, although the polarization resistance methods are quite practical for instantaneous monitoring of structures, they become significantly expensive and impractical if the monitoring is to be done continuously over long periods.

\subsection{Problem definition}

Accurate modelling of steel corrosion in concrete structures is a very important tool that can help solve the problems associated with using non-destructive testing methods for measuring corrosion rates. Although it is difficult, if not impossible, to completely replace the non-destructive testing methods with numerical models, accurate simulations combined with relatively infrequent, and hence inexpensive, polarization resistance measurements can be the answer to health monitoring of reinforced concrete infrastructure. Although this idea sounds quite promising, numerical modelling of active steel corrosion in concrete has its own challenges that can be summarized under the following categories: (1) estimation of parameters to carry out the simulations; (2) numerical difficulties in the solution of governing equations due to nonlinear boundary conditions; and (3) challenges in modelling complicated geometries (e.g. reinforcement details) and non-homogeneous material properties (Isgor and Razaqpur 2006, Ghods et al. 2006). Despite these challenges, a number of corrosion models that are based on the solution of Laplace's equation for electric potentials have already been developed, as presented in Chapter 2, and some of these models have been verified to be quite effective in simulating well-controlled experimental setups. On the other hand, these numerical models, which are mostly based on direct iteration method, remain to be computationally 
expensive, and in many cases, inefficient as they cannot provide converged solutions to certain problems. In particular, it has been shown that under certain conditions (e.g. small element size, small anode-to-cathode surface are ratio $(\mathrm{A} / \mathrm{C}$ ratio) and extremely low concrete resistivities) the convergence can be very hard to achieve.

\subsection{Objectives and scope of the research}

The following objectives are set for the present study:

- To demonstrate the issues associated with the solution of Laplace's equation with non-linear boundary conditions on steel surface using the direct iteration method.

- To develop a modified version of the direct iteration method to increase the rate of convergence of the solution of Laplace's equation.

- To develop a Windows-based computer program which can be used to carry out a comprehensive study on the solution of Laplace's equation using both approaches.

- To carry out a comprehensive study on how the new method can be most efficiently used under different scenarios.

- To investigate the effect of element size, $\mathrm{A} / \mathrm{C}$ ratio and concrete resistivity on the solution of Laplace's equation by the modified direct iteration method.

- To carry out a parametric study to investigate the effect of variations in corrosion parameters on the rate of steel corrosion in concrete. 
The scope of the research is as follows:

- It is assumed that the reinforcement that is modelled in this research has already been depassivated after an initiation stage. The reason of depassivation (chloride ingress vs. carbonation) is not considered to be a factor in the present study.

- The solution of Laplace's equation is carried out using the finite element method.

- The experimental validation of the accuracy of models that are based on the solution of Laplace's equation for electric potentials to predict the corrosion rates in concrete have already been carried out by previous researchers (Kranc and Sagues 1992, Isgor and Razaqpur 2006). Therefore this study will not focus on the experimental verification, but will only deal with the rate an success of the numerical solution algorithms that are used to solve Laplace's equation.

- There are a number of numerical solution algorithms that can be used to solve non-linear problems (e.g. Newton-Raphson method and Gauss-Seidel iterative technique). However, the nonlinearity in the problem of corrosion of steel in concrete comes from the boundary conditions; therefore methods that are variations of the direct iteration method are generally more appropriate for this problem. Although it is possible to use other solution algorithms, this research focuses only on the direct iteration method and on its variations.

- There are also possible ways of linearizing the boundary conditions (e.g. using Taylor's series expansion) so that convergence can be achieved easily. This study does not use this technique to solve Laplace's equation. 


\subsection{Organization of the thesis}

Chapter 1 provides general introduction to steel corrosion in concrete, problem definition, objectives and scope of this research. Chapter 2 presents the literature review of research related to modelling corrosion in concrete structures. Chapter 3 gives the theoretical background of the corrosion model used in this study. The governing equation and boundary conditions are explained in this chapter. Chapter 4 provides the details of the finite element formulation and nonlinear solution algorithms. The computer program that is developed in this research is also presented in this chapter. Chapter 5 presents the comprehensive numerical study that was carried out to investigate the old and the proposed solution algorithms for the solution of Laplace's equation for steel corrosion under various scenarios. A parametric study to investigate the effect of variations in corrosion parameters on the rate of steel corrosion in concrete is presented in Chapter 6. The main goal of this chapter is to identify the parameters that have significant influence on corrosion rate. Conclusions and recommendations for future work are summarized in Chapter 7. 


\section{Chapter 2}

\section{Literature Review}

\subsection{General}

The mitigation of durability problems related to reinforcement corrosion in concrete involves experimental, analytical and numerical studies of initiation (Papadakis et al. 1991, Saetta et al. 1993, Martin-Perez 1999, Samson et al. 1999, Thomas and Bamforth 1999, Isgor and Razaqpur 2004) and propagation stages (Kranc and Sagues 1992, Feliu 1995, Martin-Perez 1999, Kranc and Sagues 2001, Maruya et al. 2003, Gulikers 2005a,b, Isgor and Razaqpur 2006), as well as of remediation and protection techniques, such as cathodic protection, electrochemical chloride extraction and the use of chemical inhibitors (Broomfield 2000, Hope and Ihekwaba 1995, Fadayomi 1997, Hassanein et al. 1998). These studies, and many more, which are not referenced here, have provided valuable insights into the reinforcement corrosion phenomenon and have contributed to the development of techniques for solving it. In particular, some of the initiation stage models have been demonstrated to yield accurate simulation of the transport of different species in concrete (Papadakis et al. 1991, Saetta et al. 1993, Martin-Perez 1999, Samson et al. 1999, Thomas and Bamforth 1999, Isgor and Razaqpur 2004). On the other hand, despite steady progress, it can be stated that the existing propagation stage models have not advanced to the same level of maturity as the initiation stage models. 
One of the many reasons for slow progress in propagation stage modeling is the difficulties associated with the solution of Laplace's equation with nonlinear boundary conditions. The literature focusing on corrosion (propagation-stage) modelling in concrete structures is quite limited. Among these studies, the ones that address the numerical issues associated with the solution of Laplace's equation do not provide a comprehensive understanding of the problem; rather, they only acknowledge the issue.

It should be noted that many books have been written on the subject of corrosion in general (Uhlig and Revie 1985, Trethewey and Chamberlain 1988, Nestor 2004), and on steel corrosion in concrete in particular (Broomfield 1997, Bentur 1997). Therefore, this review does not go into details of the "vast" corrosion literature; however it focuses on numerical modeling of corrosion in concrete structures. It is acknowledged that the terminology and concepts used in this literature review requires a basic understanding of the corrosion theory. For example, a number of basic concepts such as polarization and Tafel slope are used in this review. Detailed explanation of these basic concepts that are pertinent to corrosion modelling is provided in Chapter 3.

Most numerical models for steel corrosion in concrete are empirical (Yalcin and Ergun 1996, Carino 1999, Mohammed et al. 1999, Jaggi et al. 2001, Wojtas 2003, Garces et al. 2004), or they apply to micro-cell corrosion systems (Gulikers 2005a), in which the anodes and cathodes are not considered to be distinctly identifiable. These models are generally in closed form; hence they do not have any convergence issues associated with the solution of corrosion rates. The research presented in this thesis deals with modelling steel corrosion in concrete in which anodes and cathodes are assumed to form on different 
locations on steel surface and the geometry of the electrochemical system does not allow closed-form solutions.

\subsection{Modelling steel corrosion in concrete}

A number of studies exist to model reinforcement corrosion in concrete as an electrochemical reaction (Harker et al. 1987, Walton and Sagar 1987, Walton et al. 1990, Krank and Sagues 1992, Balabanic et al. 1996, Matsushima et al. 1996, Yokozeki et al. 1997, Martin-Perez 1999, Isgor 2001). Among these studies, the ones conducted by Kranc and Sagues (1992), Balabanic et al. (1996) and Isgor (2001) are important since they use numerical methods to solve the governing equation for reinforcement corrosion in concrete in two-dimensional space, and they are more comprehensive in the fundamentals.

Kranc and Sagues (1992) modeled the case of a reinforcing steel bar experiencing localized corrosion in a concrete cylinder to obtain current distribution. The solution is based on finite difference analysis of Laplace's equation with simplified boundary conditions. In this study, researchers assumed that the anodic surface of steel does not polarize, and the corrosion is only cathodically controlled by the diffusion of oxygen through the concrete cover. This allowed the investigators to model the anodic boundary conditions with a constant potential (Dirichlet condition) so that numerical difficulties associated with the solution of Laplace's equation can be partially avoided. The cathodic boundary conditions and insulated surfaces were modelled as Neumann condition. It is 
well documented (Elsener 2005) that anodic surfaces of steel in concrete polarize, mostly in the form of activation polarization; therefore, the assumption of non-polarizable anodic surfaces can be considered as over-simplification of the problem. However, despite the simplifying assumptions, the convergence of the problem presented in Kranc and Sagues (1992) was quite time consuming, mostly reached after 100,000 iterations. The same researchers have improved their model in the following years, expanding it to more complicated two-dimensional geometries (Kranc and Sagues 2001); however, numerical challenges associated with the solution of Laplace's equation remained. Kranc and Sagues did not carry out a comprehensive study on the numerical difficulties associated with the solution of corrosion rates.

Balabanic et al. (1996) proposed a numerical model for the chloride-induced corrosion of reinforcing steel in concrete. The model also included the numerical solution of Laplace's equation, this time using finite element analysis. In this study, in addition to the solution of Laplace's equation for electric potentials, the researchers also used a finite difference technique to solve the oxygen diffusion problem in concrete to accurately model the cathodic boundary conditions, which are significantly affected by the amount of dissolved oxygen that is present around the cathodic surfaces of the reinforcement. In solving the oxygen diffusion problem, the researchers considered the reaction rates on the steel surface so that the boundary conditions for the oxygen diffusion problem are simulated accurately. The cathodic boundary condition, $\phi_{c}$, in this study was modelled in Dirichlet form as:

Cathode: $\phi_{c}=-0.512-0.0148 \log \left(C_{0} / C_{w}\right)$ 
where $C_{0}$ and $C_{w}$ are the concentrations of oxygen and capillary water around the cathodic surfaces of steel. Similar to Kranc and Sagues study, Balabanic et al. (1996) did not carry out a comprehensive investigation on the numerical difficulties associated with the solution of corrosion rates in concrete.

The numerical results of Balabanic's model signify that the corrosion rate is affected by water-cement ratio, concrete cover thickness and water saturation, as illustrated in Fig. 2.1. In addition, it is well documented that the effect of other parameters such as moisture content, temperature, concrete resistivity and $\mathrm{pH}$ of the pore solution play important roles in corrosion (Uhlig and Revie 1985, Broomfield 1997). For example, Yalcin and Ergun (1996) have experimentally shown that Tafel slope has a significant influence on steel corrosion rate in concrete; and Garces et al. (2004) indicted that $\mathrm{pH}$ level has a impact on the corrosion current as illustrated in Fig. 2.2.

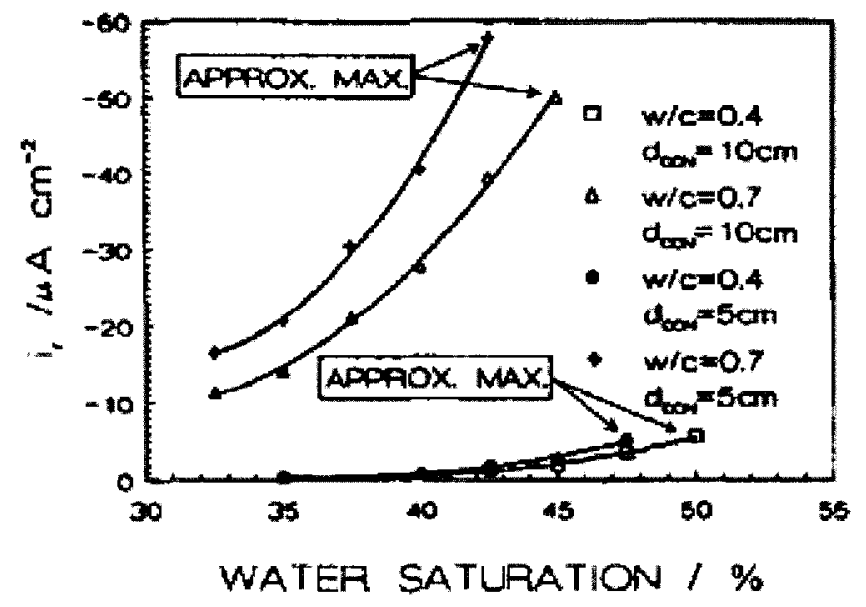

Fig. 2.1: Influence of $w / c$ ratio, concrete cover, and water saturation on corrosion current density (Source: Balabanic 1996) 


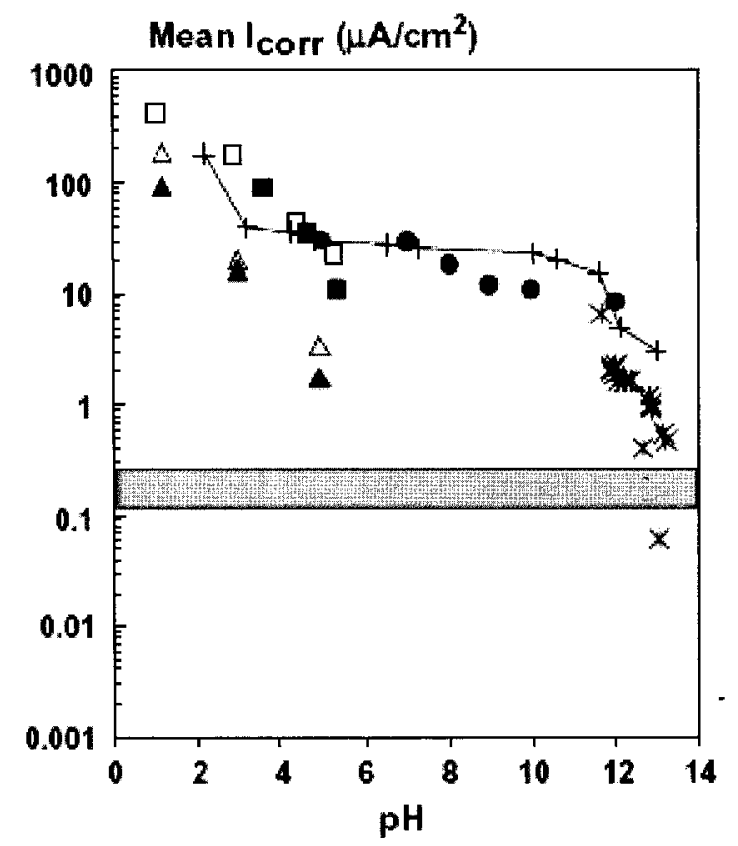

Fig. 2.2: Variation of corrosion current density as a function of $\mathrm{pH}$ (Source: Garces et al. 2004)

In order to develop a more comprehensive approach to corrosion modelling in concrete, Isgor (2001) developed a systematic and robust method for predicting chloride and carbonation-induced steel corrosion in reinforced concrete structures under prescribed exposure conditions (Isgor and Razaqpur 2006). The model consists of initiation and propagation stages which are cast in the same time and space domains; i.e., processes which commence in the initiation stage, such as temperature, moisture, chloride ion, and oxygen transport within concrete, continue in the propagation stage while active corrosion occurs contemporaneously. This allows the model to include the effects of changes in exposure conditions during the propagation stage on corrosion and the effects of the corrosion reactions on the properties of concrete. In this model, the significant phenomena that contribute to the rate and amount of corrosion of reinforcement; i.e. heat and moisture transfer, chloride, carbon dioxide and oxygen transport, are modeled by the two-dimensional time-dependent nonlinear finite element method. The governing 
equations of the various transport phenomena are solved numerically in the time and the space domains. Where appropriate, the coupling effects among the different transport phenomena and the chloride binding/release mechanisms as well as the carbonation process are modeled in order to simulate the field conditions reasonably close. The proposed finite element model and its associated computer program, Condur, are capable of handling any geometry (including discrete cracks), prescribed initial and boundary conditions and pertinent material nonlinearities.

In Isgor's model, the corrosion rates on the steel surface are also calculated by solving the Laplace's equation for electrochemical potential with appropriate boundary conditions. These boundary conditions include the relationship between overpotential and current density for the anodic and cathodic regions. It was assumed that anodes mostly polarize due to activation polarization, and cathodes can polarize through both activation and concentration polarization. Due to the non-linear nature of these boundary conditions, a non-linear solution algorithm, which was based on the direct iteration method (Pichard iteration), was used. To verify the finite element model and its associated computer program, the numerical results are compared with available experimental data, with reasonable agreement between them (Isgor and Razaqpur 2006). However, it was observed that the numerical solution algorithm used in this model had challenges in providing fast convergence. In general, an excess of 10,000 iterations were necessary to reach a convergence of $0.1 \mathrm{mV}$ between two successive iterations at all nodes. Although relaxation algorithms have resulted in faster convergence, convergence was not possible in many cases with low concrete resistivity or low anode-to-cathode ratio. Following this study, Ghods at al. (2006) showed that concrete resistivity, anode-to-cathode ratio and 
oxygen concentration have significant influence on the rate and success of convergence of the solution of Laplace's equation for corrosion rates in concrete.

The solution of Laplace's equation with nonlinear boundary conditions requires that the values of a number of parameters be predetermined. These parameters, which originate from the basic corrosion theory, can be listed as follows:

- Anodic Tafel slope, $\beta_{a}$

- Cathodic Tafel slope, $\beta_{c}$

- Anodic exchange current density, $i_{o x}$

- Cathodic exchange current density, $i_{o c}$

- Standard electrode potential for the anodic reaction, $E_{o u}$

- Standard electrode potential for the cathodic reaction, $E_{o c}$

It should be acknowledged that due to the nature of the corrosion problem in concrete, there is significant uncertainty associated with the selection of these parameters. The literature review conducted on the selection of these parameters confirmed this uncertainty by revealing that different researchers have selected parameters from wide range of possible values as illustrated in Table 2.1.

Experimental evidence also proves that these parameters vary significantly; therefore parameter selection process for modelling it is quite challenging. Garces et al. (2005) studied and measured the corrosion rate of steel bars at different $\mathrm{pH}$ levels in solutions 
simulating chloride environments in the micropores of concrete. The Tafel slopes obtained ion this research revealed that there is a significant effect of $\mathrm{pH}$ as shown in Table 2.2. In other studies (see Elsener, 2005), it was also reported that the anodic Tafel slope can vary from 73 to $98 \mathrm{mV}$ in solution experiments with neutral to acidic $\mathrm{pH}$. In the same studies, it was shown that cathodic Tafel slope can vary from 200 to $230 \mathrm{mV}$.

Table 2.1: Values of corrosion parameters by different researchers

\begin{tabular}{|c|c|c|c|c|}
\hline Parameter & $\begin{array}{c}\text { Kranc and } \\
\text { Sagues (1992) }\end{array}$ & $\begin{array}{c}\text { Kranc and } \\
\text { Sagues (2001) }\end{array}$ & $\begin{array}{c}\text { Isgor and } \\
\text { Razaqpur (2006) }\end{array}$ & $\begin{array}{c}\text { Gulikers } \\
(\mathbf{2 0 0 5 b})\end{array}$ \\
\hline$\beta_{a}(\mathrm{mV})$ & 60 & 60 & Calculated & 91 \\
\hline$\beta_{c}(\mathrm{mV})$ & 160 & 160 & Calculated & 176 \\
\hline$i_{o a}\left(\mathrm{~A} / \mathrm{mm}^{2}\right)$ & $\mathrm{N} / \mathrm{A}$ & $1.875 \times 10^{-10}$ & $2.75 \times 10^{-10}$ & $1.0 \times 10^{-12}$ \\
\hline$i_{o c}\left(\mathrm{~A} / \mathrm{mm}^{2}\right)$ & $2 \times 10^{-10}$ & $6.25 \times 10^{-12}$ & $6 \times 10^{-12}$ & $1.0 \times 10^{-13}$ \\
\hline$E_{o c}(\mathrm{mV}$ vs. SCE $)$ & -600 & -780 & -780 & -689 \\
\hline$E_{o c}(\mathrm{mV}$ vs. SCE) & 0 & 160 & 160 & 159 \\
\hline
\end{tabular}

Table 2.2: Tafel slopes for anodic and cathodic reactions at different $\mathrm{pH}$ levels (Source: Garces et. al. 2005)

\begin{tabular}{|c|c|c|}
\hline Solution No. & $\beta_{a}(\mathbf{m V})$ & $\beta_{c}(\mathbf{m V})$ \\
\hline $1(p H=6.32)$ & 73 & 242 \\
\hline $2(p H=5.30)$ & 136 & 208 \\
\hline $3(p H=4.41)$ & 77 & 123 \\
\hline $4 p H=2.81)$ & 83 & 112 \\
\hline
\end{tabular}




\subsection{Summary of the literature review}

The following conclusions can be drawn from the literature review presented here:

- Corrosion of steel in concrete has been modeled by various researchers in the past; however, most of the existing models are empirical and have limited applicability.

- The models that are based on the solution of Laplace's equation with distinct anodic and cathodic boundary conditions have a wider range of applicability. These models have been shown to provide reasonable predictions for corrosion rates in concrete, as verified by experimental studies.

- The models that are based on the solution of Laplace's equation have problems with convergence. In most cases, the convergence can only be achieved after a large number of iterations. Even after relaxation algorithms are used, the convergence may not be achieved at certain conditions (e.g. low A/C ratio).

- Concrete resistivity, $\mathrm{A} / \mathrm{C}$ ratio and oxygen concentration have been shown to have significant influence on the rate and success of convergence of the solution of Laplace's equation for corrosion rates in concrete. No comprehensive study on the investigation of these parameters on the rate and success of steel corrosion in concrete exists.

- Due to the nature of the corrosion problem in concrete, there is significant uncertainty associated with the selection of corrosion parameters. No comprehensive study on the investigation of the sensitivity of the rate of steel corrosion by these parameters exists. 


\section{Chapter 3}

\section{Modelling Steel Corrosion in Concrete}

\subsection{Corrosion of steel in concrete}

\subsubsection{Electrochemical process}

The corrosion of steel in concrete is a result of the dissolution of iron in pore water which can be represented by the half-cell reactions given in Eqs. 3.1 and 3.2:

$F e \rightarrow F e^{2+}+2 e^{-}$

The electrons that are produced in this anodic reaction must be consumed at the cathodic sites on the steel surface to preserve electrical neutrality. The cathodic reaction in which these free electrons are consumed is the oxygen reduction given by:

$\frac{1}{4} \mathrm{O}_{2}+\frac{1}{2} \mathrm{H}_{2} \mathrm{O}+e^{-} \rightarrow \mathrm{OH}^{-}$

If the anodic reaction given in Eq. 3.1 were the only reaction that took place during the corrosion process, there would be no cracking and spalling of the concrete surrounding steel. However, $\mathrm{Fe}^{2+}$ ions continue to react within the pore solution to form rust. The following reactions represent the formation of the so-called "red rust" after the iron dissolution occurs at the anodic sites on the reinforcement (Broomfield 1997):

$$
\begin{aligned}
& \mathrm{Fe}^{2+}+2 \mathrm{OH}^{-} \rightarrow \mathrm{Fe}(\mathrm{OH})_{2} \\
& 4 \mathrm{Fe}(\mathrm{OH})_{2}+2 \mathrm{H}_{2} \mathrm{O}+\mathrm{O}_{2} \rightarrow 4 \mathrm{Fe}(\mathrm{OH})_{3} \\
& 2 \mathrm{Fe}(\mathrm{OH})_{3} \rightarrow 2 \mathrm{H}_{2} \mathrm{O}+\mathrm{Fe}_{2} \mathrm{O}_{3} \cdot \mathrm{H}_{2} \mathrm{O}
\end{aligned}
$$


$\mathrm{Fe}_{2} \mathrm{O}_{3}$, or red rust, is the corrosion product whose volume is approximately four times as large as the volume of the steel with the same mass. Therefore, the formation of red rust may cause internal stresses and consequently the cracking and spalling of the concrete surrounding the reinforcement. Although the additional reactions other than the ones given in Eqs. 3.1 to 3.5 may take place during the course of the corrosion process, (e.g. hydrogen evolution due to water decomposition at extremely active locations on the steel surface, or the formation of black rust), in the present study, the corrosion of steel in concrete will be defined by the reactions given in Eqs. 3.1-3.5, and as illustrated in Fig. 3.1.

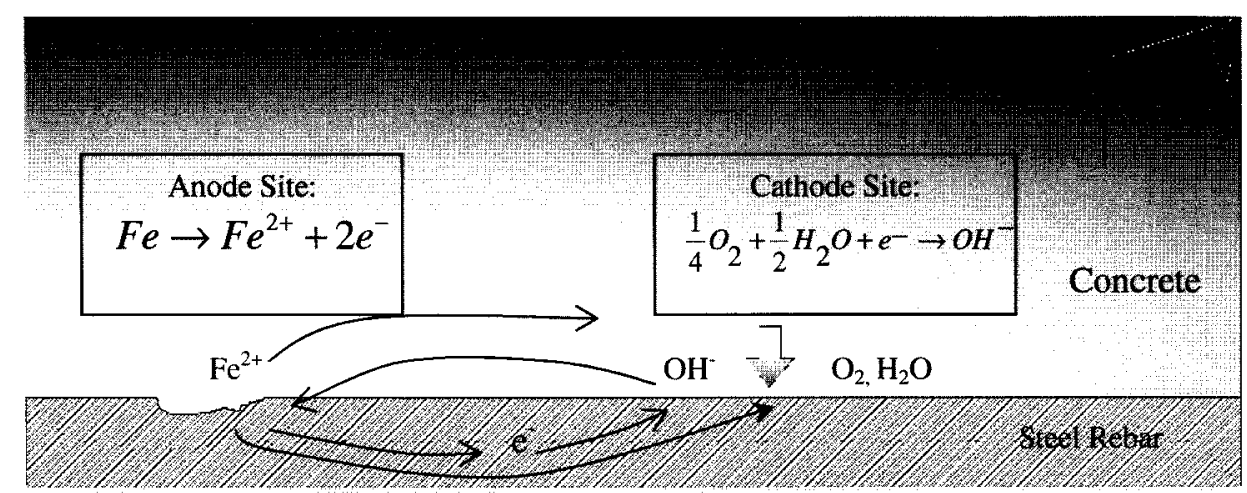

Concrete

Fig. 3.1: Corrosion of steel in concrete

The corrosion rate at any point on the surface of steel in concrete is related to the current density, which can be predicted if the electrochemical potential (abbreviated henceforth as "potential") distribution around that point is known. Once the potential distribution along the reinforcement is known, the current density at any point on the steel surface can be calculated by: 
$i=-\frac{1}{r} \frac{\partial \phi}{\partial n}$

where $i\left[\mathrm{~A} / \mathrm{mm}^{2}\right]$ is the current density, $\phi$ [volts] is the potential, $r[\Omega-\mathrm{mm}$ (ohm-mm)] is the resistivity of the pore solution and $n$ is the direction normal to the bar surface. The rate of rust production at the anodic regions on the steel surface can be calculated by Faraday's law.

The rate of rust production at the anodic regions on the steel surface, $J_{\text {rust }}\left[\mathrm{kg} / \mathrm{mm}^{2} . \mathrm{s}\right]$, is related to the current density by Faraday's law. The rate of ferrous hydoxide, $\mathrm{Fe}(\mathrm{OH})_{2}$, production, $J_{F}$, at the anodic regions according to Eq. 3.3 can be written as:

$$
J_{F}=89.845 \times 10^{-3} \frac{i_{a}}{z F}=4.656 \times 10^{-7} i_{a}
$$

where $i_{a}$ is the anodic current density, $F$ is the Faraday's constant $\left(9.65 \times 10^{4} \mathrm{C} / \mathrm{mol}\right)$, and $z$ is the number of electrons exchanged in the reaction $(z=2) . \mathrm{Fe}(\mathrm{OH})_{2}$ can be further oxidized based on Eq. 3.4, and this will result in the production of $\mathrm{Fe}(\mathrm{OH})_{3}$. Since one mole of $\mathrm{Fe}(\mathrm{OH})_{2}$, which is $89.845 \mathrm{~g}$, produces one mole of $\mathrm{Fe}(\mathrm{OH})_{3}(106.845 \mathrm{~g})$, the rate of rust production, $J_{\text {rust }}$, at the anodic regions on the steel surface can be calculated by:

$$
J_{\text {rust }}=\frac{106.845}{89.845} J_{F}=5.536 \times 10^{-7} i_{a}
$$

Therefore, once the current densities on the steel surface are calculated, the determination of the rate and amount of corrosion becomes a straightforward task. The main difficulty in this process is the calculation of current densities on the steel surface. As it can be observed from Eq. 3.6, the calculation of current densities requires knowledge of the potential distribution around the reinforcement. 


\subsubsection{Governing equation}

The equation governing the potential distribution within an electrochemical system can be derived from first principles (Munn 1982). Assuming electrical charge conservation and isotropic conductivity, the potential distribution in two dimensions can be represented by:

$\frac{\partial}{\partial x}\left(\frac{1}{r_{x}} \frac{\partial \phi}{\partial x}\right)+\frac{\partial}{\partial y}\left(\frac{1}{r_{y}} \frac{\partial \phi}{\partial y}\right)=0$

where $x$ and $y$ are planar Cartesian coordinates, and $r_{x}$ and $r_{y}$ are the resistivities of the pore solution in $\mathrm{x}$ - and $\mathrm{y}$-directions, respectively.

Calculation of the potential distribution around the surface of the steel involves the solution of Eq. 3.9 subject to prescribed boundary conditions. These boundary conditions comprise the relationship between potential and current density for the anodic and cathodic regions as well as prescribed current densities. For the anodic and cathodic regions of the steel surface, the boundary conditions, in Dirichlet form, can be defined as:

$\phi=\phi_{a}$

$\phi=\phi_{c}$

where $\phi_{a}$ and $\phi_{c}$ are the polarized anodic and cathodic potentials, which will be discussed later.

At equilibrium, the rate of the forward and reverse reactions given by Eqs. 3.1 and 3.2 are equal to each other, and the rate of anodic (Eq. 3.1) and cathodic (Eq. 3.2) reactions can be represented by exchange current density $i_{o a}$ and $i_{o c}$, respectively. When the equilibrium is disturbed, a net current starts flowing between the anodic and cathodic sites on the steel, 
and the equilibrium potentials are altered, the extent of which depends on the magnitude of the net current and its direction. The potential change, measured in volts, caused by the net current is called polarization. The basis of the polarization theory is well established (Stern and Geary 1957); however a brief explanation of the phenomenon will be given here.

\subsubsection{Polarization of the steel surface}

Non-standard half-cell potentials of the reactions given in Eqs. 3.1 and 3.2, $\phi_{\mathrm{Fe}}$ and $\phi_{\mathrm{O}_{2}}$ can respectively be represented by the Nernst equation (Uhlig amd Revie 1985):

$$
\begin{gathered}
\phi_{F e}=\phi_{F e}^{o}-\frac{2.303 R T}{2 F} \log \left(\left[F e^{2+}\right]\right) \\
\phi_{O_{2}}=\phi_{O_{2}}^{o}-\frac{2.303 R T}{F} \log \left(\frac{\left[O H^{-}\right]}{P_{O_{2}}^{\frac{1}{4}}}\right)
\end{gathered}
$$

where $\phi^{a} \mathrm{Fe}_{e}$ and $\phi^{o} \mathrm{O}_{2}$ are the standard half-cell potentials of $\mathrm{Fe}$ and $\mathrm{O}_{2}$, respectively, $R$ is the universal gas constant $(8.3114 \mathrm{~J} / \mathrm{K} . \mathrm{mol}), T$ is the absolute temperature, $\left[\mathrm{Fe}^{2+}\right]$ and $\left[\mathrm{OH}^{-}\right]$are the activities of the iron and hydroxyl ions, respectively, and $P_{O 2}$ is the fugacity of $\mathrm{O}_{2}$, which can be approximated as partial pressure of oxygen (Uhlig and Revie 1985).

The electrode potentials in Eqs.3.12 and 3.13 are shifted by polarization. There are three major sources of polarization for steel in concrete: concentration, activation, and resistance. Concentration polarization, also called "diffusion overpotential" or "mass-transport overpotential", is the overpotential associated with the diffusional transport of the reactants to the electrode surface from the bulk of the electrolyte and the 
reverse transport of the products. In the context of steel corrosion in concrete, concentration polarization manifests itself when the amount of dissolved oxygen around cathodic surface is depleted due to the consumption in the reduction reaction given in Eq. 3.2. In general, oxygen diffusion is relatively fast in concrete under aerated conditions; however, there are cases in which diffusion of oxygen through concrete cover is slow, as in the case of submerged structures or in members with high performance concrete.

Activation polarization is the overpotential associated with the charge-transfer reaction elementary step in the overall electrode reaction. Its name comes from the activation energy barrier that is required to be overcome in order for the electrode reactions to continue. Activation polarization in concrete affects both anodic and cathodic reactions; therefore it should be included in the modelling of steel corrosion.

Solutions of electrolytes generally have a rather poor conductivity compared to metals, particularly for dilute solutions. If the solution has a high electrical resistance this will give rise to a potential difference between the anodic and cathodic sites, which is known as resistance polarization. If resistance polarization dominates a reaction, it is referred as resistance or IR controlled (the latter term comes from Ohm's Law).

The corrosion of steel in partially saturated concrete can be considered to be a cathodically controlled process (Broomfield 1997), during which greater polarization occurs at the cathodic sites. Although some researchers have made the assumption that anodes on the steel surface do not polarize during the corrosion process, this may be an oversimplification of the problem. Therefore, in the present work, we will assume that 
anodic sites on the surface of the steel polarize, but mostly in the form of activation polarization. The resistance polarization is generally small, especially when the anodic and cathodic sites are very close to each other. In cases where anodic and cathodic sites are well separated, and the conductivity of the electrolyte (pore solution) is low, as in the case of dry concrete, resistance of the pore solution may also play an important role in the kinetics of the corrosion process. The following discussion provides the details of the polarization process in concrete and the manner in which it is treated in the present model.

The cathodic portions of the steel surface are predominantly polarized by concentration polarization (Uhlig amd Revie 1985). Assuming that the rate of the forward reaction in Eq. 3.2 is significantly larger than the reverse reaction, concentration polarization, $\eta_{c c}$, can be calculated by (Glasstone 1942):

$\eta_{c c}=\frac{2.303 R T}{z F} \log \frac{i_{L}}{i_{L}-i_{c}}$

where $i_{L}\left[\mathrm{~A} / \mathrm{mm}^{2}\right]$ is the limiting current density of the cathodic reaction and $\mathrm{z}=1$. As it can be seen from Eq. 3.14, cathodic current density, $i_{c}$, is related to the polarized potential, $\eta_{c c}$. The limiting current density, $i_{L}$, of the oxygen reduction at the cathodic sites can be determined using (Uhlig amd Revie 1985):

$i_{L}=\frac{3.2 D_{O_{2}} z F}{100 \delta t_{n}} C_{O_{2}}$

where $D_{O 2}\left[\mathrm{~m}^{2} / \mathrm{s}\right]$ is the effective oxygen diffusion coefficient in concrete, $\delta[\mathrm{cm}]$ is the thickness of the stagnant layer of electrolyte around the steel surface, $t_{n}$, is the transference number of all ions in the solution except for the reduced species, and $C_{O 2}$ 
$\left[\mathrm{kg} / \mathrm{m}^{3}\right]$ of pore solution] is the concentration of oxygen around the steel in moles per litre. In the current study, the thickness of the stagnant layer of electrolyte around the steel surface, $\delta$, and the transference number, $t_{n}$, are considered to be input values. However, for simplicity, $\delta$ and $t_{n}$ can be respectively assumed to be equal to $0.05 \mathrm{~cm}$ (since pore solution is an unstirred solution) and 1 (since there are many ions in concrete pore solution) (Uhlig and Revie 1985).

If we assume that the rate of the forward reaction in Eq. 3.2 is significantly larger than the reverse reaction, the activation polarization of the cathodes can be determined from Butler-Volmer kinetics (Bockris and Reddy 1977, Perez 2004) and is represented by the cathodic Tafel equation:

$\eta_{c a}=\beta_{c} \log \frac{i_{c}}{i_{o c}}$

where $\beta_{c}$ is the Tafel slope of the cathodic reaction which is equal to:

$\beta_{c}=\frac{2.303 R T}{\left(1-\alpha_{c}\right) z F}$

in which $\alpha_{c}$ is a cathodic charge transfer coefficient, and $i_{o c}$ is the exchange current density of the cathodic reaction, both being input parameters. Adding all the cathodic polarization components as given by Eqs.3.14 and 3.16, the polarized potential of the cathodic reaction, $\phi_{c}$, can be calculated as:

$$
\phi_{c}=\phi_{O_{2}}+\beta_{c} \log \frac{i_{c}}{i_{o c}}-\frac{2.303 R T}{z F} \log \frac{i_{L}}{i_{L}-i_{c}}
$$

The polarization characteristics of the anodic reaction can be considered to be activation 
limited. Incorporating the resistance polarization for the cases where the pore solution has high resistivity and the anodic and cathodic sites are separated sufficiently, the polarized potential of the anodic reaction can be written as:

$\phi_{a}=\phi_{F e}+\beta_{a} \log \frac{i_{a}}{i_{\text {oa }}}$

where $\beta_{a}$ is the Tafel slope of the anodic reaction which is equal to:

$\beta_{a}=\frac{2.303 R T}{\alpha_{a} z F}$

where $\alpha_{a}$ is anodic charge transfer coefficient.

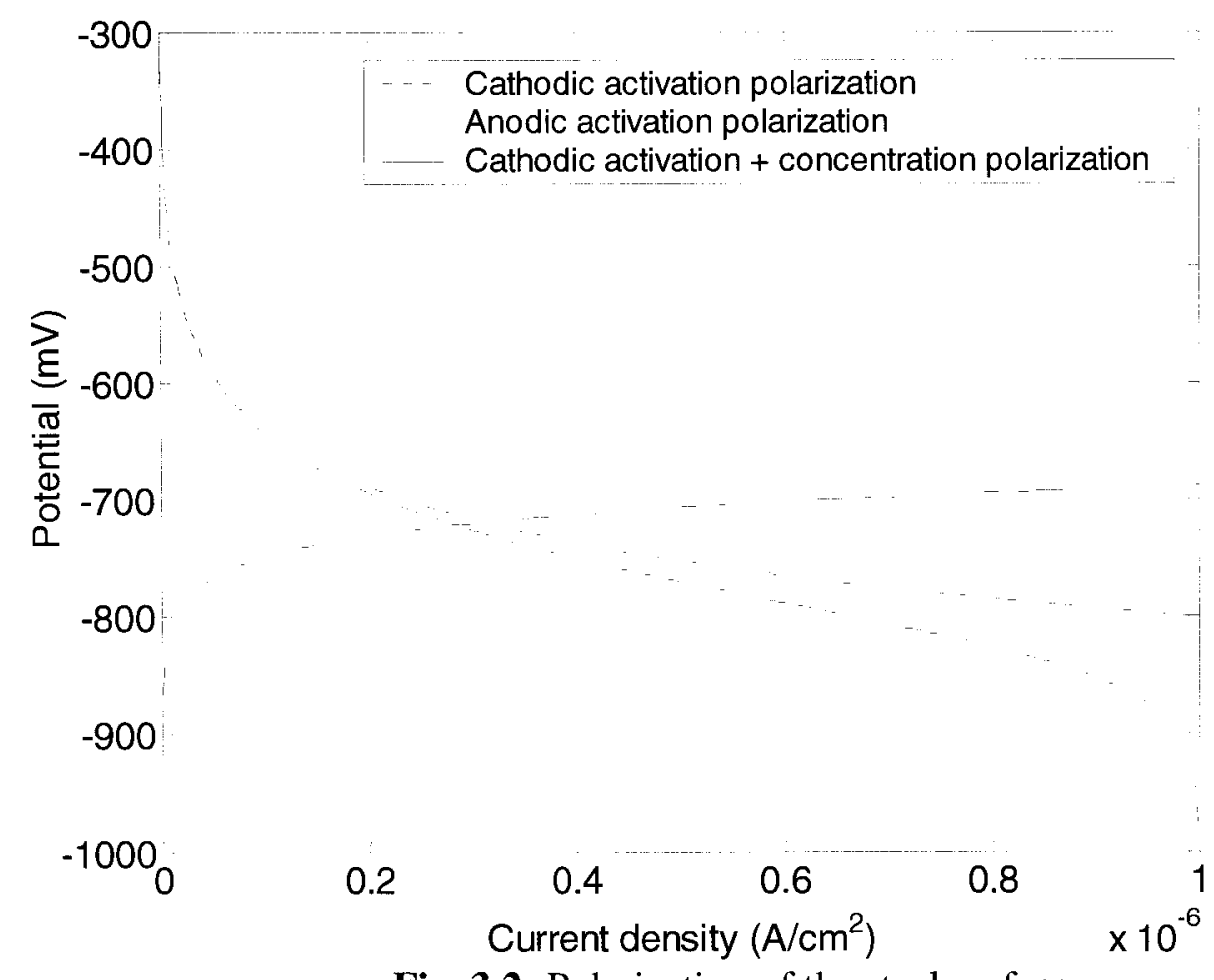

Fig. 3.2: Polarization of the steel surface 


\subsection{Modelling corrosion of steel in concrete}

The typical boundary value problem which governs the corrosion of steel in concrete can be modeled with a simple geometry as shown in Fig. 3.3. The domain and the boundary conditions shown in this figure can be used to model macro-cell pitting corrosion on a reinforcing bar caused by chloride ions, or micro-cell corrosion caused by carbonation.

If the domain is assumed to be homogeneous, i.e. resistivity of the medium is assumed to be constant within the domain, Eq. 3.9 can be written as Laplace's equation

$$
\frac{\partial^{2} \phi}{\partial x^{2}}+\frac{\partial^{2} \phi}{\partial y^{2}}=0 \quad \phi \in \Omega
$$

which can be represented in short form as:

$\nabla^{2} \phi=0$ in $\Omega$

where $\nabla^{2}$ is the usual harmonic operator and $\Omega$ represents the domain of the boundary value problem. Once the potential distribution in the domain is determined after the solution of Eq. 3.22, the current density, $i\left[\mathrm{~A} / \mathrm{cm}^{2}\right]$, on the steel surface can be calculated as:

$$
i=-\frac{1}{r} \frac{\partial \phi}{\partial n} \quad \phi \subset \Gamma_{\text {metal }}
$$

where $r[\Omega-\mathrm{cm}(\mathrm{ohm}-\mathrm{cm})]$ is the resistivity of the pore solution, $n$ is the direction perpendicular to the equipotential lines, and $\Gamma_{\text {metal }}$ represents the boundary surface between the steel and the concrete interface. The gradient term in Eq. 3.23 can also be written in two dimensional Cartesian form as: 
$\frac{\partial \phi}{\partial n}=\left\{\begin{array}{l}\frac{\partial \phi}{\partial x} \\ \frac{\partial \phi}{\partial y}\end{array}\right\}$

The boundary conditions of the boundary value problem can be defined in different ways as described in the following sections.

\subsubsection{Dirichlet-Type Boundary Definition on Steel Surface}

In this definition, boundary conditions along the anodic and cathodic surfaces are specified as Dirichlet condition, as shown in Fig. 3.3, and represented as:

$\phi=\phi_{a}$ on $\Gamma_{a}$

$\phi=\phi_{c}$ on $\Gamma_{c}$

$\frac{\partial \phi}{\partial n}=0$ on $\Gamma_{0}$

where $\Gamma_{a}, \Gamma_{c}$ and $\Gamma_{o}$ are the anodic, cathodic and insulated boundaries of the domain

$\Omega$ such that:

$\partial \Omega=\Gamma_{a}+\Gamma_{c}+\Gamma_{o}$

where $\partial \Omega$ is the entire boundary of the boundary value problem. The boundary conditions that are specified in Eqs. 3.25 and 3.26 along the anodic and cathodic surfaces, respectively, are nonlinear. Polarized potential at the anodes, $\phi_{a}$, is given by Eq. 3.19, in which $\phi_{a}$ is a function of the anodic current density, $i_{a}$, which can be calculated by Eq. 3.6. Similarly, polarized potential at the cathodes, $\phi_{c}$, is given by Eq. 3.18, in which $\phi_{c}$ is a function of the cathodic current density, $i_{c}$, which can be calculated by Eq. 3.6. However, Eq. 3.6 requires that the potential distribution within the domain is known, 
hence this requirement results in a non-linear problem.

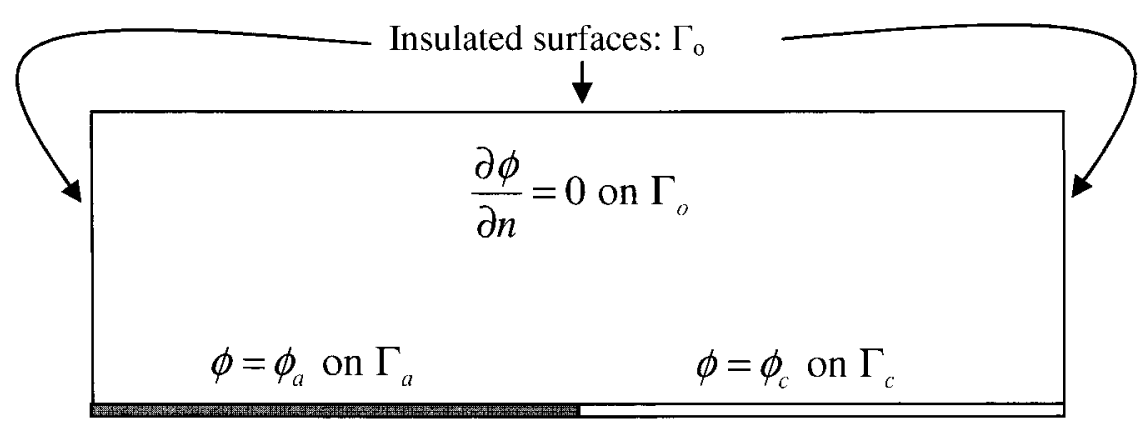

Anode: $\Gamma_{\mathbf{a}}$

Fig. 3.3: Boundary conditions defined as Dirichlet type

\subsubsection{Mixed Dirichlet/Neumann Boundary Definition on Steel} Surface

In the mixed Dirichlet/Neumann boundary definition, the anodic surfaces are specified as Dirichlet condition. As shown in Fig. 3.4, on the cathodic surfaces the flux on the boundary, i.e. Neumann condition, is defined as:

$\phi=\phi_{a}$ on $\Gamma_{a}$

$\frac{\partial \phi}{\partial n}=g_{c}(\phi)$ on $\Gamma_{c}$

$\frac{\partial \phi}{\partial n}=0$ on $\Gamma_{0}$

where $g_{c}(\phi)$ is the cathodic polarization function in Neumann type that establishes the relationship between the polarized potential and the cathodic current density. There are different forms of this relationship proposed in previous studies. Some numerical approximations are also available (Devereux and Yeum 1992]. One of the most common 
models used in the literature is based on Butler-Volmer kinetics (Kranc and Sagues 1992) which can be described as:

$g_{c}(\phi)=i_{L} /\left\{\left(i_{L} / i_{o c}\right) \exp \left[\left(2.303 / \beta_{c}\right)\left(\phi-\phi_{o_{2}}\right)\right]+1\right\}$

where $\phi_{o_{2}}$ is the standard half cell potentials of oxygen. Note that this equation can be obtained from Eq. 3.18 by assuming that:

$\frac{2.303 R T}{z F} \approx \frac{2.303 R T}{\left(1-\alpha_{c}\right) z F}$

As it can be observed from Eqs. 3.29 and 3.30, the boundary conditions that are specified along the anodic and cathodic surfaces are nonlinear. The anodic boundary condition in Eq. 3.29, $\phi_{a}$, is given by Eq. 3.19., in which $\phi_{a}$ is a function of the anodic current density, $i_{a}$, which can be calculated by Eq. 3.6. However, Eq. 3.6 requires that the potential distribution within the domain is known, hence this requirement results in a non-linear problem. Similarly, along the cathodic surfaces the boundary condition is defined by Eq. 3.32, and this relationship requires that the cathodic current densities are known. However, the cathodic current density is a function of the potential distribution within the domain through Eq. 3.6; therefore the cathodic boundary condition also creates nonlinearity. 


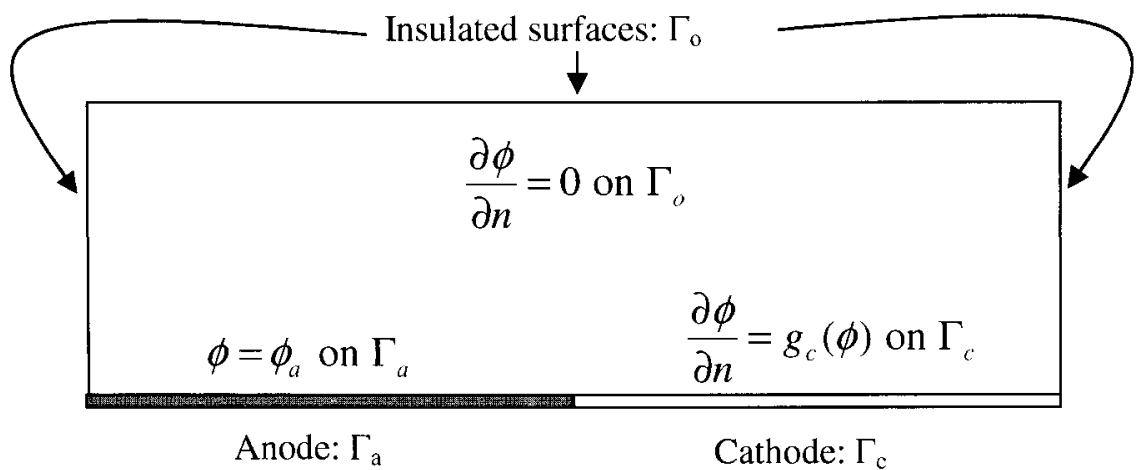

Fig. 3.4: Boundary conditions defined as mixed Dirichlet/Neumann type

It should be noted that the same approach can also be used to model the cathodic sites with Dirichlet condition, and anodic sites with the Neumann condition. The details of this approach will not be provided here.

\subsubsection{Pure Neumann boundary condition}

In the pure Neumann boundary definition, anodic and cathodic surfaces are specified as Neumann condition represented as:

$$
\begin{aligned}
& \frac{\partial \phi}{\partial n}=g_{a}(\phi) \text { on } \Gamma_{a} \\
& \frac{\partial \phi}{\partial n}=g_{c}(\phi) \text { on } \Gamma_{c} \\
& \frac{\partial \phi}{\partial n}=0 \text { on } \Gamma_{o}
\end{aligned}
$$

where $g_{a}(\phi)$ is the anodic polarization function in Neumann type that establishes the relationship between the polarized potential and the anodic current density. It should be noted that this method does not have a unique solution since the integration of the boundary conditions result in infinite integrals such as: 
$\phi=\int g_{x}(\phi)+$ cons tan $t$

Therefore pure Neumann definition along the boundaries is not suitable for the corrosion modelling in concrete. 


\section{Chapter 4}

\section{Finite Element Formulation}

\subsection{General}

To determine the rate of steel corrosion in concrete, the potential distribution in the domain of a corroding system needs to be determined by Laplace's equation as per Eq 3.22, and by the boundary conditions described in Chapter 3. Figure 4.1 illustrates the boundary value problem of a typical corroding system. This chapter explains the details of the finite element implementation to solve this boundary value problem. The formulation presented here is carried out using the Galerkin's weighted residual method (Segerlind 1984).

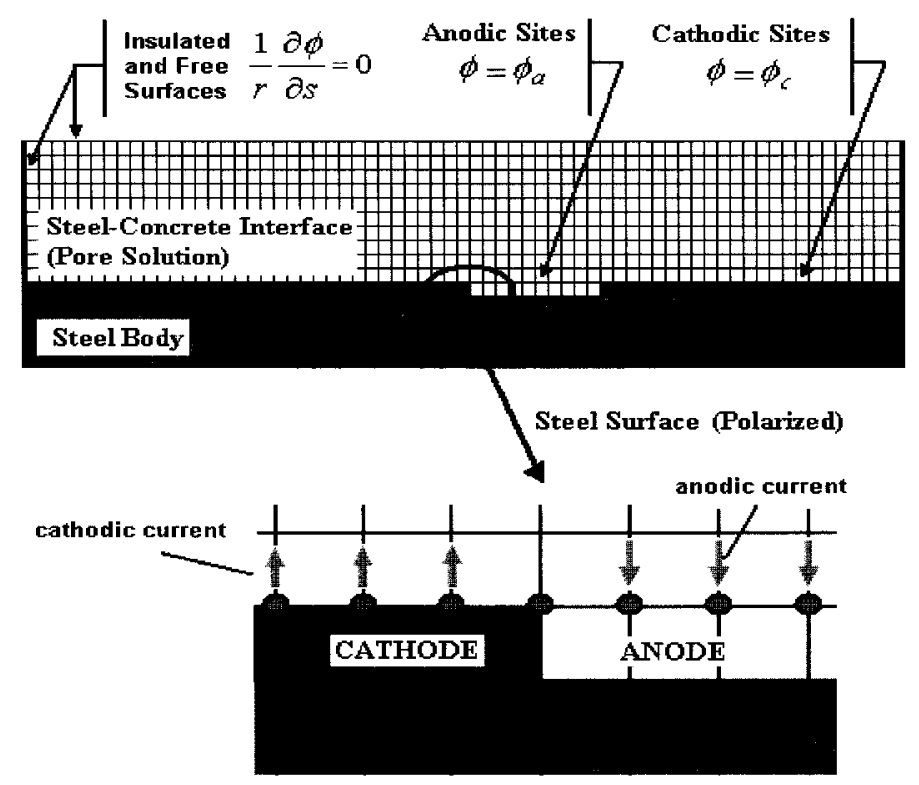

Fig. 4.1: Boundary value problem of a typical corroding system (Source: Isgor 2006) 


\subsection{Finite element formulation}

Based on the Galerkin's method, the vector of residuals, $\{R\}$, of Laplace's equation can be written in integral form as a functional:

$\{R\}=-\int_{\Omega} W(x, y)\left(\frac{\partial^{2} \phi}{\partial x^{2}}+\frac{\partial^{2} \phi}{\partial y^{2}}\right) d \Omega$

where $W(x, y)$ is a weighting function. After discretization of the domain, $\Omega$, the weighted residual integral for each element, $\left\{R^{(e)}\right\}$ represents element (e) matrix of residuals, can be expressed as:

$$
\left\{R^{(e)}\right\}=-\int_{A}[W]^{T}\left(\frac{\partial^{2} \phi}{\partial x^{2}}+\frac{\partial^{2} \phi}{\partial y^{2}}\right) d A
$$

where $A$ is the area representing the domain in two-dimensional coordinate system, and $[W]$ is the weighting functions of individual elements, the superscripts " $T$ " and " $e$ " designate the transpose of the matrix and the variable is defined for an element, respectively. In Galarkin formulation, the weighting functions are selected from the shape functions of the elements; hence Eq. 4.2 can be rewritten as:

$$
\left\{R^{(e)}\right\}=-\int_{A}[N]^{T} \cdot\left(\frac{\partial^{2} \phi}{\partial x^{2}}+\frac{\partial^{2} \phi}{\partial y^{2}}\right) d A
$$

where $[N]$ is the row vector containing the element shape (interpolation) functions. Since $\phi$ may not have continuous derivatives between the elements (i.e. second derivatives may not be defined), the second derivative needs to be replaced by the first derivative terms by applying the product rule for differentiation, Then Eq. 4.3 can be divided into two equations Eq. 4.4 and 4.5: 


$$
\begin{aligned}
& -\int_{A}[N]^{T} \cdot \frac{\partial^{2} \phi}{\partial x^{2}} d A=-\int_{A} \frac{\partial}{\partial x}\left([N]^{T} \cdot \frac{d \phi}{d x}\right) d A+\int_{A} \frac{\partial[N]^{T}}{\partial x} \cdot \frac{\partial \phi}{\partial x} d A \\
& -\int_{A}[N]^{T} \cdot \frac{\partial^{2} \phi}{\partial y^{2}} d A=-\int_{A} \frac{\partial}{\partial y}\left([N]^{T} \cdot \frac{d \phi}{d y}\right) d A+\int_{A} \frac{\partial[N]^{T}}{\partial y} \cdot \frac{\partial \phi}{\partial y} d A
\end{aligned}
$$

Based on Green's Theorem, Eqs. 4.4 and 4.5 can be rewritten as:

$$
\begin{aligned}
& -\int_{A}[N]^{T} \cdot \frac{\partial^{2} \phi}{\partial x^{2}} d A=-\int_{\Gamma}[N]^{T} \cdot \frac{d \phi}{d x} \cdot \cos \theta d \Gamma+\int_{A} \frac{\partial[N]^{T}}{\partial x} \cdot \frac{\partial \phi}{\partial x} d A \\
& -\int_{A}[N]^{T} \cdot \frac{\partial^{2} \phi}{\partial y^{2}} d A=-\int_{\Gamma}[N]^{T} \cdot \frac{d \phi}{d y} \cdot \sin \theta d \Gamma+\int_{A} \frac{\partial[N]^{T}}{\partial y} \cdot \frac{\partial \phi}{\partial y} d A
\end{aligned}
$$

where $\theta$ is the angle between the outward normal of the surface and the x-direction, and $\Gamma$ is the element boundary as shown in Fig. 4.2.

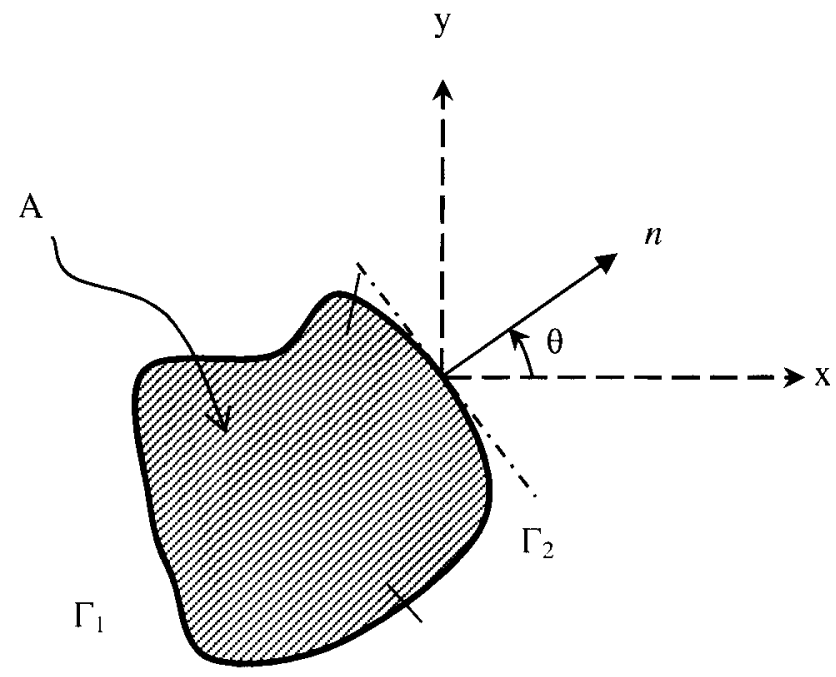

Fig. 4.2: Boundary conditions on a surface element

Substituting Eqs. 4.6 and 4.7 into Eq. 4.3 yields: 


$$
\left\{R^{(e)}\right\}=-\int_{\Gamma}[N]^{T}\left(\frac{\partial \phi}{\partial x} \cdot \cos \theta+\frac{\partial \phi}{\partial y} \sin \theta\right) \cdot d \Gamma+\int_{A}\left(\frac{\partial[N]^{T}}{\partial x} \cdot \frac{\partial \phi}{\partial x}+\frac{\partial[N]^{T}}{\partial y} \cdot \frac{\partial \phi}{\partial y}\right) d A
$$

To solve Eq 4.8, the field variable $\phi$ within an element can be expressed in terms of element nodal values by:

$$
\phi(x, y)=[N] \cdot\left\{\phi^{(e)}\right\}
$$

where $\left\{\phi^{(e)}\right\}$ is the nodal vector for the field variable for a given element. Substituting Eq. 4.9 into Eq. 4.8 yields:

$$
\left\{R^{(e)}\right\}=-\int[N]^{T}\left(\frac{\partial \phi}{\partial x} \cos \theta+\frac{\partial \phi}{\partial x} \sin \theta\right) d \Gamma+\left\{\int\left(\frac{\partial[N]^{T}}{\partial x} \frac{\partial[N]}{\partial x}+\frac{\partial[N]^{T}}{\partial y} \frac{\partial[N]}{\partial y}\right) d A\right\}\left\{\phi^{(e)}\right\}
$$

For the exact solution of Laplace's equation, residuals must be equal to zero; therefore:

$$
-\int_{H}[N]^{T}\left(\frac{\partial \phi}{\partial x} \cos \theta+\frac{\partial \phi}{\partial x} \sin \theta\right) d \Gamma+\left\{\int_{A}\left(\frac{\partial[N]^{T}}{\partial x} \cdot \frac{\partial[N]}{\partial x}+\frac{\partial[N]^{T}}{\partial y} \cdot \frac{\partial[N]}{\partial y}\right) d A\right\}\left\{\phi^{(e)}\right\}=0
$$

Equation 4.11 can also be written in matrix form as:

$$
\left\{I^{(e)}\right\}+\left[K^{(e)}\right]\left\{\Phi^{(e)}\right\}=0
$$

where $\left\{I^{(e)}\right\}$ is the interelement vector:

$$
\left\{I^{(e)}\right\}=-\int_{A}[N]^{T}\left(\frac{\partial \phi}{\partial x} \cos \theta+\frac{\partial \phi}{\partial y} \sin \theta\right) d \Gamma
$$

Since the thesis only deals with Dirichlet type boundary condition this derivative boundary condition (Neumann type boundary condition) is set to zero. $\left[K^{(e)}\right]$ is the element stiffness matrix: 


$$
\left[K^{(e)}\right]=\int_{A}\left(\frac{\partial[N]^{T}}{\partial x} \frac{\partial[N]}{\partial x}+\frac{\partial[N]^{T}}{\partial y} \frac{\partial[N]}{\partial y}\right) d A
$$

Eq. 4.14 can be expressed more compactly by defining:

$$
[B]=\left[\begin{array}{c}
\frac{\partial[N]}{\partial x} \\
\frac{\partial[N]}{\partial y}
\end{array}\right]
$$

Thus the stiffness matrix in Eq. 4.14 can be written as:

$$
\left[K^{(e)}\right]=\int_{A}[B]^{T}[B] d A
$$

In the following, the element stiffness matrix for a rectangular element $(2 b \times 2 a)$ is determined. The element and local coordinates $(s, t)$ are shown in Fig. 4.3, in which $\phi_{i}, \phi_{j}, \phi_{k}$, and $\phi_{m}$ represent the nodal values of the field variable.

Assuming linear interpolation, shape functions, $[N]$, are defined in s-t local coordinate system as (Segerlind 1984):

$$
\begin{aligned}
& N_{i}=\left(1-\frac{s}{2 b}\right)\left(1-\frac{s}{2 a}\right) \\
& N_{j}=\frac{s}{2 b}\left(1-\frac{t}{2 a}\right) \\
& N_{k}=\frac{s t}{4 a b} \\
& N_{m}=\frac{t}{2 a}\left(1-\frac{s}{2 b}\right)
\end{aligned}
$$




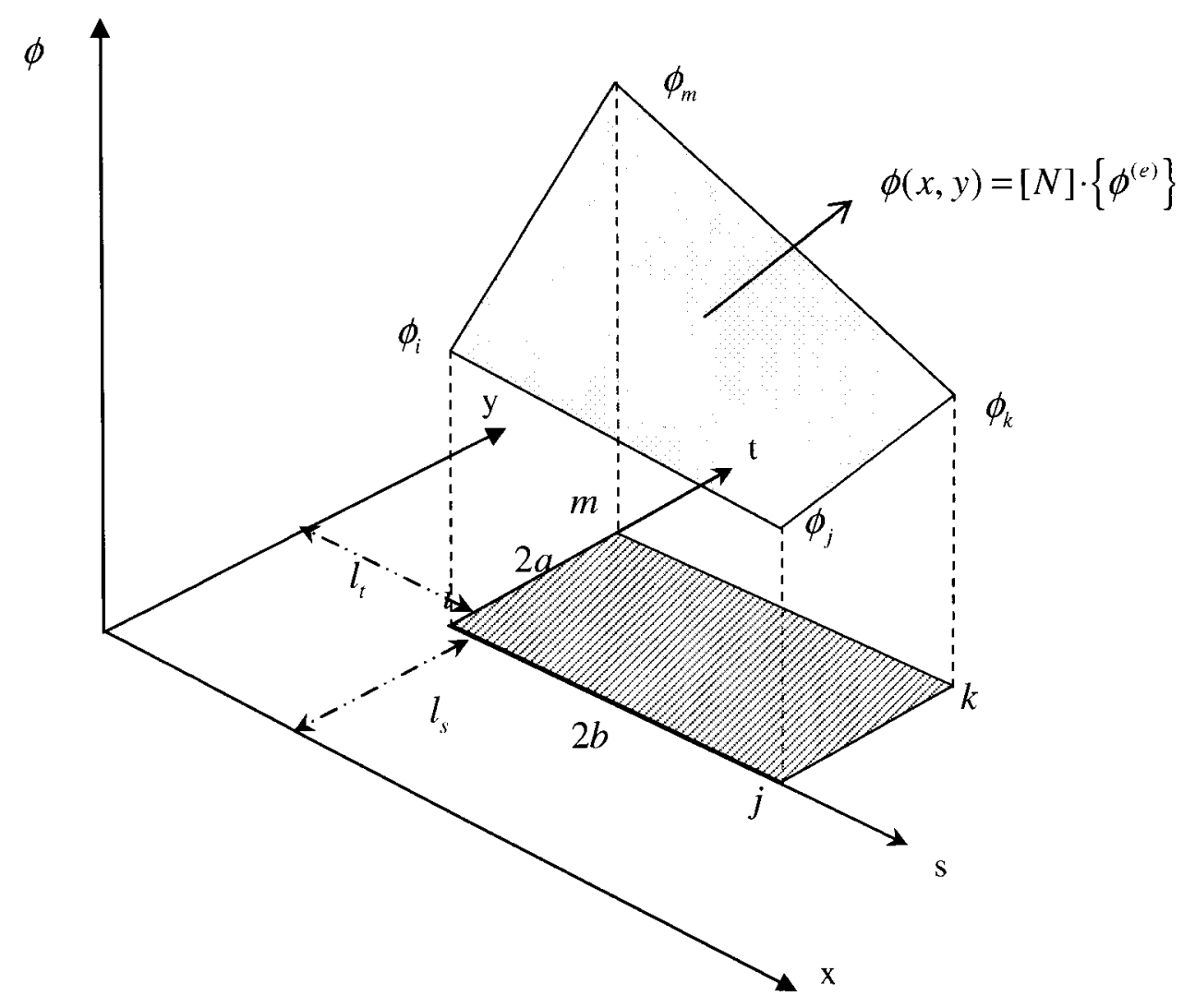

Fig. 4.3: Four-noded rectangular element

The shape functions can also be represented in the Cartesian coordinate system after the following transformation:

$$
\begin{aligned}
& x(s, t)=s+l_{s} \\
& y(s, t)=t+l_{t}
\end{aligned}
$$

where $l_{s}$ and $l_{t}$ are the distances of the origin of the element from $s$ and $t$ axes, respectively.

Since the Cartesian coordinate system is parallel to local coordinate system, and the unit lengths in both coordinate systems are the same (i.e. Jacobian =1) (Segerlind 1984), any 
integral represented in local coordinate system can be written in the Cartesian coordinate system as:

$$
\int_{A} f(x, y) d x \cdot d y=\int_{A} g(s, t) d s \cdot d t
$$

where $f(\mathrm{x}, \mathrm{y})$ and $g(\mathrm{~s}, \mathrm{t})$ represent the same $\mathrm{x}-\mathrm{y}$ and s-t coordinate systems, respectively.

The derivatives of the shape functions in the $x-y$ coordinate system can be obtained by the chain rule:

$$
\begin{gathered}
\frac{\partial N_{\text {node }}}{\partial x}=\frac{\partial N_{\text {node }}}{\partial s} \frac{\partial s}{\partial x}=\frac{\partial N_{\text {node }}}{\partial s} \frac{\partial\left(x-l_{S}\right)}{\partial x}=\frac{\partial N_{\text {node }}}{\partial s} \\
\frac{\partial N_{\text {node }}}{\partial y}=\frac{\partial N_{\text {node }}}{\partial t} \frac{\partial t}{\partial y}=\frac{\partial N_{\text {node }}}{\partial t} \frac{\partial\left(y-l_{t}\right)}{\partial y}=\frac{\partial N_{\text {node }}}{\partial t}
\end{gathered}
$$

where $N_{\text {node }}$ is the value of a shape function at a given node. The derivative matrix, $[B]$, can be rewritten by substituting Eqs. 4.24 and 4.25 into Eq. 4.15 :

$$
[B]=\left[\begin{array}{cccc}
\frac{\partial N_{i}}{\partial s} & \frac{\partial N_{j}}{\partial s} & \frac{\partial N_{k}}{\partial s} & \frac{\partial N_{m}}{\partial s} \\
\frac{\partial N_{i}}{\partial t} & \frac{\partial N_{j}}{\partial t} & \frac{\partial N_{k}}{\partial t} & \frac{\partial N_{m}}{\partial t}
\end{array}\right]
$$

Substituting Eqs. 4.17 to 4.20 into Eqs. 4.24 and 4.25 , the derivative matrix can be expressed as:

$$
[B]=\frac{1}{4 a b}\left[\begin{array}{cccc}
-(2 a-t) & 2 a-t & t & -t \\
-(2 b-s) & -s & s & 2 b-s
\end{array}\right]
$$

If Eq. 4.27 is substituted in Eq. 4.22, the stiffness matrix can be rewritten as: 


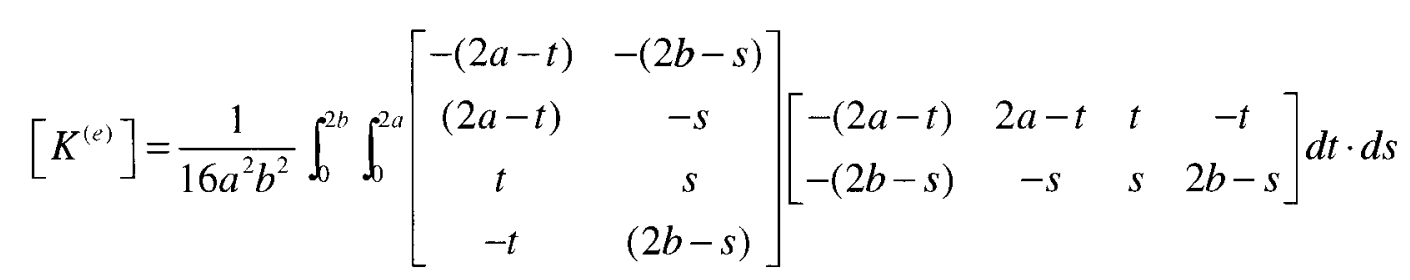

After integrating and rearranging:

$$
\left[K^{(e)}\right]=\frac{a}{6 b}\left[\begin{array}{cccc}
2 & -2 & -1 & 1 \\
-2 & 2 & 1 & -1 \\
-1 & 1 & 2 & -2 \\
1 & -1 & -2 & 2
\end{array}\right]+\frac{b}{6 a}\left[\begin{array}{cccc}
2 & 1 & -1 & -2 \\
1 & 2 & -2 & -1 \\
-1 & -2 & 2 & 1 \\
-2 & -1 & 1 & 2
\end{array}\right]
$$

The derivatives of the field variable that are used in the calculation of the flux terms can be determined using:

$$
\frac{\partial \phi^{(e)}}{\partial n}=\left\{\begin{array}{l}
\frac{\partial \phi}{\partial x} \\
\frac{\partial \phi}{\partial y}
\end{array}\right\}=\left[\begin{array}{l}
\frac{\partial\left([N]\left\{\phi^{(e)}\right\}\right)}{\partial x} \\
\frac{\partial\left([N]\left\{\phi^{(e)}\right\}\right)}{\partial y}
\end{array}\right]=[B]\left\{\phi^{(e)}\right\}
$$

Following Eq. 4.30, at the centre of the element where $t=a$ and $s=b$

$$
\frac{\partial \phi^{(e)}}{\partial n}=\frac{1}{4 a b}\left[\begin{array}{cccc}
-(2 a-t) & 2 a-t & t & -t \\
-(2 b-s) & -s & s & 2 b-s
\end{array}\right]\left\{\begin{array}{c}
\phi_{i} \\
\phi_{j} \\
\phi_{k} \\
\phi_{m}
\end{array}\right\}=\left[\begin{array}{cc}
\frac{\phi_{j}+\phi_{k}}{4 b}-\frac{\phi_{i}+\phi_{m}}{4 b} \\
\frac{\phi_{k}+\phi_{m}}{4 a}-\frac{\phi_{i}+\phi_{j}}{4 a}
\end{array}\right]
$$

Substituting Eq. 4.31 into Eq. 3.23, the current density of an element, $i^{(e)}$, can be expressed by:

$$
i^{(e)}=-\frac{1}{r}\left[\begin{array}{cc}
\frac{\phi_{j}+\phi_{k}}{4 b}-\frac{\phi_{i}+\phi_{m}}{4 b} \\
\frac{\phi_{k}+\phi_{m}}{4 a}-\frac{\phi_{i}+\phi_{j}}{4 a}
\end{array}\right]
$$

The nodal values of the current density can be calculated by averaging the current 
densities of adjacent elements:

$i_{\text {node }}=\frac{i^{(e)}+i^{(e-1)}}{2}$

After assembling the element matrix to a global matrix, Eq. 4.12 becomes

$[K]\{\Phi\}=0$

where $[K]$ is the global stiffness matrix and $\{\Phi\}$ is the global variable matrix of variables. Since the global variable matrix of variables consist of known values of $\phi_{b}$ on the boundary, $\{\Phi\}$ can be represented by:

$$
\{\Phi\}=\left\{\begin{array}{c}
\phi_{b} \\
\Phi^{(e)}
\end{array}\right\}
$$

where $\phi_{b}$ is the Dirichlet-type nodal boundary values. If Eq. 4.35 is substituted in Eq. 4.34:

$$
[K]\left\{\begin{array}{c}
\phi_{b} \\
\Phi^{(e)}
\end{array}\right\}=0
$$

Rearranging Eq. 4.36 yields:

$$
\left[k^{(e)}\right]\left\{\Phi^{(e)}\right\}=\left[k_{b}^{(e)}\right]\left\{\phi_{b}\right\}
$$

where $\left[k^{(e)}\right]$ is the element stiffness matrix and $\left[k_{b}^{(e)}\right]$ is the element boundary matrix. The left side of Eq. 4.37 is the force vector that drives the problem, and the non-linearity of the solution is introduced through this term, since $\phi_{b}$ is equal to the anodic polarized potential, $\phi_{a}$, or cathodic polarized potential, $\phi_{c}$, both being functions of the current density as per Eqs. 3.18 and 3.19, respectively. 


\subsection{Non-linear solution algorithms}

\subsubsection{Direction iteration method (Pichard iteration)}

One of the methods that can be used to solve the nonlinear boundary value problem defined in Eq. 4.37, for the calculation of corrosion rates is the direct iteration method (DIM) (Dhatt 1984). The main advantage of the DIM is that it is applicable to all boundary condition definitions described in Chapter 3 . Here, the method will be described for a Dirichlet-type boundary definition since all analyses in this study have been carried out using Dirichlet-type boundary conditions.

With reference to the flowchart in Fig.4.4, DIM consists of constructing a series of solutions $\left\{\phi^{0}\right\},\left\{\phi^{1}\right\},\left\{\phi^{2}\right\}, \cdots,\left\{\phi^{k}\right\}$, where $\left\{\phi^{k}\right\}$ is calculated from the previous value $\left\{\phi^{k-1}\right\}$, where the superscript, $k$, represents the iteration number. The solution starts with the assumption of values for all potentials on the steel surface, $\left\{\phi^{0}\right\}$. These initial potentials can be calculated by substituting small values of current densities, $i_{a}$ and $i_{c}$ as given by Eqs. 3.18 and 3.19, respectively. The solution of Eq. 3.22 with imposed values of the initial potentials at the cathodic and anodic surfaces of steel rebar yields a potential distribution within the domain. The potential gradients at the steel concrete interface that are obtained form this distribution are used to calculate new current densities by using Eq.3.23, and then the calculated current densities, $i_{a}$ and $i_{c}$, are respectively substituted in Eqs. 3.18 and 3.19 to update the new potentials on the steel surface. With these new boundary conditions, Eq. 3.23 can be solved again to obtain a new set of potentials in the 
domain. At the end of each solution, the calculated nodal values of the potential are compared with the corresponding values in the previous iteration to check for convergence. Iterations are terminated when a user-defined convergence norm is satisfied. Figures 4.5 and 4.6 show the DIM convergence process based on anode and cathode polarization, respectively.

The basic working principle of the DIM is illustrated in Fig. 4.5. It should be noted that Fig.4.5 shows an ideal case where the solution converges to the expected result with successive iterations. Even though the DIM is easy to implement, it has significant limitations for complex problems such as reinforcement corrosion in concrete structures; it generally fails to provide converged and accurate results. A typical example of divergence in DIM is illustrated in Fig. 4.7 through steps 0-5. More numerical simulations by DIM are provided in Appendix 2. 


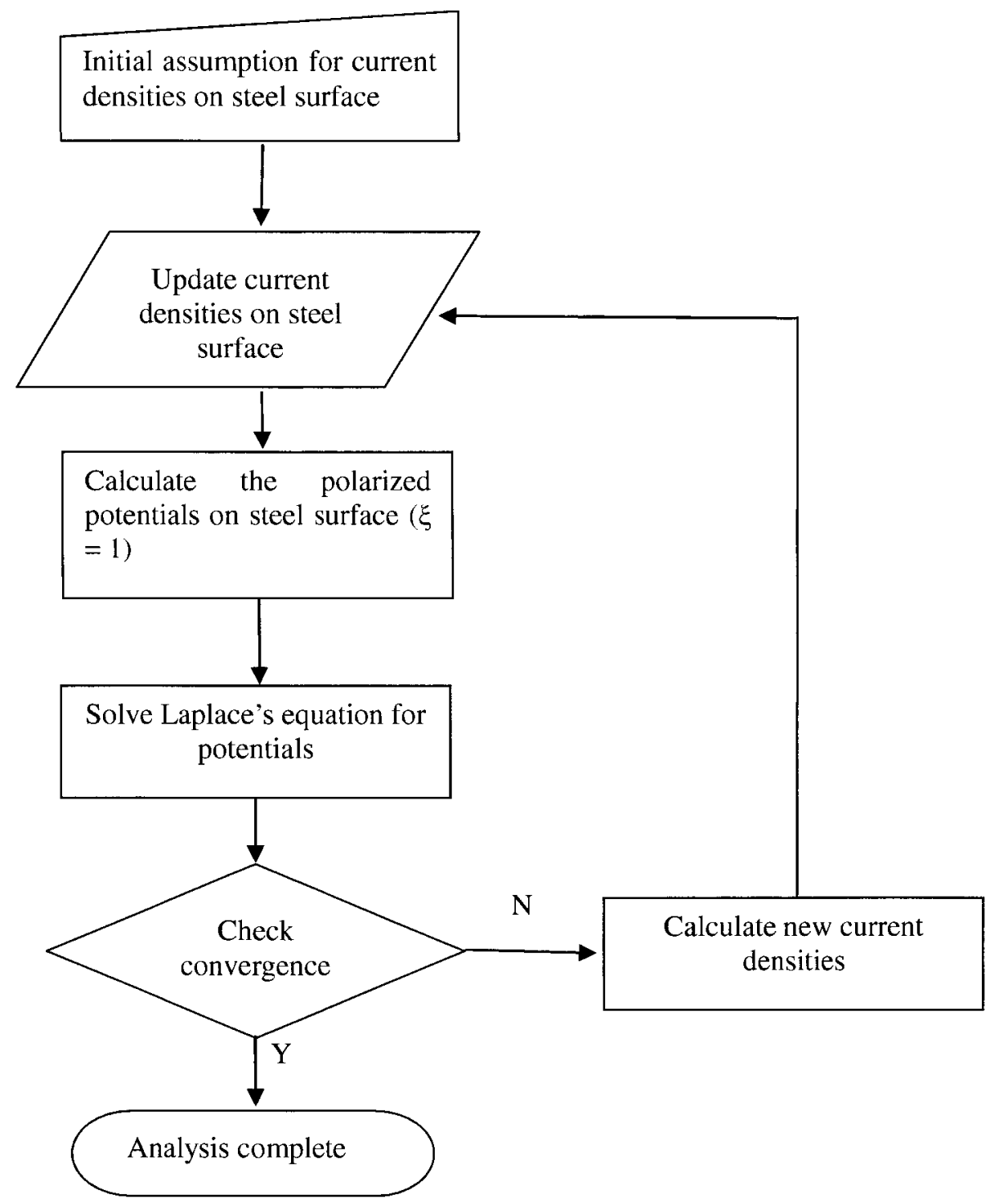

Fig. 4.4: Flowchart of the DIM 


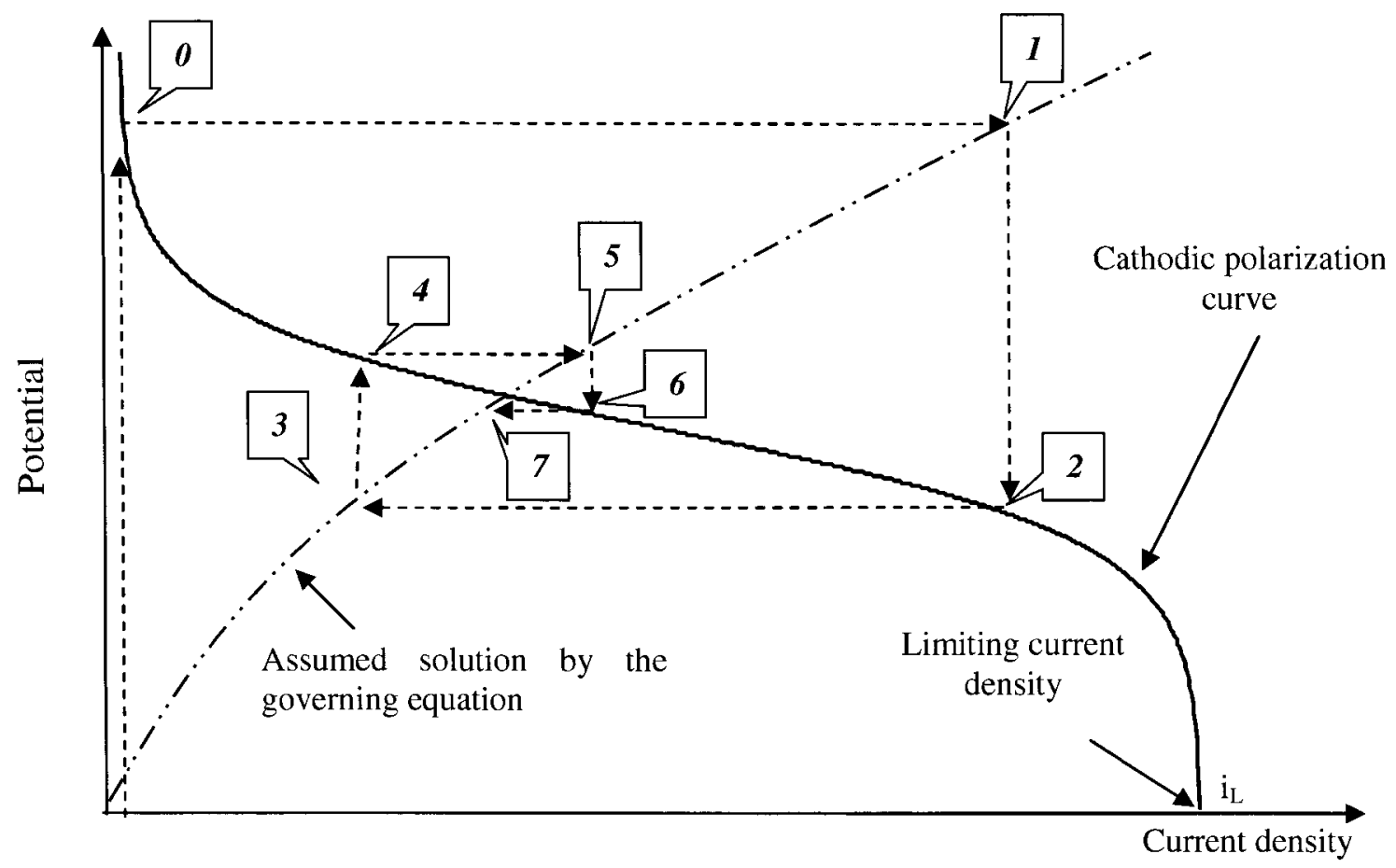

Fig. 4.5: Converged solution using the DIM at a cathodic node

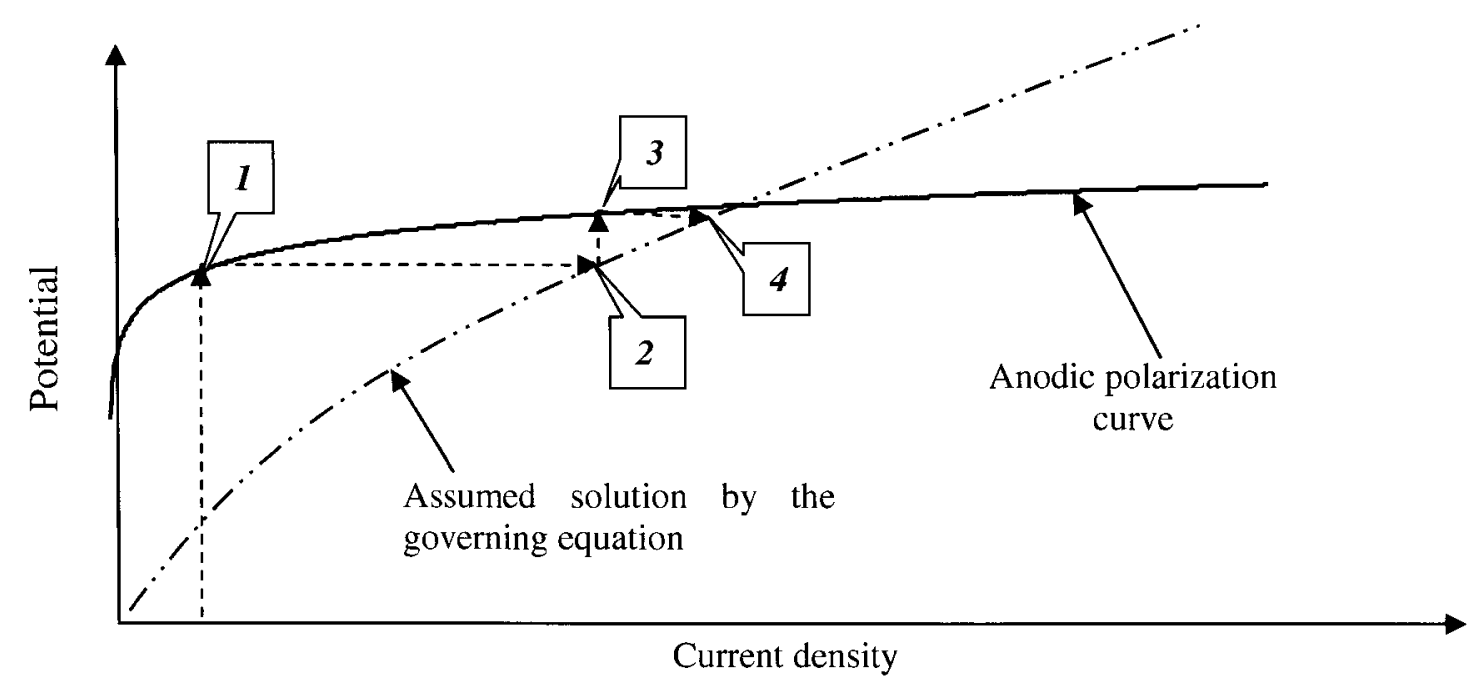

Fig. 4.6: Converged solution using the DIM at an anodic node 


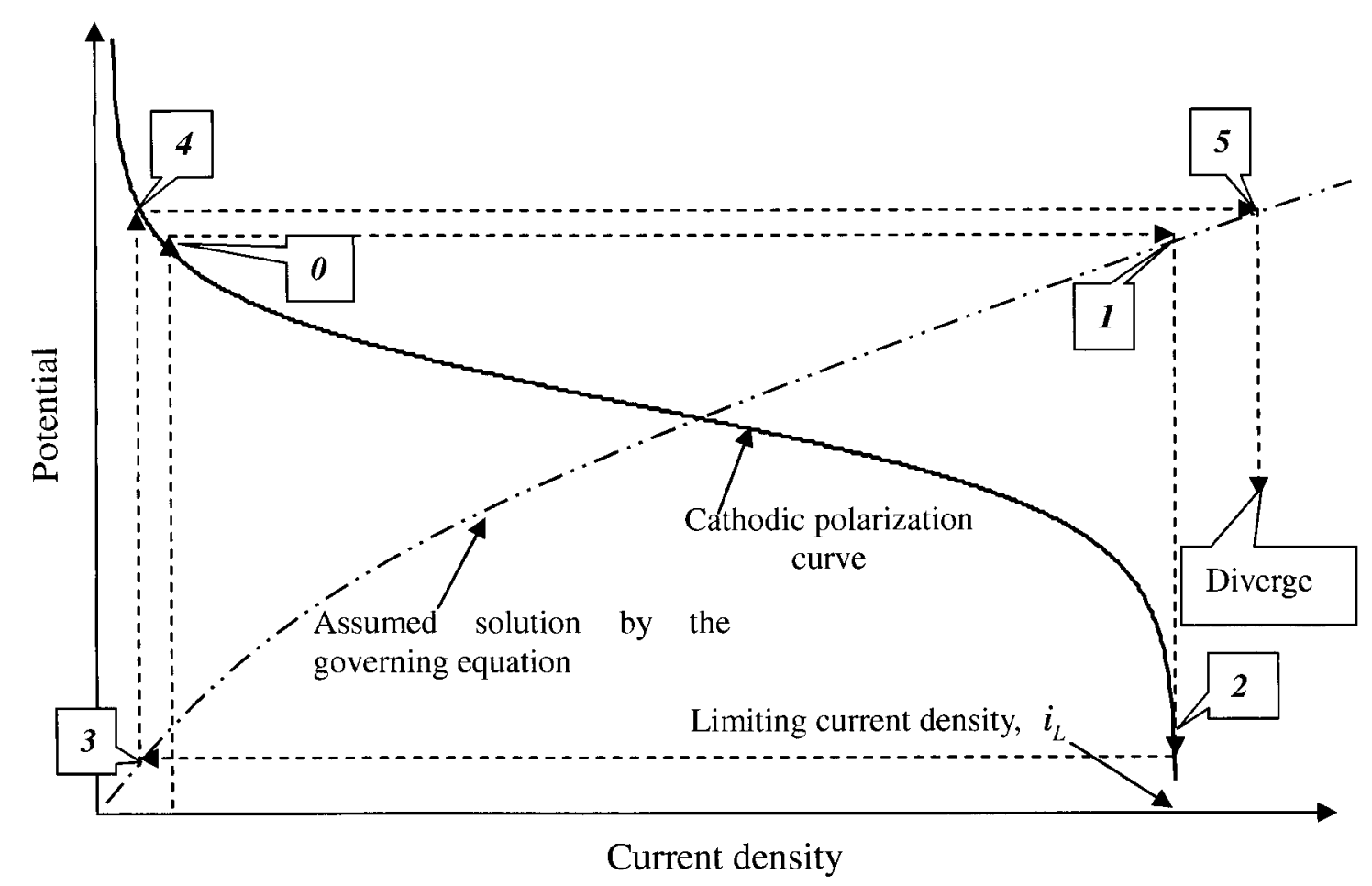

Fig. 4.7: Diverged solution by DIM at a cathodic node

Another limitation of the DIM is the fact that when the limiting current density is very small, (e.g. $i_{L}<10^{-8} \mathrm{~A} / \mathrm{mm}^{2}$ ), it can violate the limitation imposed in the polarization of the cathodic reaction. Starting from the first iteration, the current density calculated from the solution of Laplace's equation can be larger than the limiting current density. When this happens, the solution will not converge, as illustrated in Fig. 4.7. This problem is more significant when the oxygen supply around the reinforcement is limited, leading to smaller values of limiting current density as per Eq. 3.15 


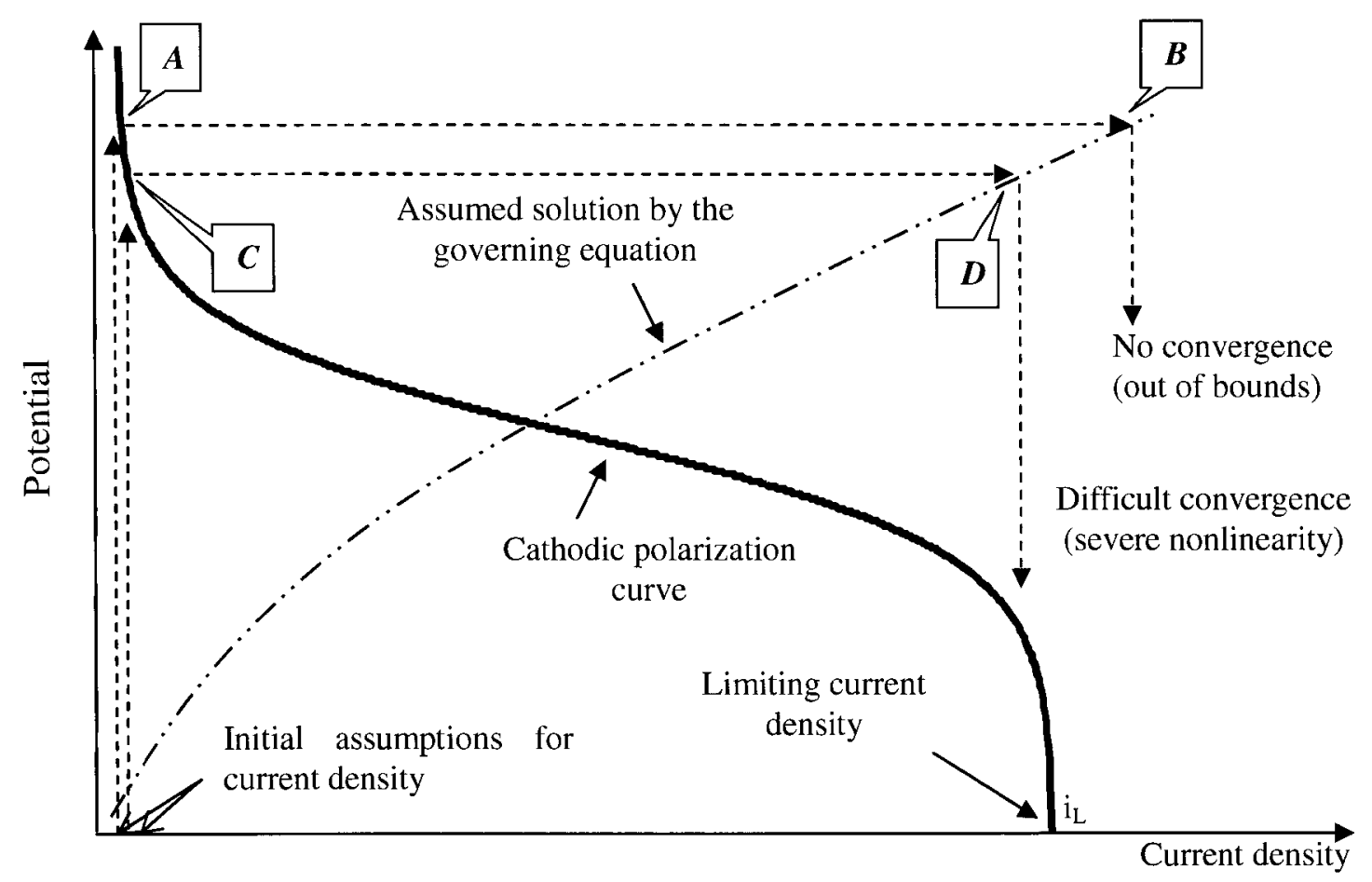

Fig. 4.8: The effect of limiting current density on the convergence at a cathodic node

\subsubsection{Modified direction iteration method (MDIM)}

In order to solve the problems associated with the limitations of the DIM, a new method, called the modified direct iteration method (MDIM), is developed in the current study. As shown in Fig. 4.9, the MDIM uses a similar iterative approach as the DIM, albeit with an under-relaxation factor, $\xi$, and a limiting current density control factor, $\psi$. In the MDIM, the boundary conditions on the steel surface, assuming Dirichlet-type definition, are determined as:

$\phi^{k+1}=(1-\xi) \phi^{k}+\xi f\left(i^{k}\right)$

where $\xi$ is an under-relaxation factor that varies between 0 and 1 . The new boundary condition $\phi^{k+1}$ is calculated as a weighted average of the boundary condition of the previous iteration and the boundary condition provided by the polarization equation, 
$f\left(i^{k}\right)$. The MDIM has an inherent measure of trust that the new iterate is moving towards a converged solution. It should be noted that when $\xi=1$, the MDIM is the same as the DIM. The limiting current density control factor is used when the calculated current density at a given point on the steel surface exceeds the limiting current density as shown in Fig 4.8. The limiting current density correction factor is also a value between 0 and 1 , but its selection depends on the amount of difference between the calculated current density at a given node and the limiting current density.

In Fig. 4.10, the application of the MDIM is demonstrated for two cathodic nodes. Iterations that take place for Node 1 require only the under-relaxation factor, since current densities obtained after each iteration do not exceed the limiting current density, $i_{L}$; hence, an under-relaxation factor is adequate to reach convergence at this node. However, it can be observed from Fig. 4.10 that at Node 2, after the first iteration, the limiting current density, $i_{L}$, is exceeded; therefore a limiting current density correction factor is needed. For Node 2, implementation of both factors is necessary for convergence. 


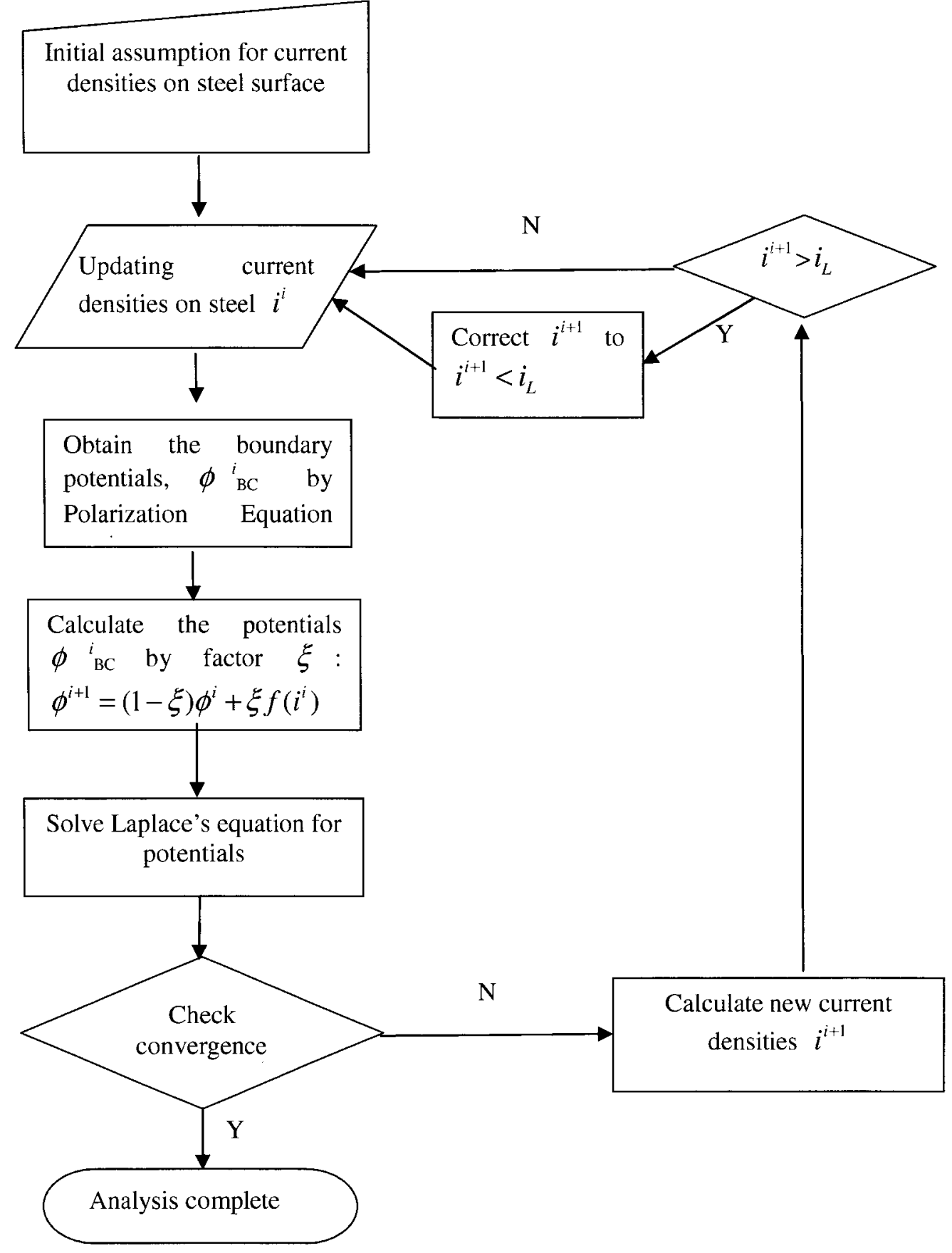

Fig. 4.9: Flowchart of the MDIM 


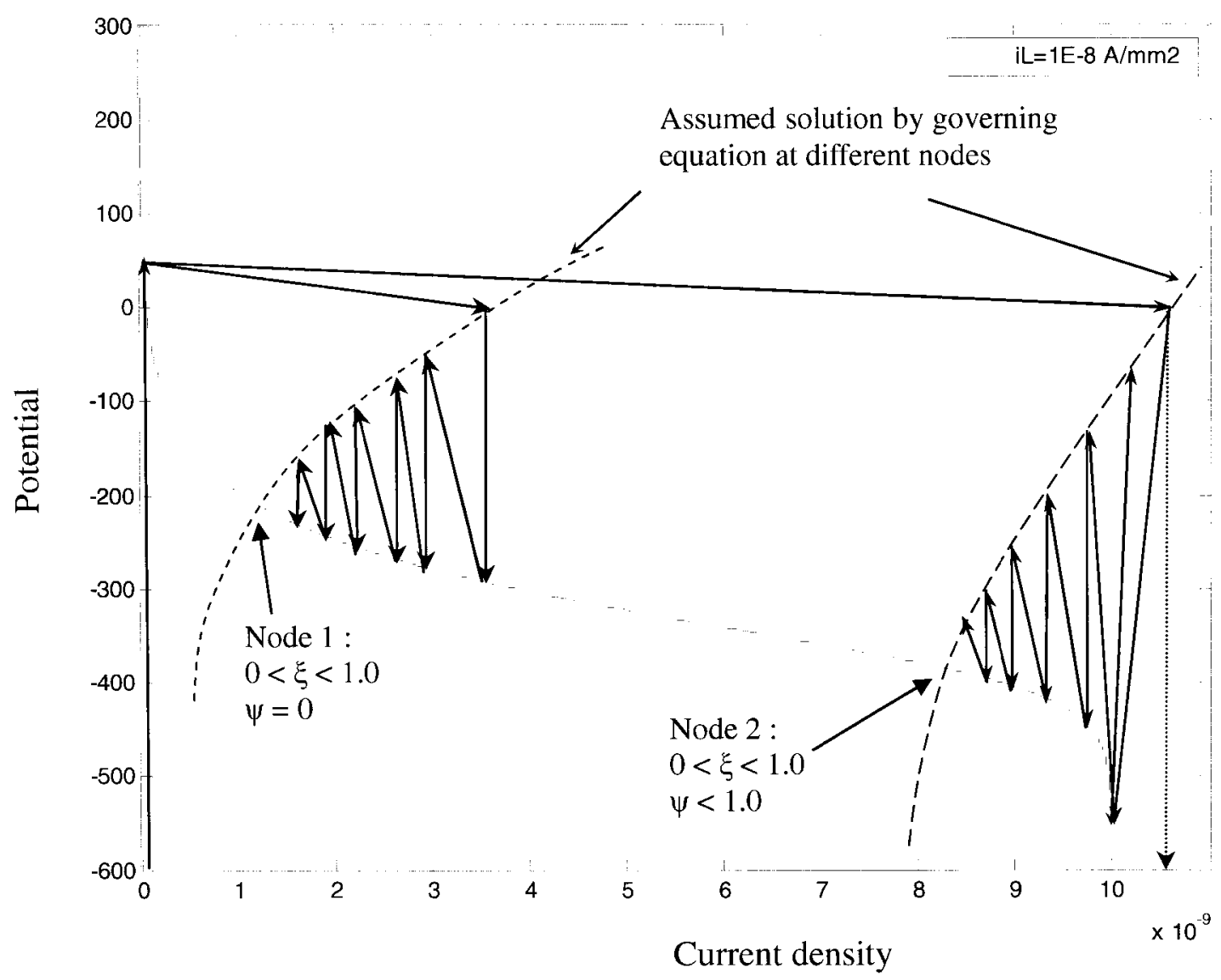

Fig. 4.10: Solution using the MDIM at two different cathodic nodes

\subsection{Convergence criterion}

The convergence of the nonlinear solution algorithms used in this investigation is checked by the "maximum difference" method. At the end of each iteration, the difference between the nodal values of potential in the current and previous iterations is calculated, and the maximum value of these values is determined. This maximum difference is called "maximum error" in this thesis. It should be noted that the term "error" here does not 
imply the difference between the numerical and experimental (or theoretical) results, but only refers to the difference between two successive iterations.

\subsection{Developed computer program}

The analyses presented in this thesis were carried out using a Windows-based computer program developed for this investigation. The program consists of the following components: (1) Graphical user interface (GUI); (2) Engineering module, which contains the numerical algorithms presented in this thesis and communicates with the finite element engine, CONDUR developed by Isgor (2001); (3) Interface module between GUI and Matlab for graphical presentation of results. A flowchart representing the interactions between different modules is presented in Fig. 4.11. Screenshots of the program can be seen in the Appendix of this thesis.

GUI has been developed using Microsoft Visual Basic 2003.NET with object oriented programming techniques. Since most engineering quantities are stored in double precision format, the program runs on $128 \mathrm{MB}$ (or greater) RAM. Although the program requires only $4 \mathrm{MB}$ of hard disk space for installation, due to the large size of temporary and output files, at least $50 \mathrm{MB}$ of free space is recommended. The following basic user operations are presented in Appendix A to introduce the developed program:

- Geometrical data entry (see Fig. A1);

- Environmental parameter entry (see Fig. A2); 
- Corrosion parameter entry (see Fig. A3)

- Boundary condition entry (see Fig. A4-A6);

- Analysis option entry (see Fig. A7);

- Outputting results (see Fig. A8);

- Saving data files (see Fig. A9).

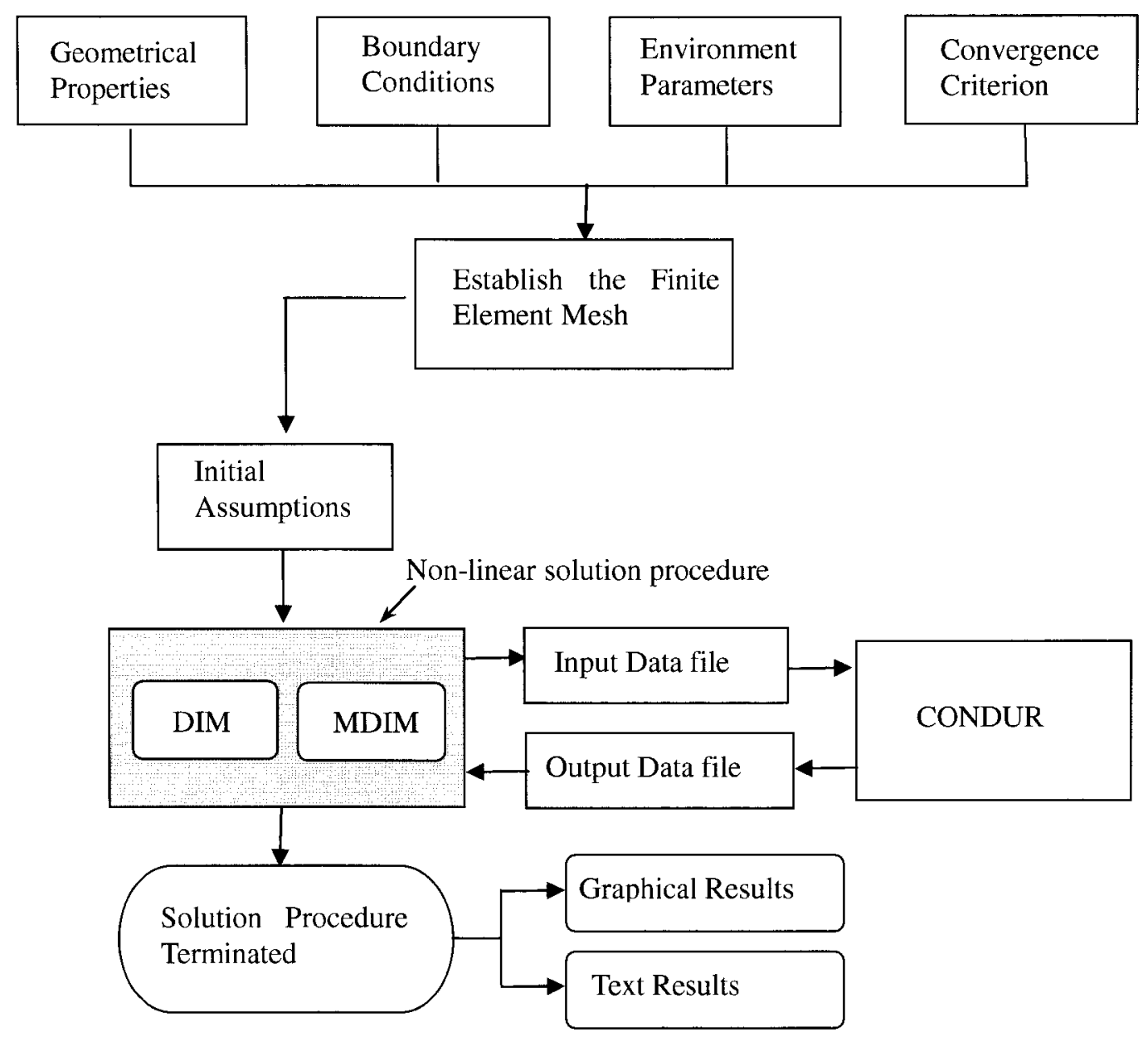

Fig. 4.11: Flowchart of the developed program 


\section{Chapter 5}

\section{Numerical Investigation}

\subsection{General}

In this chapter, the efficiency of the numerical algorithms for the solution of Laplace's equation with nonlinear polarized boundary conditions is investigated in detail. In particular, the convergence of solution is studied under various conditions, which are commonly encountered in the modelling of steel corrosion in concrete.

It is well documented (Isgor and Razaqpur 2006, Ghods et al. 2006, Pour-Ghaz et al. 2006) that steel corrosion in concrete is sensitive to the following properties: (1) Anode-to-cathode ratio (A/C ratio); (2) Concrete (pore solution) resistivity; (3) Amount of oxygen that is present around the steel (i.e. limiting current density). In addition to these, the fineness of finite element mesh, i.e. element size, and the initial conditions are known to be an important parameter that affects the convergence of nonlinear problems. Therefore the effect of these parameters on the efficiency of the algorithms presented in Chapter 4 is presented here.

The investigation starts with the demonstration of the issues associated with the DIM. Later, the solution by the MDIM is presented in a number of stages. Firstly, a detailed 
study is carried out on the selection of the under-relaxation and limiting current density correction factors. Following this, the efficiency of the MDIM is investigated with varying element sizes, $\mathrm{A} / \mathrm{C}$ ratios, and concrete resistivity. When the effect of one parameter is studied, all other parameters are kept constant at base-case conditions. The base case is explained in detail in the following section. The effect of limiting current density is incorporated by analyzing each case at three levels of oxygen availability around the steel: (1) Large oxygen concentration, i.e. limiting current density is greater than $10^{-6} \mathrm{~A} / \mathrm{mm}^{2}$; (2) Average oxygen concentration, i.e. limiting current density is between $10^{-7}-10^{-6} \mathrm{~A} / \mathrm{mm}^{2}$; (3) Low oxygen concentration, i.e. limiting current density is less than $10^{-8} \mathrm{~A} / \mathrm{mm}^{2}$.

\subsection{Test model and the base case data}

The test model used in this numerical investigation consists of a steel rebar embedded in a prismatic concrete member as shown in Fig. 5.1. The middle region of steel is assumed to be anodic (length: 2a), while the rest remains cathodic (length: $2 b$ ). The dimensions of the test model are selected such that the size of the domain does not have any influence on the convergence of the solution. To ensure this, a number of cases with different dimensions have been run, and it was determined that a reinforced concrete member with dimensions $300 \times 600 \mathrm{~mm}$ is large enough to eliminate possible size effect problems and small enough to avoid expensive numerical computations. The arrangement of the anodic and cathodic regions on the steel surface represents typical macro-cell corrosion behaviour on steel surface, which is a very useful case commonly encountered in 
corrosion modelling studies in concrete.

Using symmetry, only the upper-right quarter of the member is modeled throughout this study. To simplify the problem, the concrete is treated as a homogenous medium with uniform resistivity. Therefore the domain of interest is a $100 \times 300 \mathrm{~mm}$ rectangular area with all boundaries, except for the lower edge facing the steel surface, modelled as insulated boundary conditions. On the steel surface, the anode and cathode are adjacent to each other with lengths $a$ and $b$, respectively. Table 5.1 provides the base case input parameters that are used in the analyses carried out in this numerical investigation. It should be noted that some of the parameters in Table 5.1 are varied to study their effect on the numerical solution; these varied values will be provided when the corresponding analysis is presented.

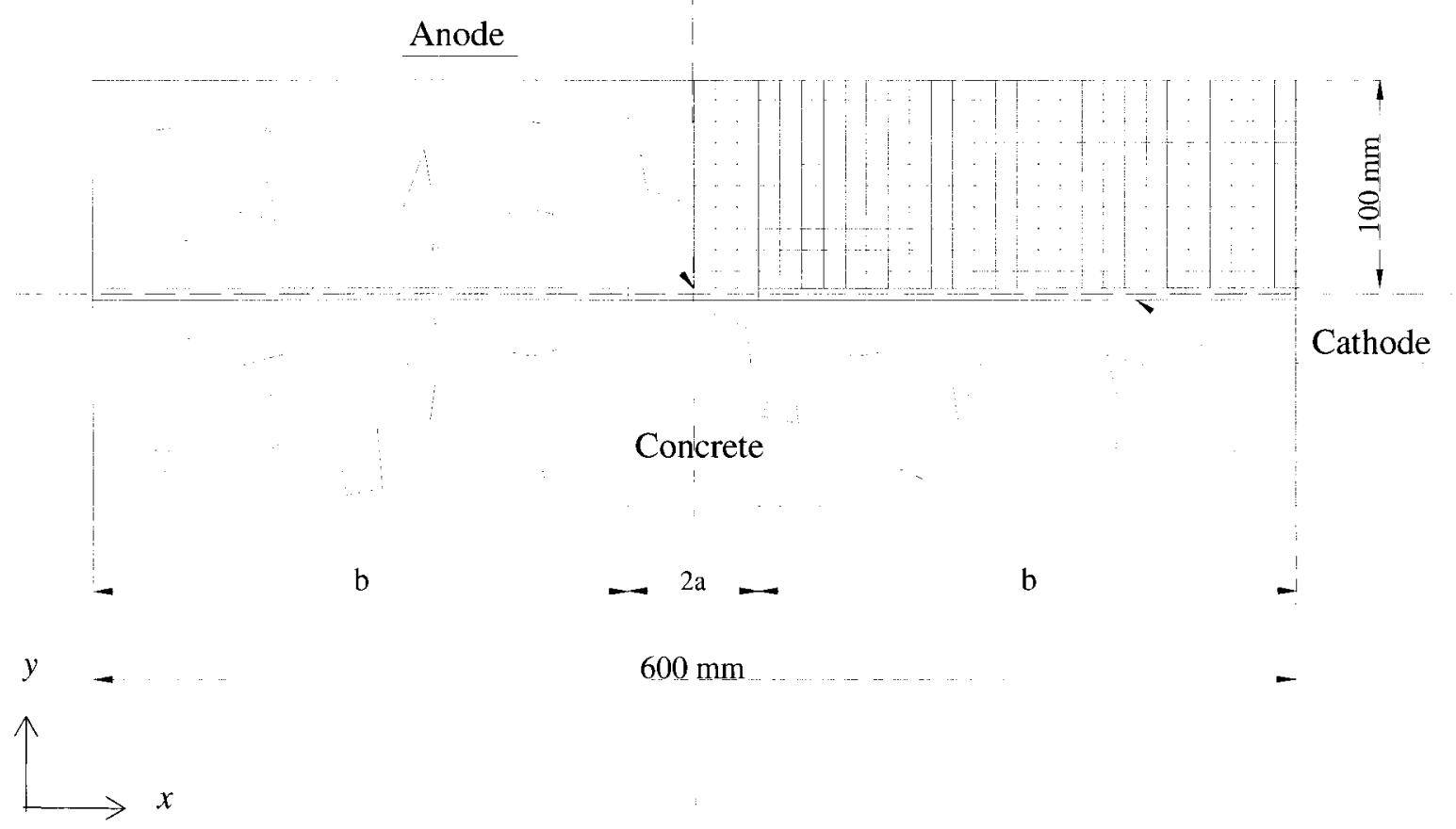

Fig. 5.1: Test model used in the present numerical investigation 
Table 5.1: Input parameters of the base case

\begin{tabular}{|c|c|}
\hline Parameter & Value \\
\hline Length, $a+b(\mathrm{~mm})$ & 300 \\
\hline Height (mm) & 100 \\
\hline Anode length, a (mm) & 30 \\
\hline Element size $(\mathrm{mm})$ & 5 \\
\hline Standard half cell potential of $\mathrm{Fe}\left(\mathrm{mV} \sim \mathrm{SCE}^{*}\right)$ & -780 \\
\hline Tafel slope of the anode, $\beta_{a}(\mathrm{mV})$ & 60 \\
\hline Anodic exchange current density, $i_{o a}\left(\mathrm{~A} / \mathrm{mm}^{2}\right)$ & $187.5 \times 10^{-12}$ \\
\hline Standard half cell potential of oxygen $\left(\mathrm{mV} \sim \mathrm{SCE}^{*}\right)$ & 160 \\
\hline Tafel slope of the cathode, $\beta_{c}(\mathrm{mV})$ & 160 \\
\hline Cathodic exchange current density, $\quad i_{o c}\left(\mathrm{~A} / \mathrm{mm}^{2}\right)$ & $6.25 \times 10^{-12}$ \\
\hline Temperature, $T(\mathrm{~K})$ & 298 \\
\hline Resistivity, $r$ (ohm-mm) & 140,000 \\
\hline Limiting current density, $i_{L}\left(\mathrm{~A} / \mathrm{mm}^{2}\right)$ & $1 \times 10^{-6}$ \\
\hline
\end{tabular}

$\left.{ }^{*}\right)$ The measurement is made with respect to standard calomel electrode (SCE)

\subsection{Solution by direct iteration method (DIM)}

In order to demonstrate the issues associated with the DIM, the base case, whose data is provided in Table 5.1, is run to investigate the effects of the following parameters on the numerical solution: (1) element size, (2) A/C ratio; (3) resistivity of concrete. To eliminate the possible convergence problems that may originate from the limiting current density, it is assumed that adequate amount of oxygen is present around the reinforcement. Therefore the limiting current density used in the analysis is selected as $1 \times 10^{-6} \mathrm{~A} / \mathrm{mm}^{2}$, which is large enough to ensure that the convergence issues are not a result of the limiting current density.

When a convergence norm of $0.1 \mathrm{mV}$ is used to check convergence at every node, it is observed that none of the analysis cases converges. Therefore only the best results, the 
case in which the maximum error is the smallest, are presented here by allowing the analysis run to a maximum of 5000 iterations.

\subsubsection{Element size analysis}

The base case is run with square elements with the following sizes: $2 \mathrm{~mm}, 5 \mathrm{~mm}, 10 \mathrm{~mm}$ and $15 \mathrm{~mm}$. The smallest values of the maximum error after 5000 iterations are found to be $87.1 \mathrm{mV}, 71.7 \mathrm{mV}$ and $58.2 \mathrm{mV}$ for $5-\mathrm{mm}, 10-\mathrm{mm}$ and $15-\mathrm{mm}$ elements, respectively. The case with 2-mm elements provides a maximum error greater than $100 \mathrm{mV}$; therefore its results are not shown in Figs. 5.2 and 5.3, which illustrate the potential distribution and current density on the steel surface for the iterations corresponding to the smallest values of the maximum error. It can be observed from this figure that, besides their large maximum error values (i.e. no convergence), there are significant fluctuations in potential distribution and current density, especially along the cathodic regions of the reinforcement. A sample contour plot in Fig. 5.4 for the 5-mm element shows that the distribution of potentials in the domain is not smooth, which illustrates the reason of the convergence problem. 


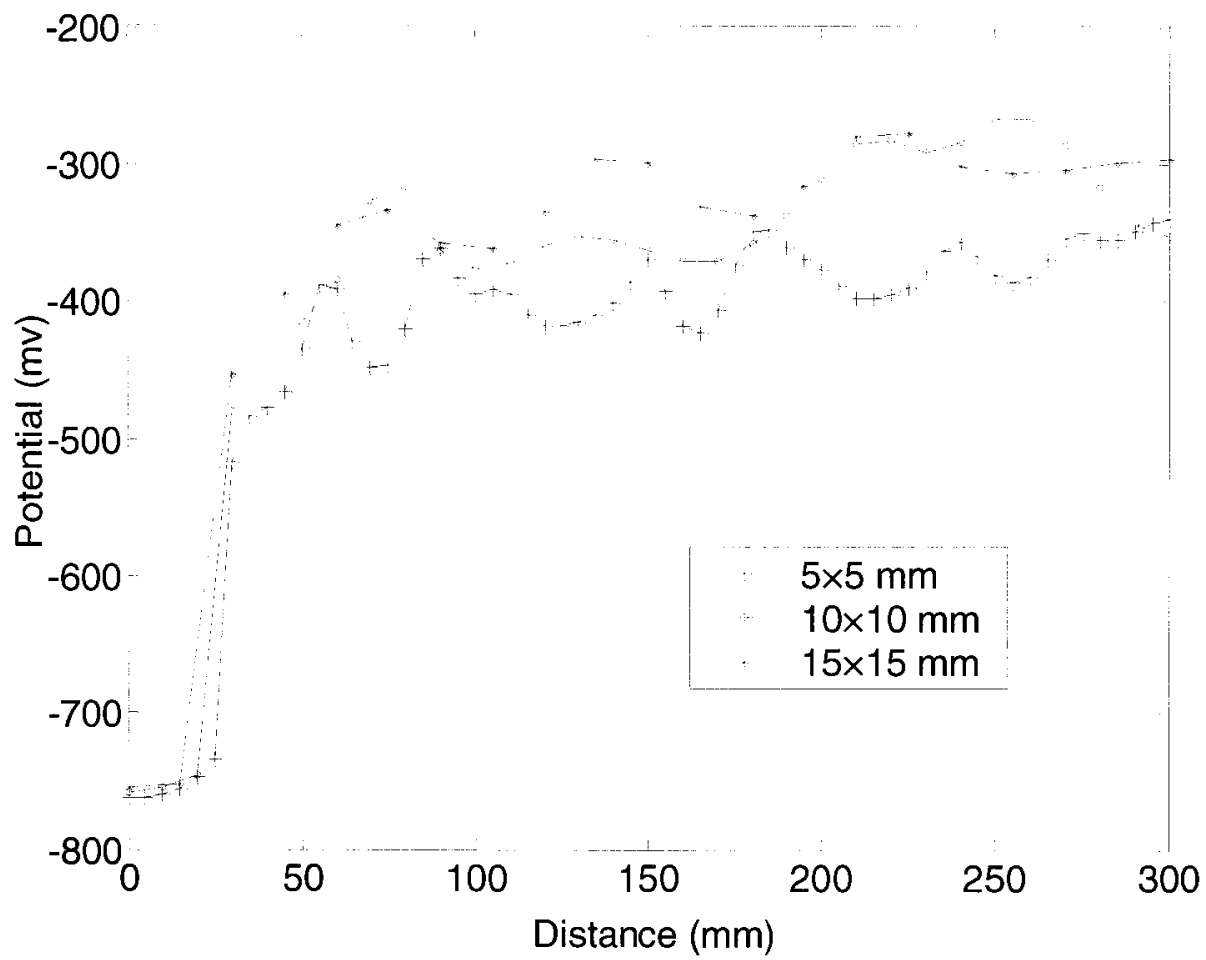

Fig. 5.2: Potential distribution calculated using the DIM (Element size analysis)

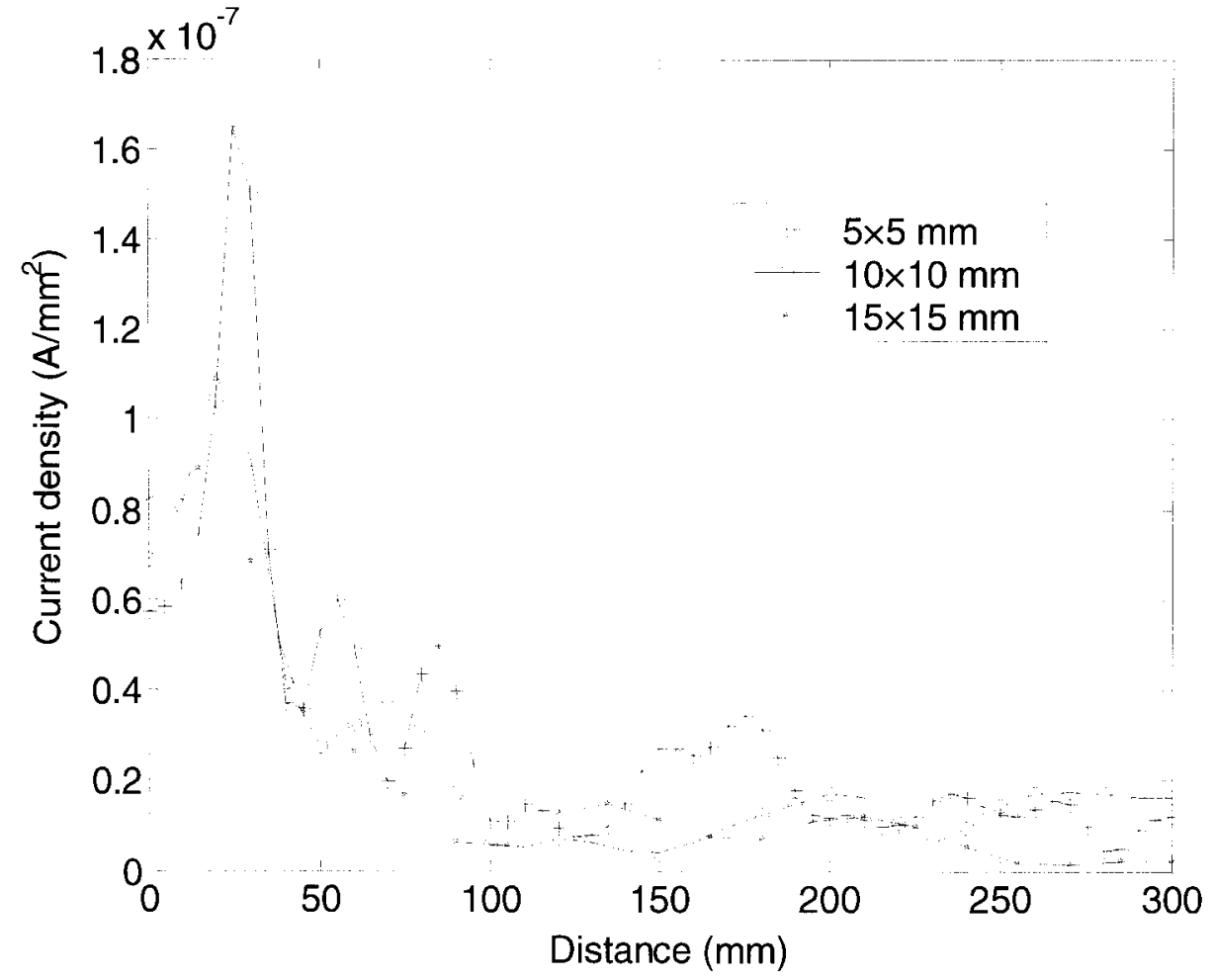

Fig. 5.3: Current density calculated using the DIM (Element size analysis) 


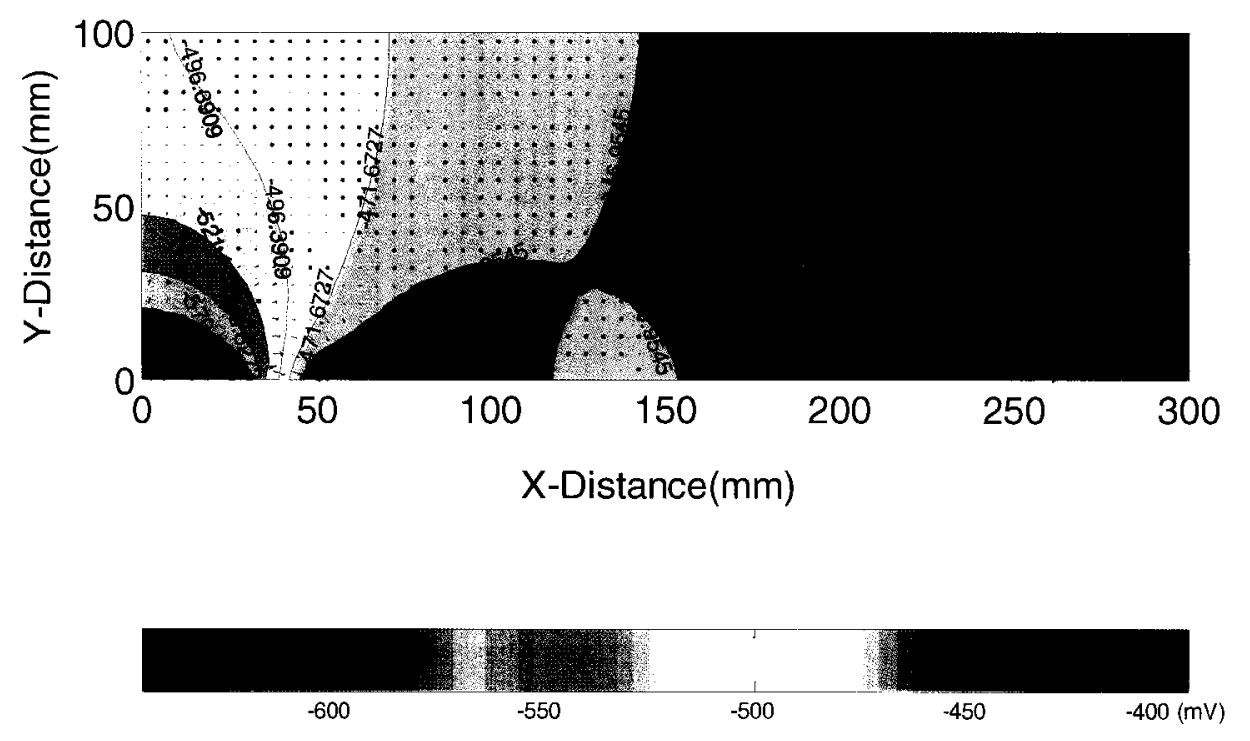

Fig. 5.4: Potential distribution in the domain calculated using the DIM (Element size analysis)

\subsubsection{Effect of Anode-to-cathode ratio}

To investigate the effect of $\mathrm{A} / \mathrm{C}$ ratio on the solution with the DIM, the base case is run for eight cases, whose data is presented in Table 5.2. As it can be seen from this table, the smallest values of the maximum error after 5000 iterations are found to be very large. Figures 5.5 and 5.6 respectively illustrate the potential distribution and current density on the steel surface for the iterations corresponding to the smallest values of the maximum error. It can be observed from these figures that, besides their large maximum error values (i.e. no convergence), there are significant fluctuations in potential distribution and current density, especially along the cathodic regions of the reinforcement. A sample contour plot in Fig. 5.7 for the case for $\mathrm{A} / \mathrm{C}=0.111$ (Case \# 2 in Table 5.2) shows that the distribution of potentials in the domain is not smooth. 
Table 5.2: $\quad \mathrm{A} / \mathrm{C}$ ratio analysis data and results by the DIM

\begin{tabular}{|c|c|c|c|c|c|c|}
\hline Case & $\begin{array}{c}\mathrm{A} / \mathrm{C} \\
\text { ratio }\end{array}$ & $\begin{array}{c}\text { Element } \\
\text { size }(\mathbf{m m})\end{array}$ & $\begin{array}{l}\text { Anode } \\
\text { length } \\
(\mathbf{m m})\end{array}$ & $\begin{array}{l}\text { Max. } \\
\text { error }^{(1)} \\
(\mathbf{m V})\end{array}$ & $\begin{array}{l}\text { Corresponding } \\
\text { iteration }^{(2)}\end{array}$ & $\begin{array}{l}\text { Corresponding } \\
\text { position }^{(3)}(\mathrm{mm})\end{array}$ \\
\hline 1 & 0.034 & 5 & 10 & 64.1 & 419 & 40 \\
\hline 2 & 0.111 & 5 & 30 & 71.7 & 1440 & 90 \\
\hline 3 & 0.2 & 5 & 50 & 67.6 & 3726 & 190 \\
\hline 4 & 0.304 & 5 & 70 & 70.2 & 472 & 110 \\
\hline 5 & 0.429 & 5 & 90 & 67.4 & 4438 & 160 \\
\hline 6 & 0.579 & 5 & 110 & 67.5 & 664 & 180 \\
\hline 7 & 0.765 & 5 & 130 & 71.3 & 5 & 170 \\
\hline 8 & 1 & 5 & 150 & 30.9 & 7 & 180 \\
\hline
\end{tabular}

(1) Smallest value of the maximum error after 5000 iterations

(2) The iteration corresponding to the smallest value of the maximum error

(3) The location corresponding to the smallest value of the maximum error

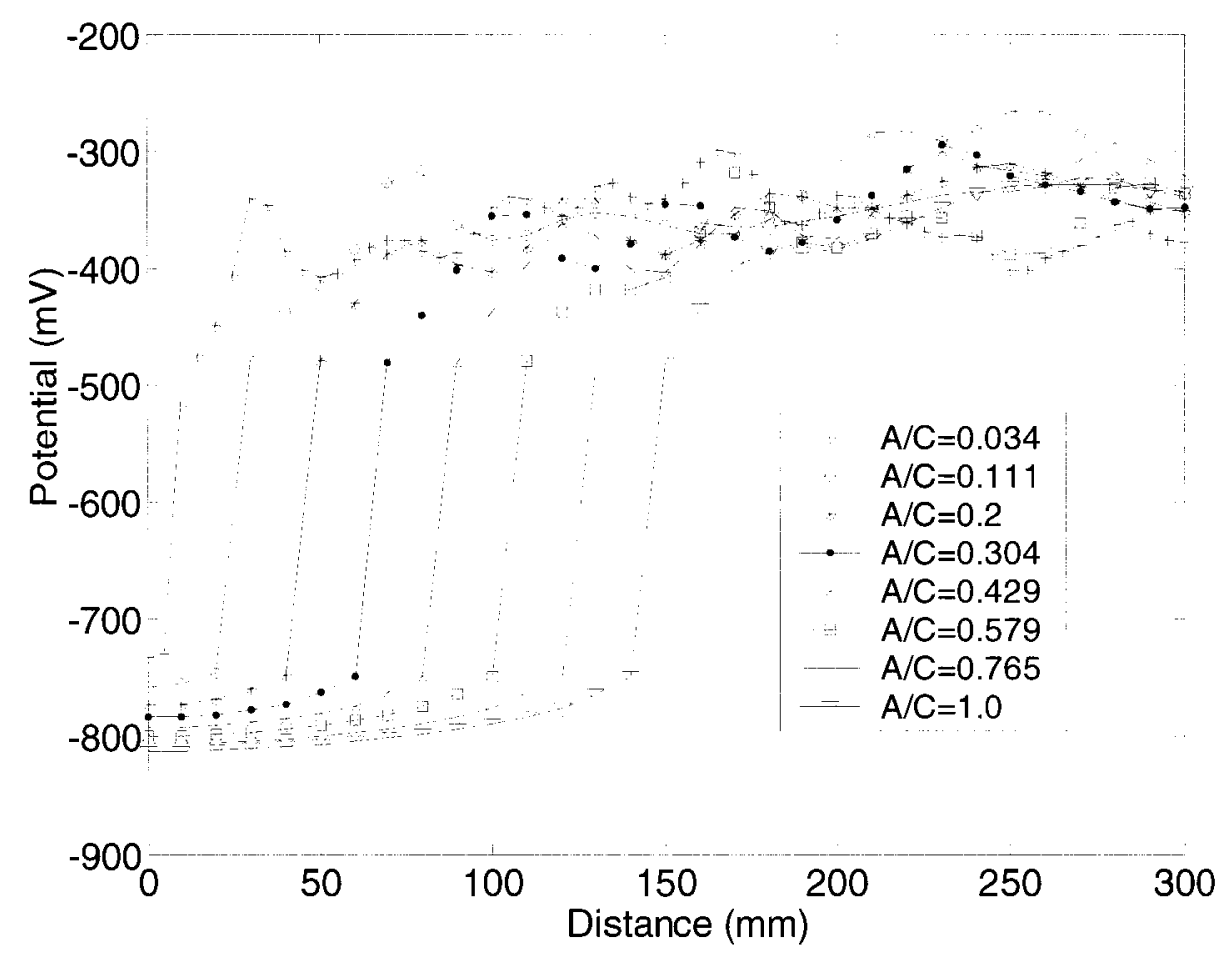

Fig. 5.5: Potential distribution calculated using the DIM (A/C analysis) 


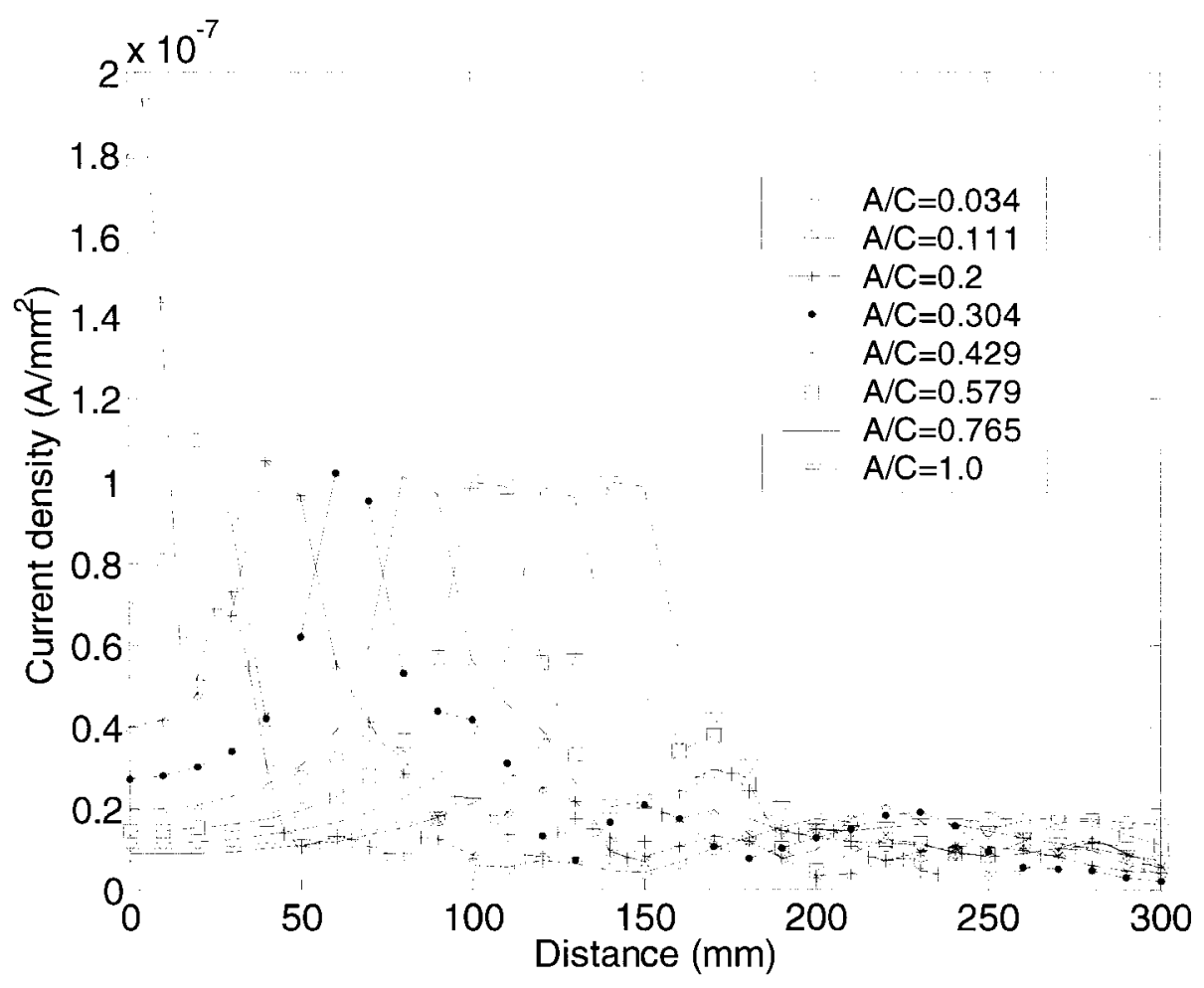

Fig. 5.6: Current density calculated using the DIM (A/C analysis)

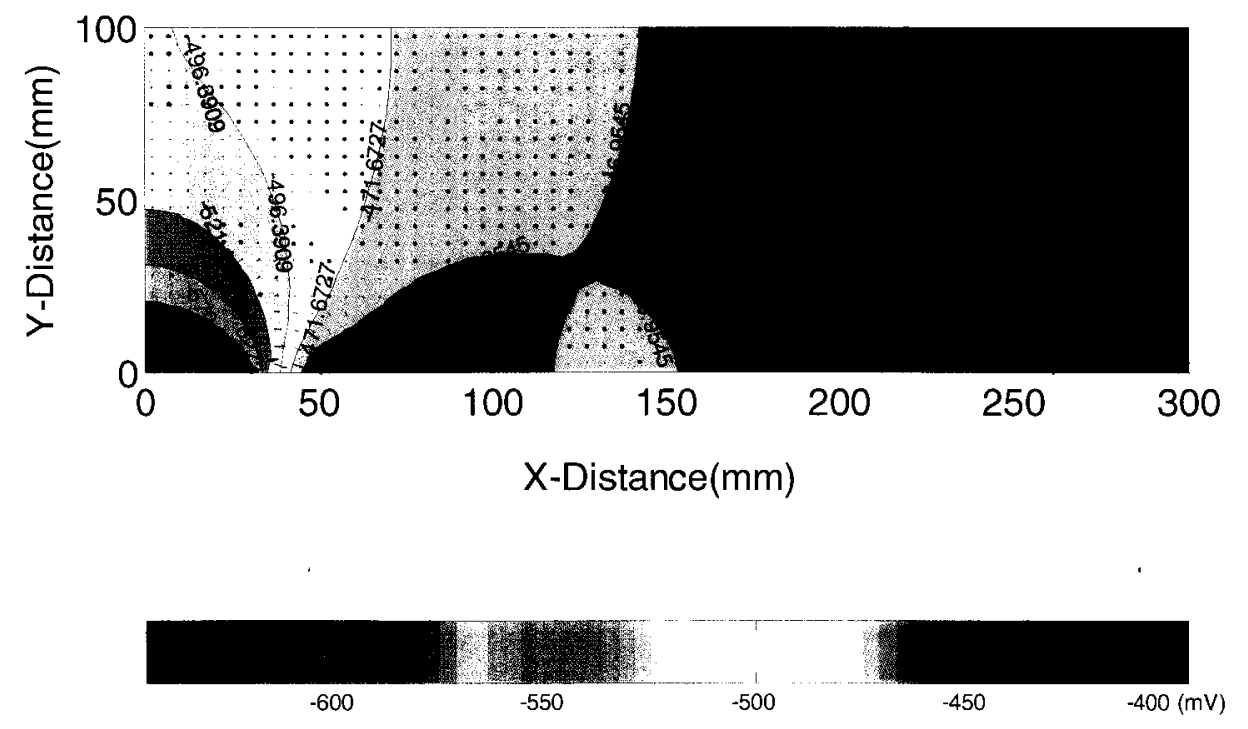

Fig. 5.7: Potential distribution in the domain calculated using the $\mathrm{DIM}(\mathrm{A} / \mathrm{C}=0.111)$ 


\subsubsection{Effect of resistivity}

To investigate the effect of concrete resistivity on the solution with the DIM, the base case is run for seven cases using 5-mm elements, whose data is presented in Table 5.3. As it can be seen from this table, the smallest values of the maximum error after 5000 iterations are found to be very large. Figures 5.8 and 5.9 respectively illustrate the potential distribution and current density on the steel surface for the iterations corresponding to the smallest values of the maximum error. It can be observed from these figures that, besides their large maximum error values (i.e. no convergence), there are significant fluctuations in potential distribution and current density, especially along the cathodic regions of the reinforcement. A sample contour plot in Fig. 5.10 for the case for $r$ $=10,000 \mathrm{ohm}-\mathrm{mm}$ (Case \# 1 in Table 5.3) shows that the distribution of potentials in the domain is not smooth.

Table 5.3: Resistivity analysis data and results by the DIM

\begin{tabular}{|c|c|c|c|c|}
\hline Case & $\begin{array}{c}\text { Resistivity } \\
(\mathbf{o h m}-\mathbf{m m})\end{array}$ & A/C ratio & $\begin{array}{c}\text { Max. error } \\
(\mathbf{m V})\end{array}$ & $\begin{array}{c}\text { Corresponding }_{(\mathbf{1})} \\
\text { iteration }^{(2)}\end{array}$ \\
\hline 1 & $1.00 \mathrm{E}+04$ & 0.111 & $>100$ & N/A \\
\hline 2 & $1.00 \mathrm{E}+05$ & 0.111 & 83.4 & 548 \\
\hline 3 & $3.00 \mathrm{E}+05$ & 0.111 & 86.9 & 1108 \\
\hline 4 & $5.00 \mathrm{E}+05$ & 0.111 & 89 & 3021 \\
\hline 5 & $7.00 \mathrm{E}+05$ & 0.111 & 86.3 & 163 \\
\hline 6 & $9.00 \mathrm{E}+05$ & 0.111 & 82.4 & 4 \\
\hline 7 & $1.00 \mathrm{E}+06$ & 0.111 & 85.9 & 466 \\
\hline
\end{tabular}

(1) Smallest value of the maximum error after 5000 iterations

(2) The iteration corresponding to the smallest value of the maximum error 


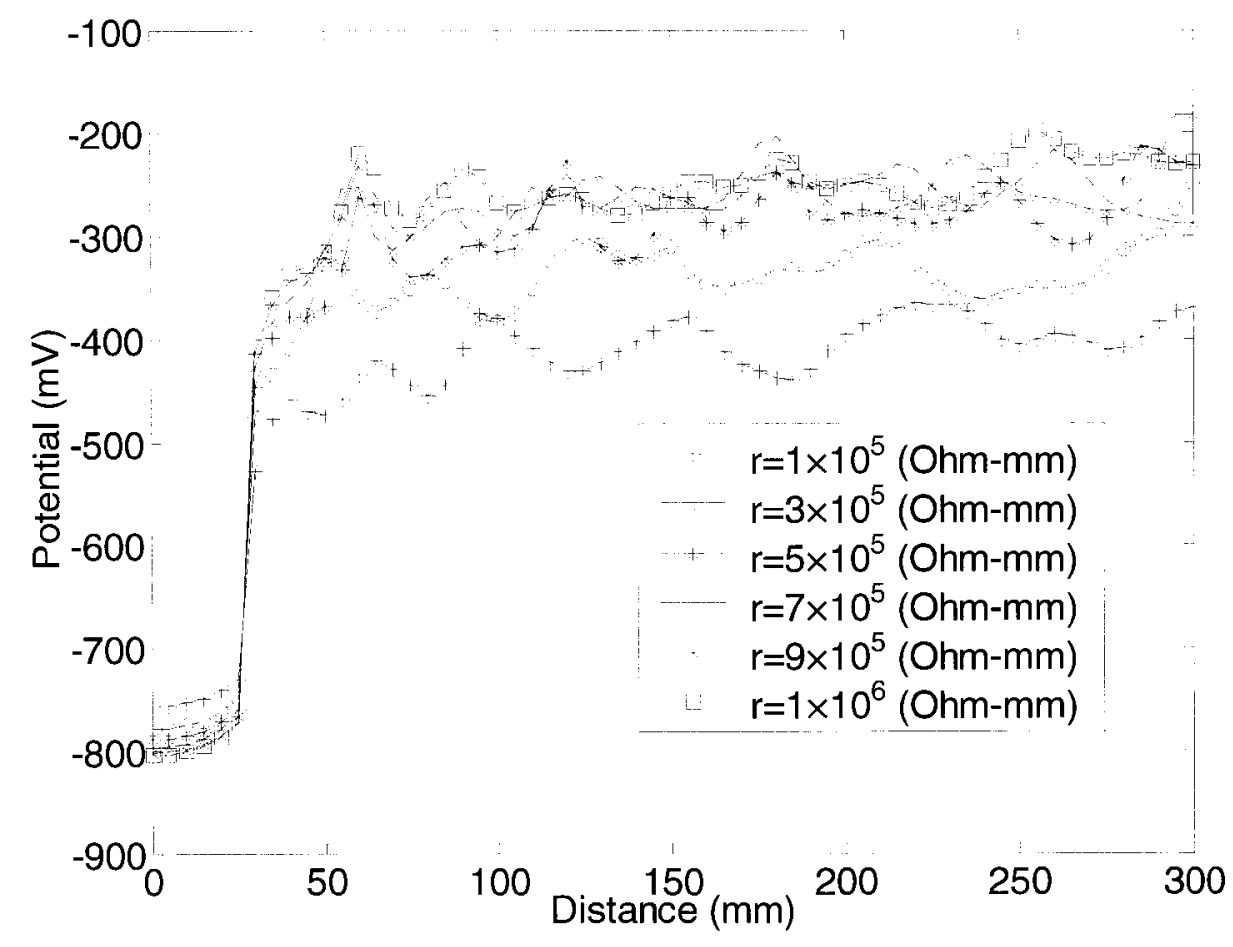

Fig. 5.8: Potential distribution calculated using the DIM (Resistivity analysis)

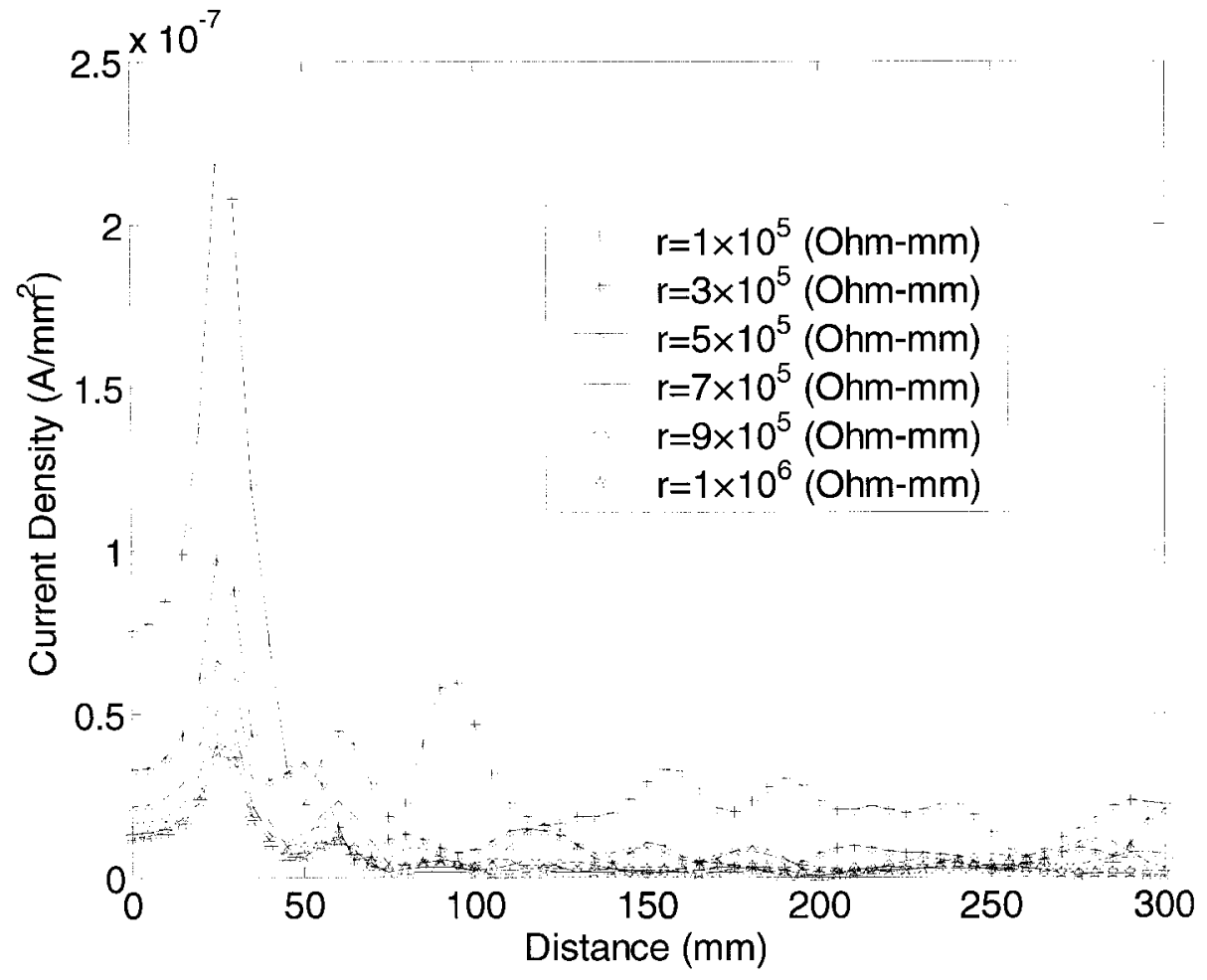

Fig. 5.9: Current density calculated using the DIM (Resistivity analysis) 

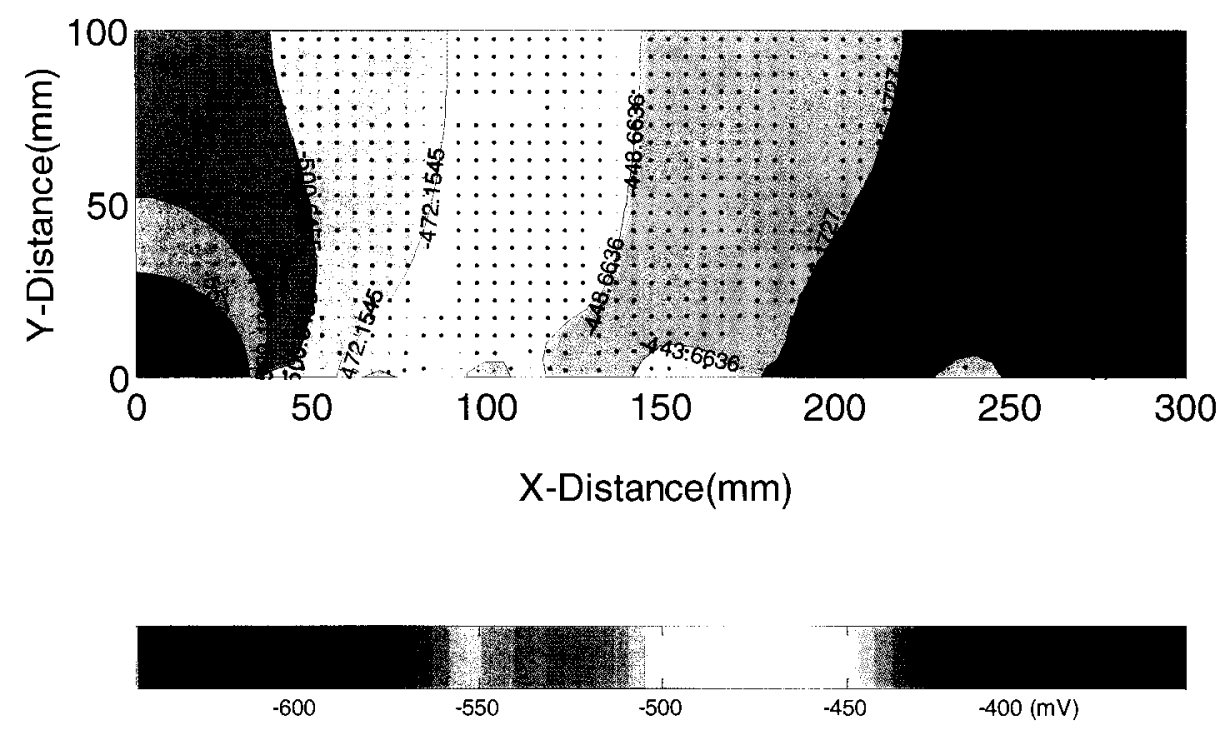

Fig. 5.10: Potential distribution in the domain calculated using the DIM ( $r=10,000$ ohm-mm)

\subsection{Solution by modified direct iteration method (MDIM)}

In order to demonstrate the application of the MDIM, the base case, whose data is provided in Table 5.1, is run to investigate the effects of the following parameters on the numerical solution: (1) element size, (2) A/C ratio; (3) resistivity of concrete. Before the analysis, studies on the selection of the under-relaxation and limiting current density selection factors and on the effect of initial conditions $\left(i_{a}{ }^{0}\right.$ and $\left.i_{c}{ }^{0}\right)$ are carried out.

\subsubsection{Under-relaxation factor}

In order to determine the effect of different under-relaxation factors on the numerical solution of the corrosion problem, a study is carried out by varying $\xi$ from 0.003 to 0.5 and using three limiting current densities at $1 \times 10^{-6}, 1 \times 10^{-7}, 1 \times 10^{-8} \mathrm{~A} / \mathrm{mm}^{2}$ as shown in Table 5.4. 
From Figs. 5.11 and 5.12, it can be observed that the potential distribution and current density along the reinforcement do not change with varying $\xi$ values from 0.003 to 0.1 when the limiting current density is large (i.e. $1 \times 10^{-6} \mathrm{~A} / \mathrm{mm}^{2}$ ). Whereas when $\xi=0.5$ the potential distribution fluctuates and the maximum error rises up to $30.6 \mathrm{mV}$. As illustrated in Fig. 5.13, for larger $\xi$ values, the initial error is large, but the convergence rate is faster (i.e. iteration \# less than 200). When $\xi=0.5$, convergence is not stable.

When the limiting current density is $10^{-7} \mathrm{~A} / \mathrm{mm}^{2}$, the potential distribution and current density have a good performance for all $\xi$ values except for the case (Case \# 11) which has $\xi=0.1$ as shown in Figs. 5.14 and 5.15. For this case, although the maximum error is low, we observe fluctuations in potential and current density towards the far end of the cathodic region. Once again, it can be observed from Table 5.4 and Fig. 5.16, the convergence rate becomes faster as the under-relaxation factor increases.

For low limiting current densities (i.e. $10^{-8} \mathrm{~A} / \mathrm{mm}^{2}$ ), Case \# 13 has the most accurate results after the least number of iterations. In addition, as illustrated in Figs. 5.17 and 5.18, in cases with under-relaxation factor greater than 0.008 , we observe fluctuations in potential and current density.

After these observations, it is decided that a common under-relaxation factor for all levels of limiting current density can be used. This value should be as large as possible for achieving fast convergence, but small enough to avoid any numerical issues and 
fluctuations in potential and current density predictions. Thus in the numerical investigation presented in this chapter, the under-relaxation factor is selected as 0.003 .

Table 5.4: Data and results for under-relaxation factor analysis

\begin{tabular}{|c|c|c|c|c|c|c|c|c|}
\hline Case & $\begin{array}{c}\text { Element } \\
\text { size } \\
(\mathrm{mm})\end{array}$ & $\left(\begin{array}{c}r \\
(o h m-m m)\end{array}\right.$ & $\mathrm{A} / \mathrm{C}$ & $i_{L}\left(\mathrm{~A} / \mathrm{mm}^{2}\right)$ & $\xi$ & $\Psi$ & $\begin{array}{c}\text { Max. error } \\
(\mathrm{mV})\end{array}$ & $\begin{array}{c}\text { Corresponding } \\
\text { iteration }^{(2)}\end{array}$ \\
\hline 1 & 5 & 140000 & 0.111 & $10^{-6}$ & 0.003 & N/A & $<0.1$ & 1189 \\
\hline 2 & 5 & 140000 & 0.111 & $10^{-6}$ & 0.005 & N/A & $<0.1$ & 842 \\
\hline 3 & 5 & 140000 & 0.111 & $10^{-6}$ & 0.007 & N/A & $<0.1$ & 658 \\
\hline 4 & 5 & 140000 & 0.111 & $10^{-6}$ & 0.009 & N/A & $<0.1$ & 545 \\
\hline 5 & 5 & 140000 & 0.111 & $10^{-6}$ & 0.05 & N/A & $<0.1$ & 594 \\
\hline 6 & 5 & 140000 & 0.111 & $10^{-6}$ & 0.1 & N/A & 0.1 & 226 \\
\hline 7 & 5 & 140000 & 0.111 & $10^{-6}$ & 0.5 & N/A & 30.6 & 2018 \\
\hline 8 & 5 & 140000 & 0.111 & $10^{-7}$ & 0.003 & N/A & 0.1 & 1200 \\
\hline 9 & 5 & 140000 & 0.111 & $10^{-7}$ & 0.005 & N/A & $<0.1$ & 838 \\
\hline 10 & 5 & 140000 & 0.111 & $10^{-7}$ & 0.05 & N/A & $<0.1$ & 205 \\
\hline 11 & 5 & 140000 & 0.111 & $10^{-7}$ & 0.1 & N/A & $<0.1$ & 210 \\
\hline 12 & 5 & 140000 & 0.111 & $10^{-8}$ & 0.002 & 0.995 & $<0.1$ & 1565 \\
\hline 13 & 5 & 140000 & 0.111 & $10^{-8}$ & 0.003 & 0.995 & $<0.1$ & 1228 \\
\hline 14 & 5 & 140000 & 0.111 & $10^{-8}$ & 0.004 & 0.995 & $<0.1$ & 4696 \\
\hline 15 & 5 & 140000 & 0.111 & $10^{-8}$ & 0.005 & 0.995 & 0.2 & 612 \\
\hline 16 & 5 & 140000 & 0.111 & $10^{-8}$ & 0.006 & 0.995 & 0.2 & 802 \\
\hline 17 & 5 & 140000 & 0.111 & $10^{-8}$ & 0.008 & 0.995 & 0.2 & 1640 \\
\hline 18 & 5 & 140000 & 0.111 & $10^{-8}$ & 0.05 & 0.995 & 2.1 & 4254 \\
\hline 19 & 5 & 140000 & 0.111 & $10^{-8}$ & 0.1 & 0.995 & 4.4 & 1728 \\
\hline
\end{tabular}

(1) Smallest value of the maximum error after 5000 iterations

(2) The iteration corresponding to the smallest value of the maximum error 


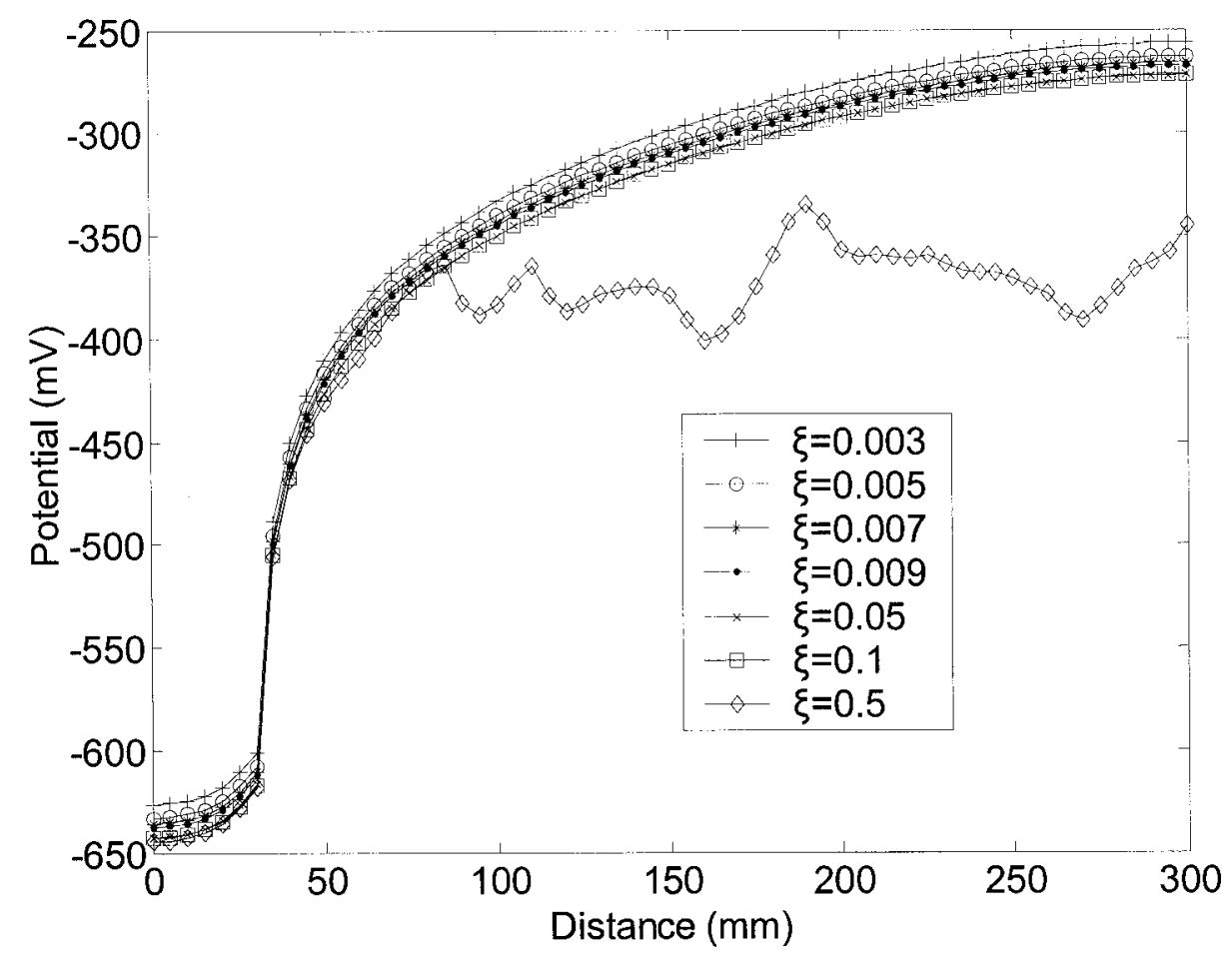

Fig. 5.11: Potential distribution on the rebar for different $\xi$ factors $\left(i_{L}=1 \times 10^{-6} \mathrm{~A} / \mathrm{mm}^{2}\right)$

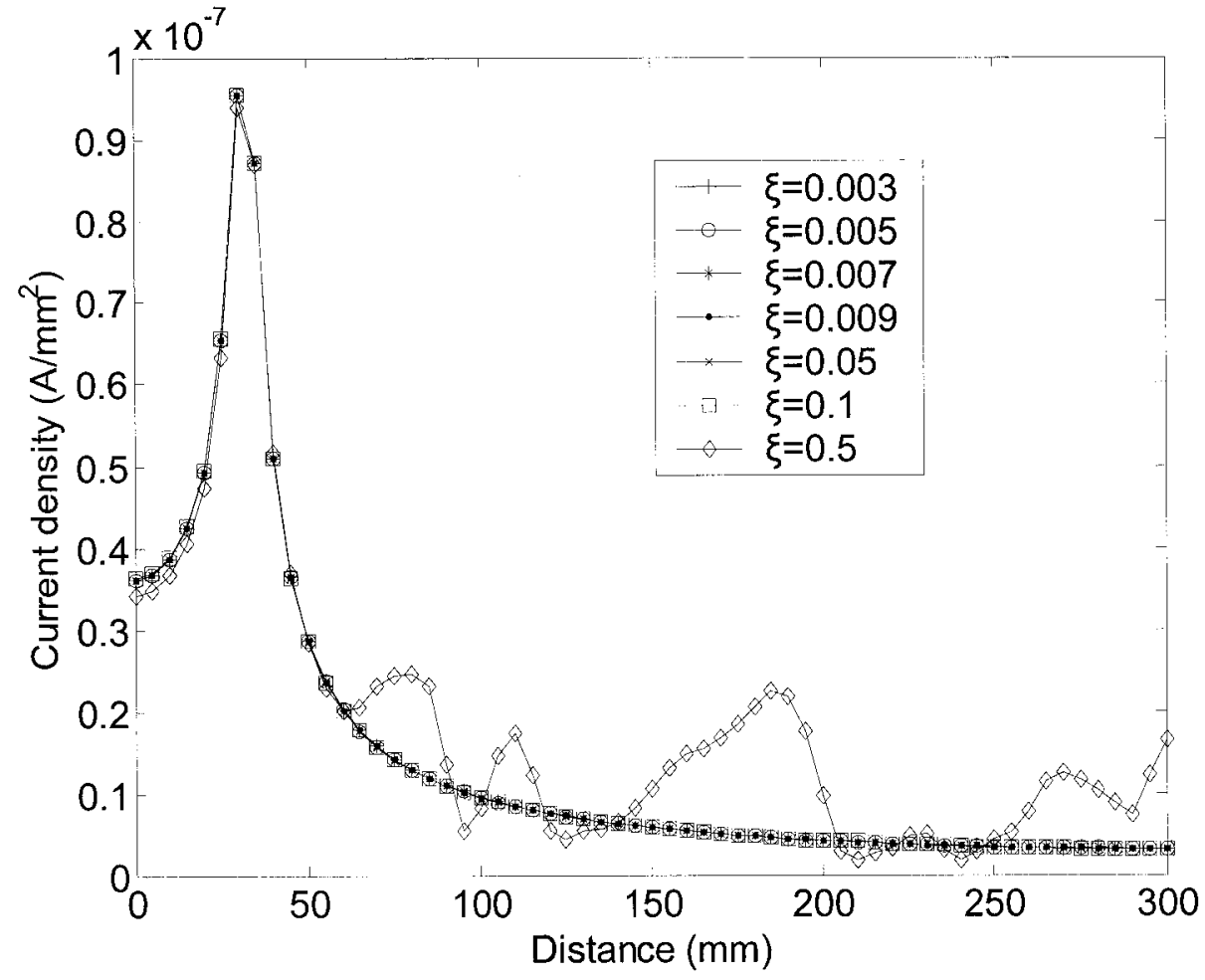

Fig. 5.12: Current density on the rebar for different $\xi$ factors $\left(i_{L}=1 \times 10^{-6} \mathrm{~A} / \mathrm{mm}^{2}\right)$ 


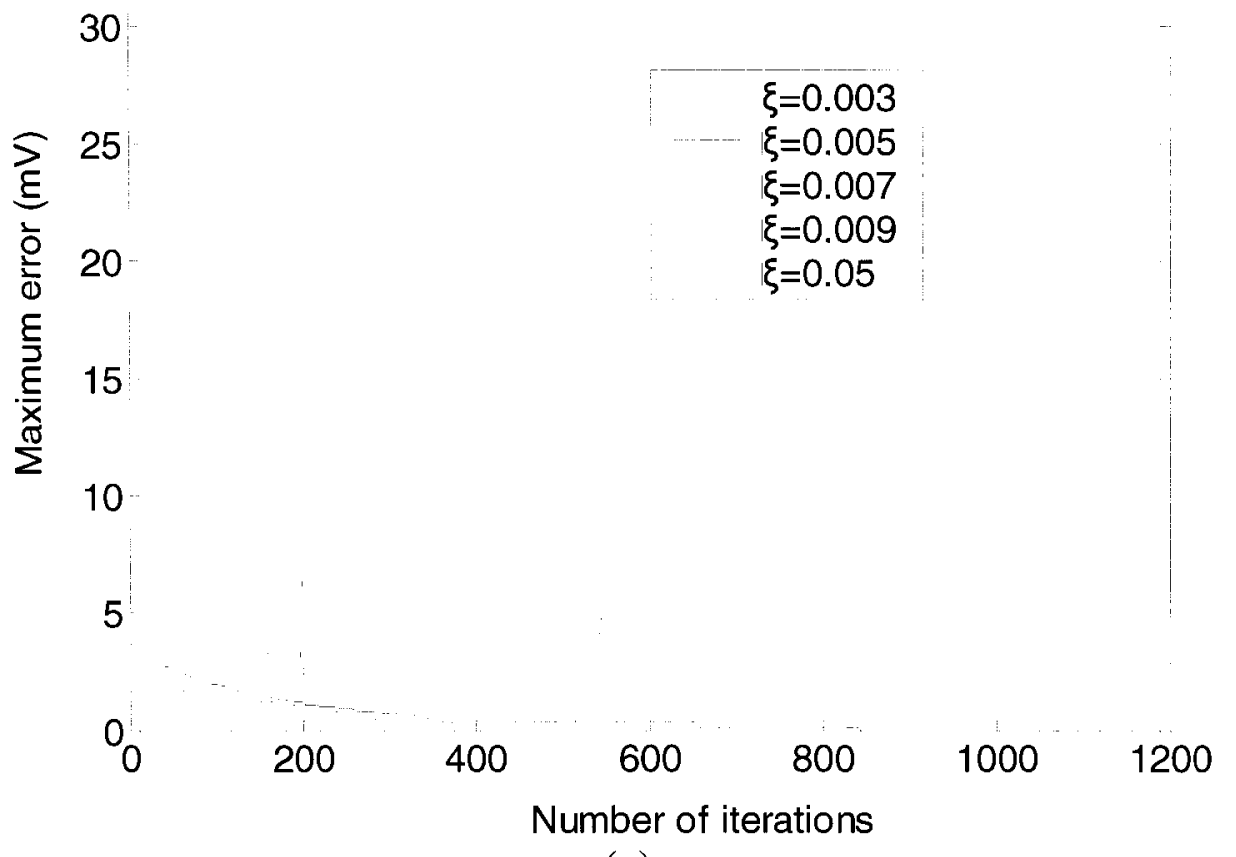

(a)

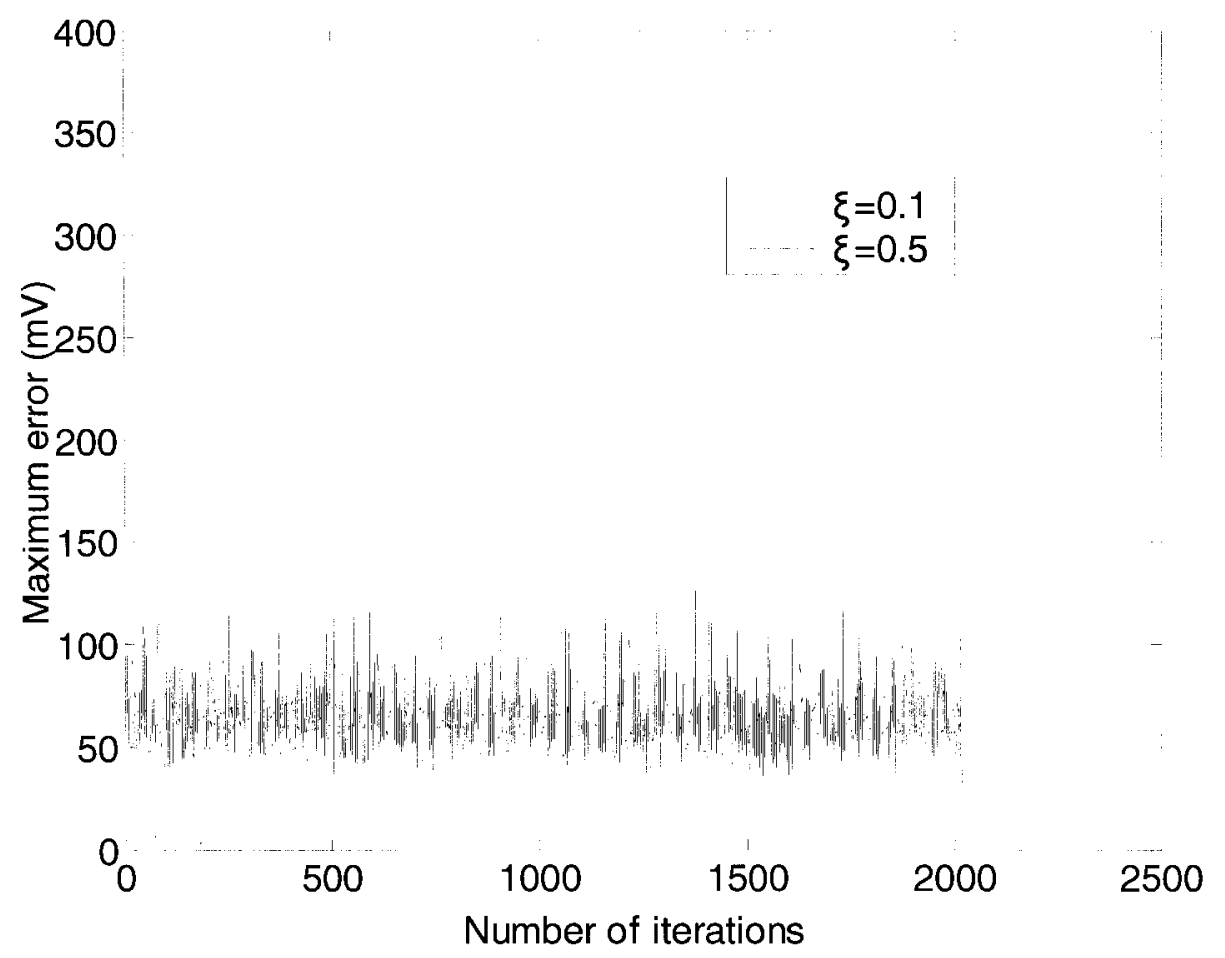

(b)

Fig. 5.13: Convergence process for different under-relaxation factors when $i_{L}=1 \times 10^{-6}$ $\mathrm{A} / \mathrm{mm}^{2}$ (a) $\xi=0.003-0.05$ (b) $\xi=0.1-0.5$ 


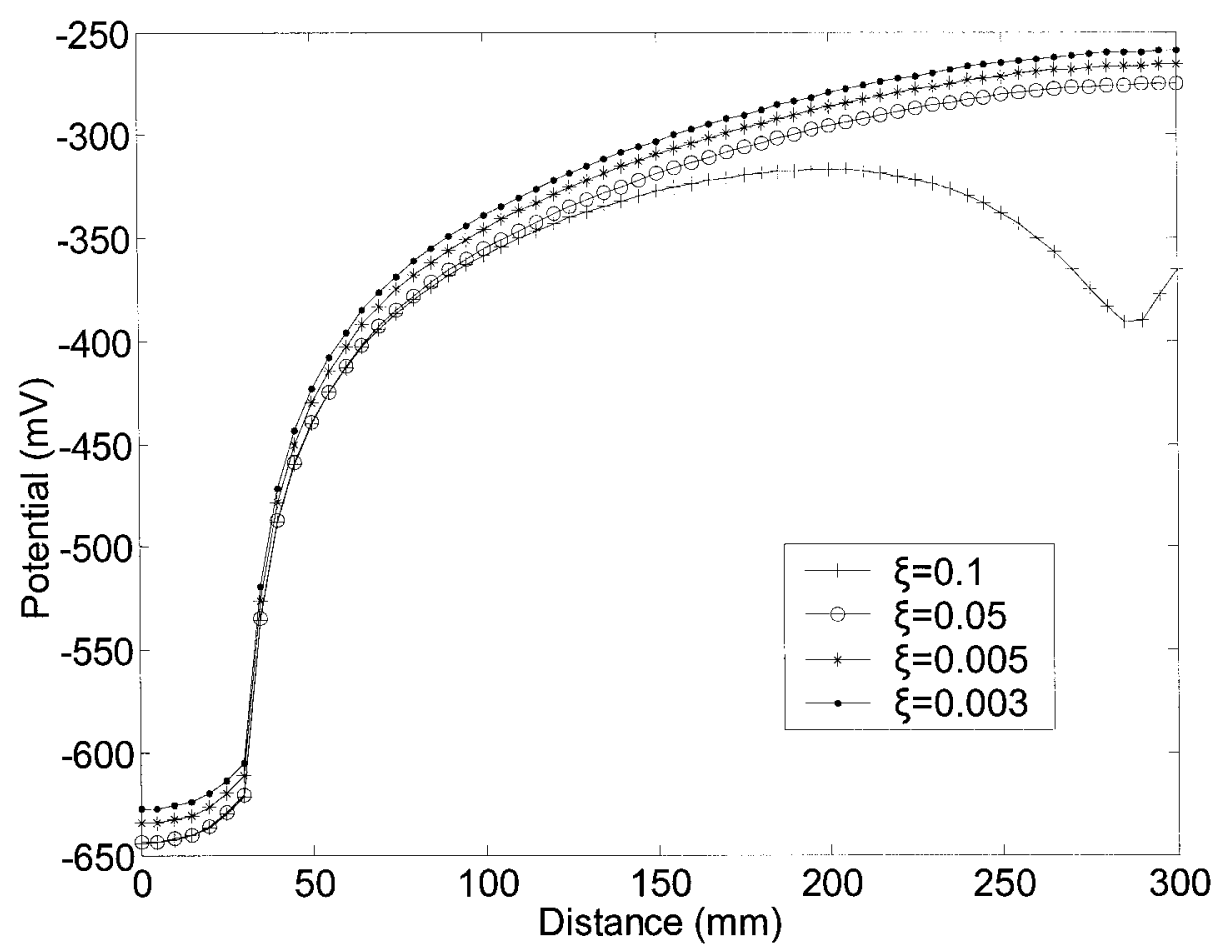

Fig. 5.14: Potential distribution on the rebar for different $\xi$ factors $\left(i_{L}=1 \times 10^{-7} \mathrm{~A} / \mathrm{mm}^{2}\right)$

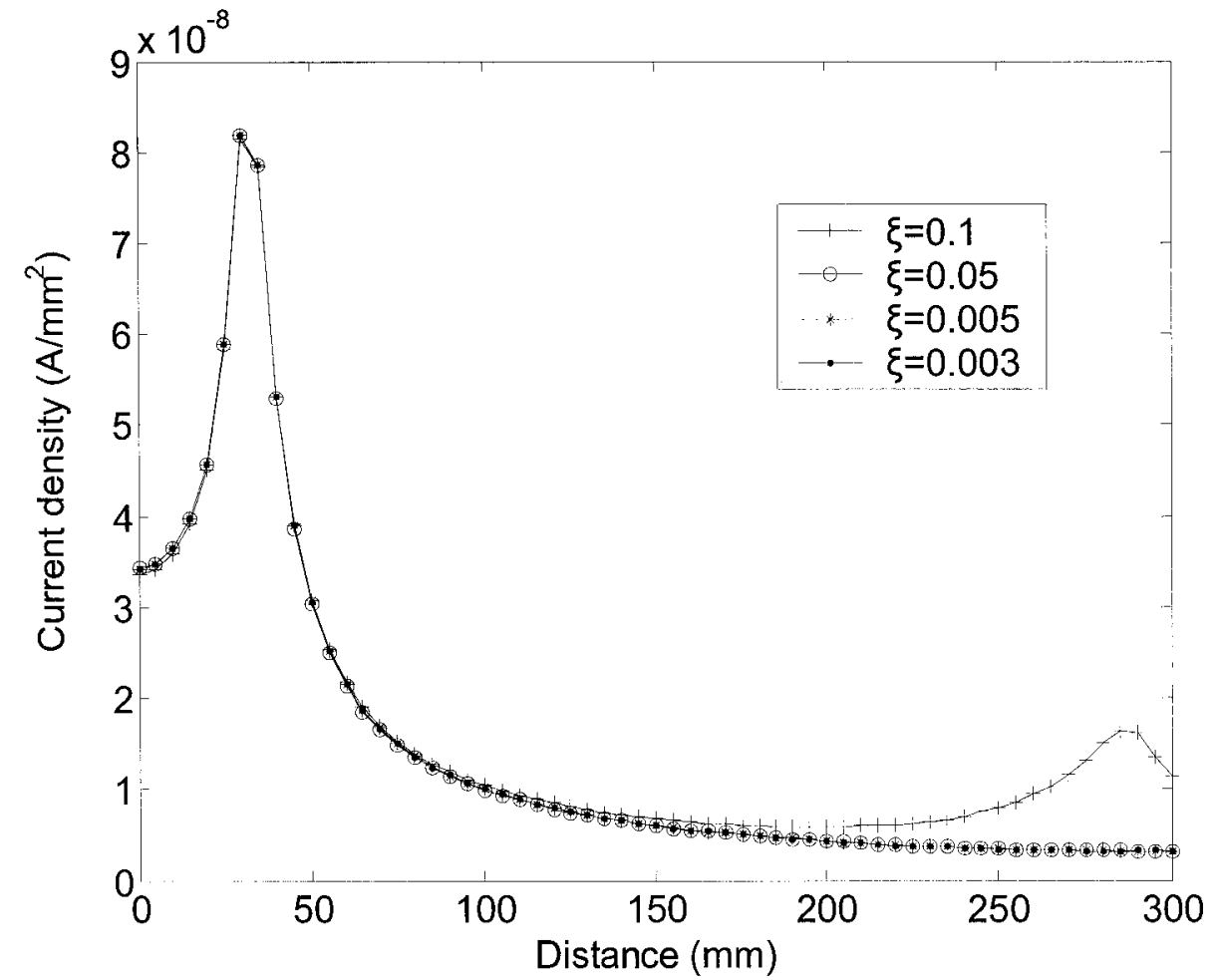

Fig. 5.15: Current density on the rebar for different $\xi$ factors $\left(i_{L}=1 \times 10^{-7} \mathrm{~A} / \mathrm{mm}^{2}\right)$ 
60

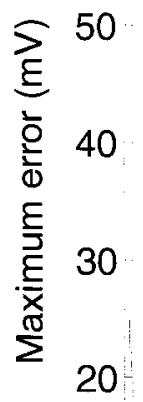

$$
\begin{aligned}
& \xi=0.1 \\
& \xi=0.05
\end{aligned}
$$$$
\xi=0.005
$$$$
\xi=0.003
$$

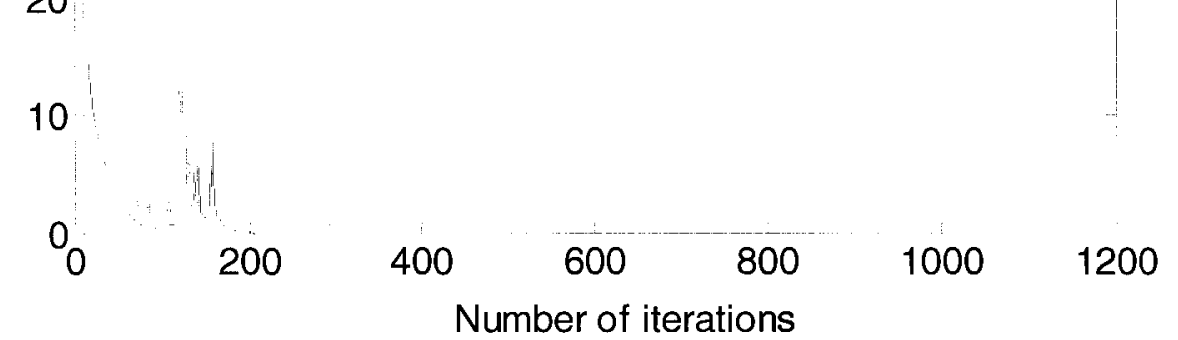

Fig. 5.16: Convergence process for different $\xi$ factors $\left(i_{L}=1 \times 10^{-7} \mathrm{~A} / \mathrm{mm}^{2}\right)$

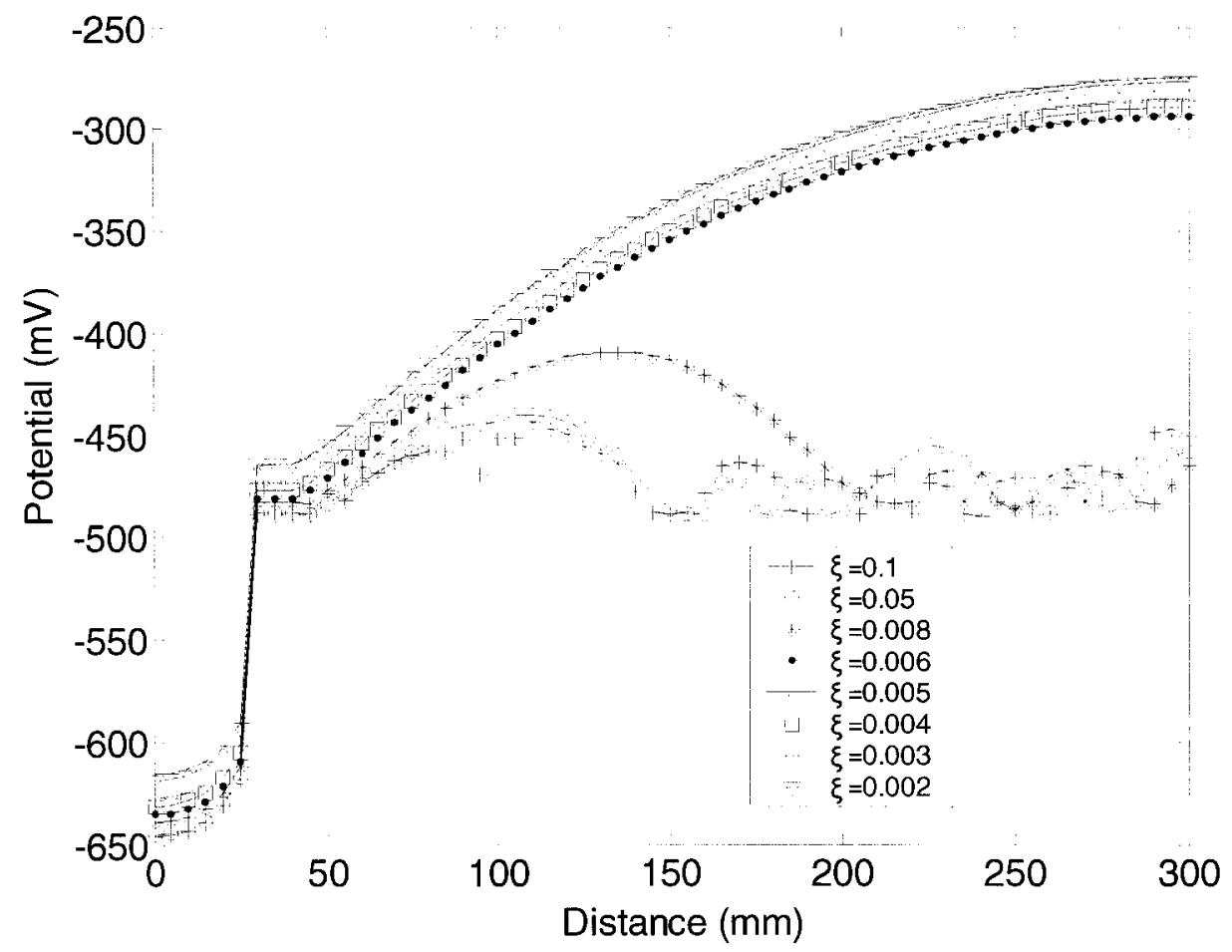

Fig. 5.17: Potential distribution on the rebar for different $\xi$ factors $\left(i_{L}=1 \times 10^{-8}\right.$ $\mathrm{A} / \mathrm{mm}^{2}$ ) 


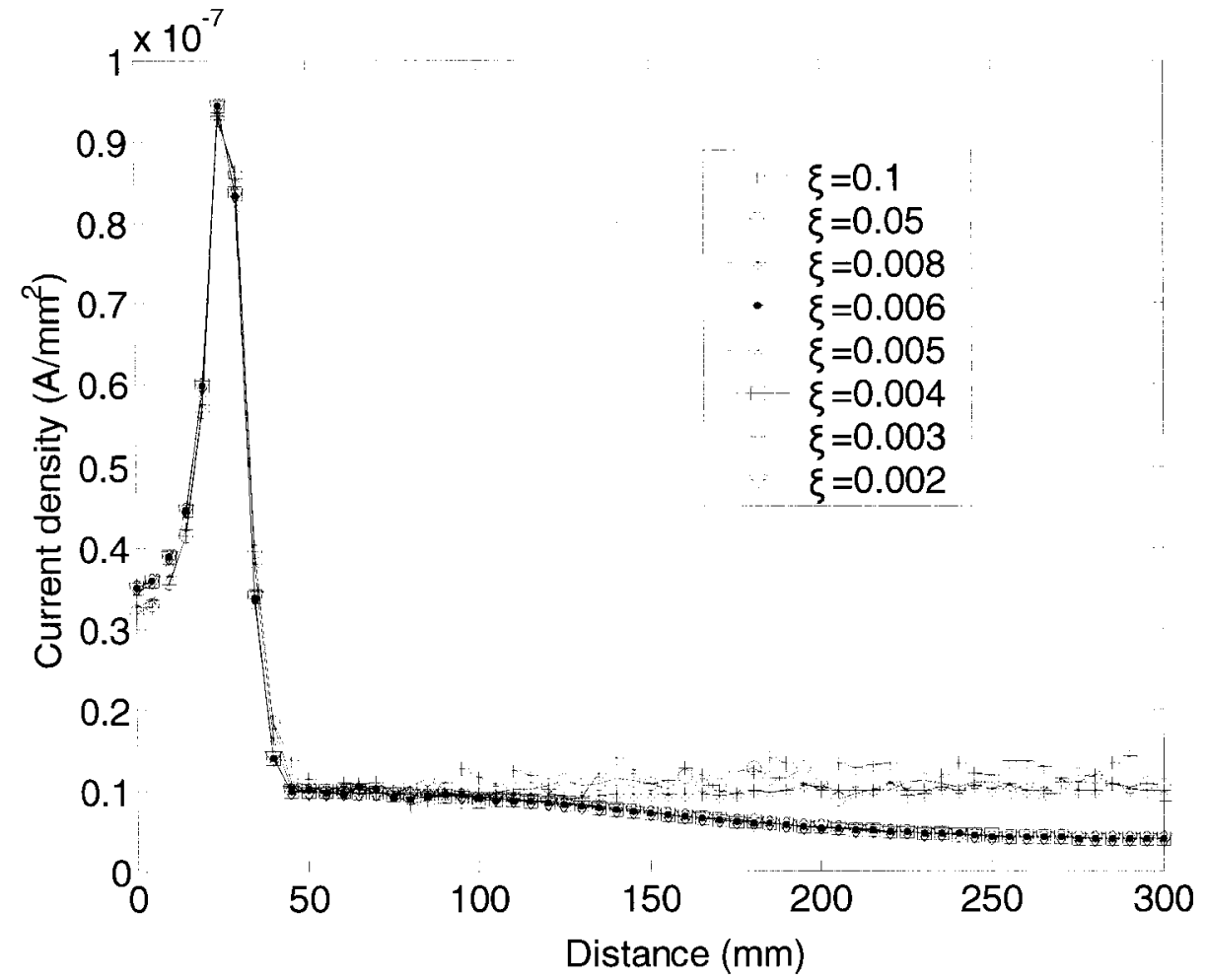

Fig. 5.18: Current density on the rebar for different $\xi$ factors $\left(i_{L}=1 \times 10^{-8} \mathrm{~A} / \mathrm{mm}^{2}\right)$

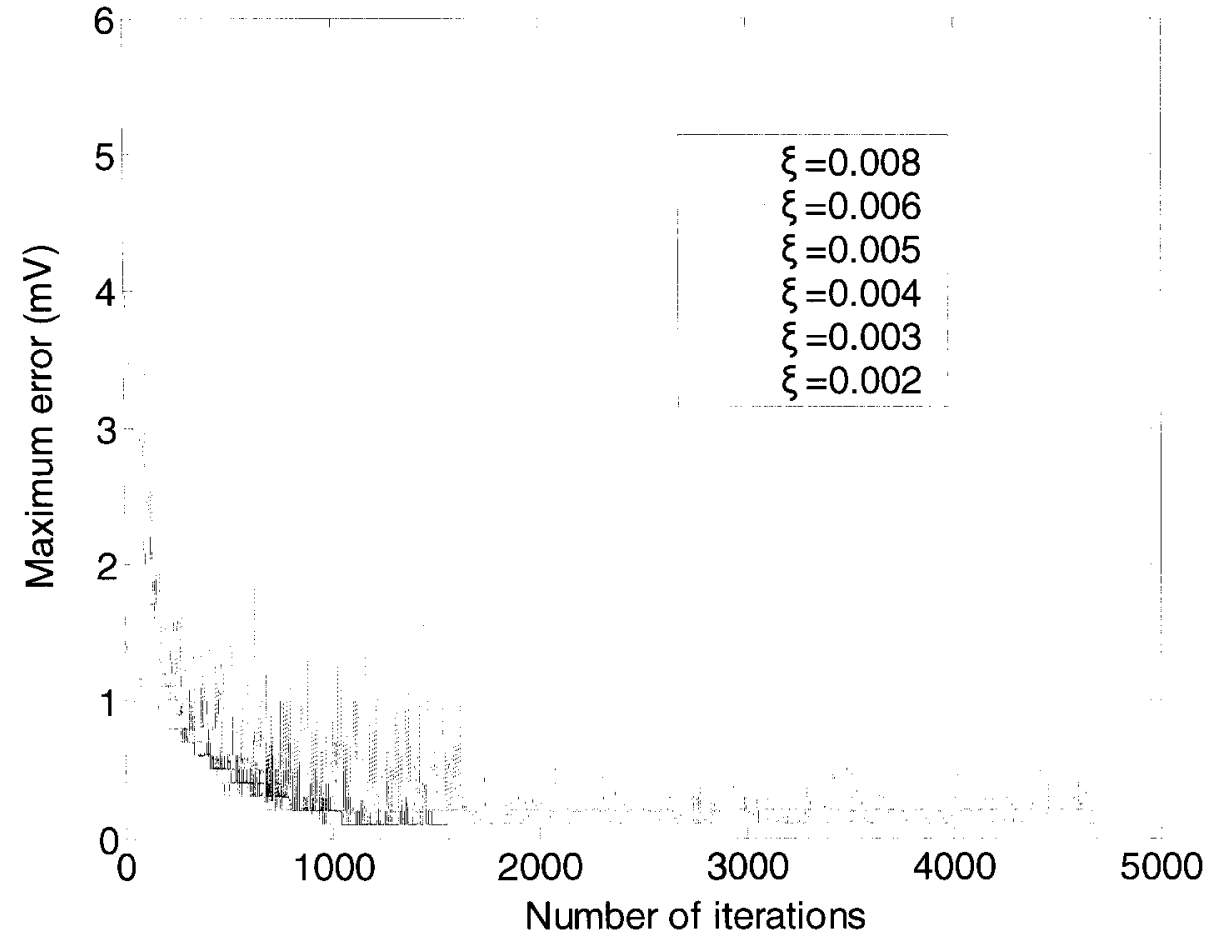

(a) 
60

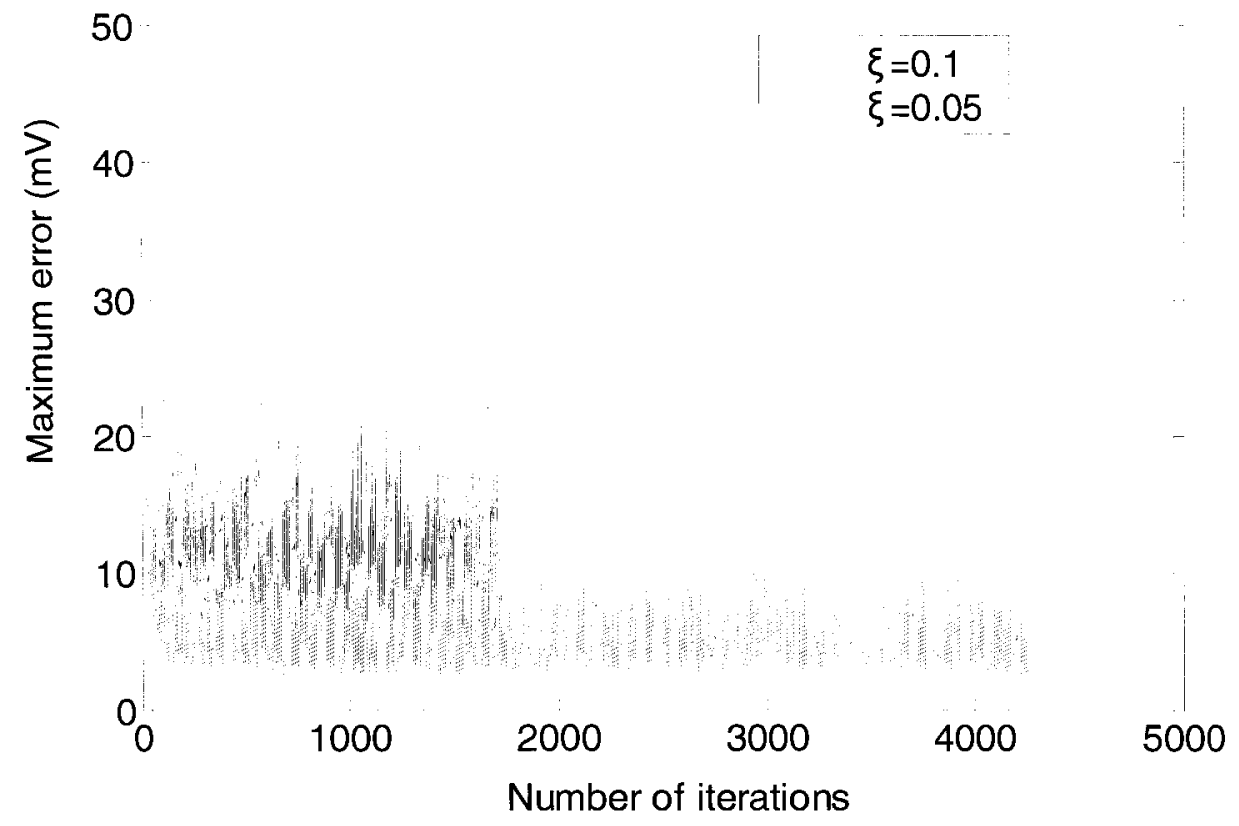

(b)

Fig. 5.19: Convergence process for different under-relaxation factors when $i_{L}=1 \times 10^{-8}$ $\mathrm{A} / \mathrm{mm}^{2}$ (a) $\xi=0.1-0.5$ (b) $\xi=0.003-0.05$

\subsubsection{Initial condition analysis}

As discussed in the previous chapter, non-linear solution algorithms require initial assumptions of current densities on the steel surface. In order to investigate the effect of initial values of the anodic $\left(i_{a}{ }^{0}\right)$ and cathodic $\left(i_{c}{ }^{0}\right)$ current densities on the numerical solution of the corrosion problem, a series of non-zero initial conditions are studied. The initial conditions are chosen from a wide range as shown in Table 5.5, such that corrosion current on the anodic surface is equal to the corrosion current on the cathodic surface (i.e. $\left.I=i_{a} A_{a}=i_{c} A_{c}\right)$. In addition, the initial assumptions must be less than the limiting current density, which is an upper bound for corrosion rate in an electrochemical system. As illustrated in Figs. 5.20-5.22, the initial assumptions for the current densities do not have any impact on the results of the analysis or on the convergence rate. For the numerical 
investigation presented in this chapter, the initial conditions for both cathodic and anodic current densities are selected as $2 \times 10^{-12} \mathrm{~A} / \mathrm{mm}^{2}$, corresponding to Case \#2 in Table 5.5.

Table 5.5: Data and results for initial condition analysis

\begin{tabular}{|c|c|c|c|c|c|c|c|c|c|}
\hline Case & $\begin{array}{c}\text { Element } \\
\text { size } \\
(\mathbf{m m})\end{array}$ & $\begin{array}{c}r \\
(\text { ohm-mm) }\end{array}$ & $\mathbf{A} / \mathbf{C}$ & $\begin{array}{c}i_{L} \\
\left(\mathrm{~A} / \mathbf{m m}^{2}\right)\end{array}$ & $\xi$ & $\begin{array}{c}i_{a}{ }^{0} \\
\left(\mathrm{~A} / \mathbf{m m}^{2}\right)\end{array}$ & $\begin{array}{c}i_{c}{ }^{0} \\
\left(\mathrm{~A} / \mathbf{m m}^{2}\right)\end{array}$ & $\begin{array}{c}\text { Max. } \\
\text { Error }{ }^{(1)} \\
(\mathbf{m V})\end{array}$ & $\begin{array}{c}\text { Corresponding } \\
\text { iteration }^{(2)}\end{array}$ \\
\hline 1 & 5 & 140000 & 0.111 & $10^{-6}$ & 0.003 & $9 \times 10^{-12}$ & $1 \times 10^{-12}$ & $<0.1$ & 1187 \\
\hline 2 & 5 & 140000 & 0.111 & $10^{-6}$ & 0.003 & $2 \times 10^{-12}$ & $2 \times 10^{-12}$ & $<0.1$ & 1189 \\
\hline 3 & 5 & 140000 & 0.111 & $10^{-6}$ & 0.003 & $4.5 \times 10^{-11}$ & $5 \times 10^{-12}$ & $<0.1$ & 1180 \\
\hline 4 & 5 & 140000 & 0.111 & $10^{-6}$ & 0.003 & $4.5 \times 10^{-10}$ & $5 \times 10^{-11}$ & $<0.1$ & 1191 \\
\hline
\end{tabular}

(1) Smallest value of the maximum error after 5000 iterations

(2) The iteration corresponding to the smallest value of the maximum error

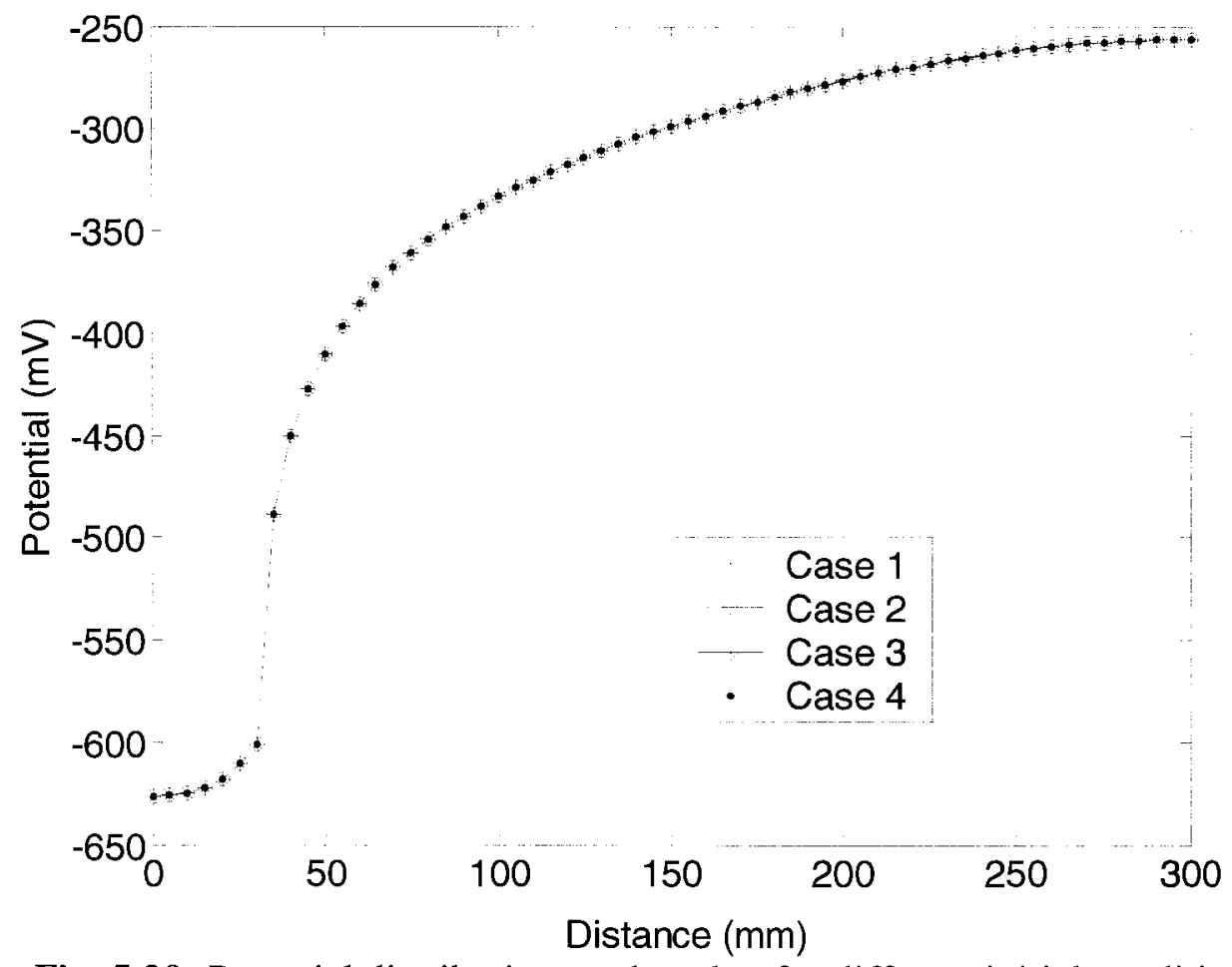

Fig. 5.20: Potential distribution on the rebar for different initial conditions 


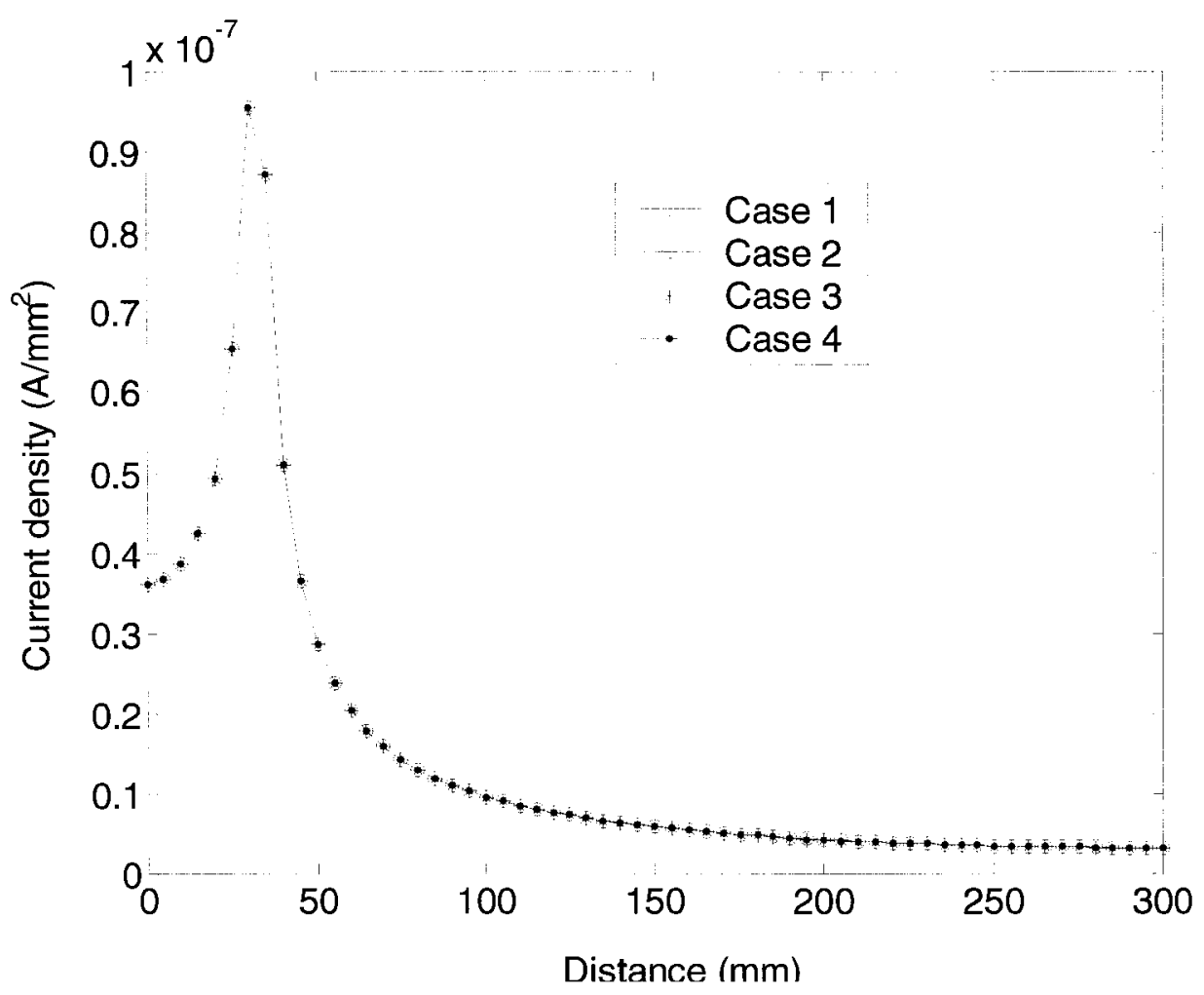

Fig. 5.21: Current density distribution on the rebar for different initial conditions

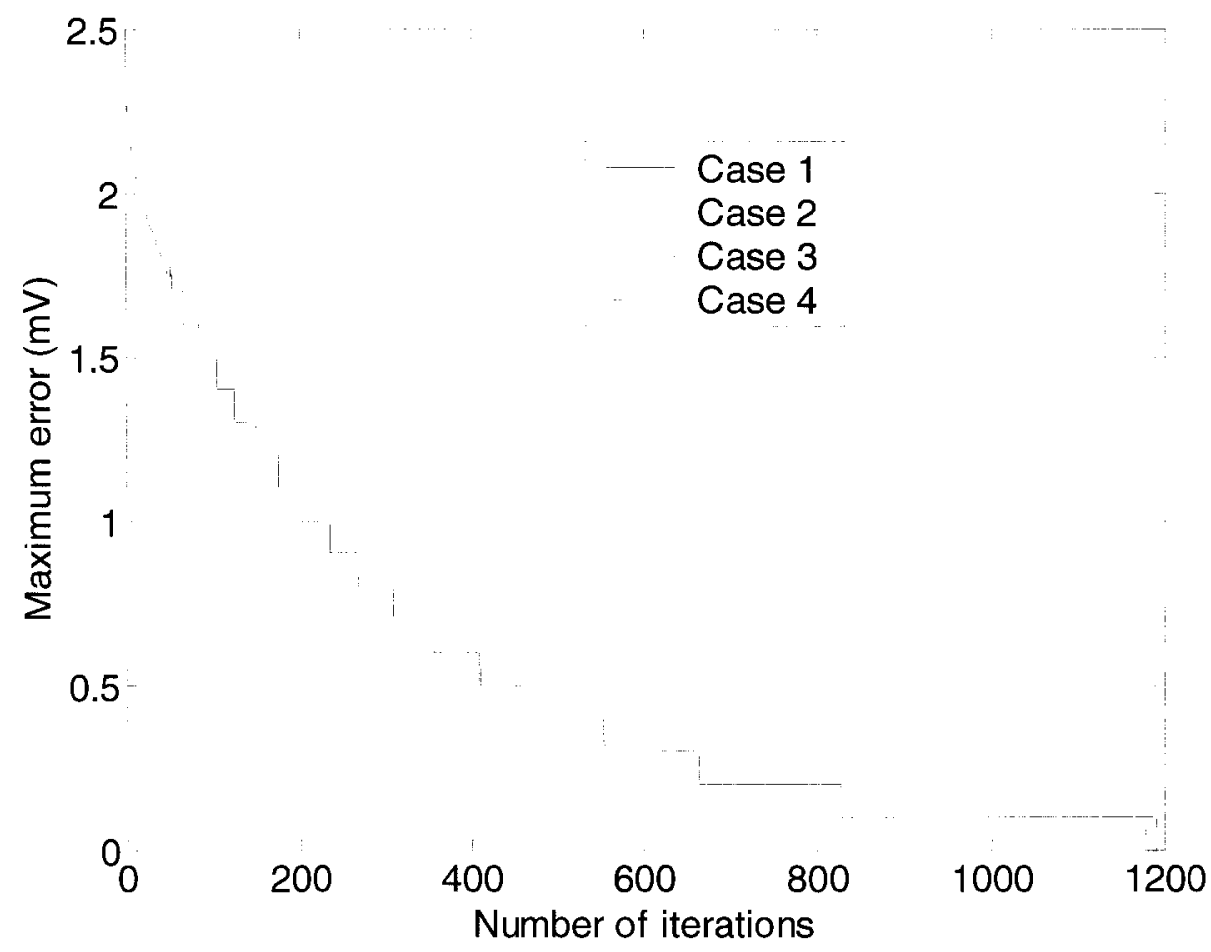

Fig. 5.22: Convergence process for different initial conditions 


\subsubsection{Limiting current density control factor}

To find a suitable limiting current density control factor, $\psi$, for cases with low limiting current density (e.g. $i_{L}=1 \times 10^{-8} \mathrm{~A} / \mathrm{mm}^{2}$ ), a numerical study is carried out. In Fig. 5.23, the part of the cathodic polarization curve (Eq. 3.18) that is close to the limiting current density, $i_{L}$, is plotted for the base case. In this figure, the horizontal axis shows $\left(\psi \times i_{L}\right)$ along which 1.0 corresponds to $i_{L}$, i.e. $\psi=1.0$. Since $\phi_{o a}$ must larger than $-780 \mathrm{mV}$, which is the anodic half cell potential that establishes the lower bound of the possible potentials on the steel surface, the polarization curve is plotted until $\psi=0.99995$.

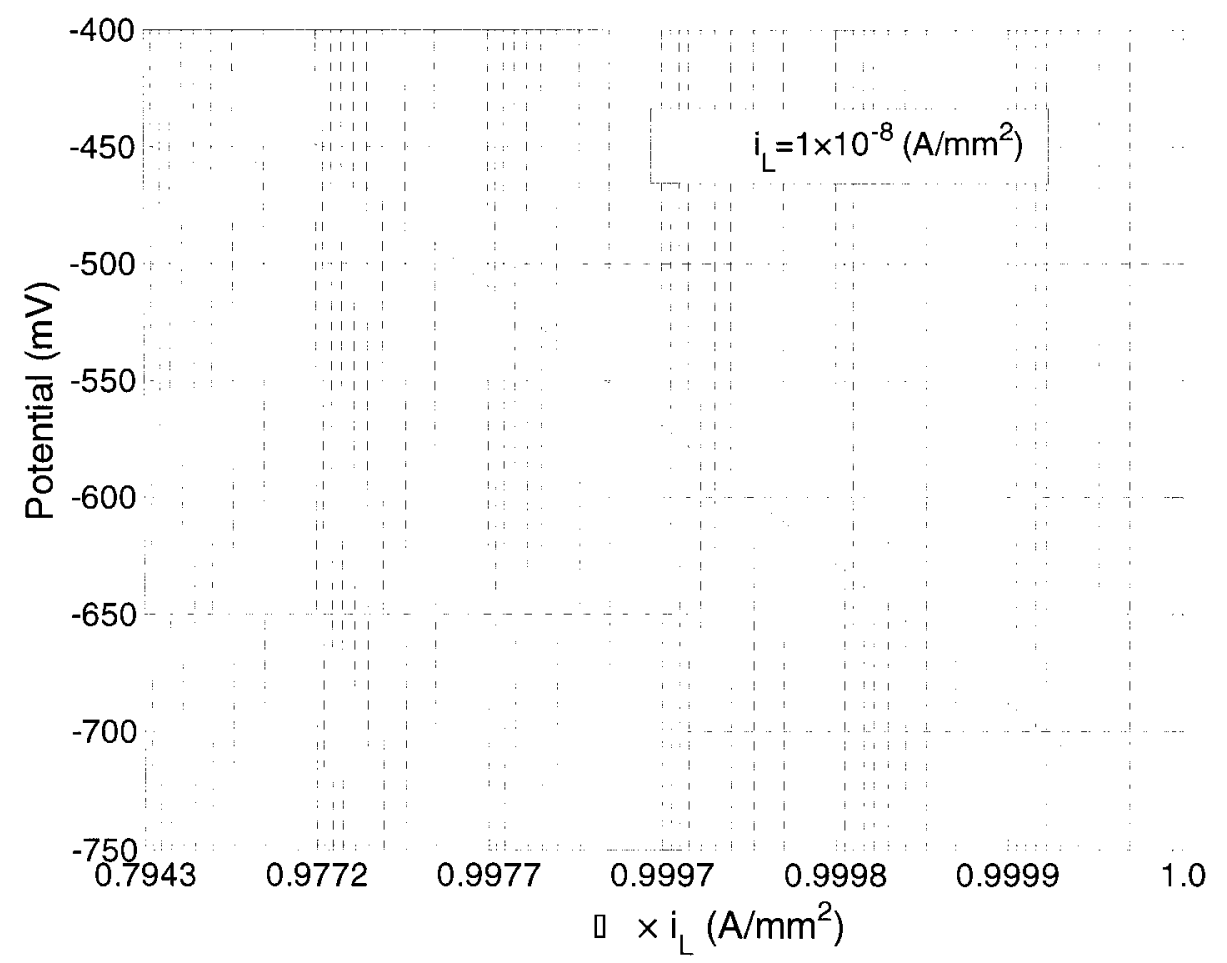

Fig. 5.23: The end part of the cathodic polarization curve

It is also known that the corrosion current density is close to $i_{L}$ when the limiting current density is low (Isgor and Razaqpur 2006); hence it also has a lower boundary; however the establishment of this lower boundary requires a numerical investigation. Table 5.6 
provides the analysis data and results for the limiting current density correction factor analysis. The results of this investigation are plotted in Figs. 5.24-5.26 for four cases which provided the smallest value of maximum error. As illustrated in these figures and presented in Table 5.6, there is no significant effect of the limiting current density correction factor on the results of the analysis; however, depending on the factor, the rate and success of convergence differ in each case. $\psi=0.995$ (Case \#5) provides the best results for convergence.

Table 5.6: Data and results of the limiting current density correction factor analysis

\begin{tabular}{|c|c|c|c|c|c|c|c|c|}
\hline Case \# & $\begin{array}{c}\text { Element } \\
\text { size } \\
(\mathbf{m m})\end{array}$ & $\begin{array}{c}\boldsymbol{r} \\
(\mathbf{o h m}-\mathbf{m m})\end{array}$ & $\mathbf{A} / \mathbf{C}$ & $\begin{array}{c}\boldsymbol{i}_{\boldsymbol{L}} \\
\left(\mathbf{A} / \mathbf{m m}^{2}\right)\end{array}$ & $\xi$ & $\psi$ & $\begin{array}{c}\text { Max. } \\
\text { Error }^{(\mathbf{1})} \\
(\mathbf{m V})\end{array}$ & $\begin{array}{c}\text { Corresponding } \\
\text { iteration }^{(2)}\end{array}$ \\
\hline 1 & 5 & 140000 & 0.111 & $10^{-8}$ & 0.003 & 0.99995 & $<0.3$ & 2296 \\
\hline 2 & 5 & 140000 & 0.111 & $10^{-8}$ & 0.003 & 0.9999 & $<0.3$ & 4419 \\
\hline 3 & 5 & 140000 & 0.111 & $10^{-8}$ & 0.003 & 0.999 & $<0.3$ & 1155 \\
\hline 4 & 5 & 140000 & 0.111 & $10^{-8}$ & 0.003 & 0.99 & $<0.1$ & 1192 \\
\hline 5 & 5 & 140000 & 0.111 & $10^{-8}$ & 0.003 & 0.995 & $<0.1$ & 1185 \\
\hline 6 & 5 & 140000 & 0.111 & $10^{-8}$ & 0.003 & 0.996 & $<0.1$ & 1257 \\
\hline 7 & 5 & 140000 & 0.111 & $10^{-8}$ & 0.003 & 0.997 & $<0.2$ & 1159 \\
\hline 8 & 5 & 140000 & 0.111 & $10^{-8}$ & 0.003 & 0.9965 & $<0.2$ & 1430 \\
\hline 9 & 5 & 140000 & 0.111 & $10^{-8}$ & 0.003 & 0.9964 & $<0.1$ & 1281 \\
\hline
\end{tabular}

(1) Smallest value of the maximum error after 5000 iterations

(2) The iteration corresponding to the smallest value of the maximum error 


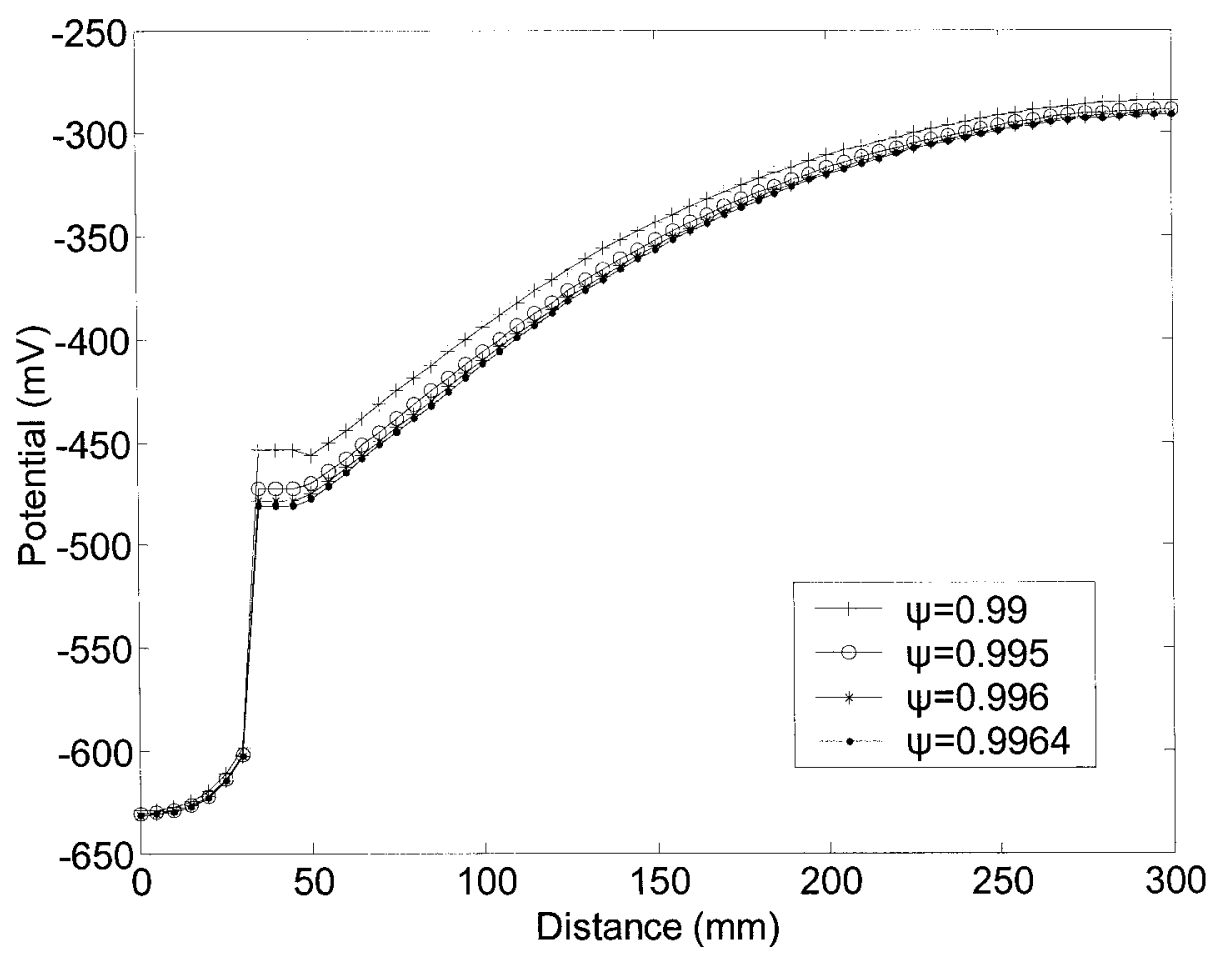

Fig. 5.24: Potential distribution on the rebar for various values of $\psi$

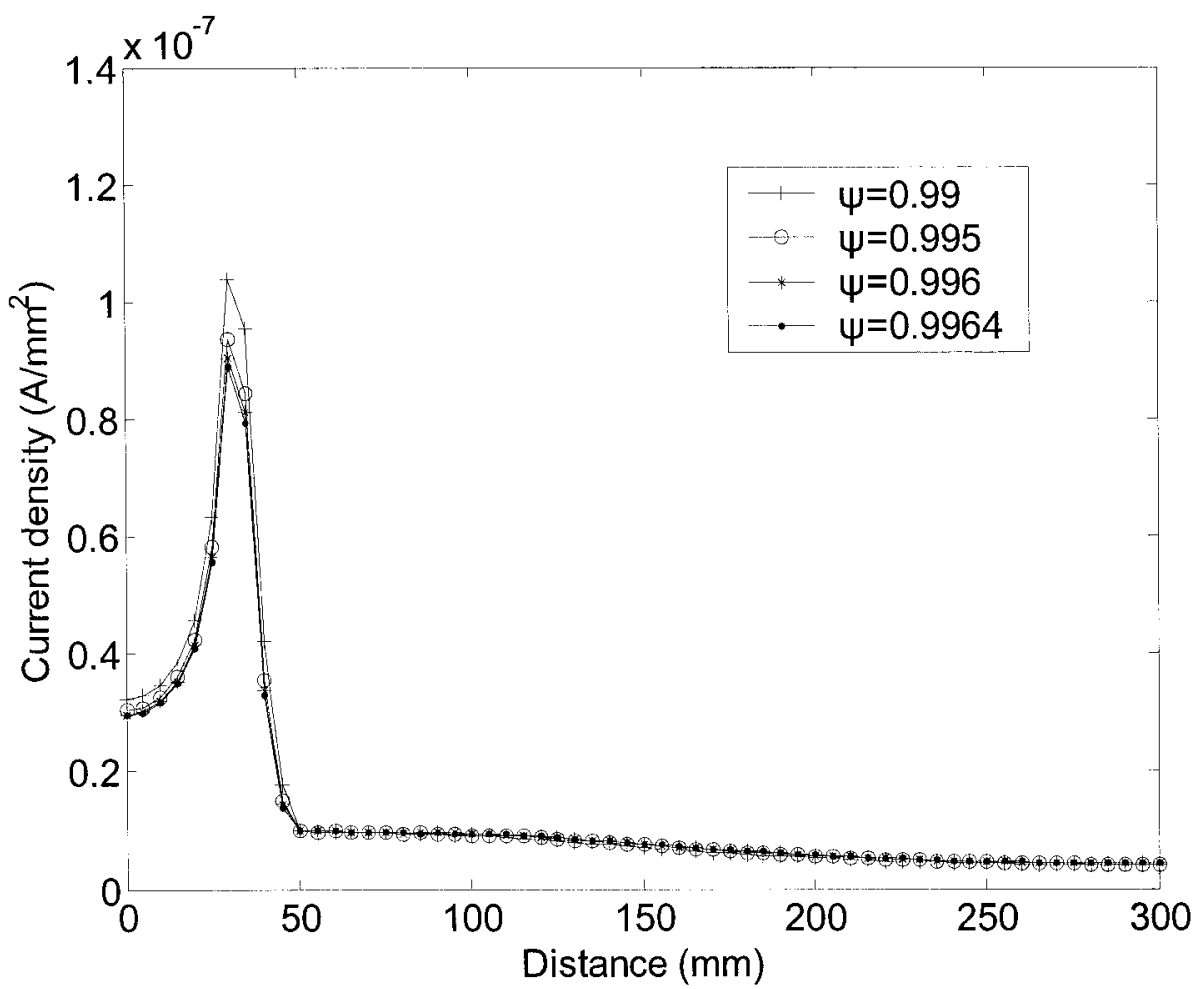

Fig. 5.25: Current density distribution on the rebar for various values of $\psi$ 


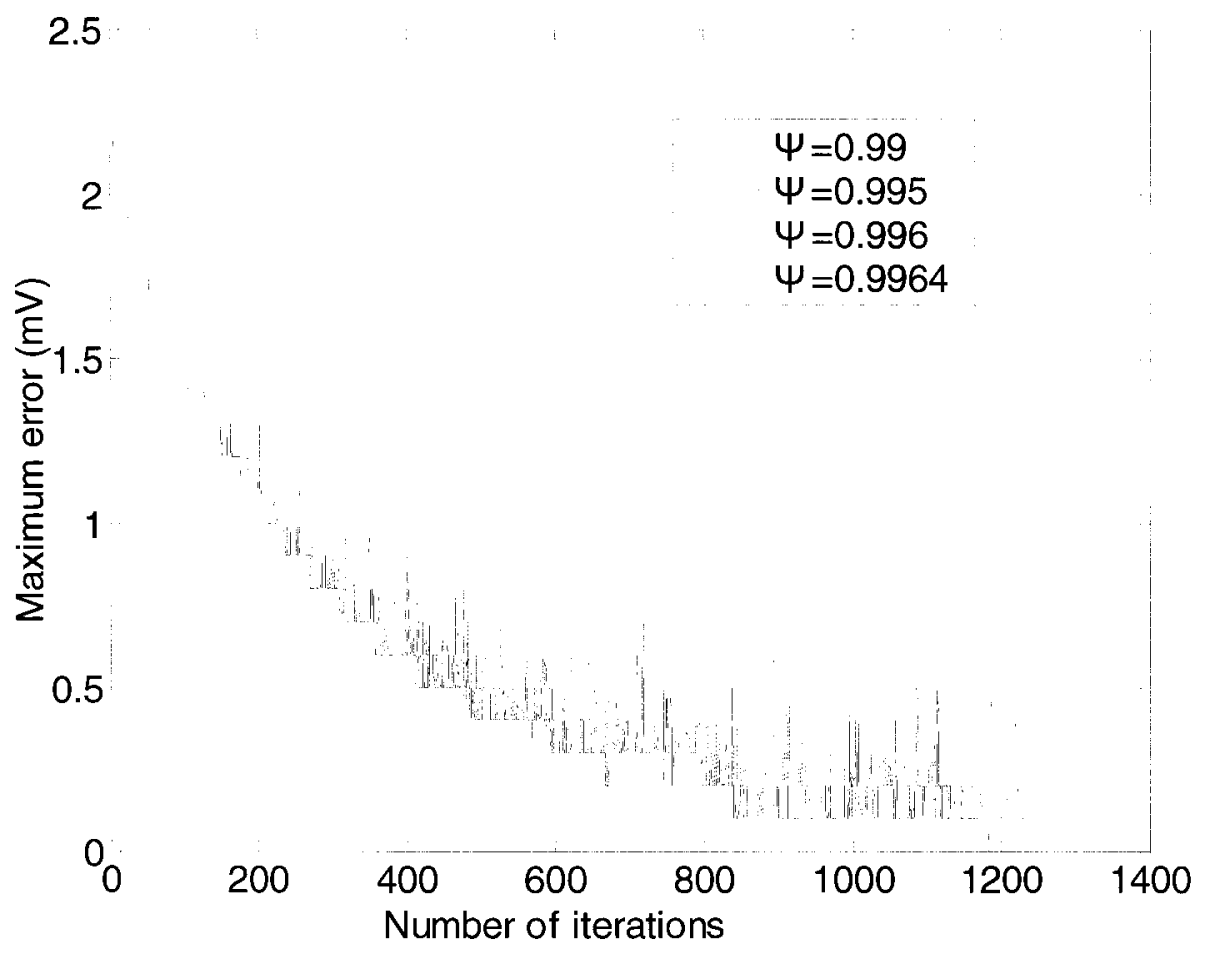

Fig. 5.26: Convergence process for various values of $\psi$

It should be noted that the plateau in the potential curve in Fig. 5.24 is a numerical error which originates from using one limiting density correction factor for all nodes along the steel surface. Although this plateau does not constitute a concern in most modelling studies, possible ways of its elimination is also studied in this research. It is found that using two values for the limiting current density control factor instead of one helps to obtain a smoother curve. The use of two factors is demonstrated in the following:

(1) The current densities corresponding to two factors are calculated as:

$i_{1}=\Psi_{1} i_{L}$

$i_{2}=\Psi_{2} i_{L}$

where $i_{1}$ and $i_{2}$ are the current densities calculated by control factors $\Psi_{1}$ and $\Psi_{2}$, 
respectively.

(2) Every calculated current density that is larger than $i_{L}$ is corrected by $i_{1}$ and $i_{2}$ using:

$i_{\text {correction }}=i_{1}+\lambda\left(i_{\text {calculated }}-i_{2}\right)$

where $i_{\text {correction }}$ is the corrected current density, $i_{\text {calculated }}$ is the calculated current density, which is larger than $i_{L}$, and $\lambda$ is the transition ratio which is calculated from:

$\lambda=\left(i_{2}-i_{1}\right) /\left(i_{\max , \text { calcultated }}-i_{1}\right)$

where $i_{\text {max,calculated }}$ is the largest calculated current density among all nodes along the cathodic region. Note that $i_{2}$ must always be larger than $i_{1}$ to keep the corrected current density smaller than the limiting current density.

Figure 5.27 illustrates the potential distribution curves presented in Fig.5.24 after the two factors are used. For all cases presented in this figure, the maximum errors are found to be less than $0.1 \mathrm{mV}$ which are achieved at around 1200 iterations. 


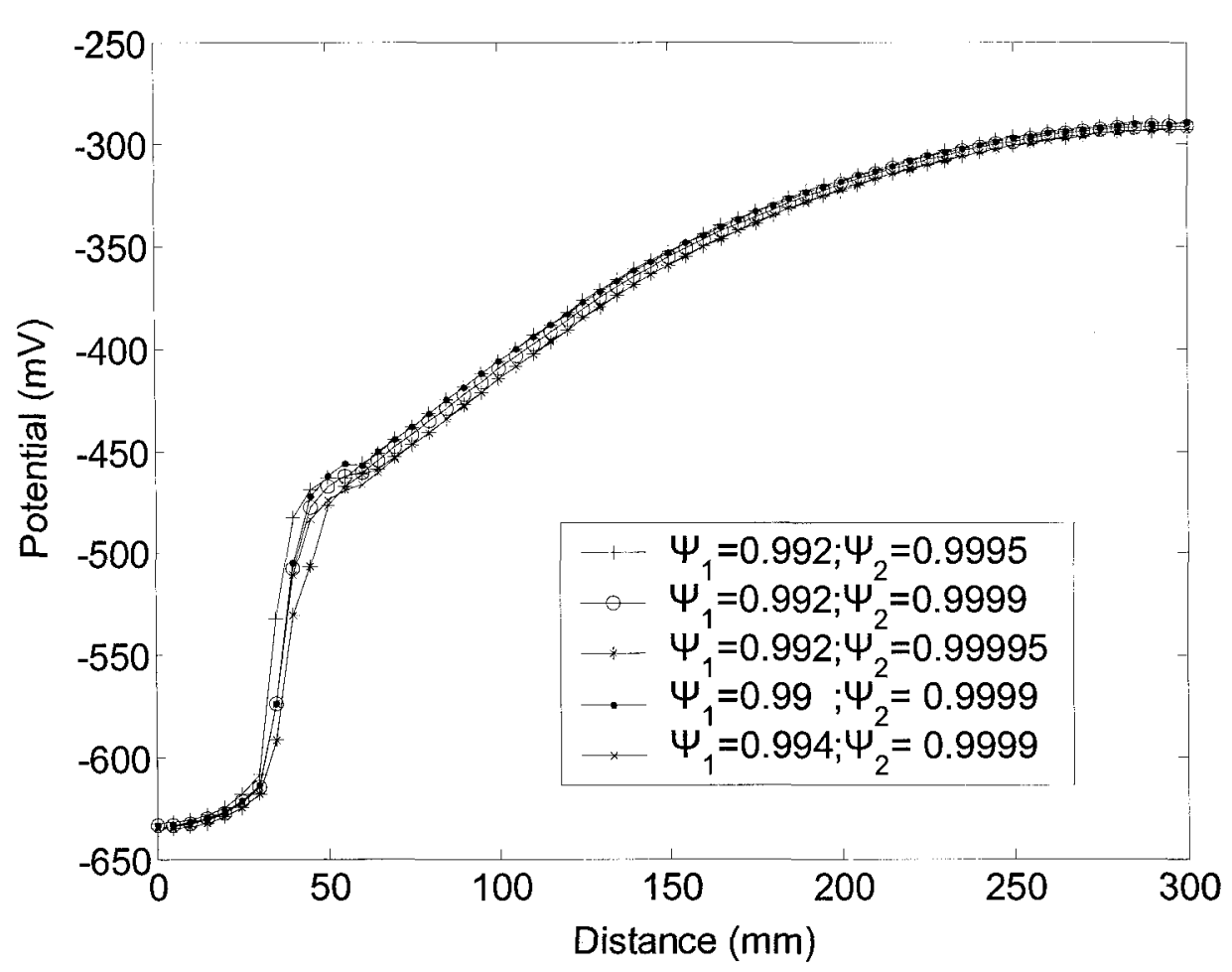

Fig. 5.27: Potential distribution on the rebar when two $\psi$ values are used

The effect of limiting current density on the solution using the MDIM is also studied. Table 5.7 shows the input data and results of this investigation. Figures 5.28 and 5.39 illustrate the potential distribution and current density on the steel surface for various values of $i_{L}$. It can be observed from Table 5.7 and Figs. 5.28 and 5.29 that, when the limiting current density is large (i.e. in the order of $10^{-7}$ and higher), there is no need for using a $\psi$ factor. In addition, only one $\psi$ factor is found to be adequate to obtain a smooth potential distribution on the steel surface for cases with limiting current density of $5 \times 10^{-8} \mathrm{~A} / \mathrm{mm}^{2}$ and higher. For lower values of $i_{L}$, two factors are needed for a smooth curve. In Fig. 5.30, it is illustrated that, even after the limiting current density correction factors are used, the convergence of cases with low $i_{L}$ values show fluctuations during successive iterations; however, the use of the factors prevent any possible divergence. 
Unlike the DIM, the MDIM results in a smooth potential distribution in the domain of the analysis as shown in Figs. 31-33.

Table 5.7: The effect of limiting current density on the solution using MDIM

\begin{tabular}{|c|c|c|c|c|c|c|c|c|c|}
\hline Case & $\begin{array}{c}\text { Element } \\
\text { size } \\
(\mathbf{m m})\end{array}$ & $\begin{array}{c}\boldsymbol{r} \\
(\mathbf{o h m}-\mathbf{m m})\end{array}$ & $\mathbf{A} / \mathbf{C}$ & $\begin{array}{c}i_{L} \\
\left(\mathbf{A} / \mathbf{m m}^{2}\right)\end{array}$ & $\xi$ & $\psi_{1}{ }^{(1)}$ & $\psi_{2}{ }^{(\mathbf{1})}$ & $\begin{array}{c}\text { Max. } \\
\text { Error }^{(2)} \\
(\mathbf{m V})\end{array}$ & $\begin{array}{c}\text { Corresponding } \\
\text { iteration }^{(3)}\end{array}$ \\
\hline 1 & 5 & 140000 & 0.111 & $10^{-6}$ & 0.003 & N/A & N/A & $<0.1$ & 1189 \\
\hline 2 & 5 & 140000 & 0.111 & $10^{-7}$ & 0.003 & N/A & N/A & $<0.1$ & 1200 \\
\hline 3 & 5 & 140000 & 0.111 & $9 \times 10^{-8}$ & 0.003 & N/A & N/A & $<0.1$ & 1199 \\
\hline 4 & 5 & 140000 & 0.111 & $7 \times 10^{-8}$ & 0.003 & 0.9 & 0.9 & $<0.1$ & 1170 \\
\hline 5 & 5 & 140000 & 0.111 & $5 \times 10^{-8}$ & 0.003 & 0.9 & 0.9 & $<0.1$ & 1173 \\
\hline 6 & 5 & 140000 & 0.111 & $3 \times 10^{-8}$ & 0.003 & 0.9 & 0.99 & $<0.1$ & 1181 \\
\hline 7 & 5 & 140000 & 0.111 & $1 \times 10^{-8}$ & 0.003 & 0.992 & 0.9999 & $<0.1$ & 1201 \\
\hline
\end{tabular}

(1) Two factors are used when there is a plateau in potential distribution on steel surface

(2) Smallest value of the maximum error after 5000 iterations

(3) The iteration corresponding to the smallest value of the maximum error

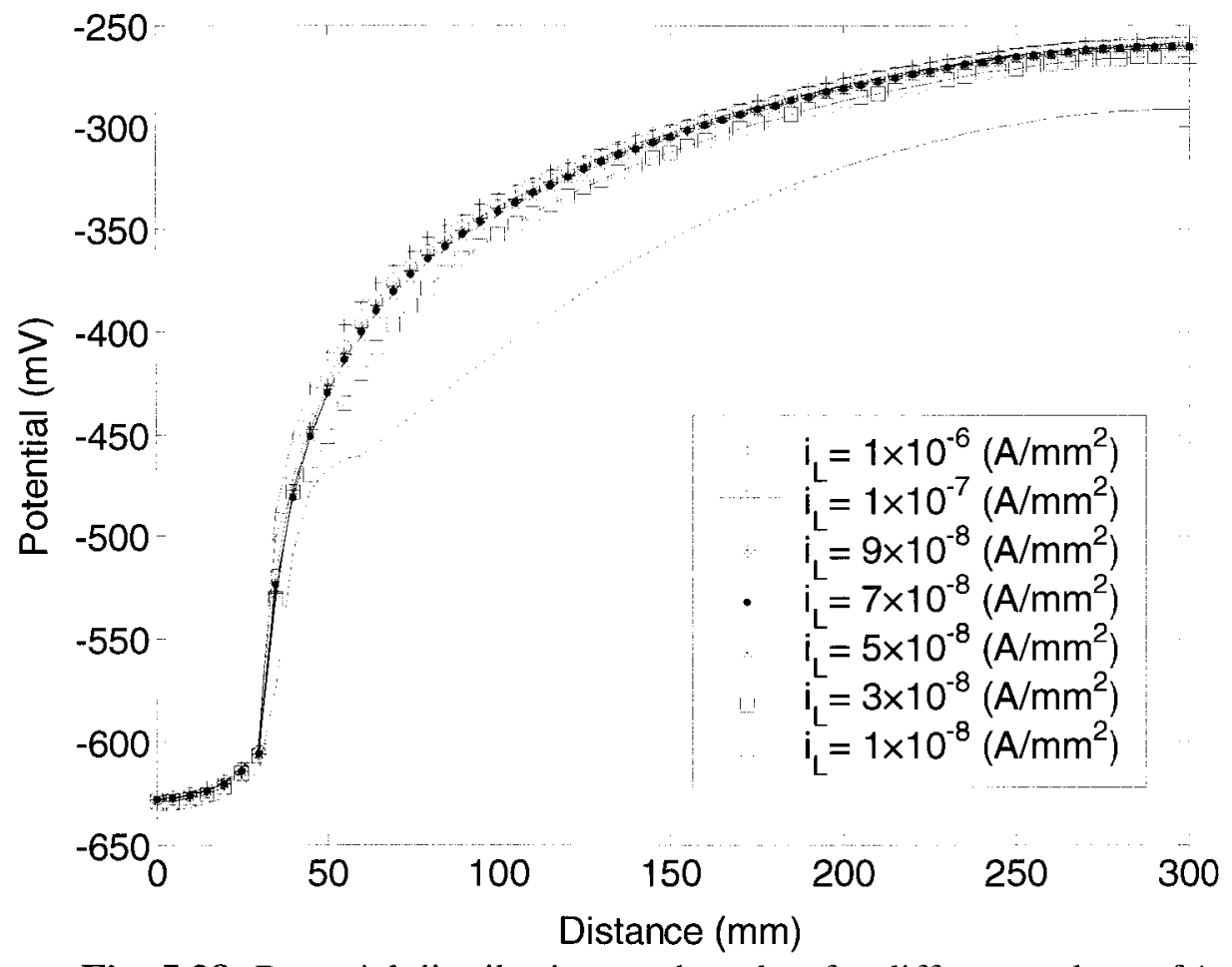

Fig. 5.28: Potential distribution on the rebar for different values of $i_{L}$ 


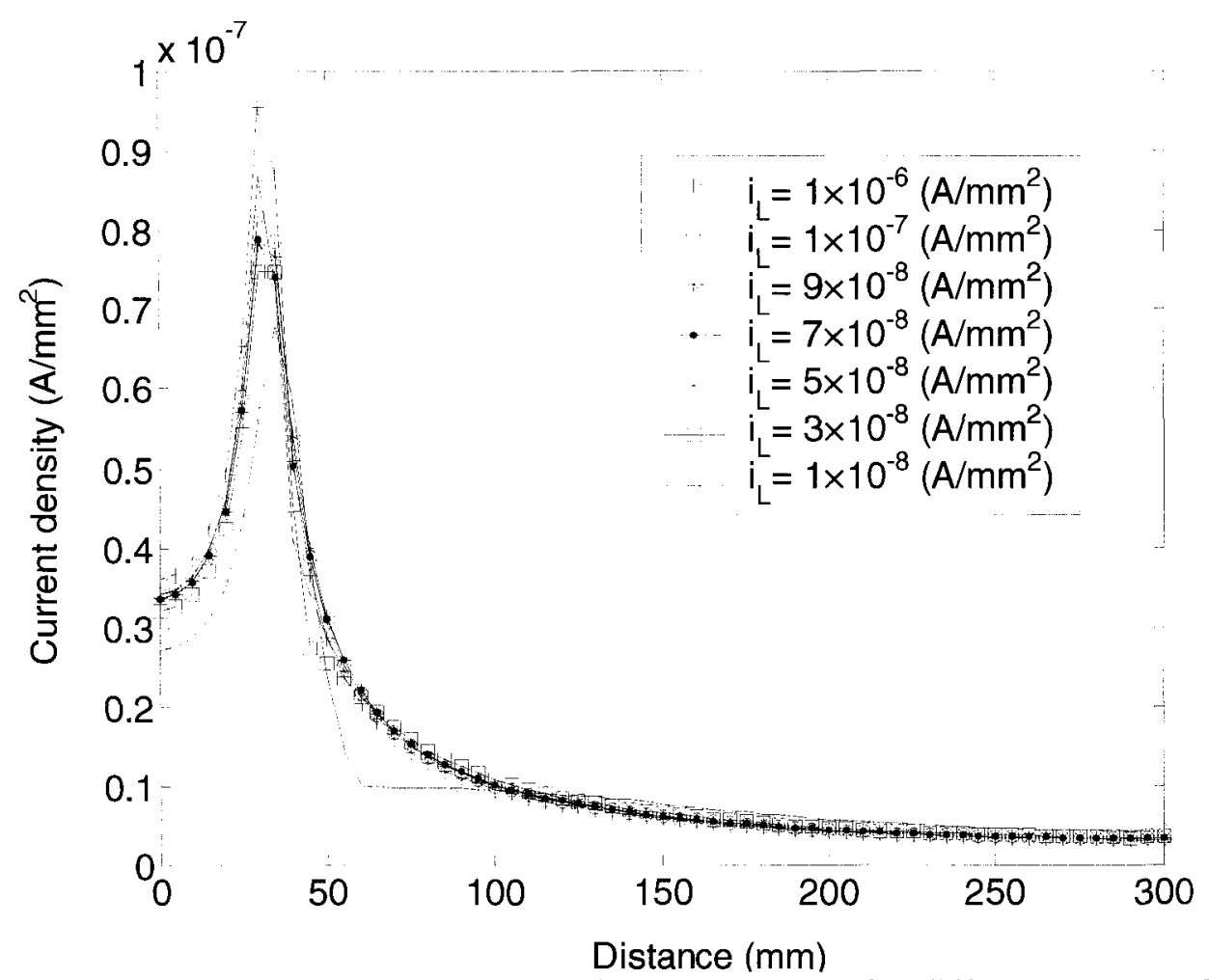

Fig. 5.29: Current density distribution on the rebar for different values of $i_{L}$

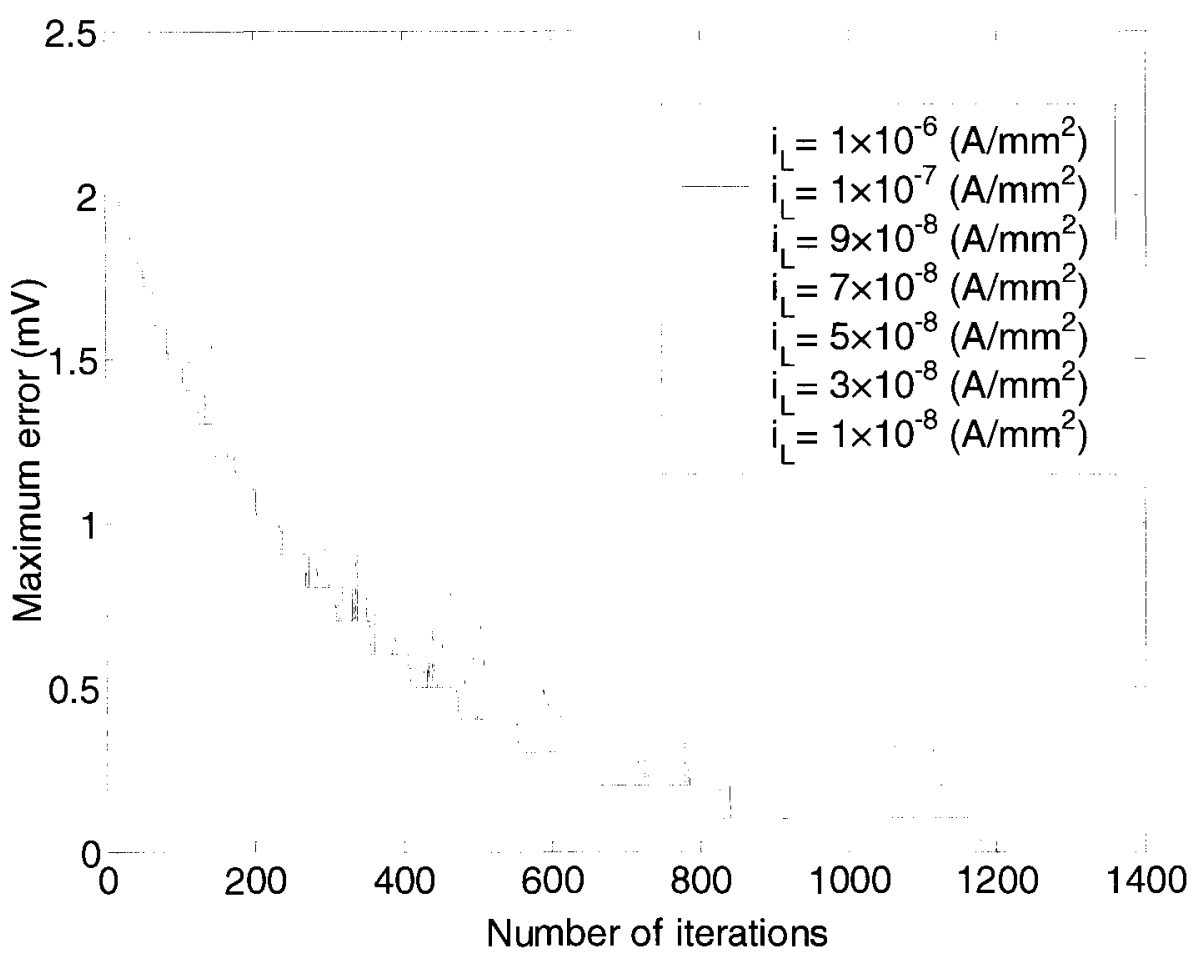

Fig. 5.30: Convergence process for different values of $i_{L}$ 

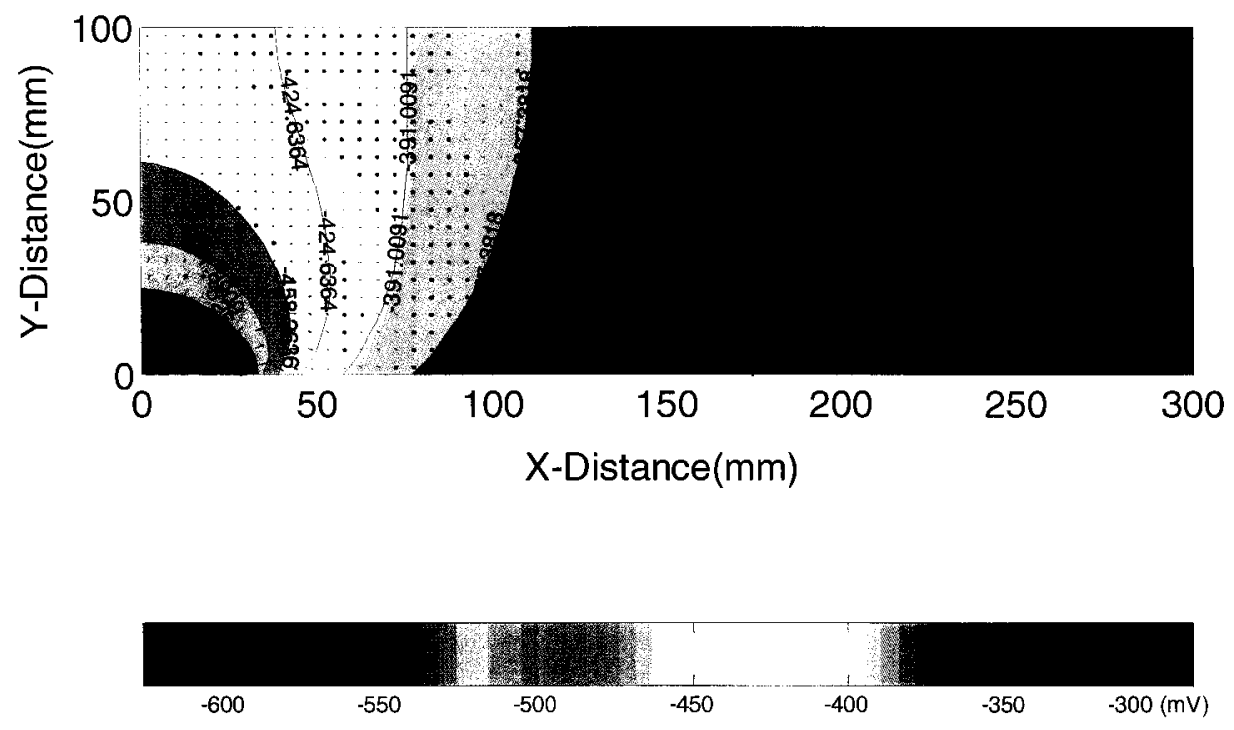

Fig. 5.31:Potential and current density distribution in the domain calculated by using the MDIM $\left(i_{L}=1 \times 10^{-6} \mathrm{~A} / \mathrm{mm}^{2}\right)$
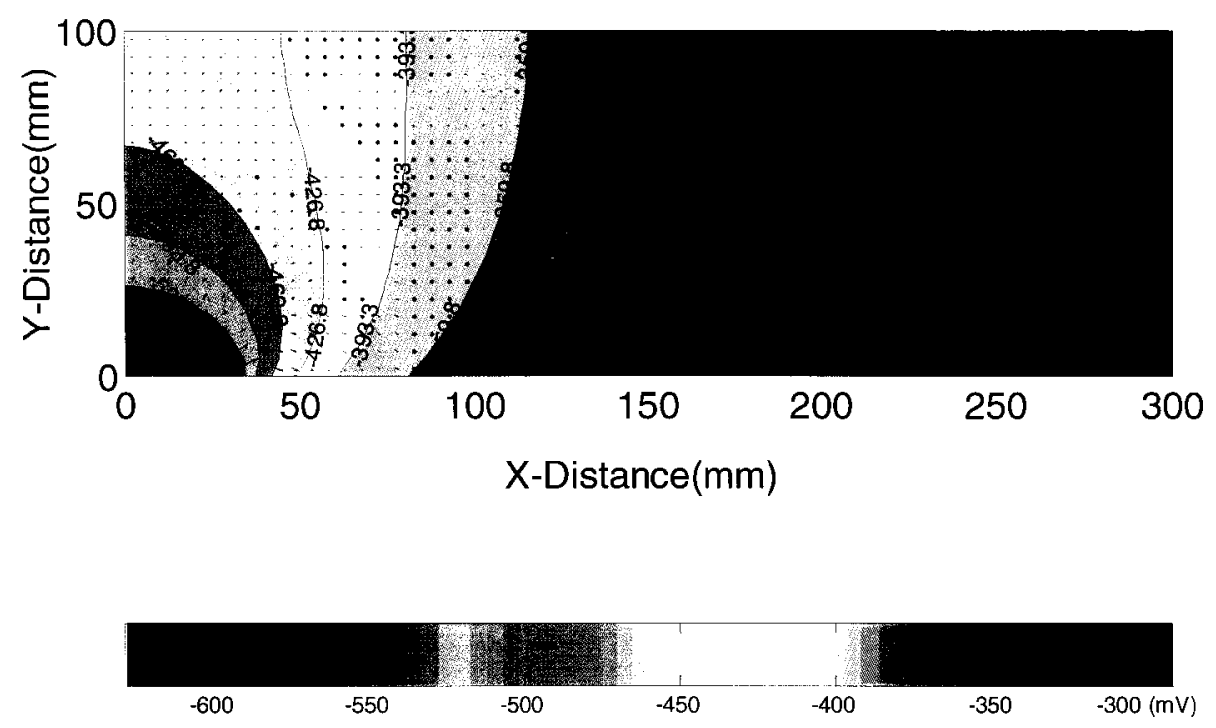

Fig. 5.32:Potential and current density distribution in the domain calculated by using the MDIM $\left(i_{L}=1 \times 10^{-7} \mathrm{~A} / \mathrm{mm}^{2}\right)$ 

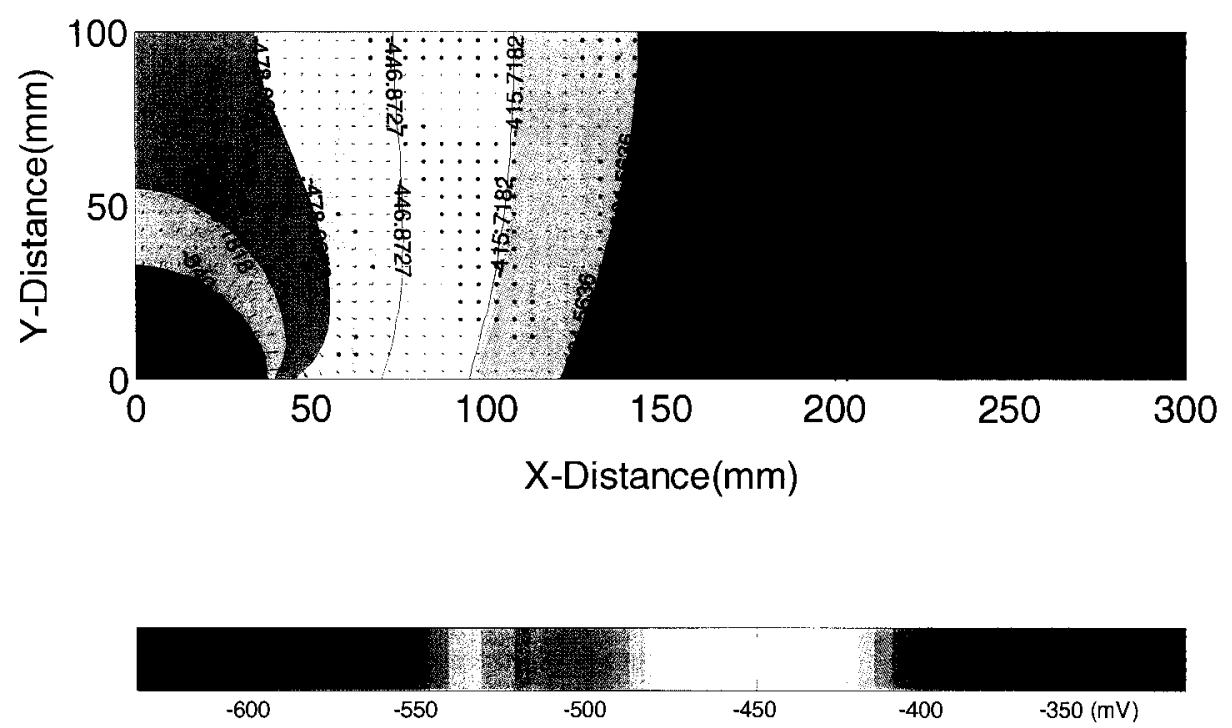

Fig. 5.33: Potential and current density distribution in the domain calculated by using the $\operatorname{MDIM}\left(i_{L}=1 \times 10^{-8} \mathrm{~A} / \mathrm{mm}^{2}\right)$

\subsubsection{Element size analysis}

To investigate the effect of element size on the solution of steel corrosion in concrete, numerical simulations are carried on the base case with varying element sizes under three different limiting current density values (i.e. $i_{L}=1 \times 10^{-6}, 1 \times 10^{-7}$ and $1 \times 10^{-8} \mathrm{~A} / \mathrm{mm}^{2}$ ). The input data and the results of the analysis are presented in Table 5.8. Figures 5.34-5.36, 5.37-5.39, 5.40-5.42, respectively illustrate the potential distribution, current density and convergence process for different limiting current densities.

The convergence of all cases except for the cases with 2 mm-elements show smooth convergence, as shown in Figs. 5.40-5.42. It is a common problem in corrosion analysis to observe divergence as the element size becomes smaller. This phenomenon, commonly referred to as the size effect, is due to the increased number of nodes for which the 
convergence is tested. The convergence also becomes harder to achieve as the limiting current density becomes smaller. Although the limiting current density factor prevents divergence for larger elements in cases with low limiting current density, it fails to control convergence for 2-mm elements. An important observation can be made from Figs. 5.40-5.42 and Table 5.8 that, for converged cases, the element size does not have a significant effect on convergence rate.

For converged cases, the potential distributions in Figs. 5.34-5.36 obtained for element sizes are nearly the same. The fluctuations observed for 2 -mm elements demonstrate the failed convergence process. On the other hand, the current density can be different, especially the anode-cathode interface, for varying element sizes, as illustrated in Figs. 5.37-5.39. Once again, the fluctuations in the current densities in cases with 2-mm elements show the failed convergence process. The accuracy of the solution is better at the anode-to-cathode interface as the element size becomes smaller; however, the smaller element size implies the convergence is harder to achieve. Therefore in selecting the element size, a fine balance between the accuracy and convergence should be found. Based on the results of this investigation, using 5-mm elements is found to be appropriate; hence this element size is used for the remainder of this study. 
Table 5.8: Element size analysis data and results by the MDIM

\begin{tabular}{|c|c|c|c|c|c|c|c|c|c|}
\hline Case & $\begin{array}{c}\text { Element } \\
\text { size } \\
(\mathbf{m m})\end{array}$ & $\begin{array}{c}\boldsymbol{r} \\
(\mathbf{o h m}-\mathbf{m m})\end{array}$ & $\mathbf{A} / \mathbf{C}$ & $\begin{array}{c}\boldsymbol{i}_{L} \\
\left(\mathbf{A} / \mathbf{m m}^{2}\right)\end{array}$ & $\xi$ & $\psi_{\mathbf{1}}^{(\mathbf{1})}$ & $\psi_{\mathbf{2}}^{(\mathbf{1})}$ & $\begin{array}{c}\text { Max. }_{\text {Error }}^{(\mathbf{2})} \\
(\mathbf{m V})\end{array}$ & $\begin{array}{c}\text { Corresponding } \\
\text { iteration }^{(3)}\end{array}$ \\
\hline 1 & 2 & 140000 & 0.111 & $10^{-6}$ & 0.003 & N/A & N/A & $<0.1$ & 1878 \\
\hline 2 & 5 & 140000 & 0.111 & $10^{-6}$ & 0.003 & N/A & N/A & $<0.1$ & 1171 \\
\hline 3 & 10 & 140000 & 0.111 & $10^{-6}$ & 0.003 & N/A & N/A & $<0.1$ & 1170 \\
\hline 4 & 15 & 140000 & 0.111 & $10^{-6}$ & 0.003 & N/A & N/A & $<0.1$ & 1161 \\
\hline 5 & 2 & 140000 & 0.111 & $10^{-7}$ & 0.003 & N/A & N/A & 0.2 & 1175 \\
\hline 6 & 5 & 140000 & 0.111 & $10^{-7}$ & 0.003 & N/A & N/A & $<0.1$ & 1201 \\
\hline 7 & 10 & 140000 & 0.111 & $10^{-7}$ & 0.003 & N/A & N/A & $<0.1$ & 1173 \\
\hline 8 & 15 & 140000 & 0.111 & $10^{-7}$ & 0.003 & N/A & N/A & $<0.1$ & 1161 \\
\hline 9 & 2 & 140000 & 0.111 & $10^{-8}$ & 0.003 & 0.9999 & 0.992 & 0.1 & 1160 \\
\hline 10 & 5 & 140000 & 0.111 & $10^{-8}$ & 0.003 & 0.9999 & 0.992 & $<0.1$ & 1174 \\
\hline 11 & 10 & 140000 & 0.111 & $10^{-8}$ & 0.003 & 0.9999 & 0.992 & $<0.1$ & 1192 \\
\hline 12 & 15 & 140000 & 0.111 & $10^{-8}$ & 0.003 & 0.9999 & 0.992 & $<0.1$ & 1030 \\
\hline
\end{tabular}

(1) Two factors are used when there is a plateau in potential distribution on steel surface

(2) Smallest value of the maximum error after 5000 iterations

(3) The iteration corresponding to the smallest value of the maximum error 


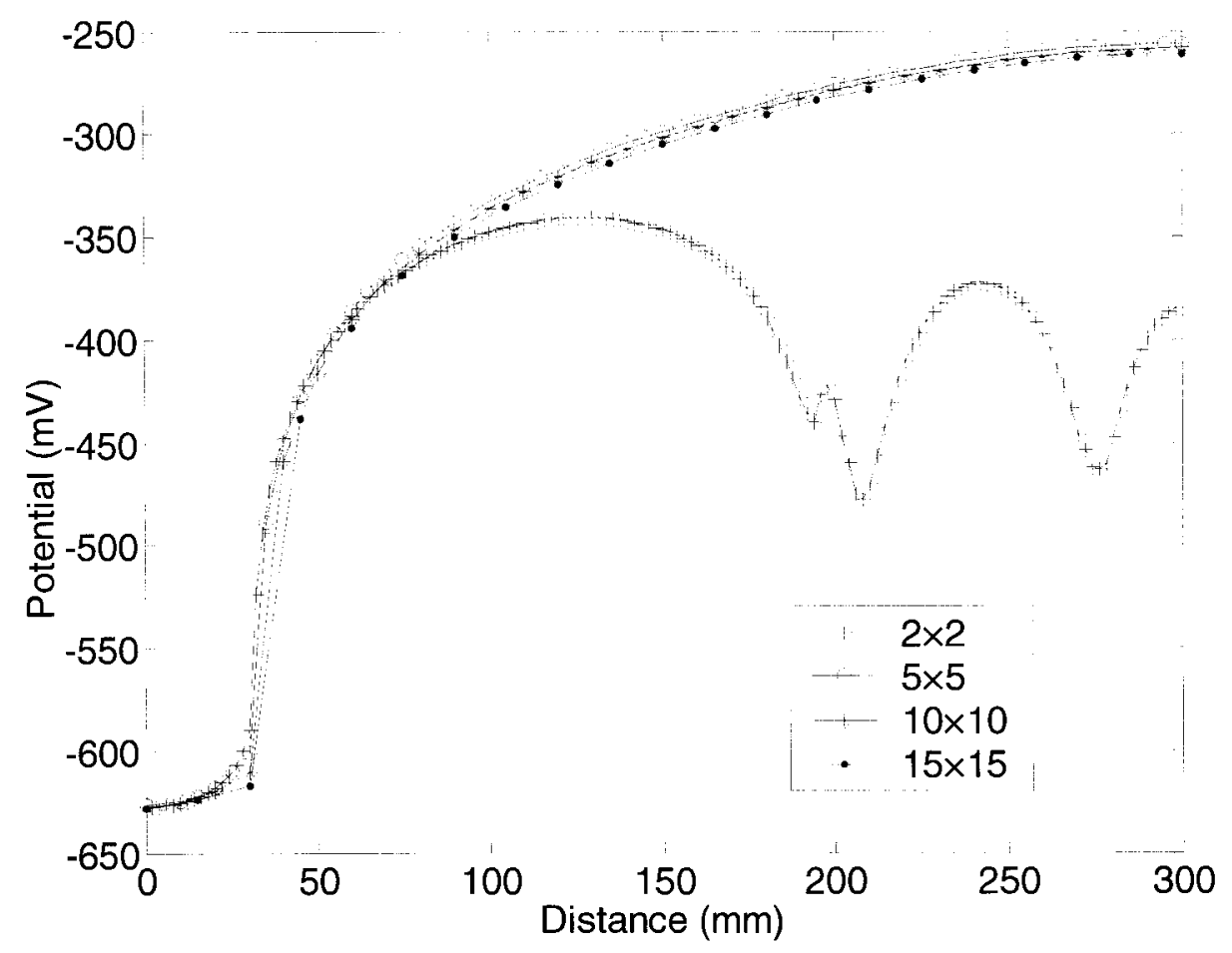

Fig. 5.34: Potential distribution on the rebar for $i_{L}=1 \times 10^{-6} \mathrm{~A} / \mathrm{mm}^{2}$ (Element size analysis)

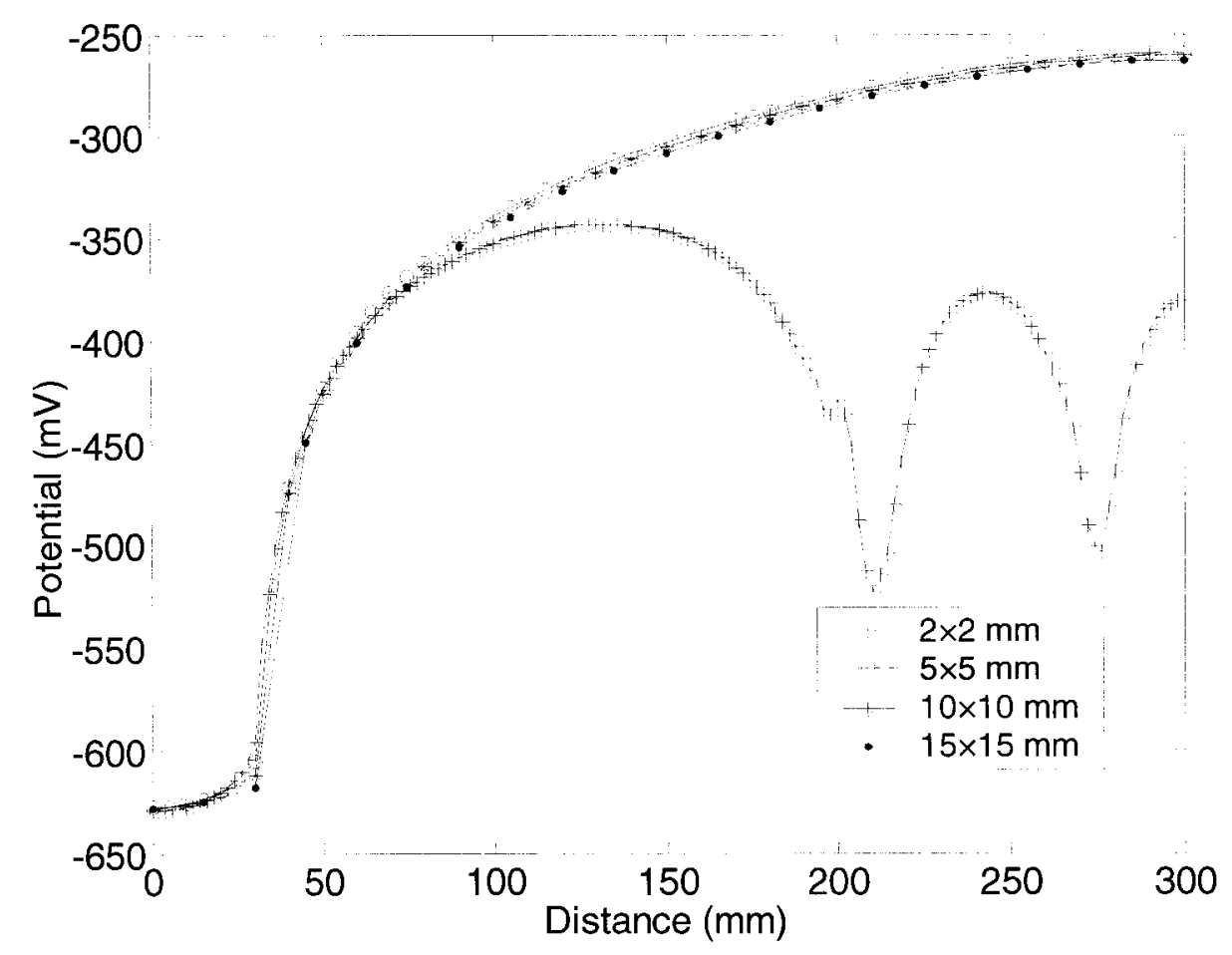

Fig. 5.35: Potential distribution on the rebar for $i_{L}=1 \times 10^{-7} \mathrm{~A} / \mathrm{mm}^{2}$ (Element size analysis) 


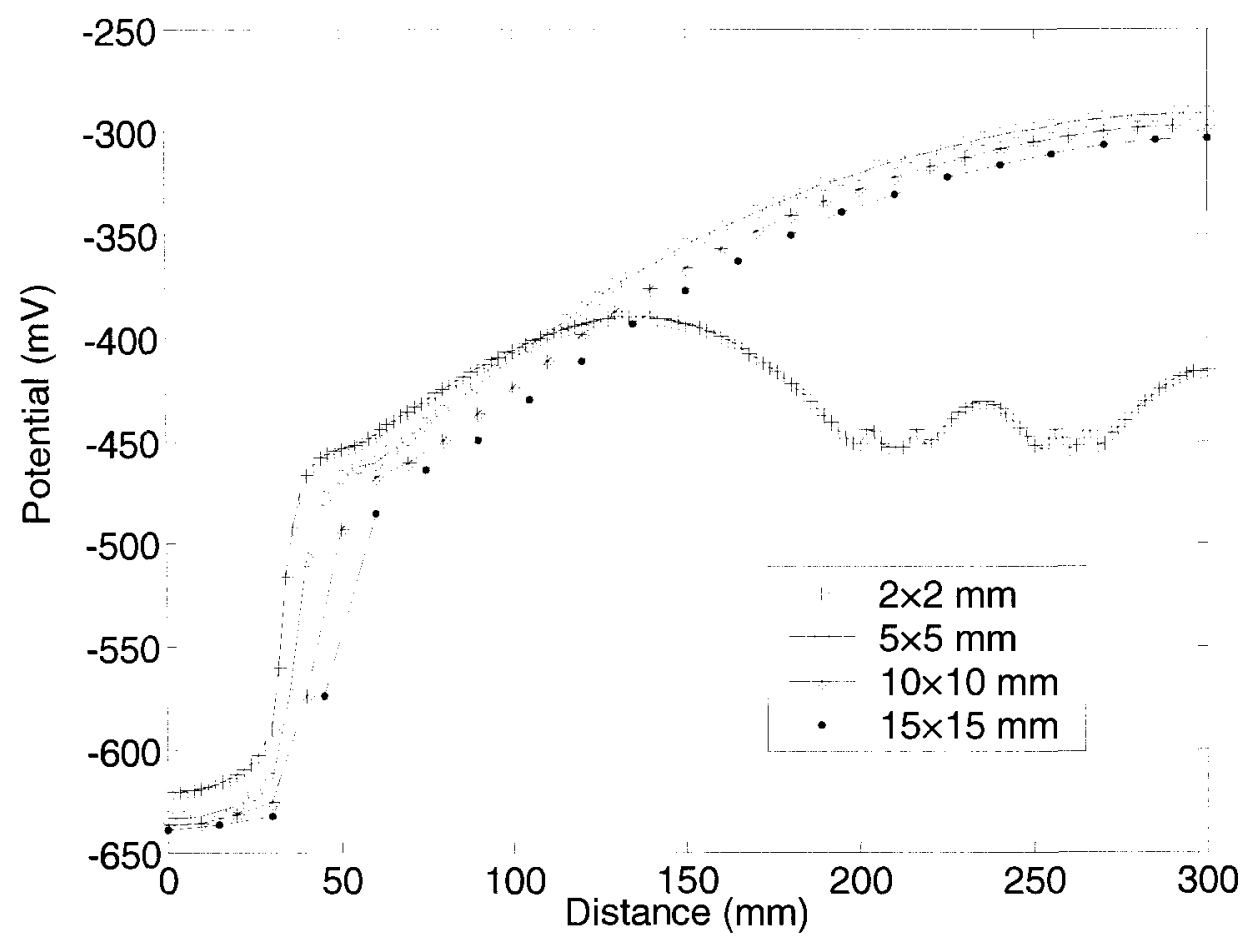

Fig. 5.36: Potential distribution on the rebar for $i_{L}=1 \times 10^{-8} \mathrm{~A} / \mathrm{mm}^{2}$

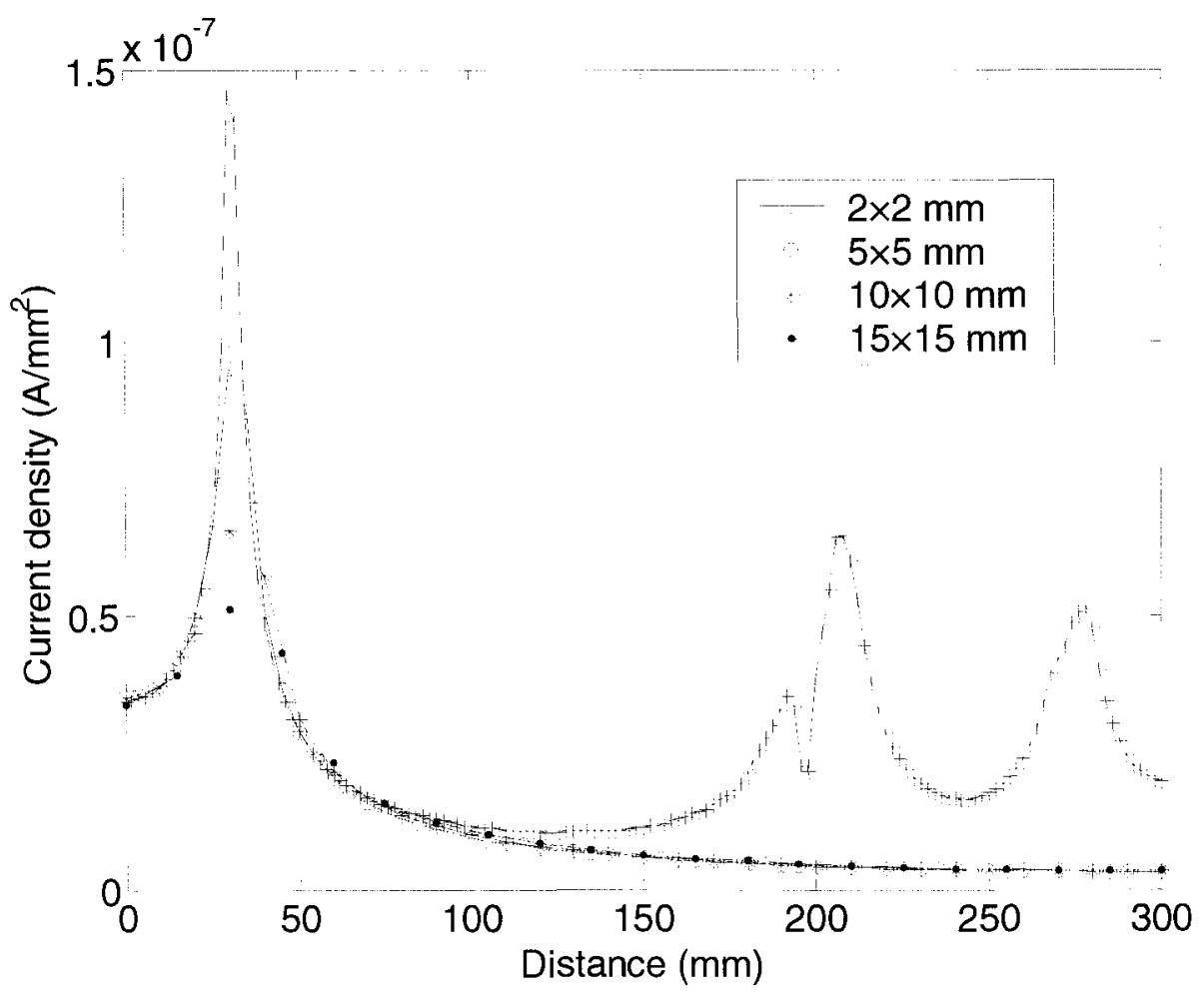

Fig. 5.37: Current density distribution on the rebar for $i_{L}=1 \times 10^{-6} \mathrm{~A} / \mathrm{mm}^{2}$ (Element size analysis) 


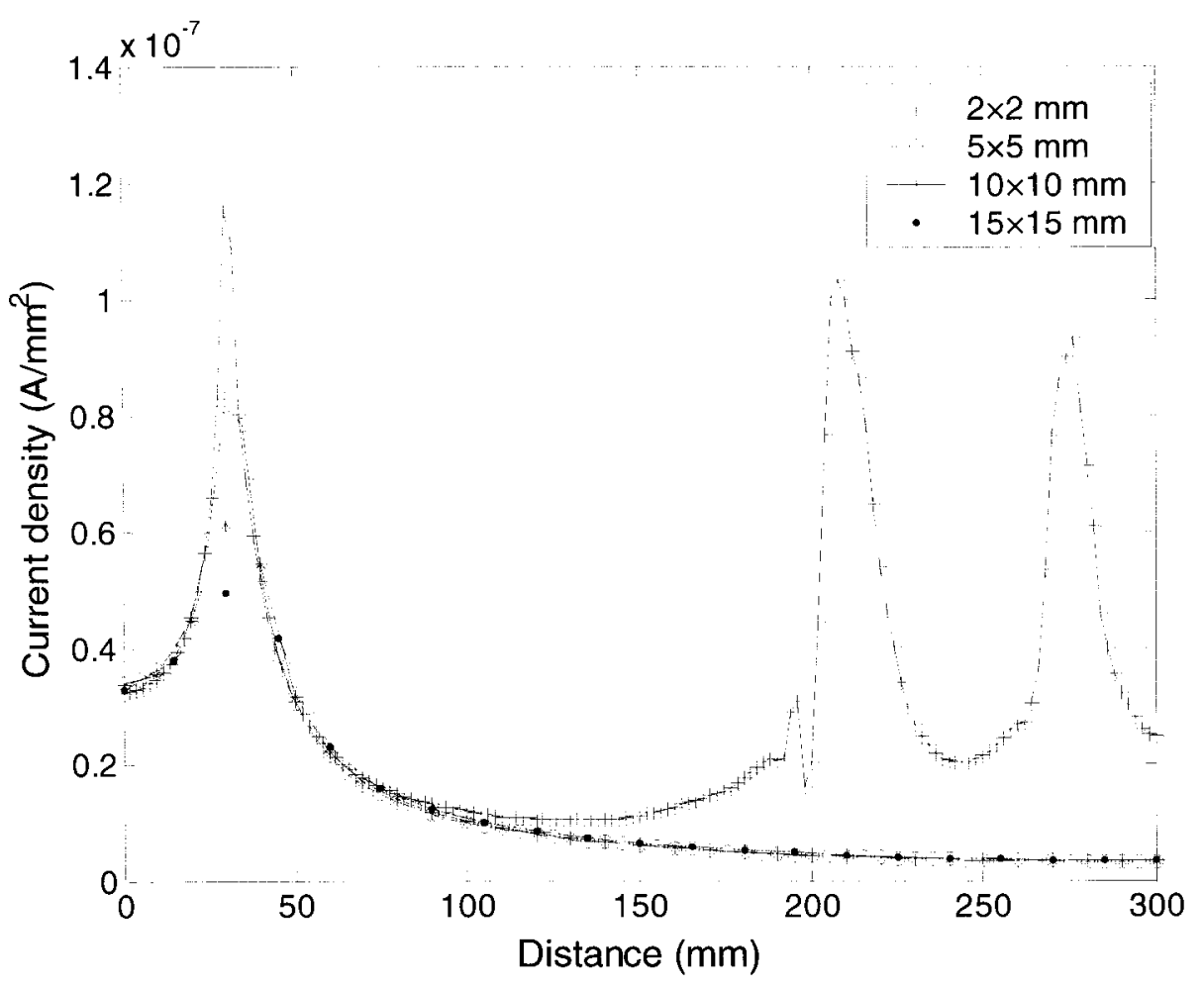

Fig. 5.38: Current density distribution on the rebar for $i_{L}=1 \times 10^{-7} \mathrm{~A} / \mathrm{mm}^{2}$ (Element size analysis)

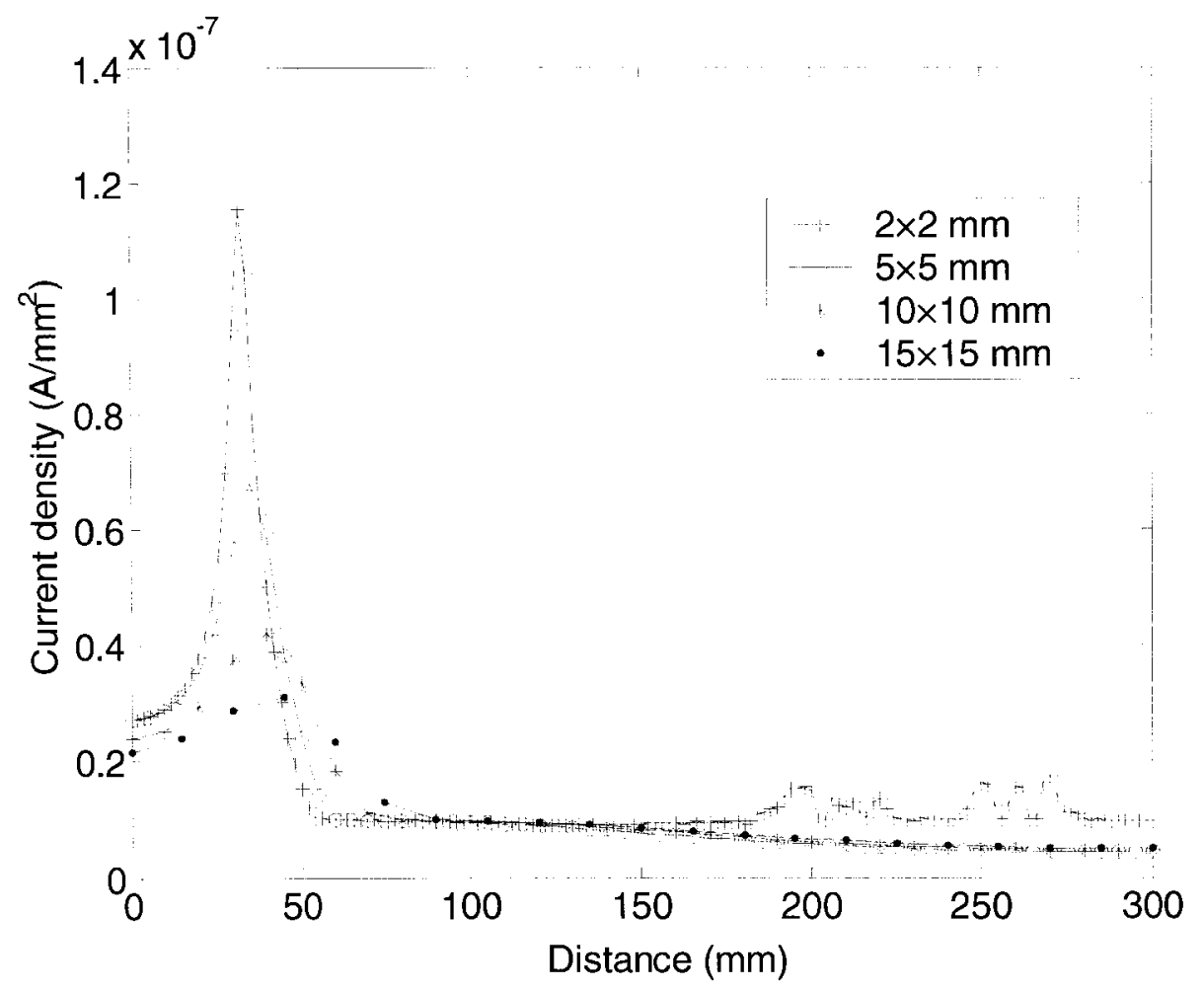

Fig. 5.39: Current density distribution on the rebar for $i_{L}=1 \times 10^{-8} \mathrm{~A} / \mathrm{mm}^{2}$ (Element size analysis) 


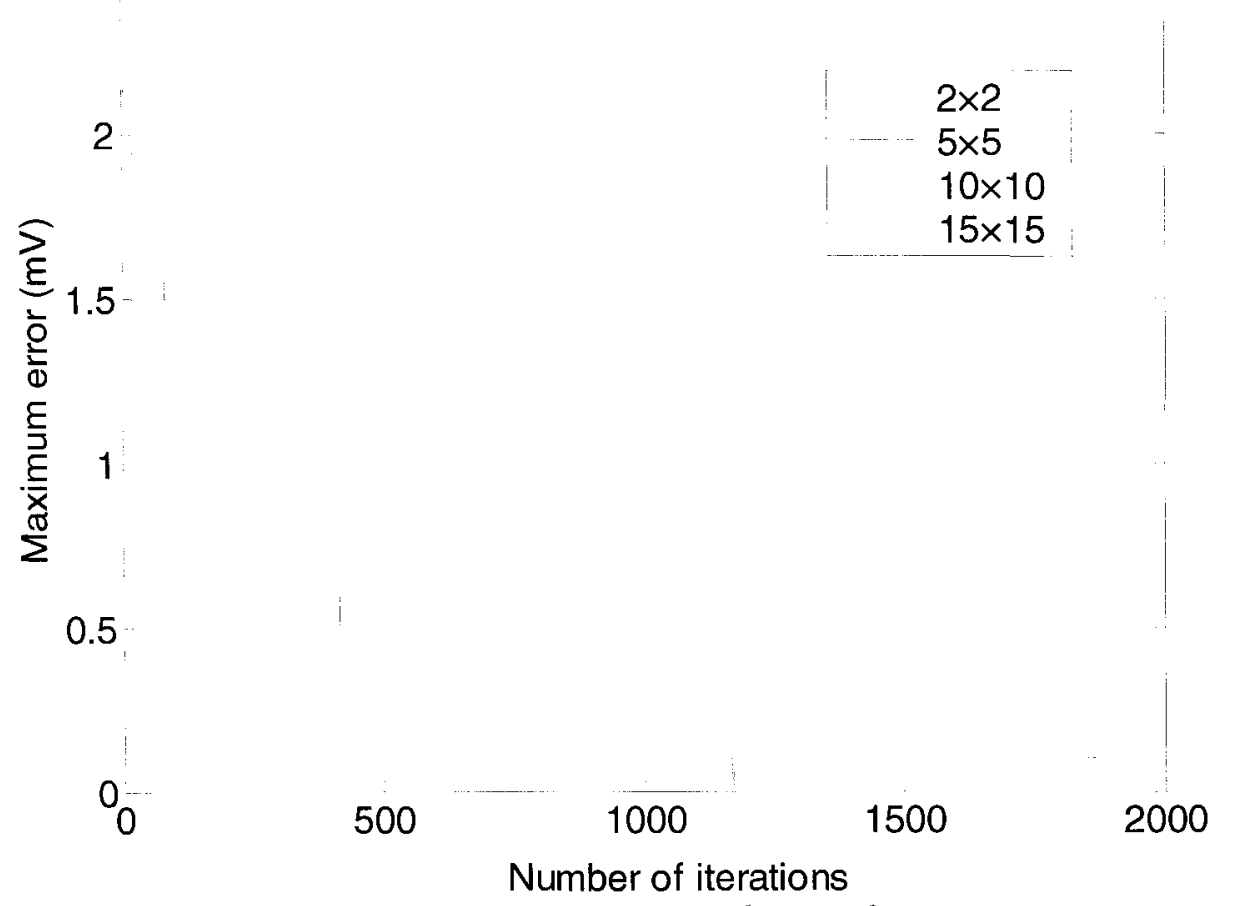

Fig. 5.40: Convergence process for $i_{L}=1 \times 10^{-6} \mathrm{~A} / \mathrm{mm}^{2}$ (Element size analysis)

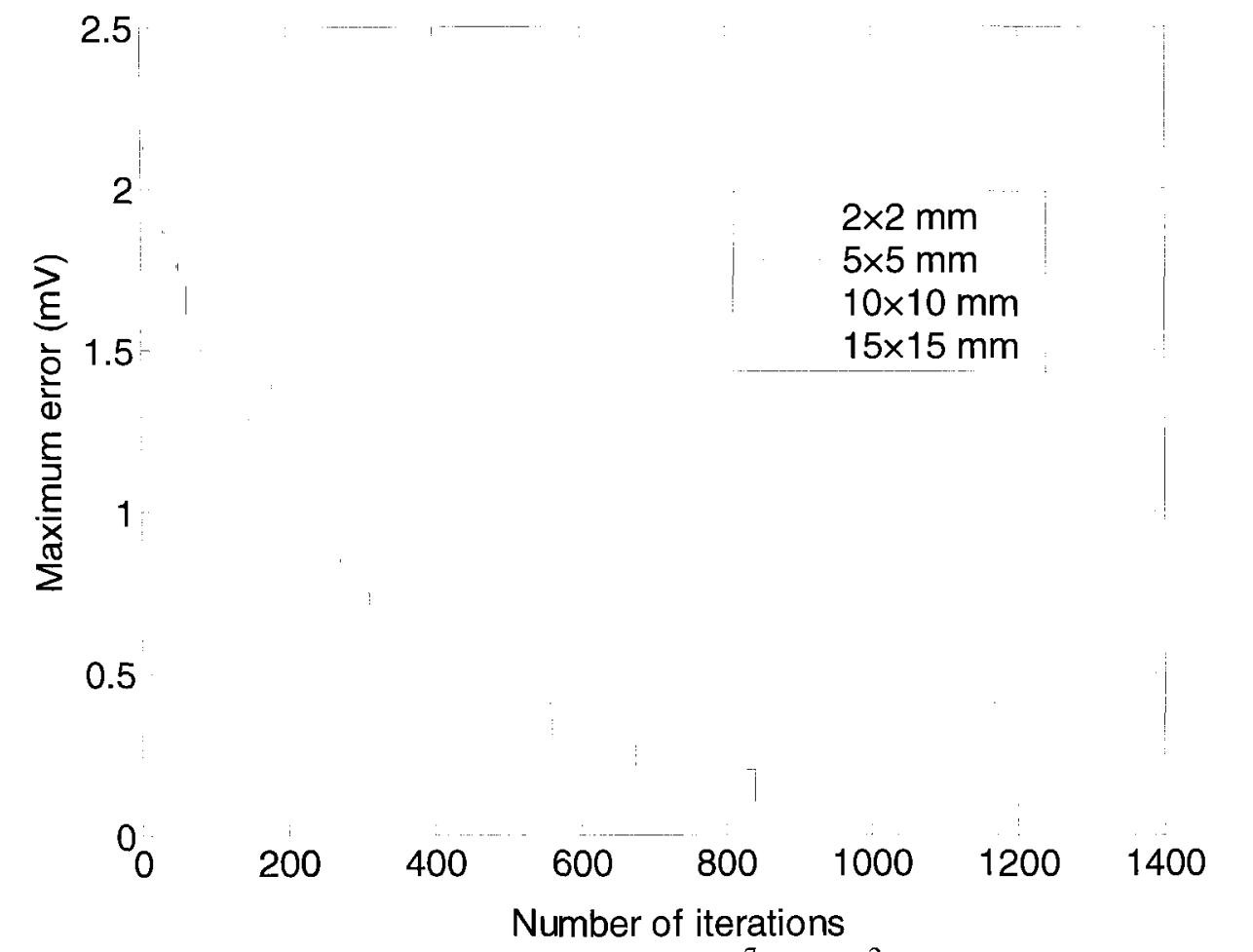

Fig. 5.41: Convergence process for $i_{L}=1 \times 10^{-7} \mathrm{~A} / \mathrm{mm}^{2}$ (Element size analysis) 


\section{5}

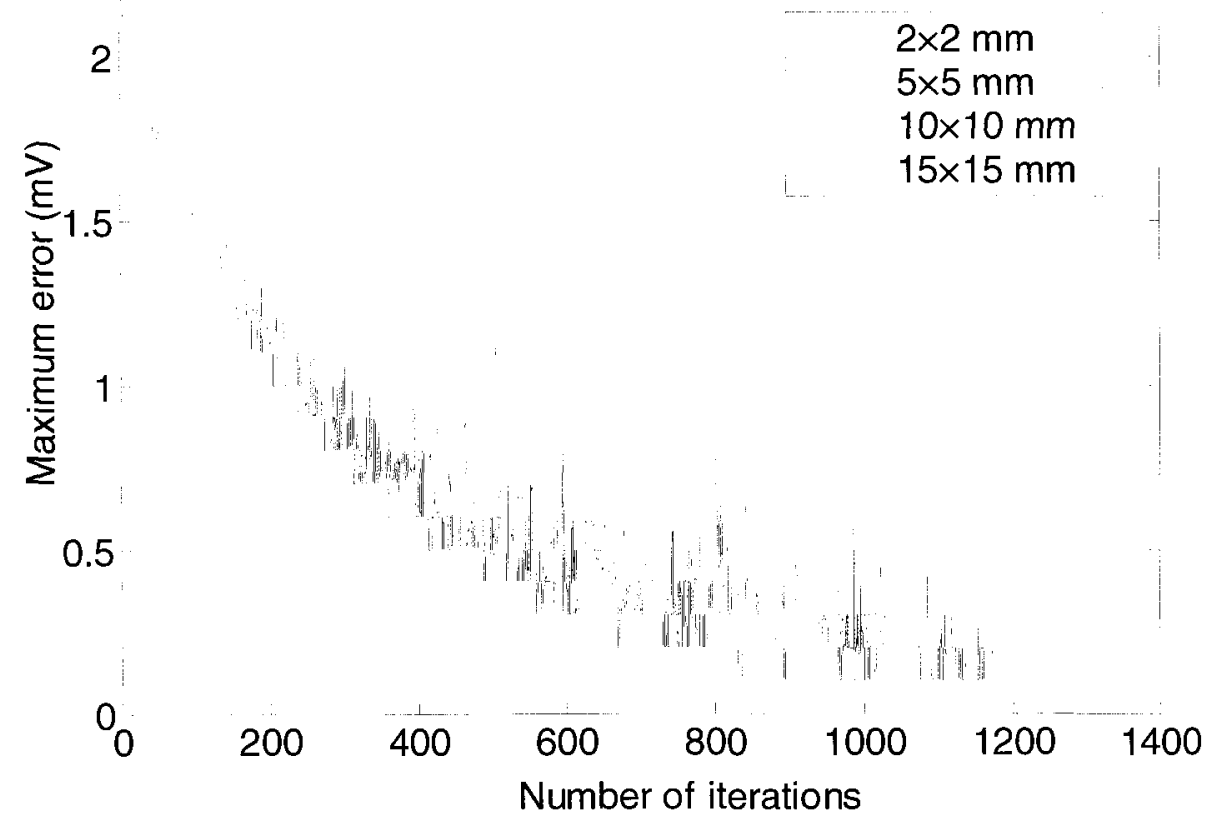

Fig. 5.42: Convergence process for $i_{L}=1 \times 10^{-8} \mathrm{~A} / \mathrm{mm}^{2}$ (Element size analysis)

\subsubsection{Effect of Anode-to-cathode ratio}

The effect of $\mathrm{A} / \mathrm{C}$ ratio on the convergence of the nonlinear solution of the base case is studied by varying $\mathrm{A} / \mathrm{C}$ ratio from 0.034 to 1.0 . Table 5.9 provides the input data and the results of the analysis. Figures 5.43-5.45, 5.46-5.48, 5.49-5.51, respectively illustrate the potential distribution, current density and convergence process for different limiting current densities.

As it can be observed from Figs. 5.43-5.48 that the numerical solution shows some fluctuations and inaccuracies as the $\mathrm{A} / \mathrm{C}$ ratio becomes very small (i.e $\mathrm{A} / \mathrm{C}=0.034$ and 0.071). The main reason for these inaccuracies is that for small $\mathrm{A} / \mathrm{C}$ ratios, the anode 
length is relatively small, and the element size used for these cases is not small enough to capture the effect of the anode. For example, for $\mathrm{A} / \mathrm{C}=0.034$, the anode length is $10 \mathrm{~mm}$; hence only two 5-mm elements represent the anode. In order to eliminate the fluctuations that are observed in Figs. 5.43-5.48, elements smaller than $5 \mathrm{~mm}$ must be used, however, as it can be observed in the previous section, this leads to convergence problems due to size effect. Therefore, the investigation of the solution of very small $\mathrm{A} / \mathrm{C}$ ratios requires further investigation, which is not in the scope of this research.

On the other hand, the MDIM is quite successful in solving $\mathrm{A} / \mathrm{C}$ ratio larger than 0.07 for all limiting current densities. The potential tend to decrease on the steel surface as the A/C ratio becomes larger; however since this change is mostly uniform for both anodes and cathodes for cases with high limiting current density (Cases 1-14), the current density is not affected significantly. For cases with low limiting current density (Cases 15-21), as shown in Figs. 5.45 and 5.48, potential difference between the anodes and cathodes slightly increase, and this leads to higher current densities as $\mathrm{A} / \mathrm{C}$ ratio becomes smaller.

As illustrated in Fig. 5.49-5.51, if the cases with $\mathrm{A} / \mathrm{C}=0.034$ and $\mathrm{A} / \mathrm{C}=0.071$ are ignored, $\mathrm{A} / \mathrm{C}$ ratio does not have an effect on the corrosion rate. The limiting current density factors used in cases with low limiting current densities prevent divergence; and their effect can be easily seen from Fig. 5.51. 
Table 5.9: A/C ratio analysis data and results by the MDIM

\begin{tabular}{|c|c|c|c|c|c|c|c|c|c|}
\hline Case & $\begin{array}{c}\text { Element } \\
\text { size } \\
(\mathrm{mm})\end{array}$ & $\begin{array}{c}r \\
(o h m-m m)\end{array}$ & $\mathrm{A} / \mathrm{C}$ & $\begin{array}{c}i_{L} \\
\left(\mathbf{A} / \mathbf{m m}^{2}\right)\end{array}$ & $\xi$ & $\psi_{1}{ }^{(1)}$ & $\psi_{2}^{(1)}$ & $\begin{array}{c}\text { Max. } \\
\text { Error }^{(2)} \\
(\mathbf{m V})\end{array}$ & $\begin{array}{l}\text { Corresponding } \\
\text { iteration }^{(3)}\end{array}$ \\
\hline 1 & 5 & 140000 & 0.034 & $10^{-6}$ & 0.003 & N/A & N/A & $<0.1$ & 1240 \\
\hline 2 & 5 & 140000 & 0.071 & $10^{-6}$ & 0.003 & N/A & N/A & $<0.1$ & 2082 \\
\hline 3 & 5 & 140000 & 0.111 & $10^{-6}$ & 0.003 & N/A & N/A & $<0.1$ & 1199 \\
\hline 4 & 5 & 140000 & 0.25 & $10^{-6}$ & 0.003 & N/A & N/A & $<0.1$ & 1173 \\
\hline 5 & 5 & 140000 & 0.5 & $10^{-6}$ & 0.003 & N/A & N/A & $<0.1$ & 1182 \\
\hline 6 & 5 & 140000 & 0.764 & $10^{-6}$ & 0.003 & N/A & N/A & $<0.1$ & 1192 \\
\hline 7 & 5 & 140000 & 1 & $10^{-6}$ & 0.003 & N/A & N/A & $<0.1$ & 1190 \\
\hline 8 & 5 & 140000 & 0.034 & $10^{-7}$ & 0.003 & N/A & $\mathrm{N} / \mathrm{A}$ & $<0.1$ & 1232 \\
\hline 9 & 5 & 140000 & 0.071 & $10^{-7}$ & 0.003 & N/A & $\mathrm{N} / \mathrm{A}$ & $<0.1$ & 1465 \\
\hline 10 & 5 & 140000 & 0.111 & $10^{-7}$ & 0.003 & N/A & N/A & $<0.1$ & 1203 \\
\hline 11 & 5 & 140000 & 0.25 & $10^{-7}$ & 0.003 & N/A & N/A & $<0.1$ & 1191 \\
\hline 12 & 5 & 140000 & 0.5 & $10^{-7}$ & 0.003 & N/A & N/A & $<0.1$ & 1191 \\
\hline 13 & 5 & 140000 & 0.764 & $10^{-7}$ & 0.003 & N/A & N/A & $<0.1$ & 1200 \\
\hline 14 & 5 & 140000 & 1 & $10^{-7}$ & 0.003 & N/A & N/A & $<0.1$ & 1195 \\
\hline 15 & 5 & 140000 & 0.034 & $10^{-8}$ & 0.003 & 0.9999 & 0.992 & 0.1 & 1199 \\
\hline 16 & 5 & 140000 & 0.071 & $10^{-8}$ & 0.003 & 0.9999 & 0.992 & 0.1 & 1028 \\
\hline 17 & 5 & 140000 & 0.111 & $10^{-8}$ & 0.003 & 0.9999 & 0.992 & $<0.1$ & 1196 \\
\hline 18 & 5 & 140000 & 0.25 & $10^{-8}$ & 0.003 & 0.9999 & 0.992 & $<0.1$ & 1201 \\
\hline 19 & 5 & 140000 & 0.5 & $10^{-8}$ & 0.003 & 0.9999 & 0.992 & $<0.1$ & 1204 \\
\hline 20 & 5 & 140000 & 0.764 & $10^{-8}$ & 0.003 & 0.9999 & 0.992 & $<0.1$ & 1194 \\
\hline 21 & 5 & 140000 & 1 & $10^{-8}$ & 0.003 & 0.9999 & 0.992 & $<0.1$ & 1248 \\
\hline
\end{tabular}

(1) Two factors are used when there is a plateau in potential distribution on steel surface

(2) Smallest value of the maximum error after 5000 iterations

(3) The iteration corresponding to the smallest value of the maximum error 


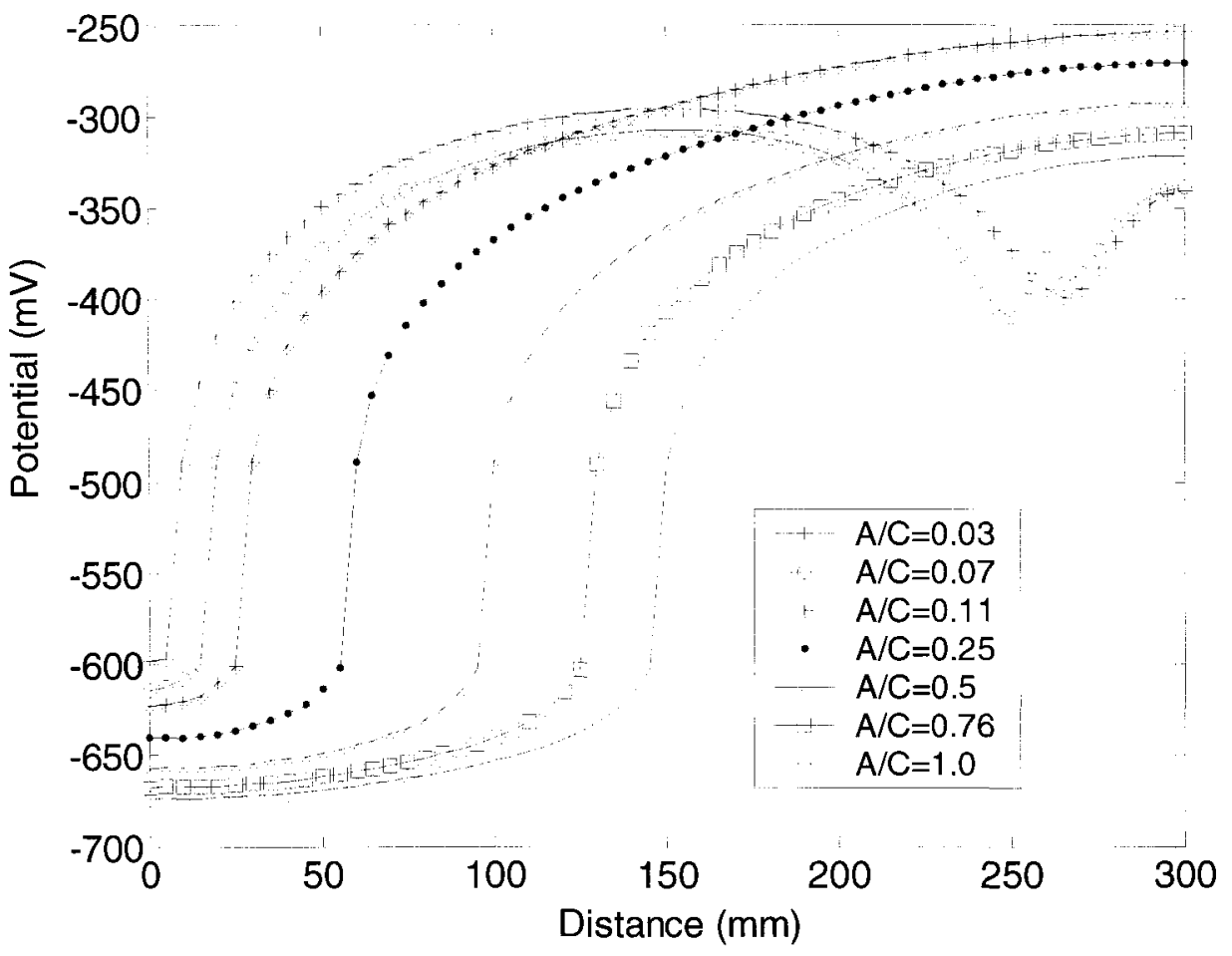

Fig. 5.43: Potential distribution on the rebar for $i_{L}=1 \times 10^{-6} \mathrm{~A} / \mathrm{mm}^{2}$ (A/C analysis)

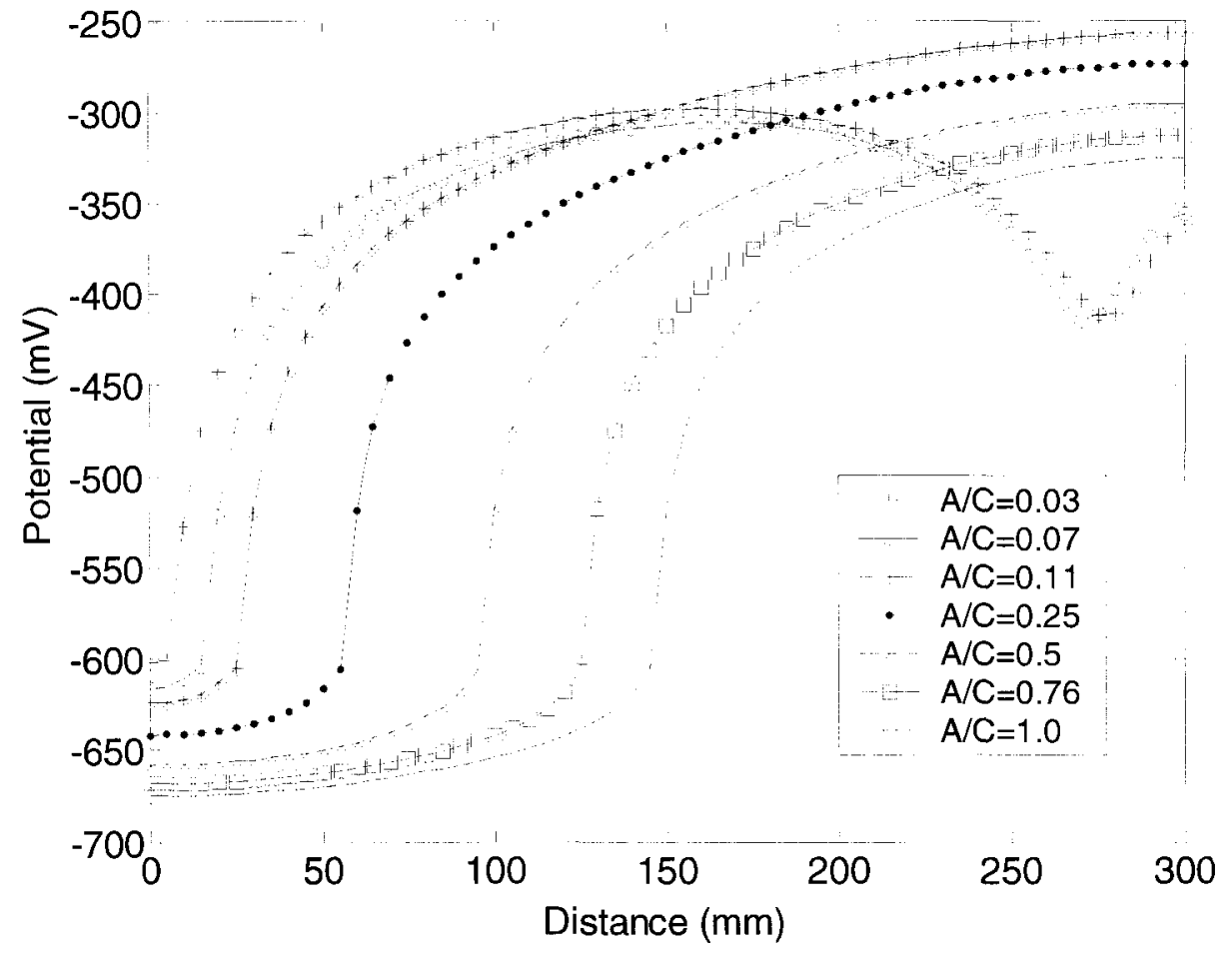

Fig. 5.44: Potential distribution on the rebar for $i_{L}=1 \times 10^{-7} \mathrm{~A} / \mathrm{mm}^{2}$ (A/C analysis) 


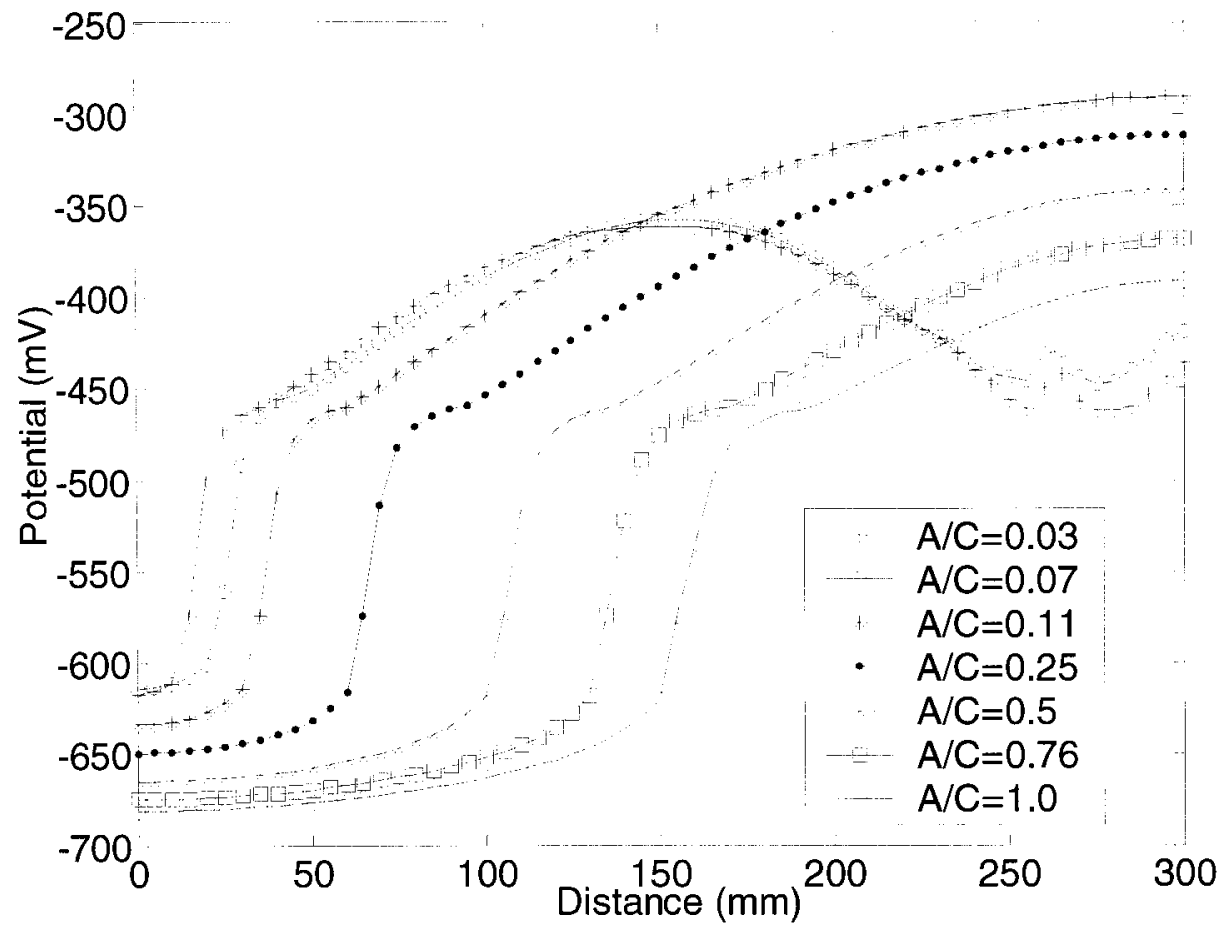

Fig. 5.45: Potential distribution on the rebar for $i_{L}=1 \times 10^{-8} \mathrm{~A} / \mathrm{mm}^{2}$ (A/C analysis)

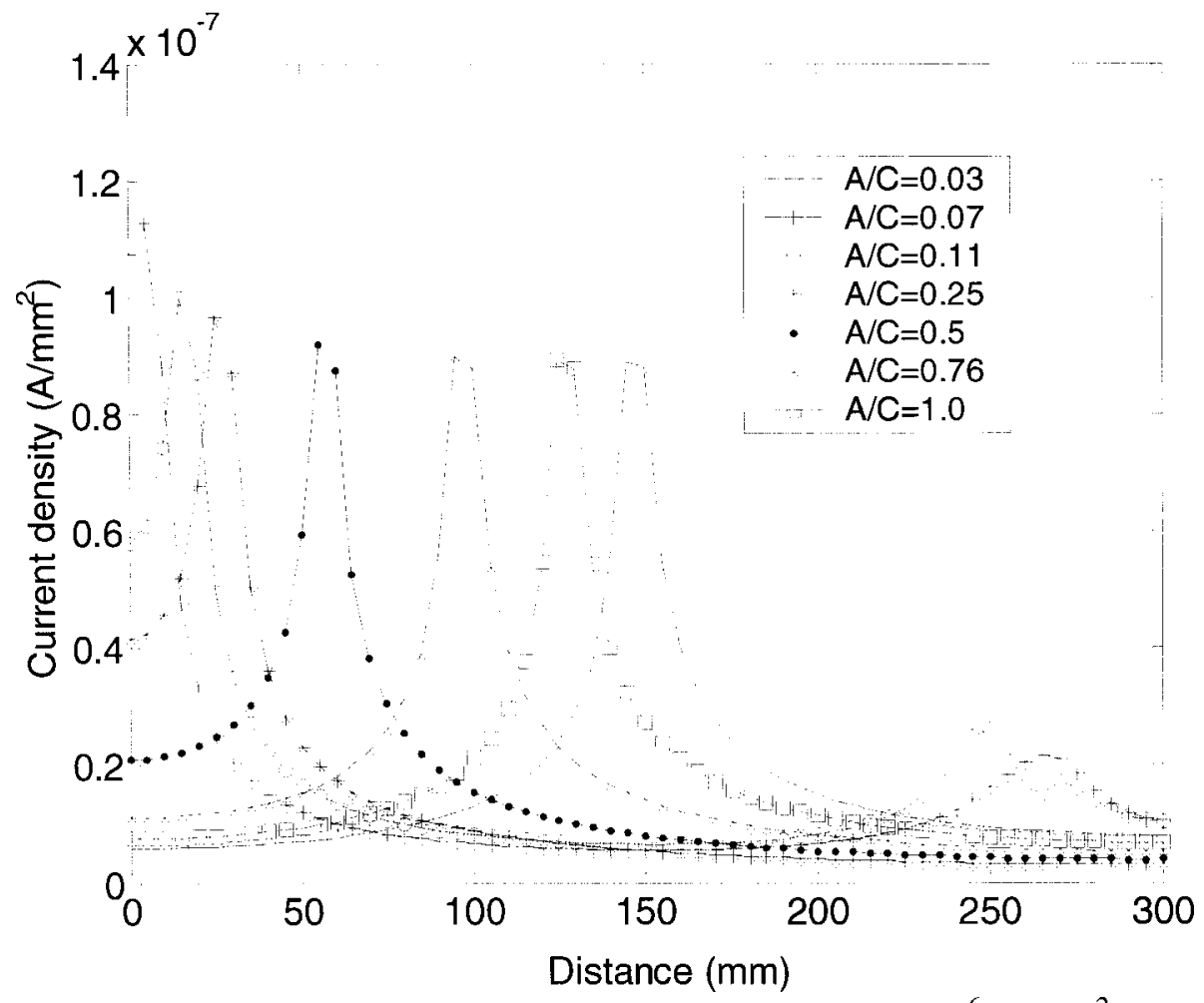

Fig. 5.46: Current density distribution on the rebar for $i_{L}=1 \times 10^{-6} \mathrm{~A} / \mathrm{mm}^{2}$ (A/C analysis) 


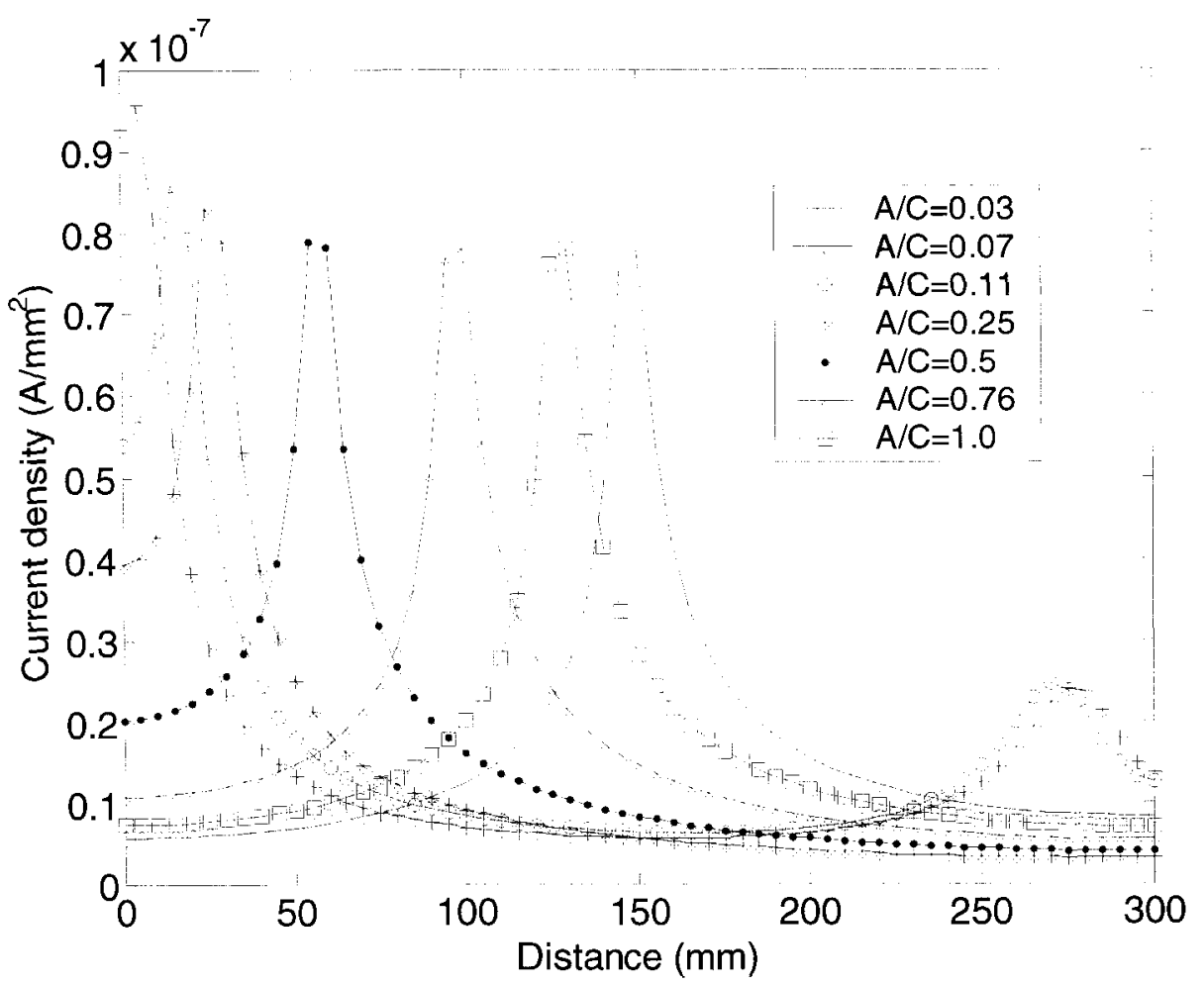

Fig. 5.47: Current density distribution on the rebar for $i_{L}=1 \times 10^{-7} \mathrm{~A} / \mathrm{mm}^{2}$ ( $\mathrm{A} / \mathrm{C}$ analysis)

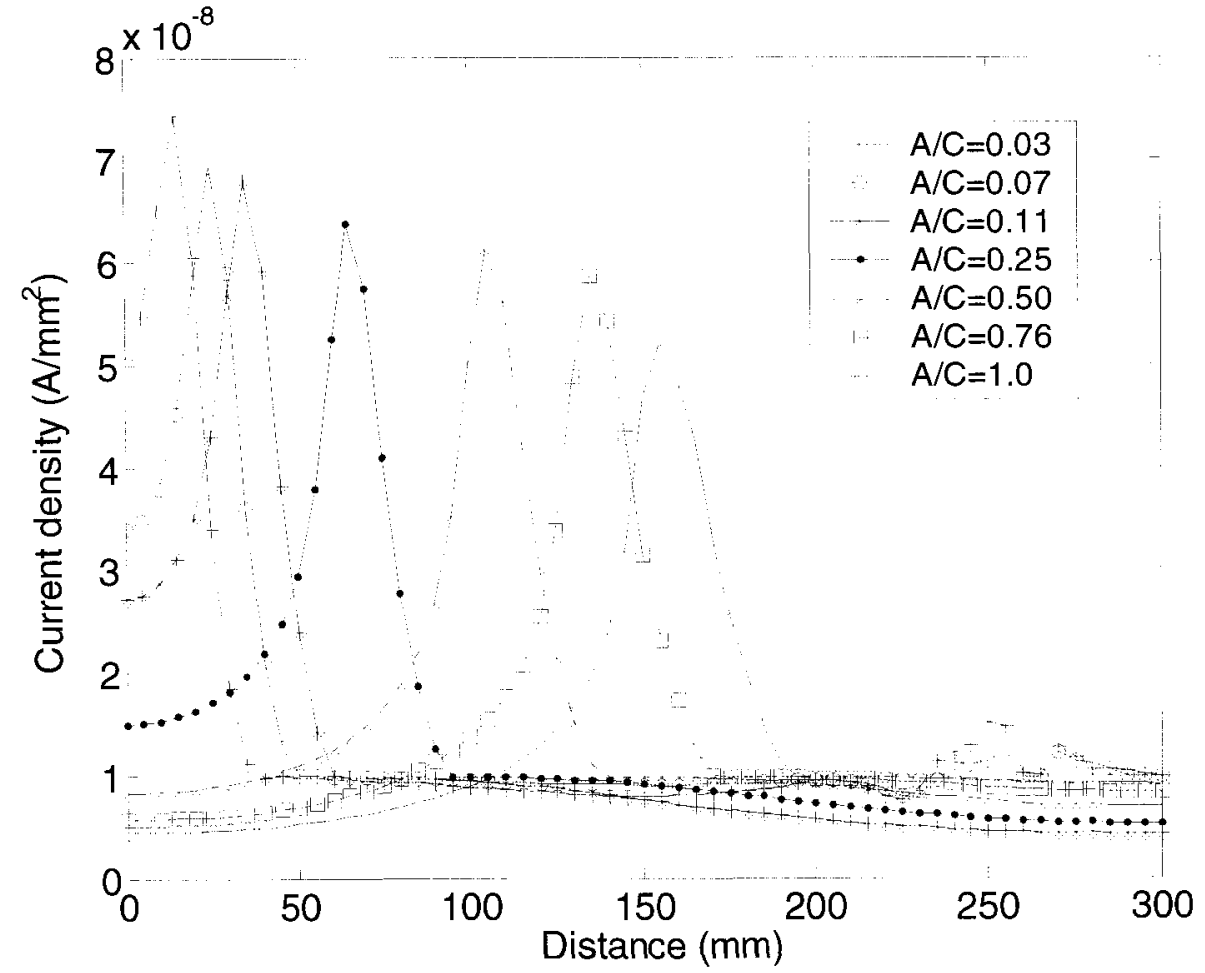

Fig. 5.48: Current density distribution on the rebar for $i_{L}=1 \times 10^{-8} \mathrm{~A} / \mathrm{mm}^{2}$ (A/C analysis) 
2.5

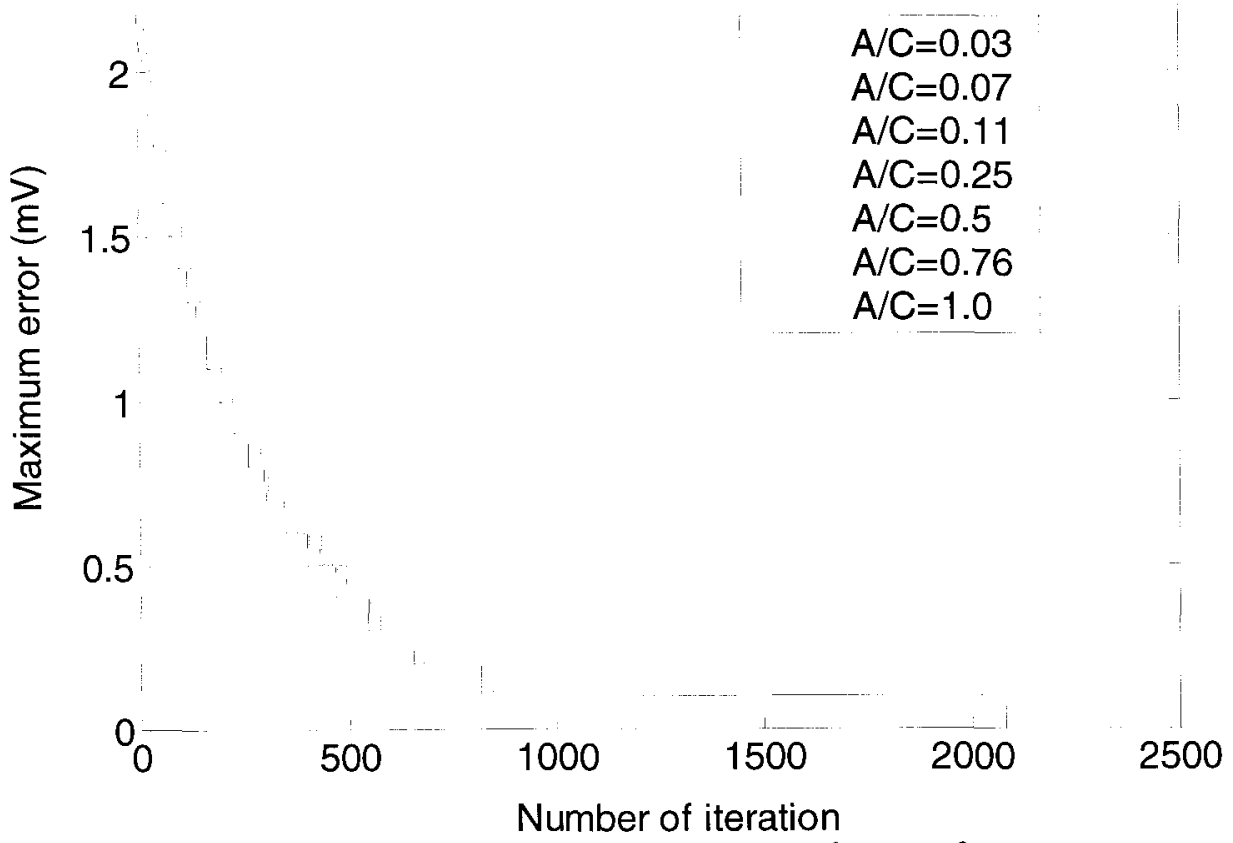

Fig. 5.49: Convergence process for $i_{L}=1 \times 10^{-6} \mathrm{~A} / \mathrm{mm}^{2}$ (A/C analysis)

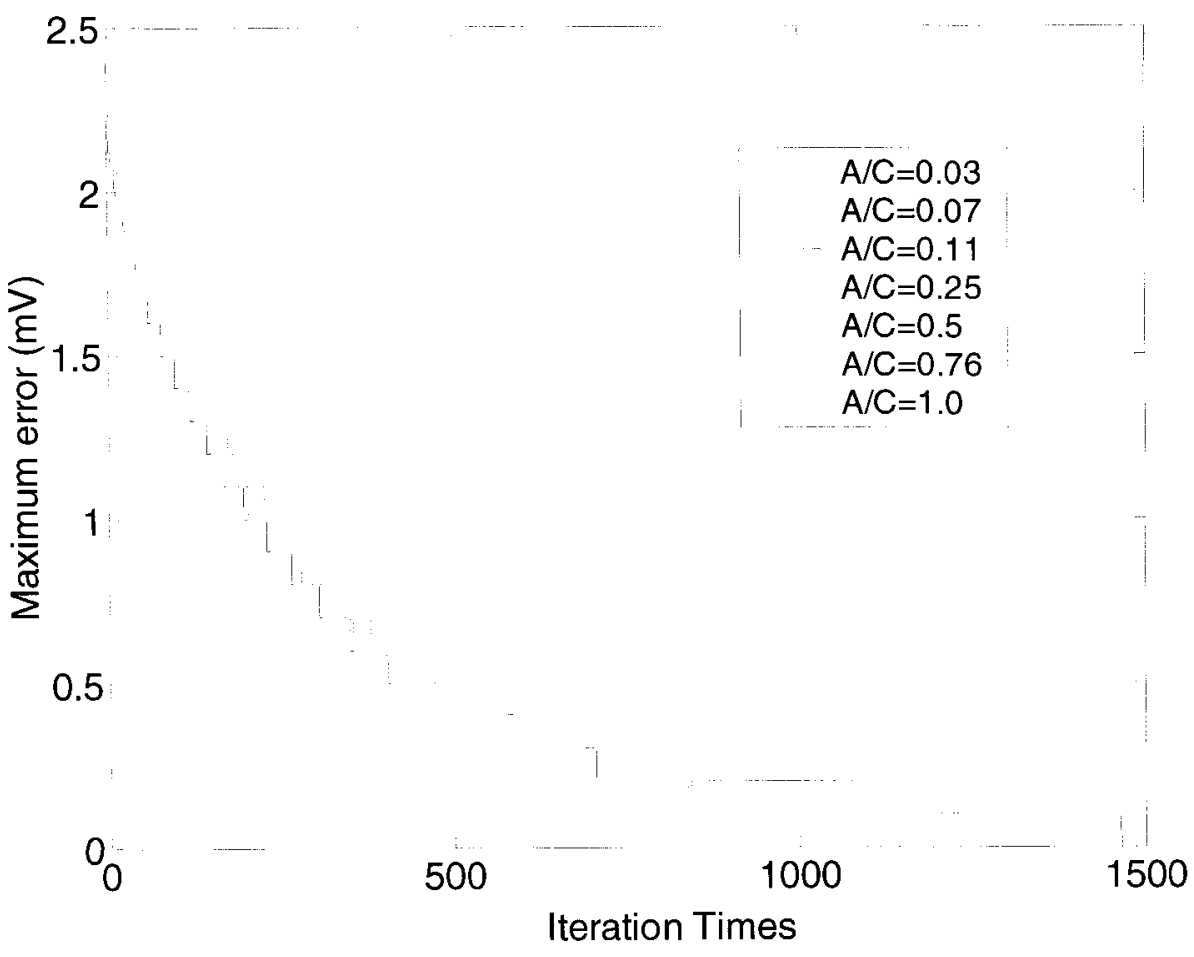

Fig. 5.50: Convergence process for $i_{L}=1 \times 10^{-7} \mathrm{~A} / \mathrm{mm}^{2}$ (A/C analysis) 


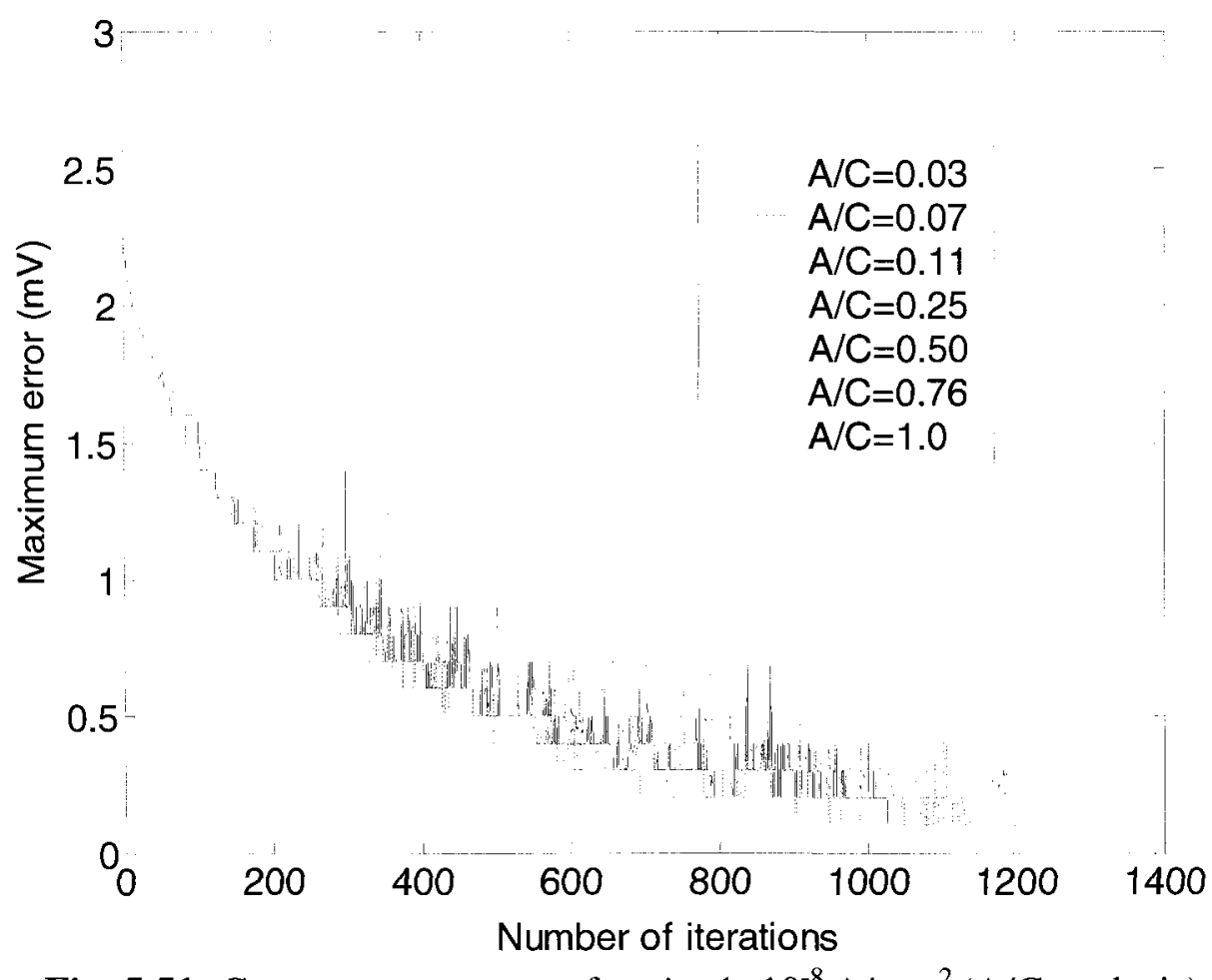

Fig. 5.51: Convergence process for $i_{L}=1 \times 10^{-8} \mathrm{~A} / \mathrm{mm}^{2}$ (A/C analysis)

\subsubsection{Effect of resistivity}

The effect of concrete resistivity on the convergence of the nonlinear solution of the base case is studied by varying concrete resistivity from 25,000 to $10,000,000 \mathrm{Ohm}-\mathrm{mm}$. Table 5.10 provides the input data and the results of the analysis. Figures 5.52-5.54, 5.55-5.57, 5.58-5.60, respectively illustrate the potential distribution, current density and convergence process for different limiting current densities.

It can be observed from Figs. 5.52-5.55 that resistivity has significant effect on the results and on the convergence rate. As illustrated in Figs. 5.52-5.54, the potential difference between anode and cathode becomes larger as the resistivity increases; however, the 
current density (see Figs. 5.55-57) decreases with increasing resistivity, even though the potential gradient becomes larger. This is an important observation explaining why potential mapping (e.g. half cell potential measurements) cannot be used as an indicator of corrosion rate in a reinforced concrete structures.

Concrete resistivity also affects the convergence process. As shown in Fig. 5.58, the convergence curve is not smooth for $r=25000 \mathrm{ohm}-\mathrm{mm}$. As the resistivity becomes smaller (i.e. more conductive concrete), it is observed that achieving convergence is harder. This can be explained by Eq. 3.6, in which current density is inversely proportional to concrete resistivity. As resistivity decreases, the nodal values of current density become large, and in some cases, these values may become larger than limiting current density. 
Table 5.10: Resistivity analysis data and results by the MDIM

\begin{tabular}{|c|c|c|c|c|c|c|c|c|c|}
\hline Case & $\begin{array}{c}\text { Element } \\
\text { size } \\
(\mathrm{mm})\end{array}$ & $\begin{array}{c}r \\
(o h m-m m)\end{array}$ & $\mathrm{A} / \mathrm{C}$ & $\left(\begin{array}{c}i_{L} \\
\left(\mathrm{~A} / \mathbf{m m}^{2}\right)\end{array}\right)$ & $\xi$ & $\psi_{1}^{(1)}$ & $\psi_{2}^{(1)}$ & $\begin{array}{c}\text { Max. } \\
\text { Error }^{(2)} \\
(\mathbf{m V})\end{array}$ & $\begin{array}{c}\text { Corresponding } \\
\text { iteration }^{(3)}\end{array}$ \\
\hline 1 & 5 & 25000 & 0.111 & $10^{-6}$ & 0.003 & N/A & N/A & $<0.1$ & 1201 \\
\hline 2 & 5 & 50000 & 0.111 & $10^{-6}$ & 0.003 & N/A & N/A & $<0.1$ & 1196 \\
\hline 3 & 5 & 100000 & 0.111 & $10^{-6}$ & 0.003 & N/A & N/A & $<0.1$ & 1199 \\
\hline 4 & 5 & 140000 & 0.111 & $10^{-6}$ & 0.003 & N/A & N/A & $<0.1$ & 1199 \\
\hline 5 & 5 & 500000 & 0.111 & $10^{-6}$ & 0.003 & N/A & N/A & $<0.1$ & 1177 \\
\hline 6 & 5 & 1000000 & 0.111 & $10^{-6}$ & 0.003 & N/A & N/A & $<0.1$ & 1171 \\
\hline 7 & 5 & 10000000 & 0.111 & $10^{-6}$ & 0.003 & N/A & N/A & $<0.1$ & 2147 \\
\hline 8 & 5 & 25000 & 0.111 & $10^{-7}$ & 0.003 & 0.9 & 0.8 & $<0.1$ & 1211 \\
\hline 9 & 5 & 50000 & 0.111 & $10^{-7}$ & 0.003 & 0.9 & 0.8 & $<0.1$ & 1203 \\
\hline 10 & 5 & 100000 & 0.111 & $10^{-7}$ & 0.003 & N/A & N/A & $<0.1$ & 1222 \\
\hline 11 & 5 & 140000 & 0.111 & $10^{-7}$ & 0.003 & N/A & N/A & $<0.1$ & 1208 \\
\hline 12 & 5 & 500000 & 0.111 & $10^{-7}$ & 0.003 & N/A & N/A & $<0.1$ & 1171 \\
\hline 13 & 5 & 1000000 & 0.111 & $10^{-7}$ & 0.003 & N/A & N/A & $<0.1$ & 1170 \\
\hline 14 & 5 & 10000000 & 0.111 & $10^{-7}$ & 0.003 & N/A & N/A & $<0.1$ & 2008 \\
\hline 15 & 5 & 25000 & 0.111 & $10^{-8}$ & 0.003 & 0.9999 & 0.999 & $<0.1$ & 1071 \\
\hline 16 & 5 & 50000 & 0.111 & $10^{-8}$ & 0.003 & 0.9999 & 0.995 & $<0.1$ & 1144 \\
\hline 17 & 5 & 100000 & 0.111 & $10^{-8}$ & 0.003 & 0.9999 & 0.99 & $<0.1$ & 1289 \\
\hline 18 & 5 & 140000 & 0.111 & $10^{-8}$ & 0.003 & 0.9999 & 0.99 & $<0.1$ & 1250 \\
\hline 19 & 5 & 500000 & 0.111 & $10^{-8}$ & 0.003 & 0.9 & 0.8 & $<0.1$ & 1173 \\
\hline 20 & 5 & 1000000 & 0.111 & $10^{-8}$ & 0.003 & 0.99 & 0.98 & $<0.1$ & 1212 \\
\hline 21 & 5 & 10000000 & 0.111 & $10^{-8}$ & 0.003 & N/A & N/A & $<0.1$ & 2042 \\
\hline
\end{tabular}

(1) Two factors are used when there is a plateau in potential distribution on steel surface

(2) Smallest value of the maximum error after 5000 iterations

(3) The iteration corresponding to the smallest value of the maximum error 


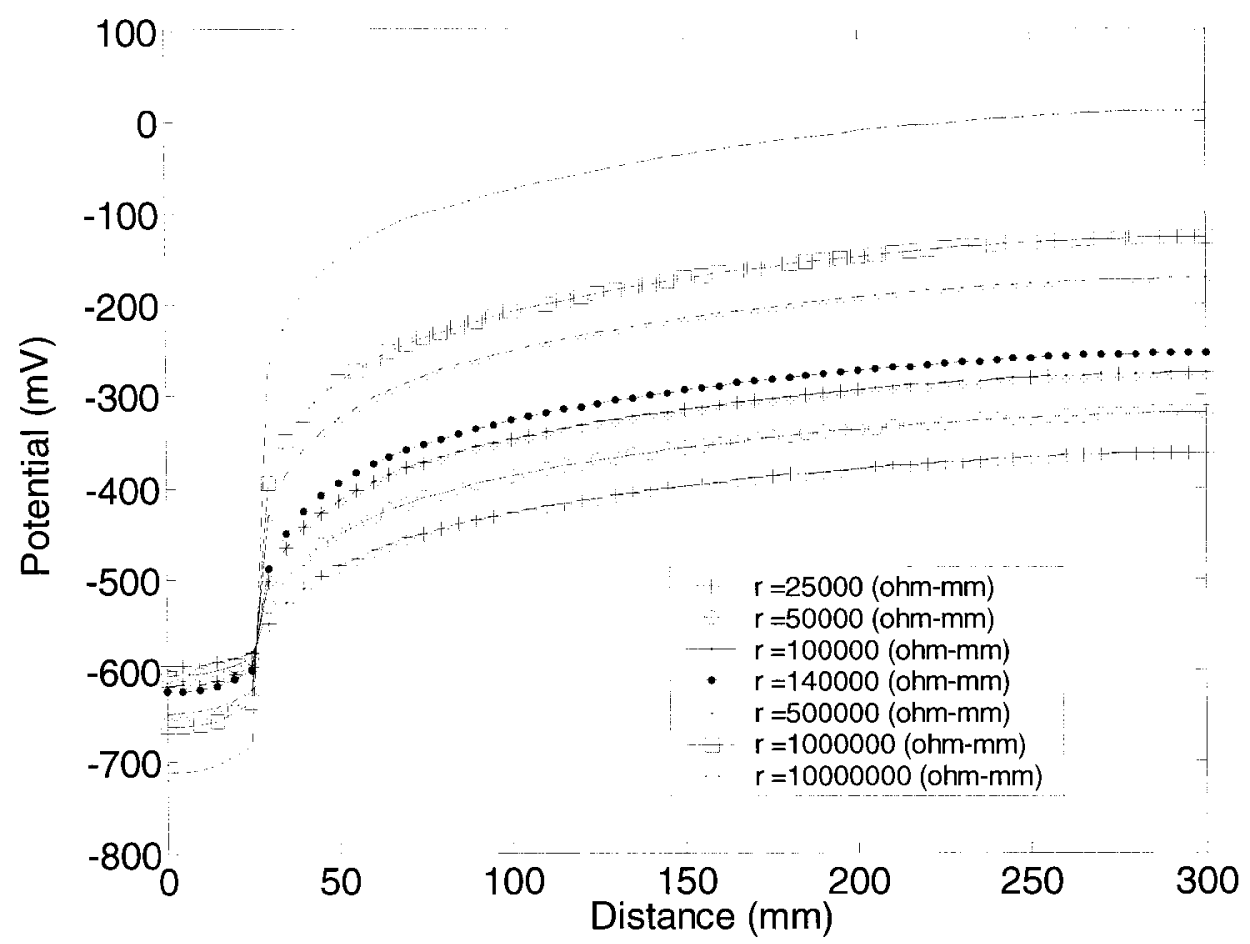

Fig. 5.52: Potential distribution on the rebar for $i_{L}=1 \times 10^{-6} \mathrm{~A} / \mathrm{mm}^{2}$ (Resistivity analysis)

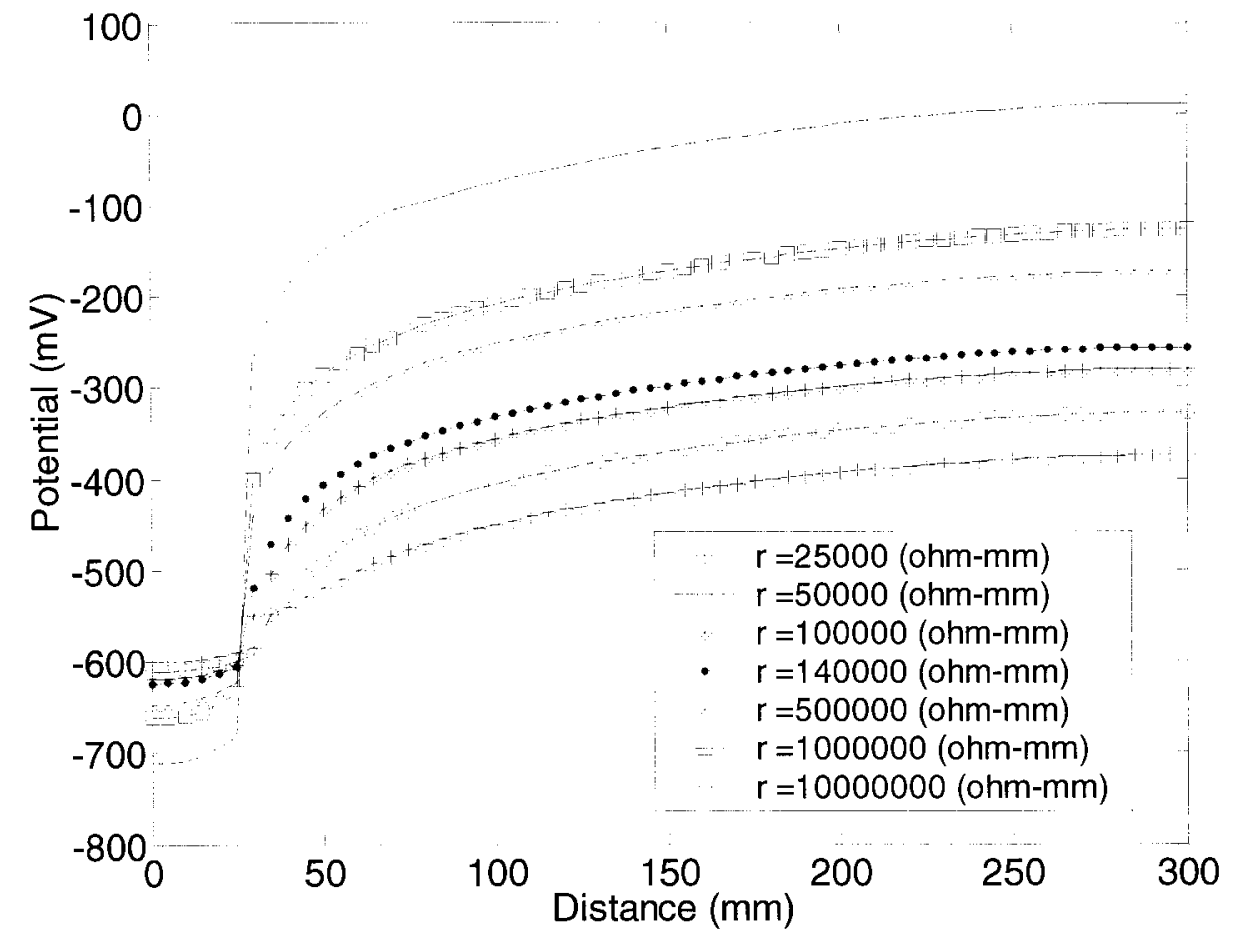

Fig. 5.53: Potential distribution on the rebar for $i_{L}=1 \times 10^{-7} \mathrm{~A} / \mathrm{mm}^{2}$ (Resistivity analysis) 


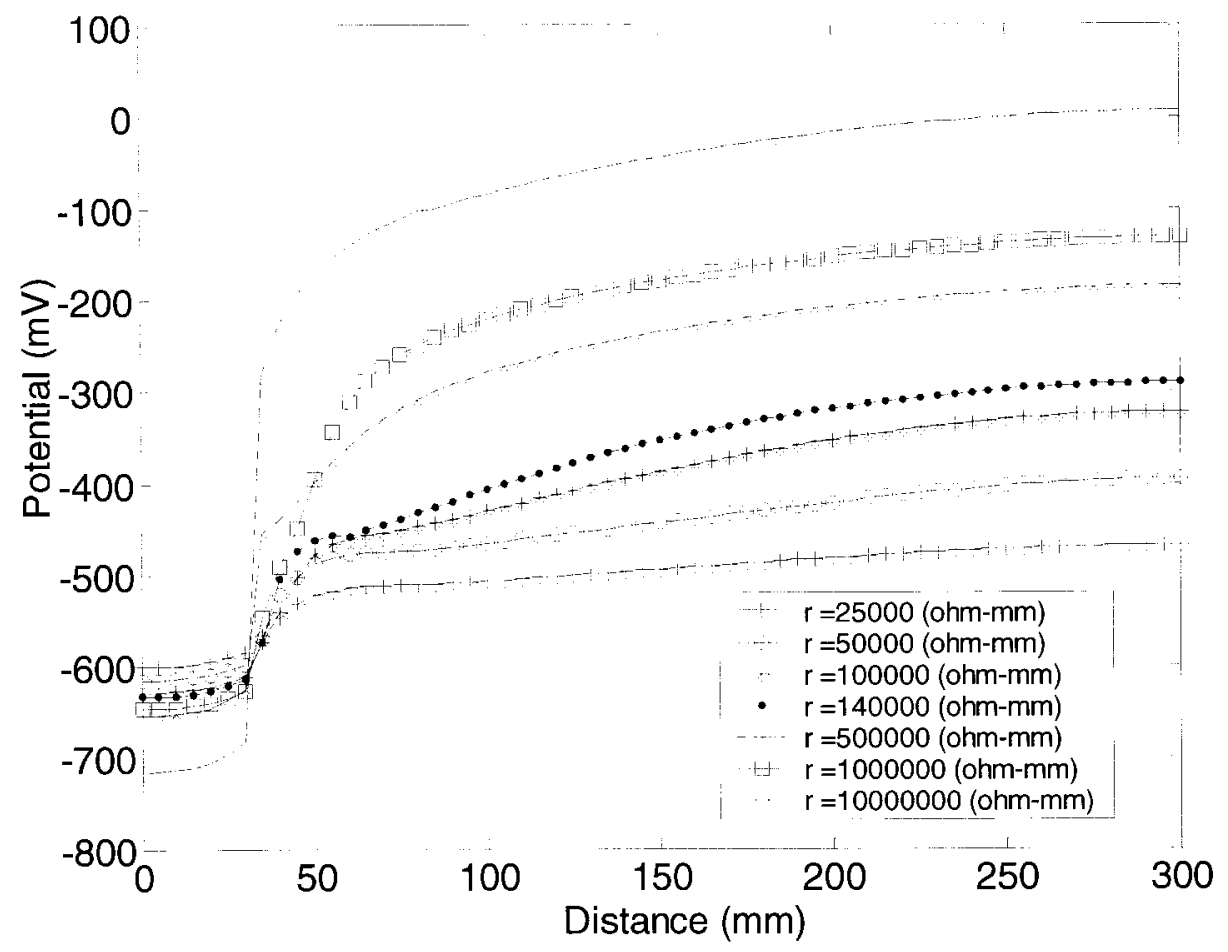

Fig. 5.54: Potential distribution on the rebar for $i_{L}=1 \times 10^{-8} \mathrm{~A} / \mathrm{mm}^{2}$ (Resistivity analysis)

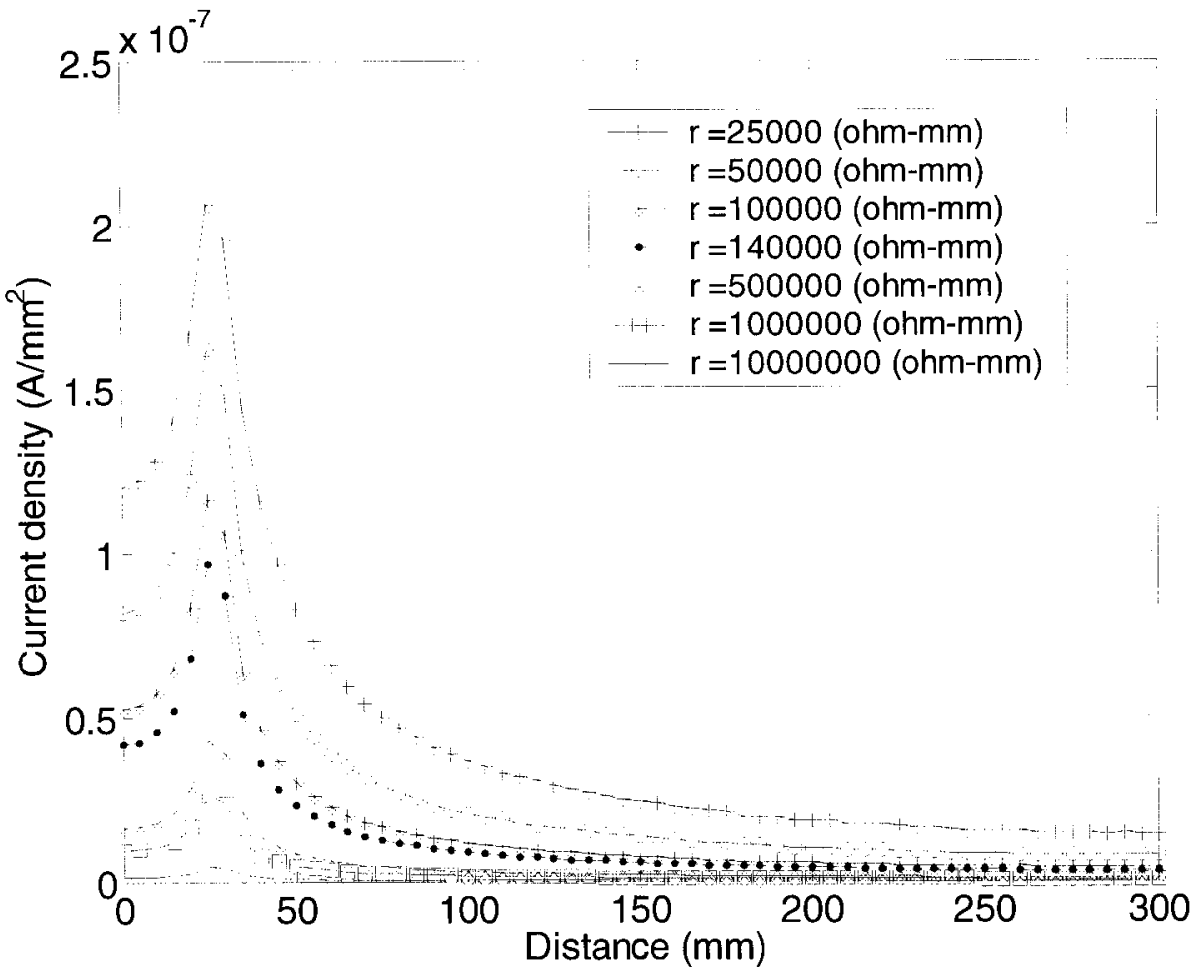

Fig. 5.55: Current density distribution on the rebar for $i_{L}=1 \times 10^{-6} \mathrm{~A} / \mathrm{mm}^{2}$ (Resistivity analysis) 


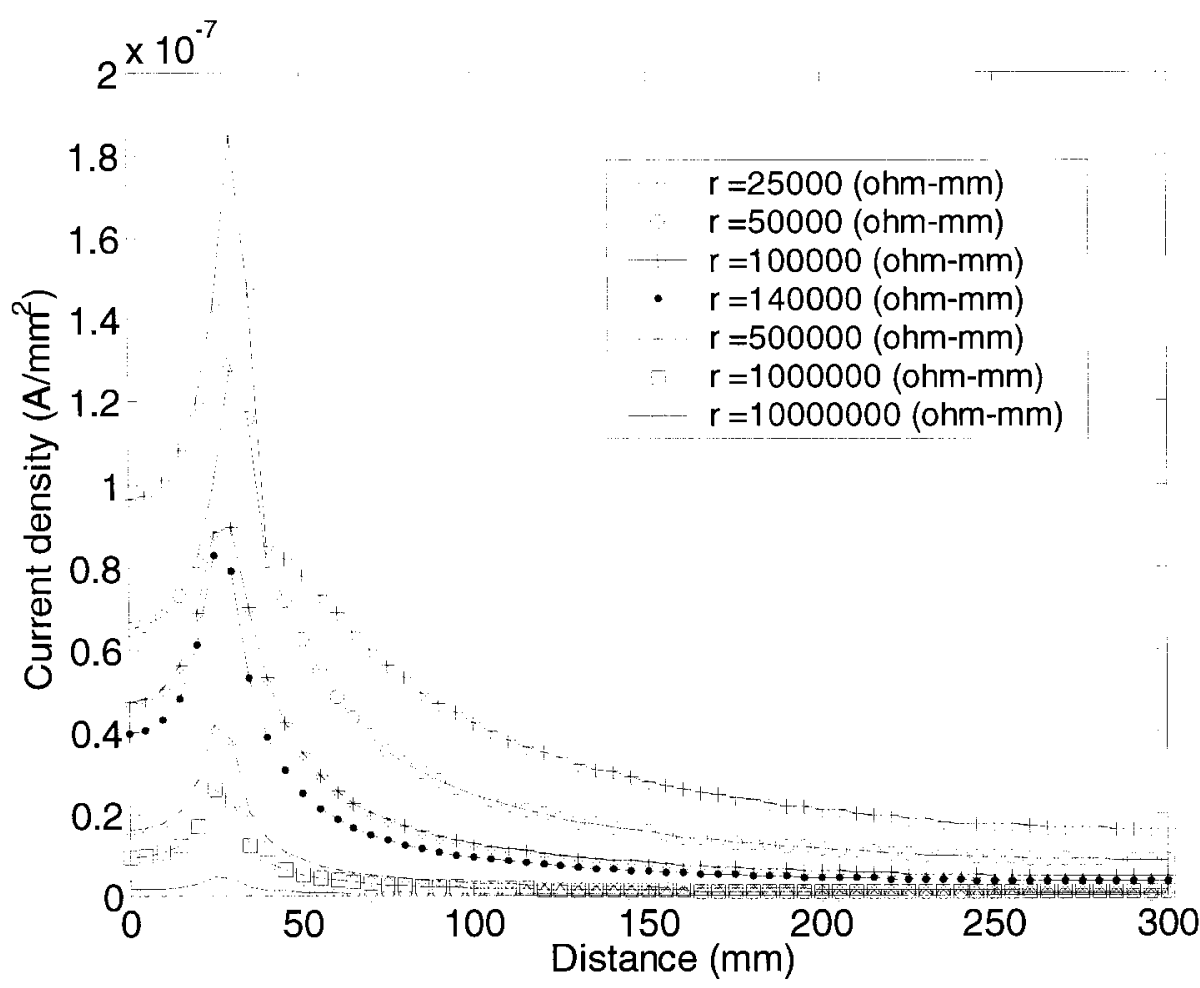

Fig. 5.56: Current density distribution on the rebar for $i_{L}=1 \times 10^{-7} \mathrm{~A} / \mathrm{mm}^{2}$ (Resistivity analysis)

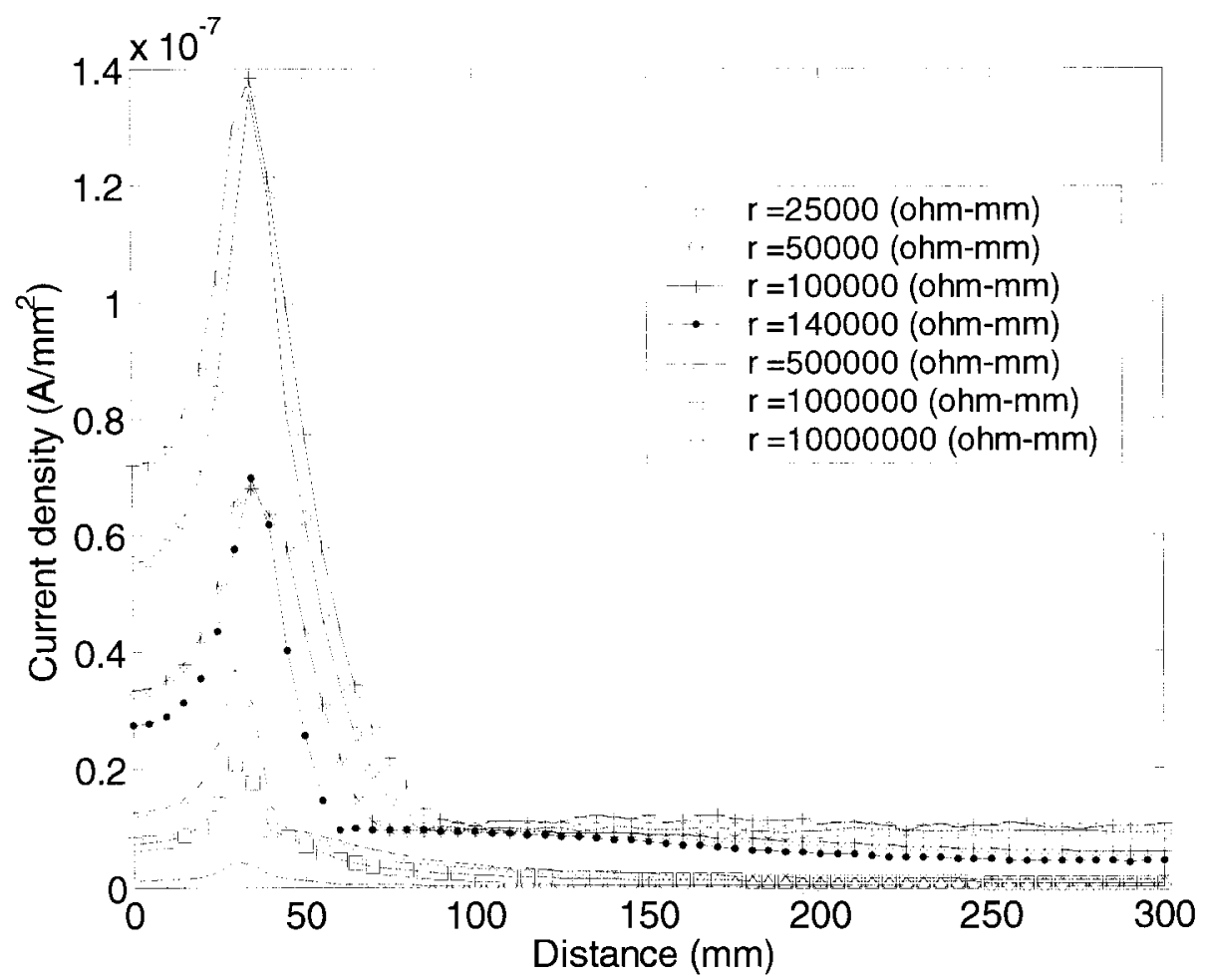

Fig. 5.57: Current density distribution on the rebar for $i_{L}=1 \times 10^{-8} \mathrm{~A} / \mathrm{mm}^{2}$ (Resistivity analysis) 


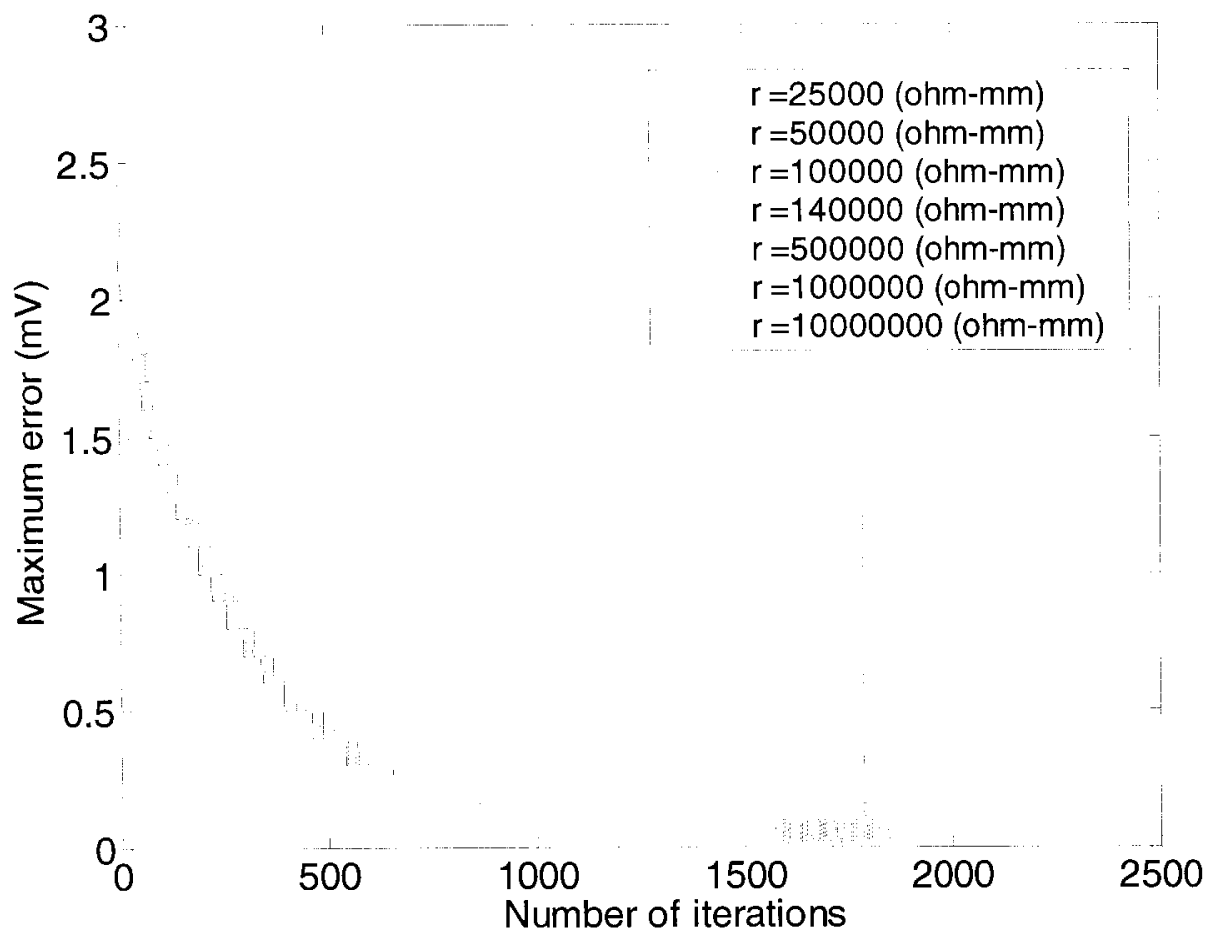

Fig. 5.58: Convergence process for $i_{L}=1 \times 10^{-6} \mathrm{~A} / \mathrm{mm}^{2}$ (Resistivity analysis)

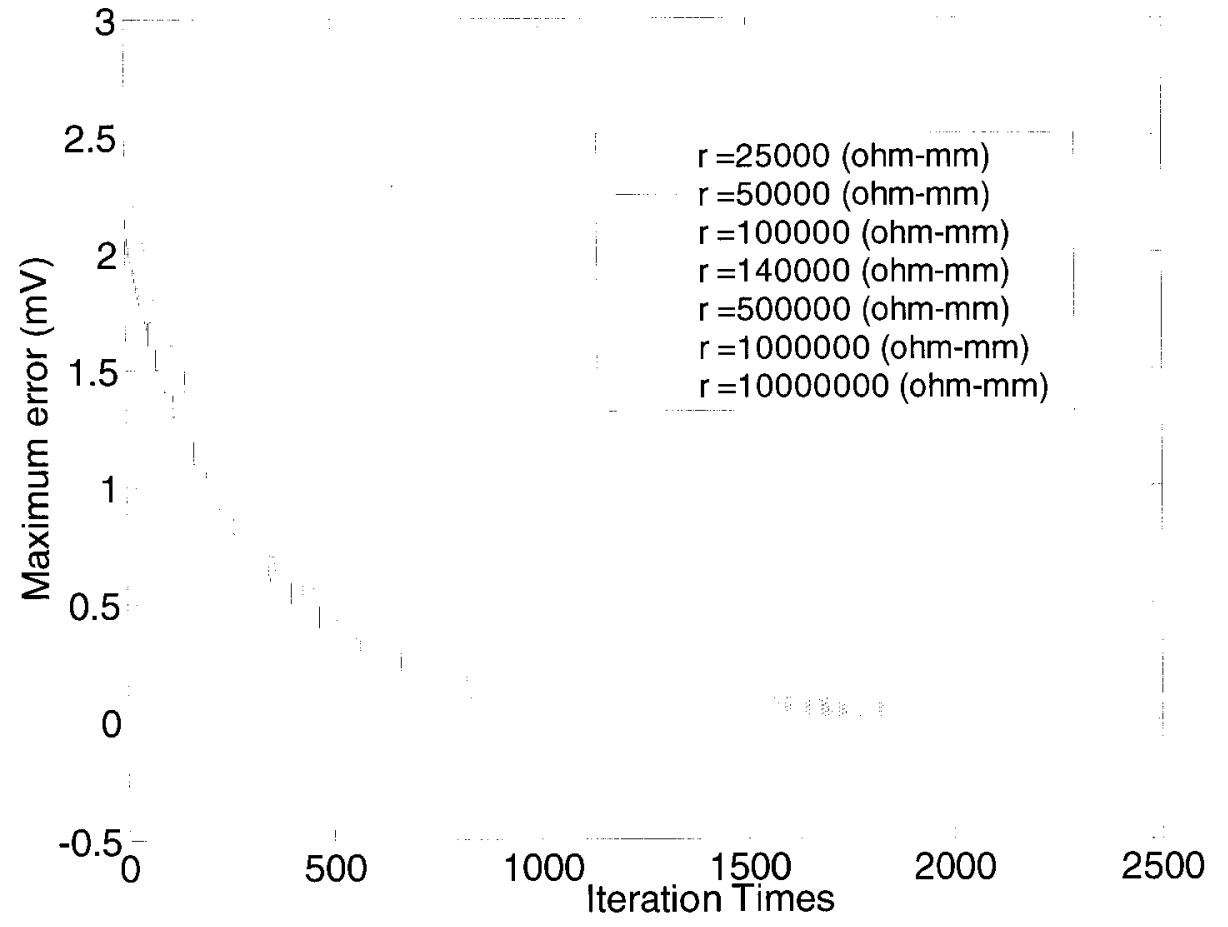

Fig. 5.59: Convergence process for $i_{L}=1 \times 10^{-7} \mathrm{~A} / \mathrm{mm}^{2}$ (Resistivity analysis) 


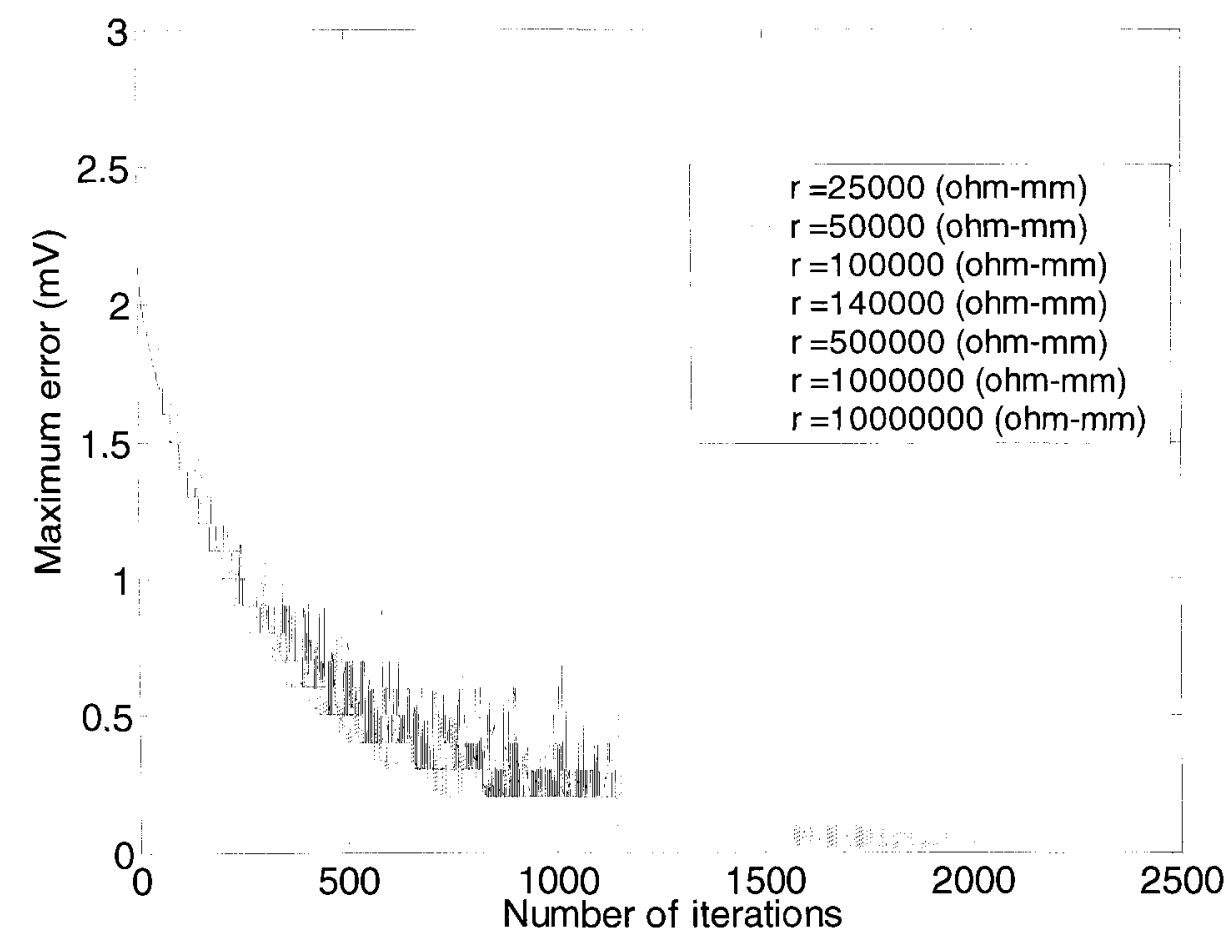

Fig. 5.60: Convergence process for $i_{L}=1 \times 10^{-8} \mathrm{~A} / \mathrm{mm}^{2}$ (Resistivity analysis)

\subsection{Summary of the numerical investigation}

The summary of the investigation presented in this chapter can be presented as follows:

- The DIM has limitations in solving the steel corrosion problem in concrete. As illustrated in Fig. 5.61, the convergence process in the DIM shows a random pattern, and the results obtained from the DIM show significant fluctuations.

- The issues with the DIM exist for all element sizes, $\mathrm{A} / \mathrm{C}$ ratios and concrete resistivities; therefore their origin is numerical.

- The MDIM, through under-relaxation and limiting current density control factors correct most issues encountered in the DIM. As illustrated in Fig. 5.61, the MDIM can provide smooth convergence and results when appropriate factors are selected. 
- For very small element sizes the MDIM show inaccuracies in results and convergence problems. For converged cases, the element size does not have a significant effect on convergence rate.

- In selecting the element size, a fine balance between the accuracy and convergence should be found.

- The numerical solution by the MDIM shows some fluctuations and inaccuracies as the $\mathrm{A} / \mathrm{C}$ ratio becomes very small (i.e $\mathrm{A} / \mathrm{C}=0.034$ and 0.071 ). The main reason for these inaccuracies is that for small $\mathrm{A} / \mathrm{C}$ ratios, the anode length is relatively small, and the element size used for these cases is not small enough to capture the effect of the anode. Therefore, the investigation of the solution of very small $\mathrm{A} / \mathrm{C}$ ratios requires further investigation.

- The MDIM is quite successful in solving $\mathrm{A} / \mathrm{C}$ ratios larger than 0.071 for all limiting current densities.

- If the cases with $\mathrm{A} / \mathrm{C}=0.034$ and $\mathrm{A} / \mathrm{C}=0.071$ are ignored, $\mathrm{A} / \mathrm{C}$ ratio does not have an effect on the corrosion rate.

- Concrete resistivity has significant effect on the results and on the convergence rate of the MDIM. The potential difference between anode and cathode becomes larger as the resistivity increases; however, the current density decreases with increasing resistivity, even though the potential gradient becomes larger. This is an important observation explaining why potential mapping (e.g. half cell potential measurements) may not be a good indicator of corrosion rate in a reinforced concrete structure.

- As concrete resistivity becomes smaller (i.e. more conductive concrete), it is observed that achieving convergence is harder. 

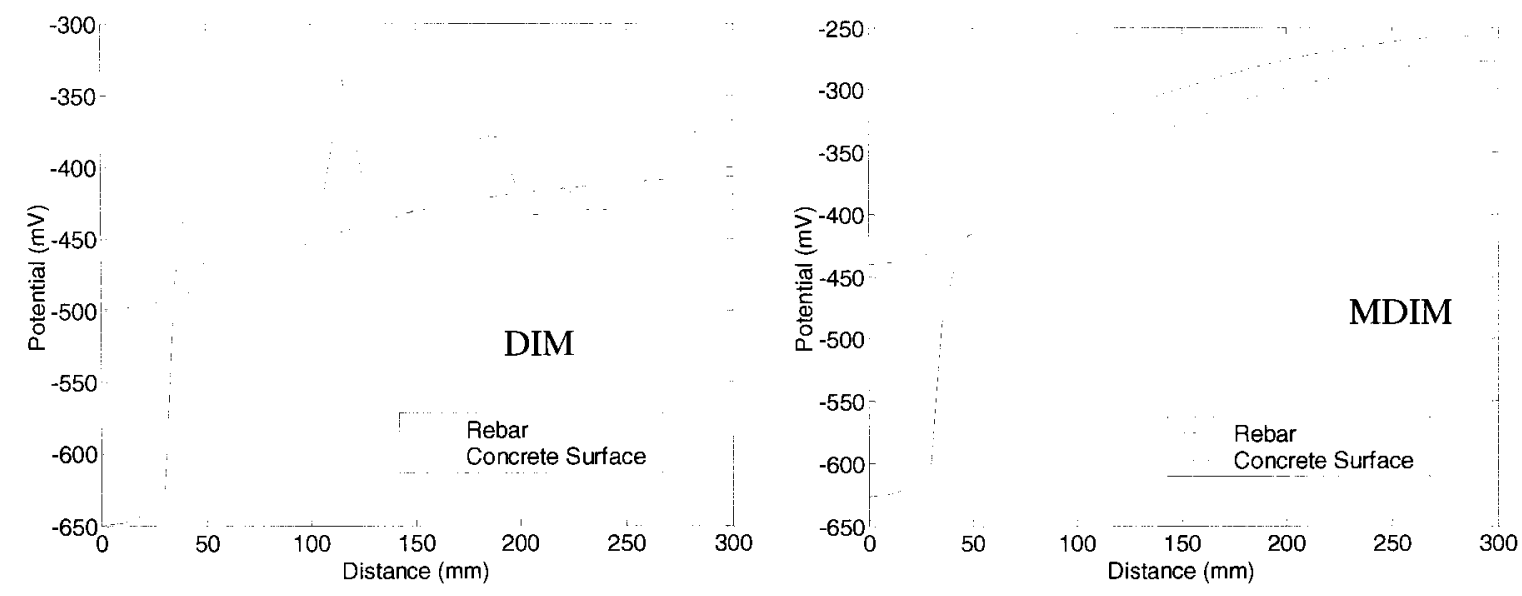

(a)
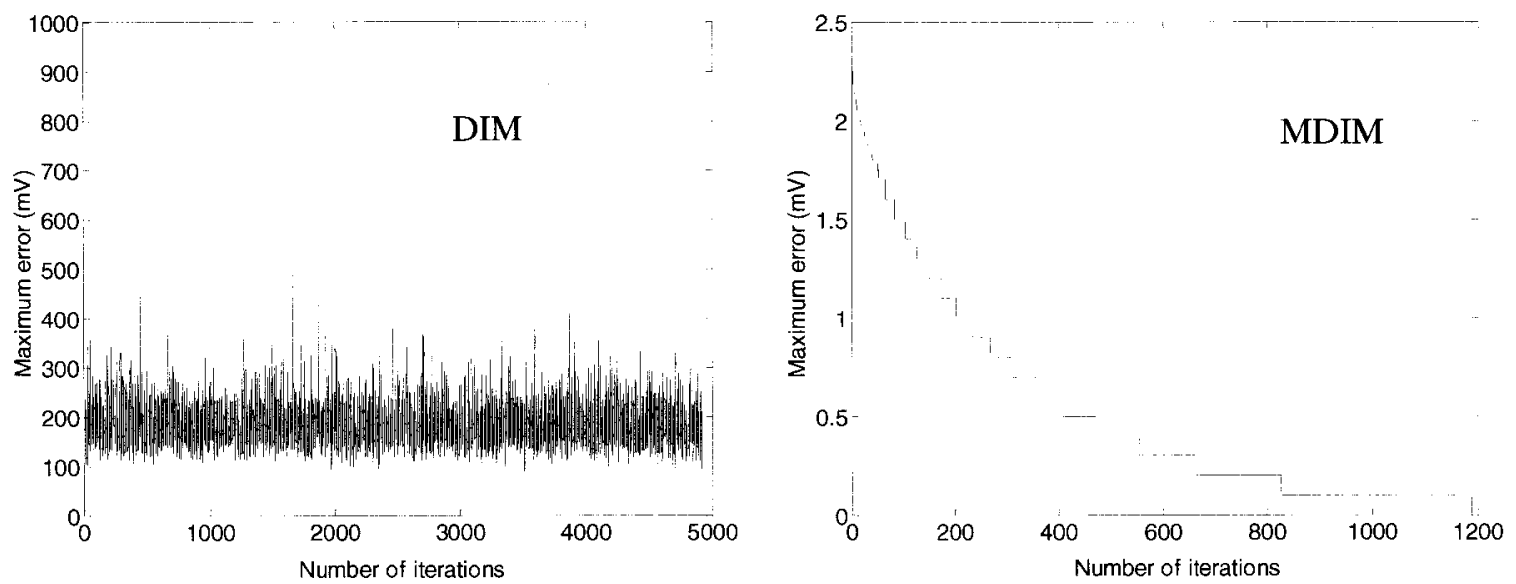

(b)

Fig. 5.61: Comparison of the DIM and the MDIM for the base case $\left(i_{L}=1 \times 10^{-6} \mathrm{~A} / \mathrm{mm}^{2}\right)$

(a) Potential distribution (b) Convergence process 


\section{Chapter 6}

\section{The Effect of Corrosion Parameters}

\subsection{General}

Besides the selection of element size, A/C ratio, concrete resistivity and limiting current density, the modelling of steel corrosion in concrete requires a number of input parameters be defined. These parameters (i.e. corrosion parameters), which originate from the basic corrosion theory, can be listed as follows:

- Anodic Tafel slope, $\beta_{a}$

- Cathodic Tafel slope, $\beta_{c}$

- Anodic exchange current density, $i_{o a}$

- Cathodic exchange current density, $i_{o c}$

- Standard electrode potential for the anodic reaction, $E_{o u}$

- Standard electrode potential for the cathodic reaction, $E_{o c}$

In the chapters presented so far, these quantities have been assumed to be constants that are selected within the range of reported values found in the corrosion literature. However, experimental studies have shown that these parameters can show significant variations; therefore the selection of these parameters for modelling purposes can be a challenging 
task. For instance, the anodic Tafel slope, $\beta_{a}$, and the cathodic Tafel slope $\beta_{c}$, have been reported to vary in a wide range between $40 \mathrm{mV}$ to $100 \mathrm{mV}$ and $120 \mathrm{mV}$ to $290 \mathrm{mV}$, respectively (Garces 2005). Environmental parameters, presence of external ions such as chlorides, etc. have also been shown to affect these parameters. For example, Tafel slopes decrease with increasing temperatures (Elsener 2005).

A parametric study is carried out to investigate the effect of variations in corrosion parameters on the rate of steel corrosion in concrete. The main goal of this investigation is to identify the parameters that have significant influence on corrosion rate. Since there is uncertainty associated with the section of these parameters, it is intended that the results of this study will provide valuable information to engineers who model steel corrosion in concrete. To achieve this goal, the effect of a single parameter on the corrosion rate is studied while all other parameters are kept constant. All simulations are run using the base case model that was described in Table 5.1. The parameter that is under investigation is varied while all other parameters are kept at their base-case values. For each parameter two extreme cases of $\mathrm{A} / \mathrm{C}$ ratio are studied: $\mathrm{A} / \mathrm{C}=0.111$ and $\mathrm{A} / \mathrm{C}=1.0$. The main reason behind this is the hypothesis that corrosion parameters may affect the corrosion rate differently at different $\mathrm{A} / \mathrm{C}$ ratios. It was assumed that there is adequate amount of oxygen around the steel; therefore the limiting current density, $i_{L}$, in all cases, is taken as $1 \times 10^{-6}$ $\mathrm{A} / \mathrm{mm}^{2}$, and the limited density correction factor is set to 1.0. An under-relaxation factor of $\xi=0.003$ is used. 


\subsection{Investigation of the anodic Tafel slope}

The effect of anodic Tafel slope on the corrosion rate of steel in concrete is studied by varying its value from $26 \mathrm{mV}$ to $91 \mathrm{mV}$. These limits represent the extreme boundary of possible anodic Tafel slopes that are observed in reinforced concrete structures. One of the steps in the range is $\beta_{a}=60 \mathrm{mV}$, which corresponds to the base case. The results of the analysis for $\mathrm{A} / \mathrm{C}=0.111$ and $\mathrm{A} / \mathrm{C}=1.0$ are shown in Figs. 6.1-6.3 and Figs. 6.4-6.6, respectively.

As illustrated in Figs. 6.1 and 6.2 , for $\mathrm{A} / \mathrm{C}=0.111$, the change in $\beta_{a}$ only affects potential and current density distribution along the anodic region. It can be observed in Fig. 6.1 that, as $\beta_{a}$ increases, the potential on the anodic steel surface also increases; however the potential distribution along the cathodic surface is not affected significantly. Therefore, as $\beta_{a}$ increases, the potential difference between the anodic and cathodic regions decrease, and this results in lower corrosion rates, as it can be observed in Fig. 6.2. The change in $\beta_{a}$ did not have a significant influence on the convergence rate of the numerical solution, as shown in Fig. 6.3. As illustrated in Figs. 6.4-6.6, similar qualitative observations can be made for $\mathrm{A} / \mathrm{C}=1.0$.

The change in corrosion rate vs. the change in anodic Tafel slope for both $\mathrm{A} / \mathrm{C}$ ratios is plotted in Fig. 6.7. The corrosion rate in this plot is taken as the average current density in the anodic region since this is a common practice for representing corrosion rate. Throughout this chapter, the change in corrosion rate is calculated using the following 
formula:

$$
\Delta i_{a, a v g}=\frac{\frac{1}{N_{a}} \sum_{n=1}^{N_{a}}\left(i_{n}-i_{n}^{b}\right)}{\frac{1}{N_{a}} \sum_{n=1}^{N_{a}}\left(i_{n}^{b}\right)}
$$

where $\Delta i_{a, a v g}$ is the change in corrosion rate, $N_{a}$ is the number of nodes along the anodic surface, $i_{n}$ is the calculated anodic current density at node $n$, and $i_{n}^{b}$ is the anodic current density at node $n$ for the base case.

As it can be seen from Fig. 6.7, the relationship between the two variations is linear with different slopes: For $\mathrm{A} / \mathrm{C}=0.111$ the slope is -0.51 and for $\mathrm{A} / \mathrm{C}=1.0$, the slope is -0.39 . The negative sign in front of the slopes signify that an increase in the anodic Tafel slope causes a reduction in the corrosion rate. The higher slope in $\mathrm{A} / \mathrm{C}=0.111$ shows that anodic Tafel slope has a more significant effect on the corrosion rate as the $\mathrm{A} / \mathrm{C}$ ratio becomes smaller. However, it should also be noted that the absolute values of slopes for both $\mathrm{A} / \mathrm{C}$ ratios are smaller than 1.0 , which means that the effect of a certain amount of change in anodic Tafel slope will cause less change in corrosion rate. For example, as it can be observed in Fig. 6.7, a $10 \%$ deviation in $\beta_{a}$ from the base case results in $5.1 \%$ and $3.4 \%$ change in corrosion rate for steel with $\mathrm{A} / \mathrm{C}=0.111$ and $\mathrm{A} / \mathrm{C}=1.0$, respectively. 


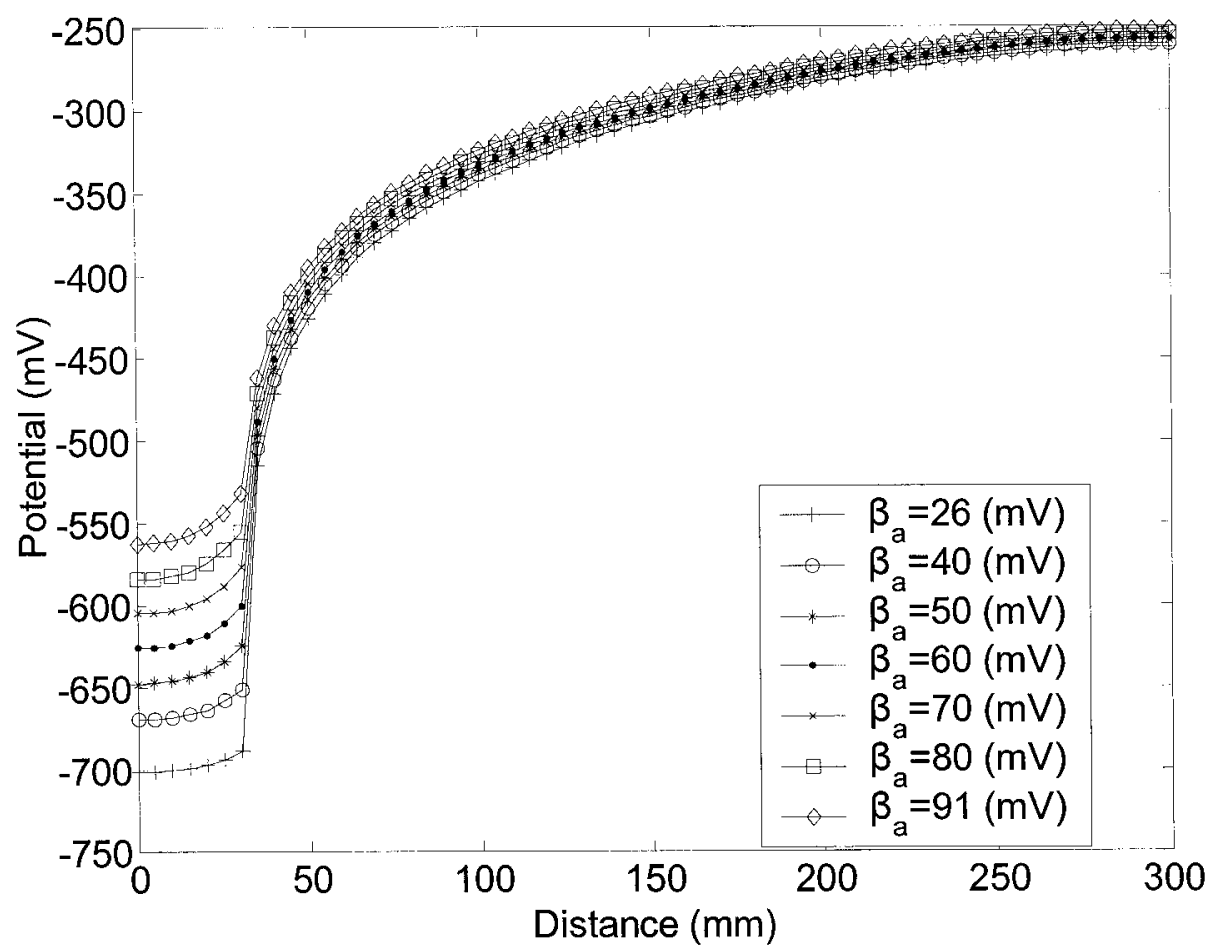

Fig. 6.1: The effect of anodic Tafel slope on potential distribution $(\mathrm{A} / \mathrm{C}=0.111)$

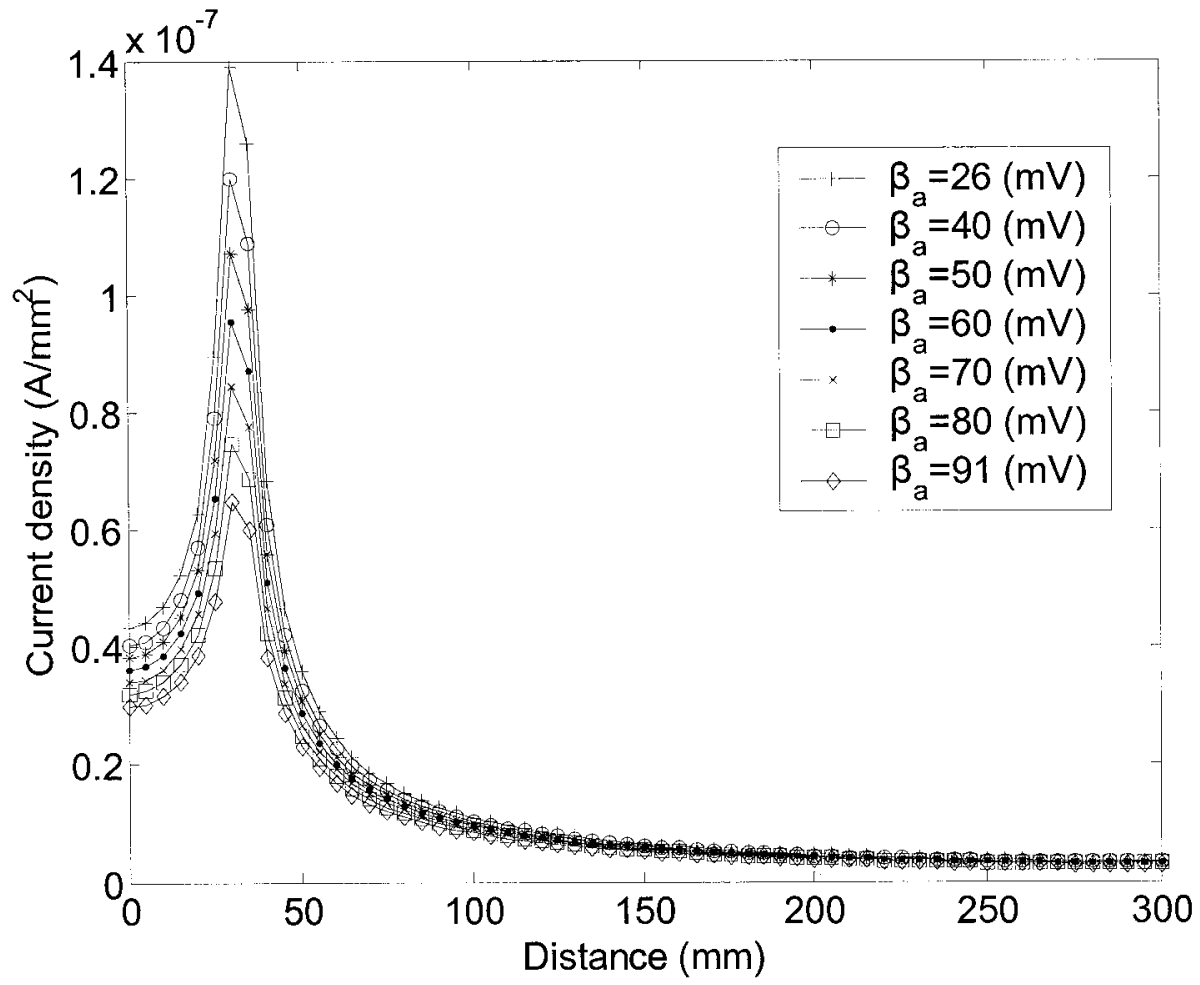

Fig. 6.2: The effect of anodic Tafel slope on current density $(\mathrm{A} / \mathrm{C}=0.111)$ 


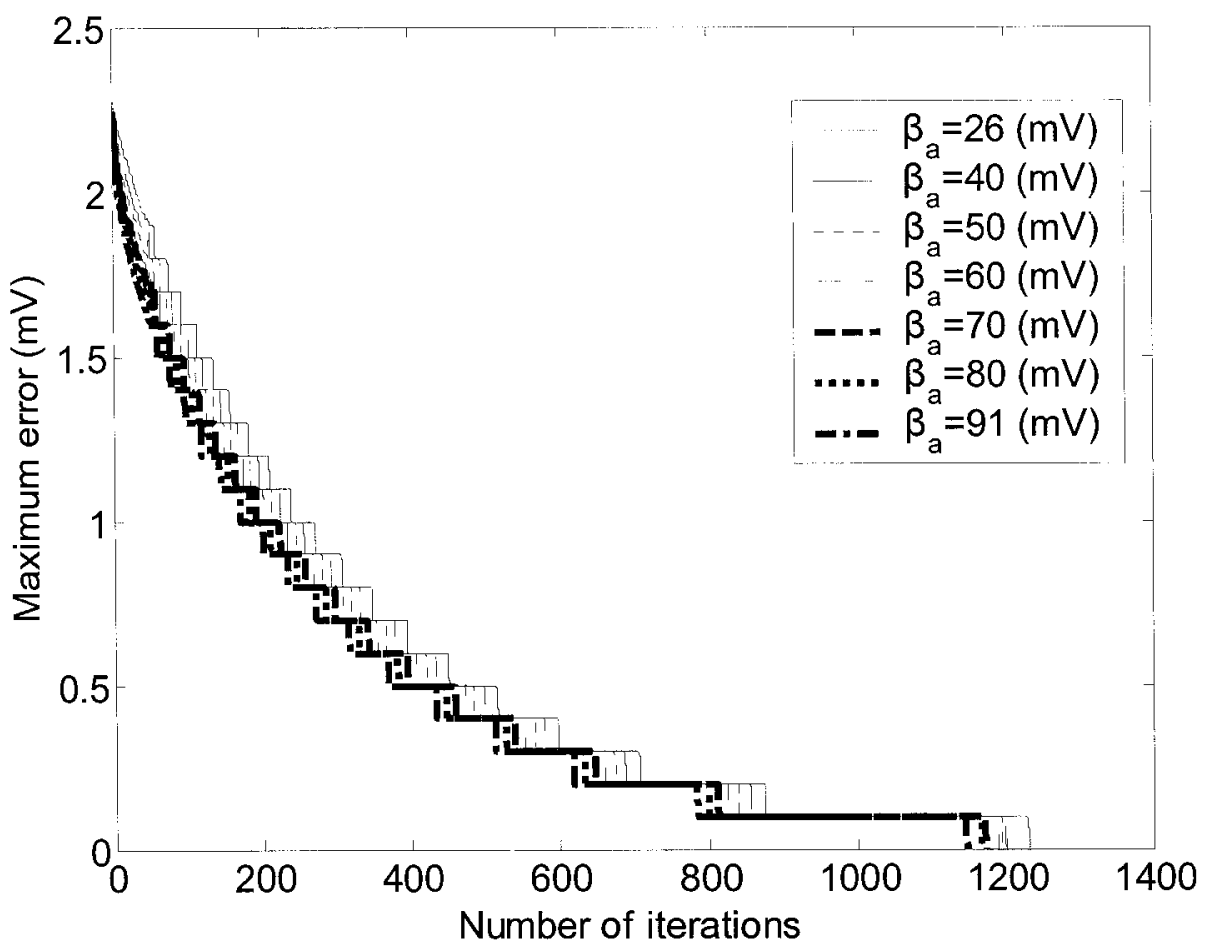

Fig. 6.3: The effect of anodic Tafel slope on convergence rate $(\mathrm{A} / \mathrm{C}=0.111)$

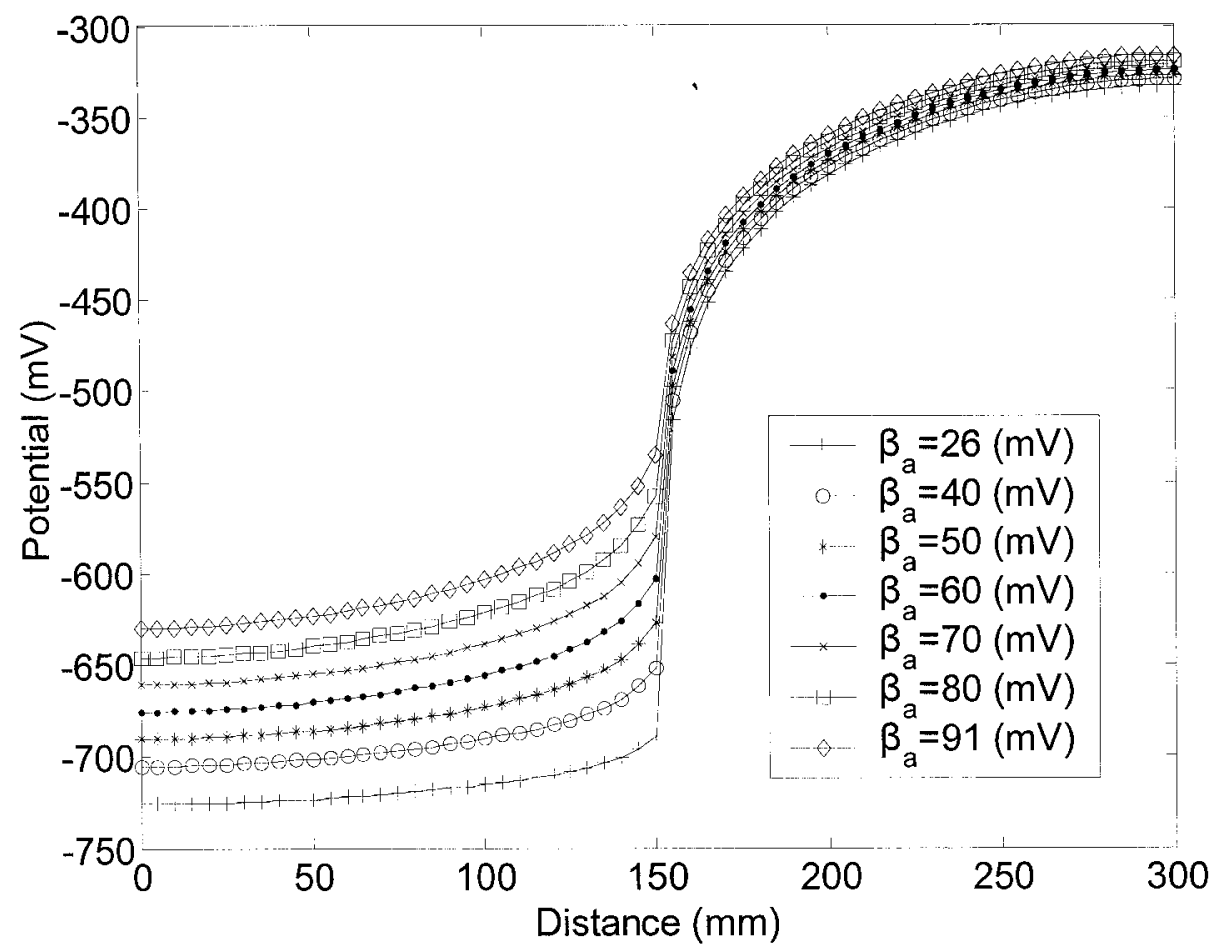

Fig. 6.4: The effect of anodic Tafel slope on potential distribution $(\mathrm{A} / \mathrm{C}=1.0)$ 


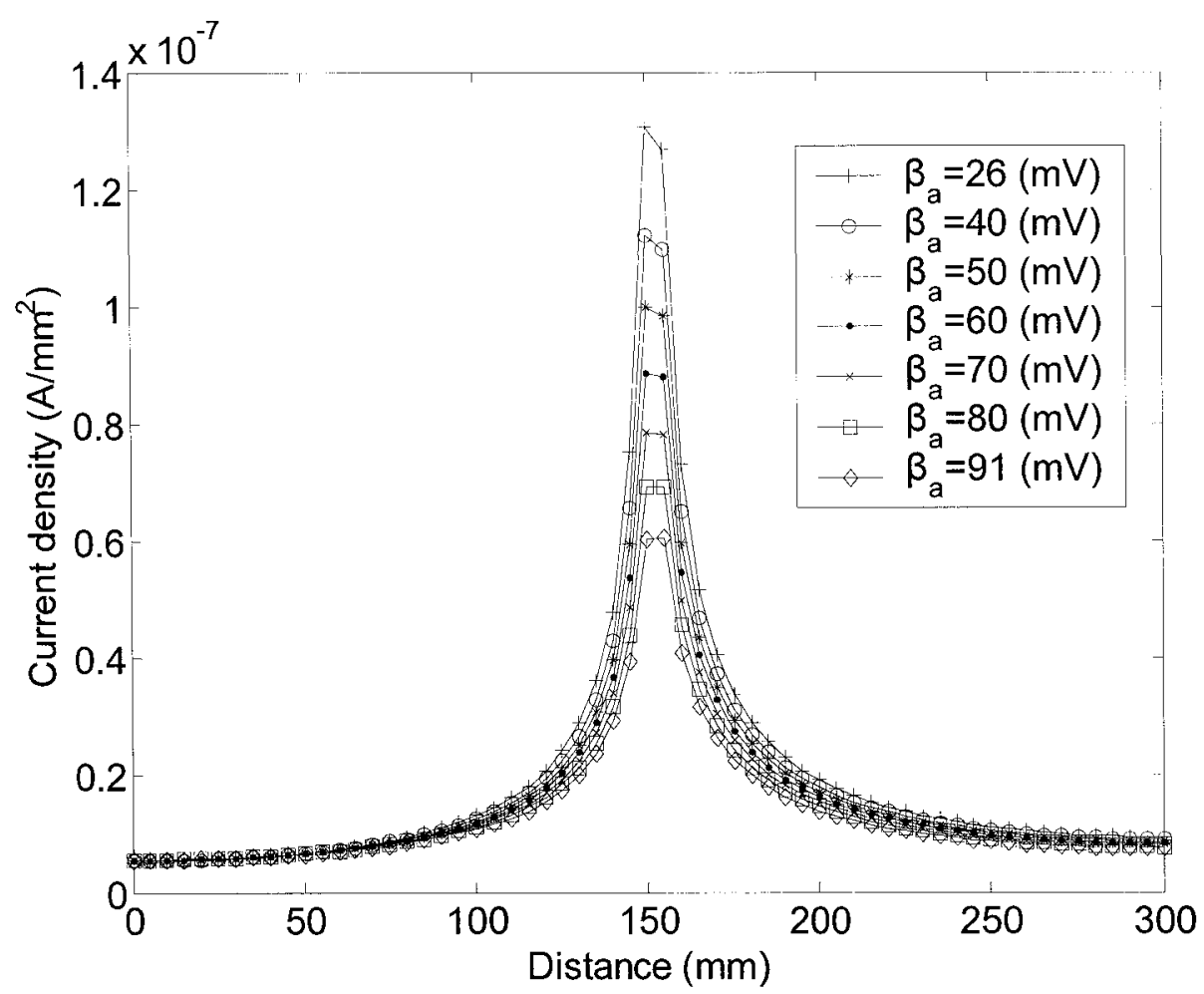

Fig. 6.5: The effect of anodic Tafel slope on current density $(\mathrm{A} / \mathrm{C}=1.0)$

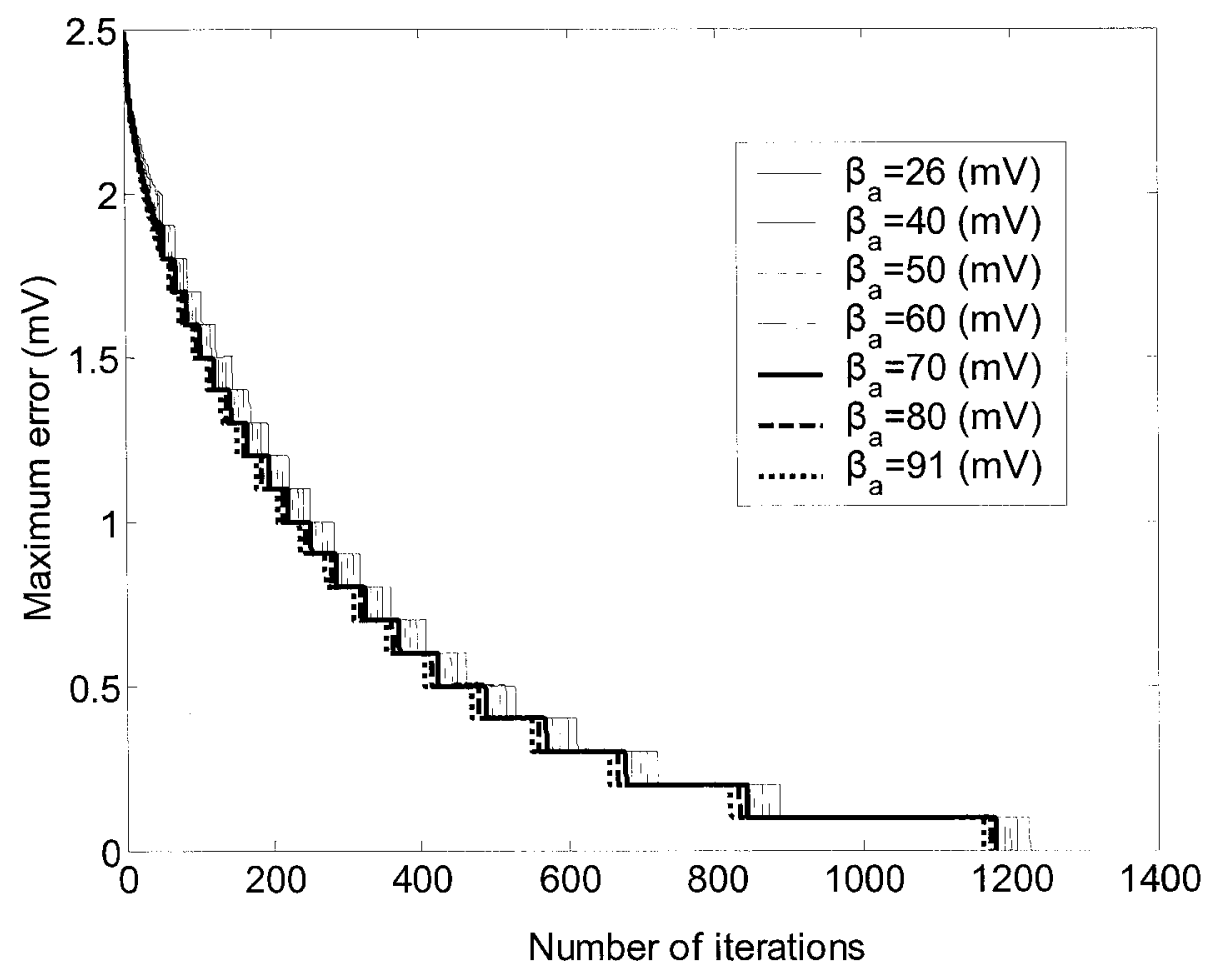

Fig. 6.6: The effect of anodic Tafel slope on convergence rate $(\mathrm{A} / \mathrm{C}=1.0)$ 


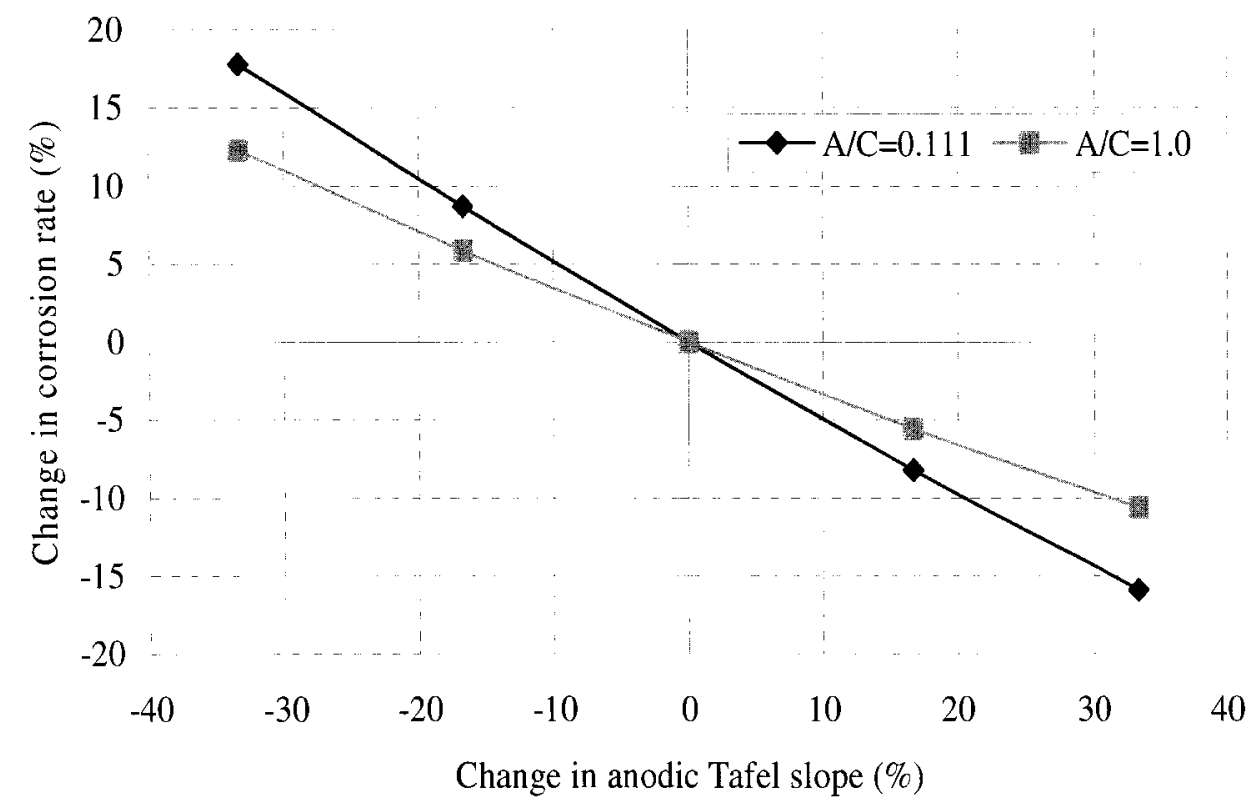

Fig. 6.7: The change in corrosion current rate vs. the change in anodic Tafel slope

\subsection{Investigation of the cathodic Tafel slope}

The effect of cathodic Tafel slope, $\beta_{c}$, on the corrosion rate of steel in concrete is studied by varying its value from $140 \mathrm{mV}$ to $230 \mathrm{mV}$. This range can be considered to be representative of the cathodic Tafel slope in corrosion of steel in concrete. One of the steps in this range is $\beta_{c}=160 \mathrm{mV}$, which corresponds to the base case. An unconventionally low value, $\beta_{c}=100 \mathrm{mV}$, was also analyzed for verification reasons. The results of the analysis for $\mathrm{A} / \mathrm{C}=0.111$ and $\mathrm{A} / \mathrm{C}=1.0$ are shown in Figs. 6.8-6.10 and Figs. $6.11-6.13$, respectively.

As illustrated in Figs. 6.8 and 6.9 , for $\mathrm{A} / \mathrm{C}=0.111$, the change in $\beta_{c}$ only affects potential and current density distribution along the cathodic region. It can be observed in Fig. 6.8 that, as $\beta_{c}$ increases, the potential on the cathodic steel surface decreases; 
however the potential distribution along the anodic is not affected significantly. Therefore, as $\beta_{c}$ increases, the potential difference between the anodic and cathodic regions decrease, and this results in lower corrosion rates, as it can be observed in Fig. 6.9. The change in $\beta_{c}$ did not have a significant influence on the convergence rate of the numerical solution, as shown in Fig. 6.10. As illustrated in Figs. 6.11-6.13, similar qualitative observations can be made for $\mathrm{A} / \mathrm{C}=1.0$.

The change in corrosion rate vs. the change in cathodic Tafel slope for both $\mathrm{A} / \mathrm{C}$ ratios is plotted in Fig. 6.14. The corrosion rate in this plot is also taken as the average current density in the anodic region. As it can be seen from this figure, the relationship between the two variations is linear with very similar slopes, signifying that the effect of cathodic Tafel slope on the corrosion rate is not a function of $\mathrm{A} / \mathrm{C}$ ratio. The absolute values of slopes for both $\mathrm{A} / \mathrm{C}$ ratios are greater than 1.0 , which means that the effect of a certain amount of change in cathodic Tafel slope will cause greater change in corrosion rate. For example, as it can be observed in Fig. 6.14, a $10 \%$ deviation in $\beta_{c}$ from the base case results in more than $20 \%$ change in corrosion rate. 


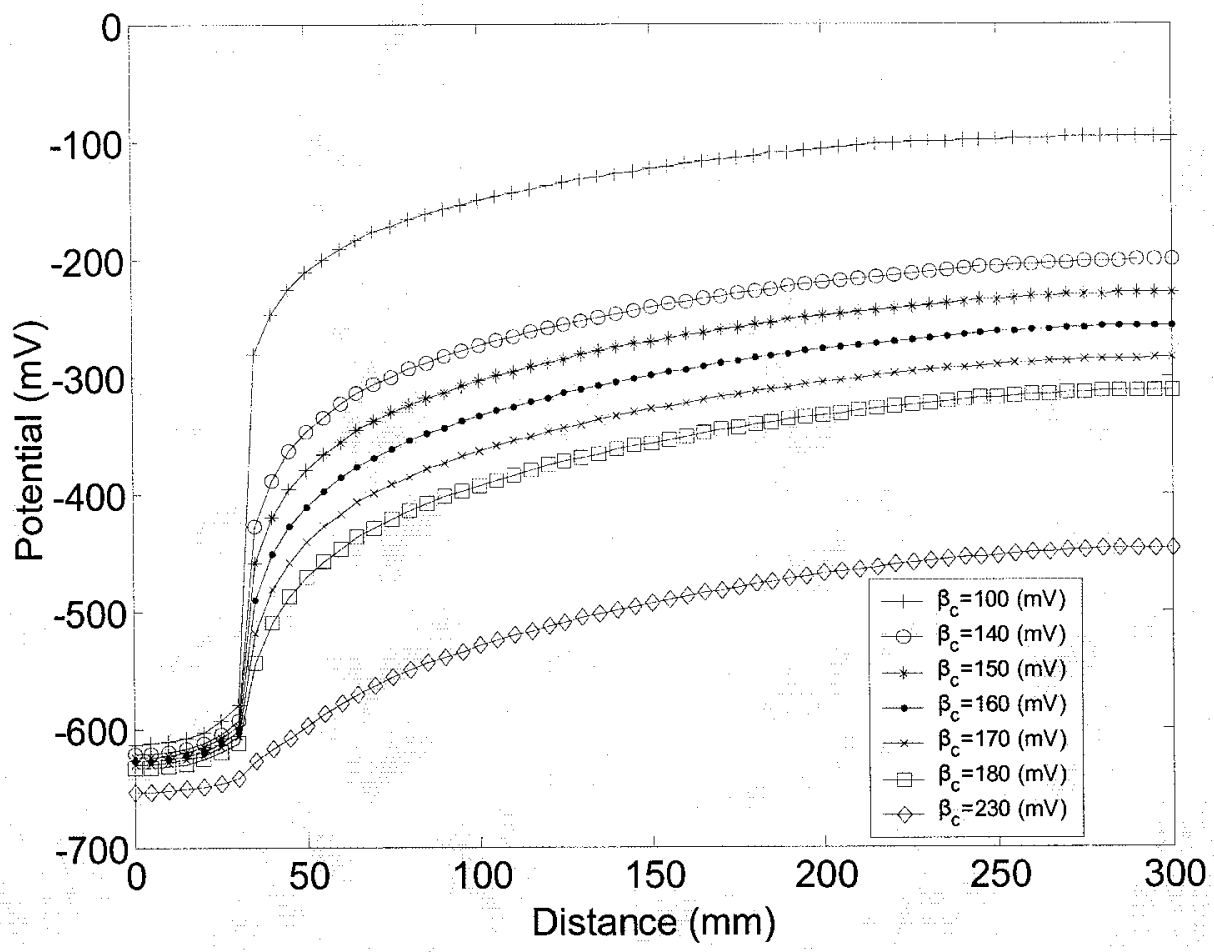

Fig. 6.8: The effect of cathodic Tafel slope on potential distribution $(\mathrm{A} / \mathrm{C}=$ $0.111)$

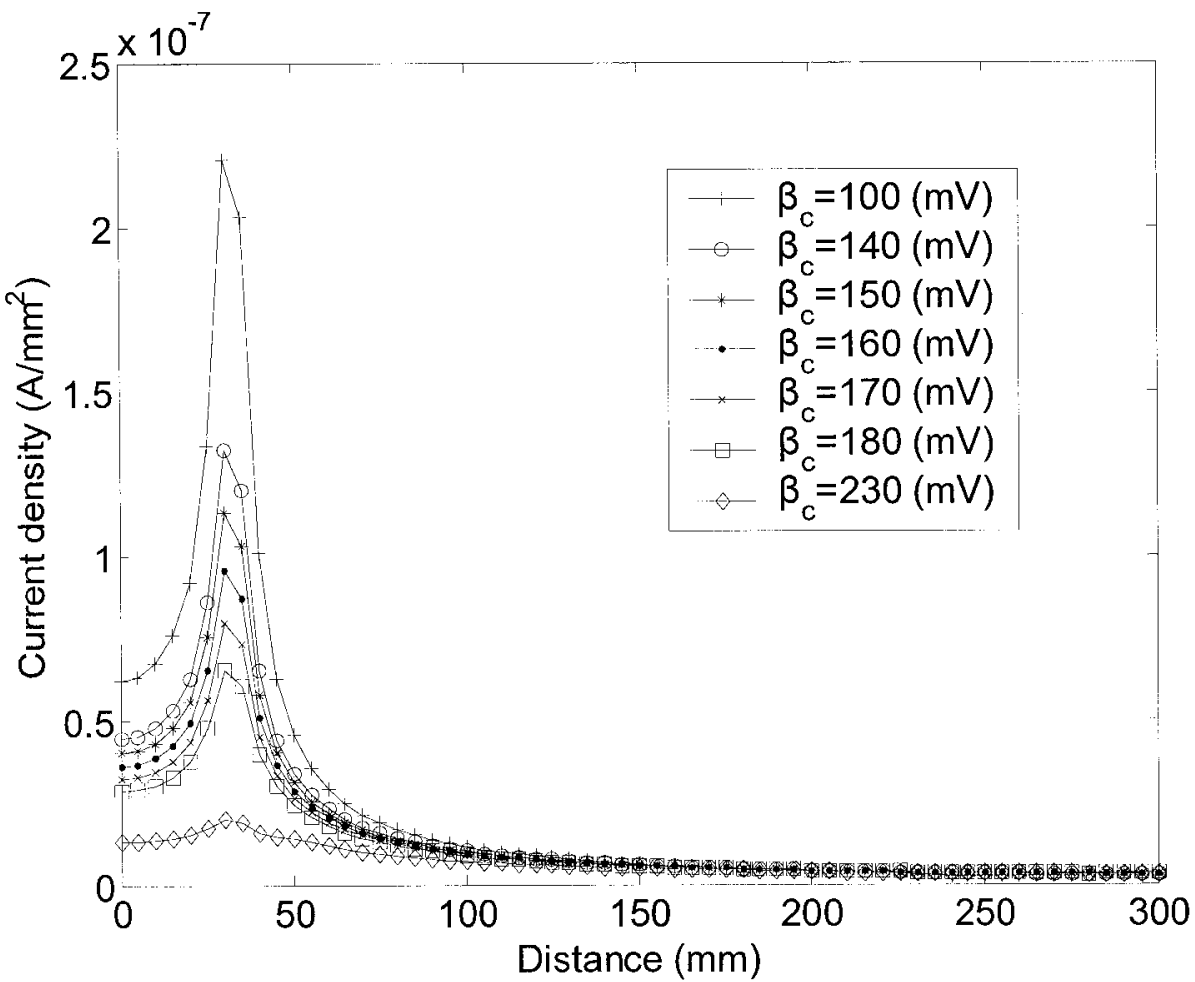

Fig. 6.9: The effect of cathodic Tafel slope on current density $(\mathrm{A} / \mathrm{C}=0.111)$ 


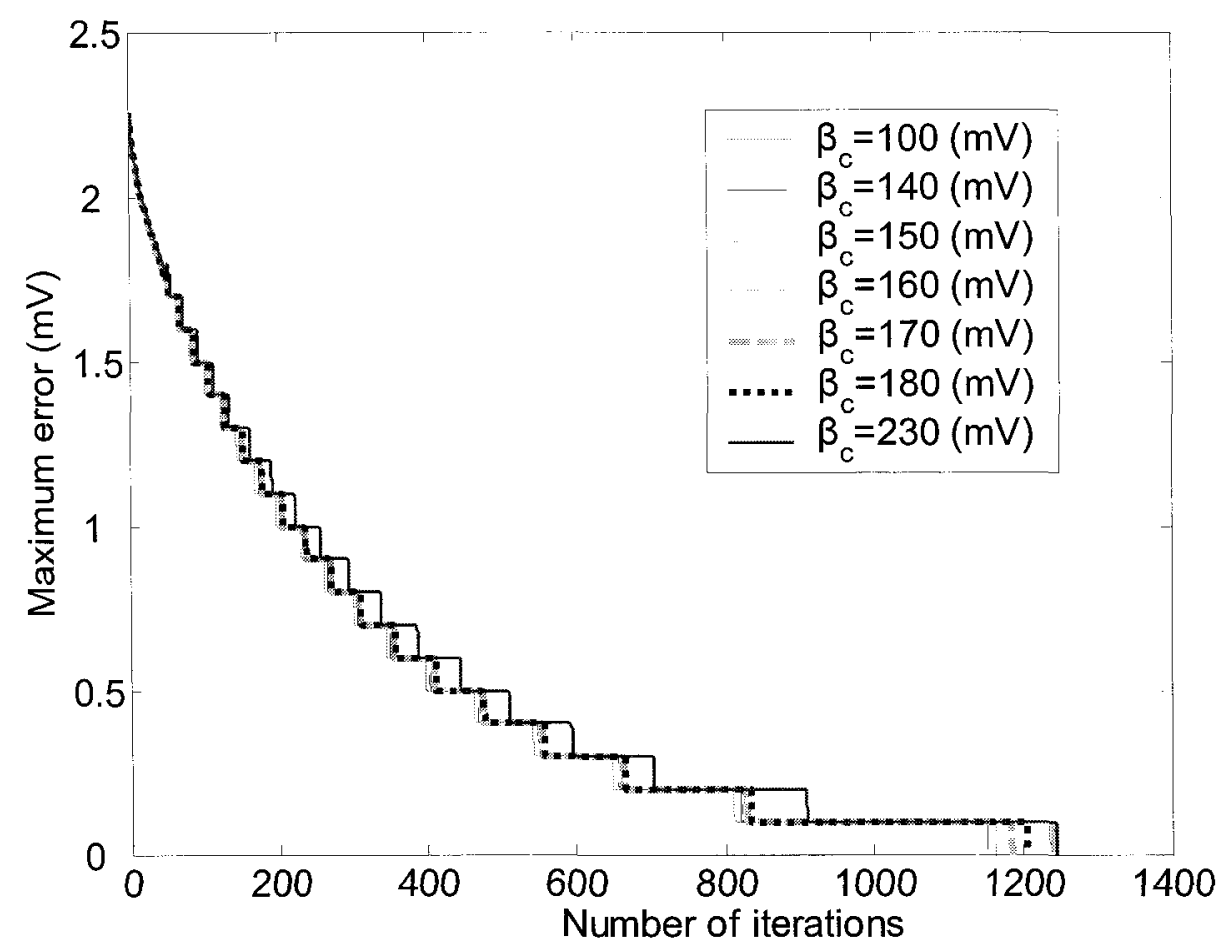

Fig. 6.10: The effect of anodic Tafel slope on convergence rate $(\mathrm{A} / \mathrm{C}=0.111)$

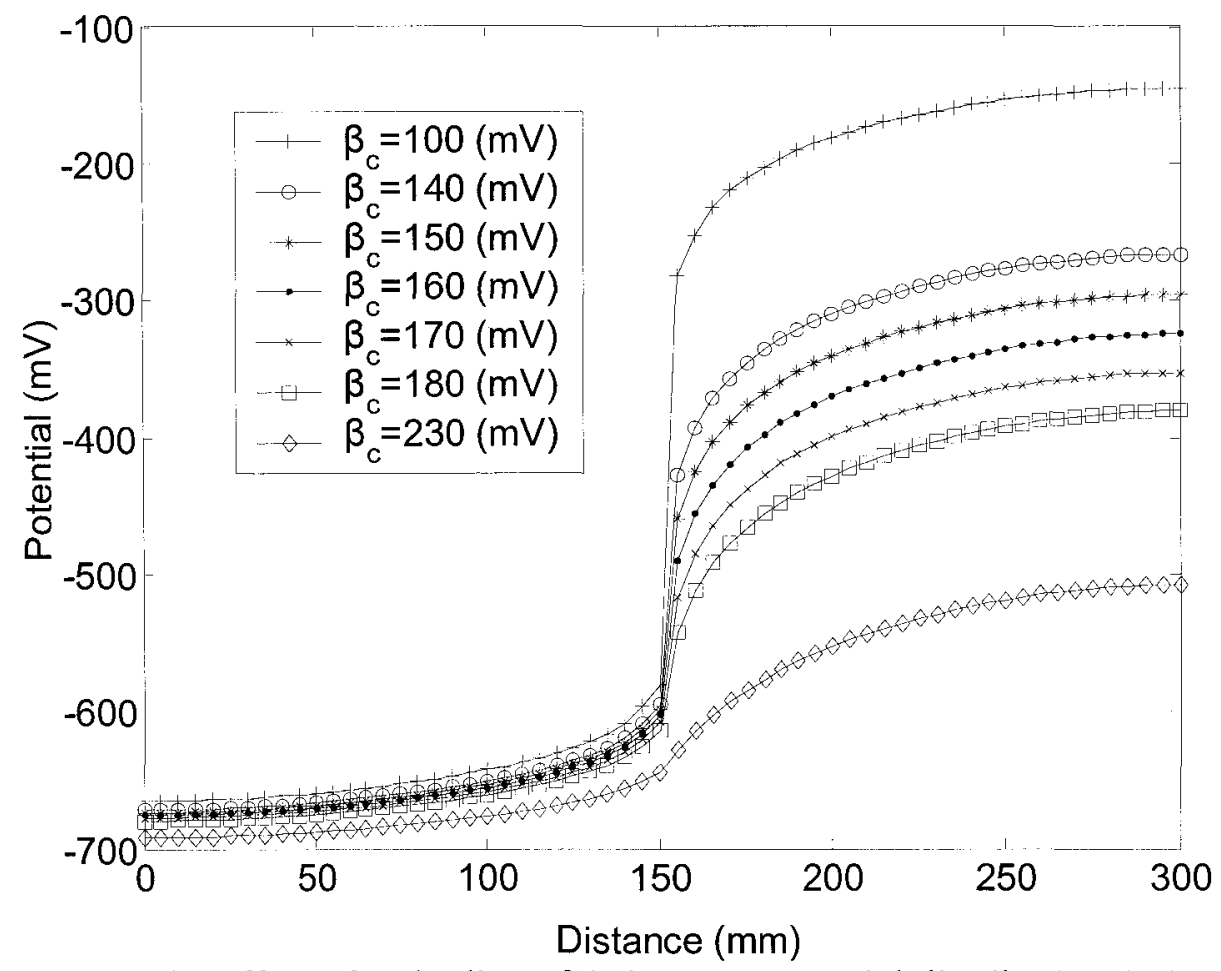

Fig. 6.11: The effect of cathodic Tafel slope on potential distribution $(\mathrm{A} / \mathrm{C}=1.0)$ 


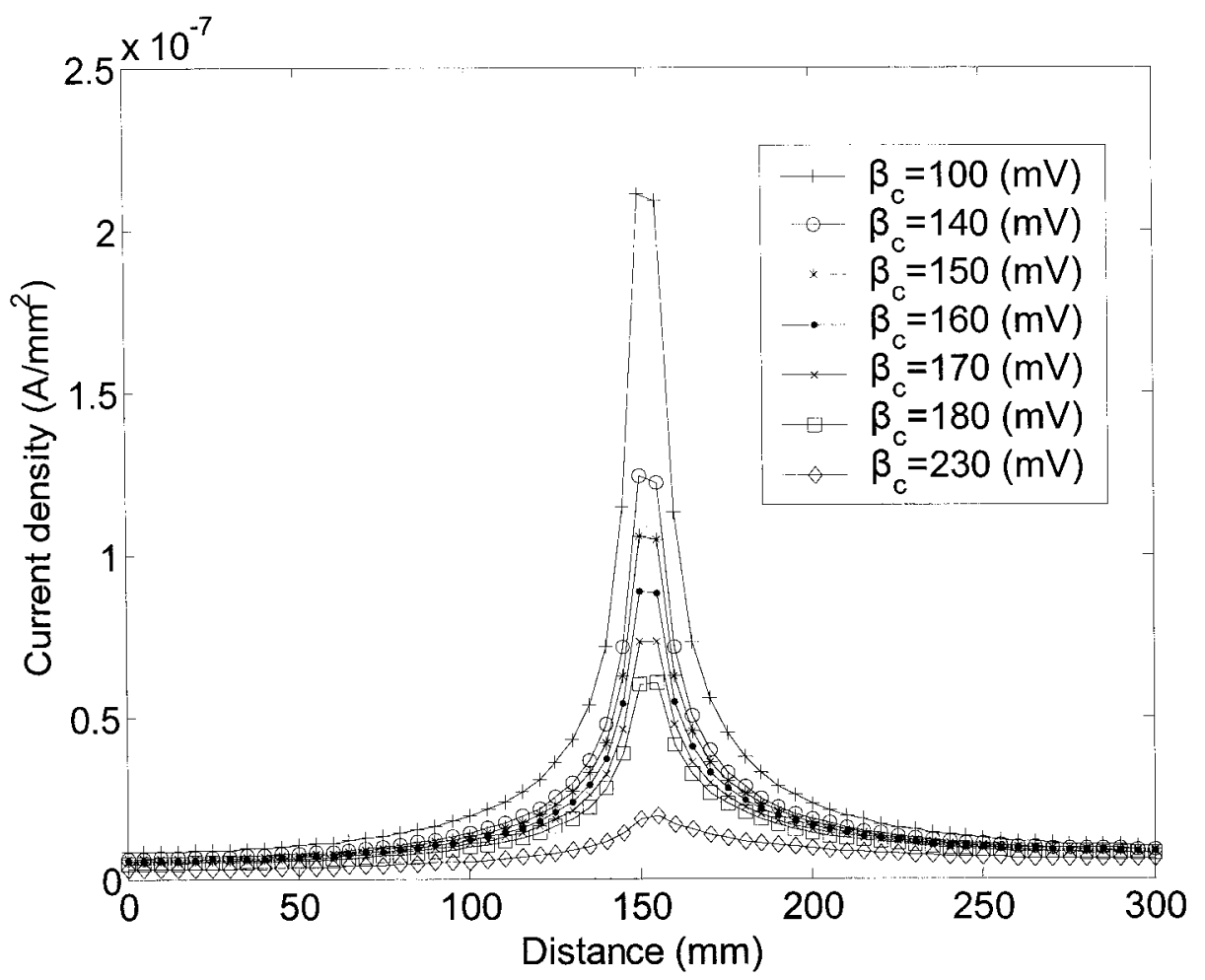

Fig. 6.12: The effect of cathodic Tafel slope on current density $(\mathrm{A} / \mathrm{C}=1.0)$

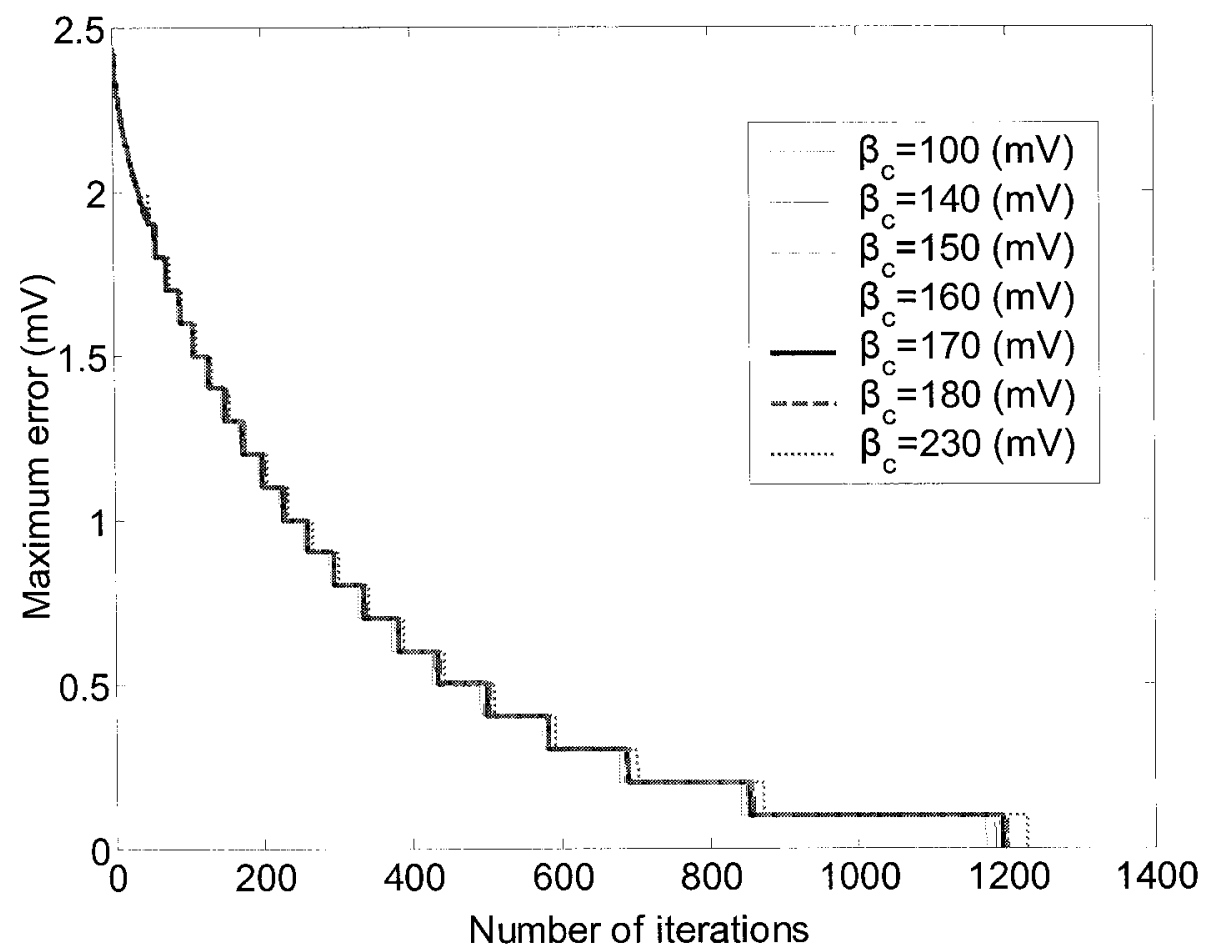

Fig. 6.13: The effect of anodic Tafel slope on convergence rate $(\mathrm{A} / \mathrm{C}=1.0)$ 


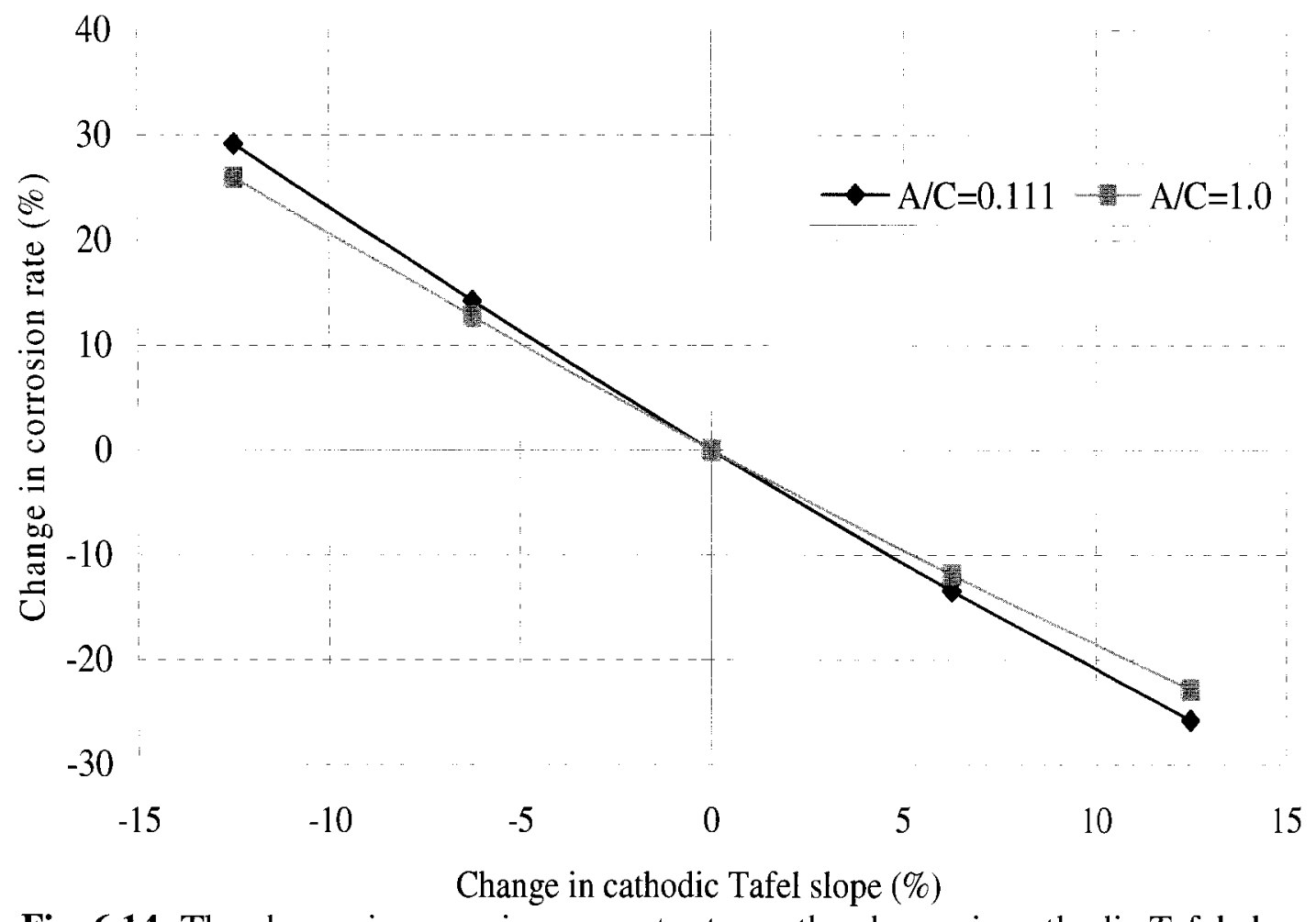

\subsection{Investigation of the anodic exchange current density}

The effect of the anodic exchange current density, $i_{o a}$, on the corrosion rate was studied by varying its value from $1 \times 10^{-12} \mathrm{~A} / \mathrm{mm}^{2}$ to $374 \times 10^{-12} \mathrm{~A} / \mathrm{mm}^{2}$. This range is a representation of the boundaries of the values used in the literature. One of the steps in this range, $i_{o a}=187.5 \times 10^{-12} \mathrm{~A} / \mathrm{mm}^{2}$, corresponds to the base case. The results of the analysis for $\mathrm{A} / \mathrm{C}=0.111$ and $\mathrm{A} / \mathrm{C}=1.0$ are shown in Figs. 6.15-6.17 and Figs. 6.18-6.20, respectively.

As illustrated in Figs. 6.15 and 6.16 , for $\mathrm{A} / \mathrm{C}=0.111$, the change in $i_{r a}$ primarily affects potential and current density distribution along the anodic region. It can be observed in 
Fig. 6.15 that, as $i_{o a}$ increases, the potential on the anodic steel surface decreases; however the potential distribution along the cathodic surface is not affected significantly. Therefore, as $i_{o a}$ increases, the potential difference between the anodic and cathodic regions increase, and this results in higher corrosion rates, as it can be observed in Fig. 6.16. As in previous cases, the change in $i_{o d}$ did not have a significant influence on the convergence rate of the numerical solution, as shown in Fig. 6.17. As illustrated in Figs. 6.18-6.20, similar qualitative observations can be made for $\mathrm{A} / \mathrm{C}=1.0$.

The change in corrosion rate vs. the change in $i_{o a}$ for both $\mathrm{A} / \mathrm{C}$ ratios is plotted in Fig. 6.21. The corrosion rate in this plot is also taken as the average current density in the anodic region. As it can be seen from this figure, the relationship between the two variations is non-linear with very similar curves, signifying that the effect of $i_{o a}$ on the corrosion rate is not a function of $\mathrm{A} / \mathrm{C}$ ratio. For positive variations from the base case, however, the relationship is linear. For a range between $-50 \%$ and $+50 \%$ variation from the base case, the relationship can also be considered linear. For negative variations beyond $-50 \%$ from the base case, the corrosion rate changes in an increasing rate. It should be noted that the slope of the linear portion of the curves is rather small, i.e. less than 0.1 , signifying that the variation of $i_{o a}$ is not a significant factor in determining the corrosion rate, as long as the quantity is selected within a realistic range. 


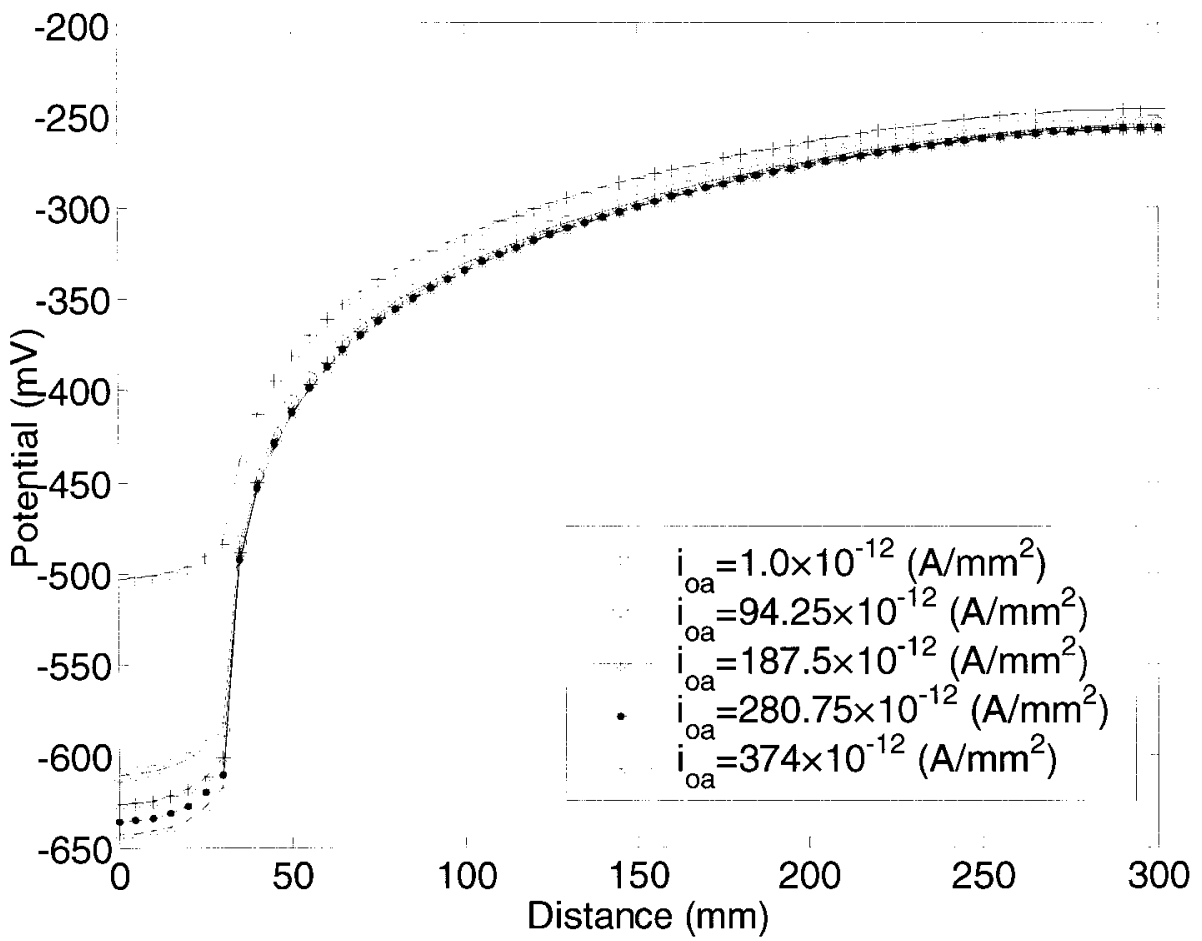

Fig. 6.15: The effect of $i_{o a}$ on potential distribution $(\mathrm{A} / \mathrm{C}=0.111)$

$1.4^{\times 10^{-7}}$

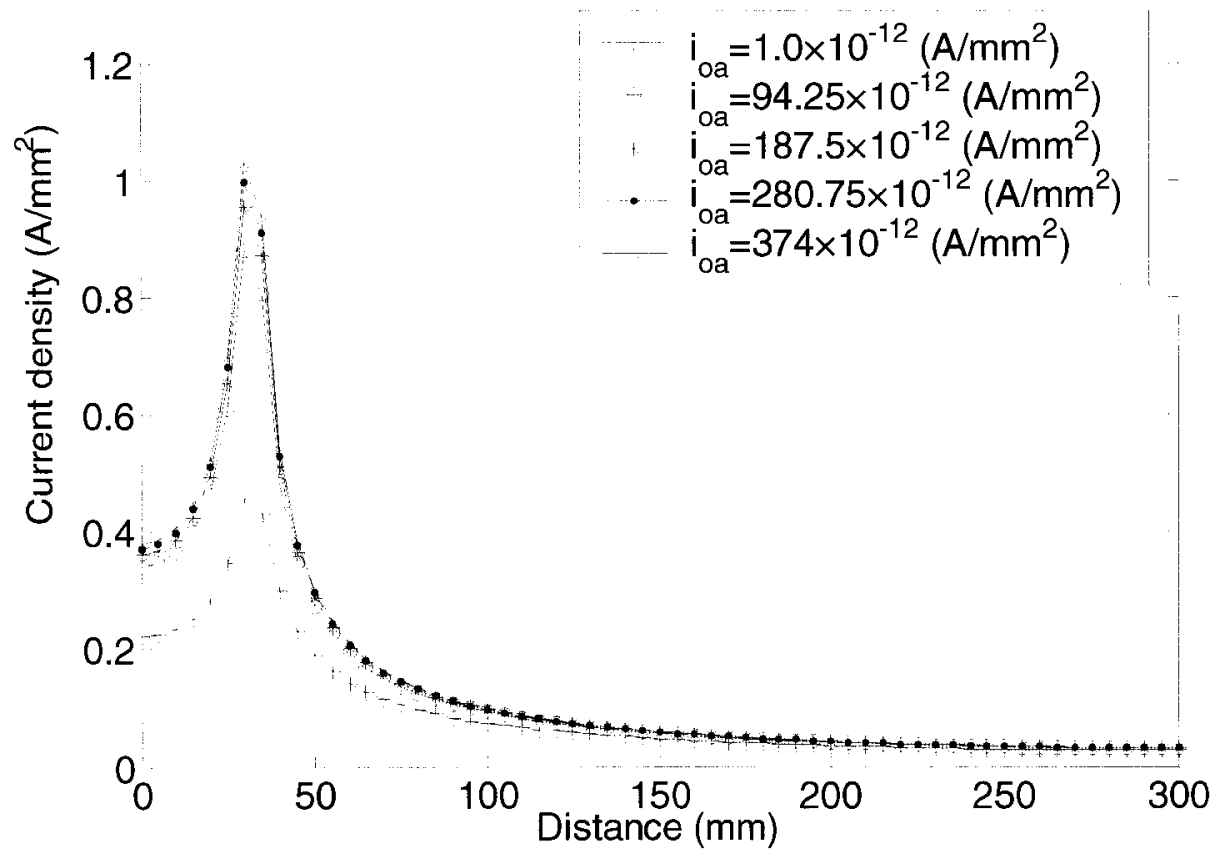

Fig. 6.16: The effect of $i_{o c}$ on current density $(\mathrm{A} / \mathrm{C}=0.111)$ 


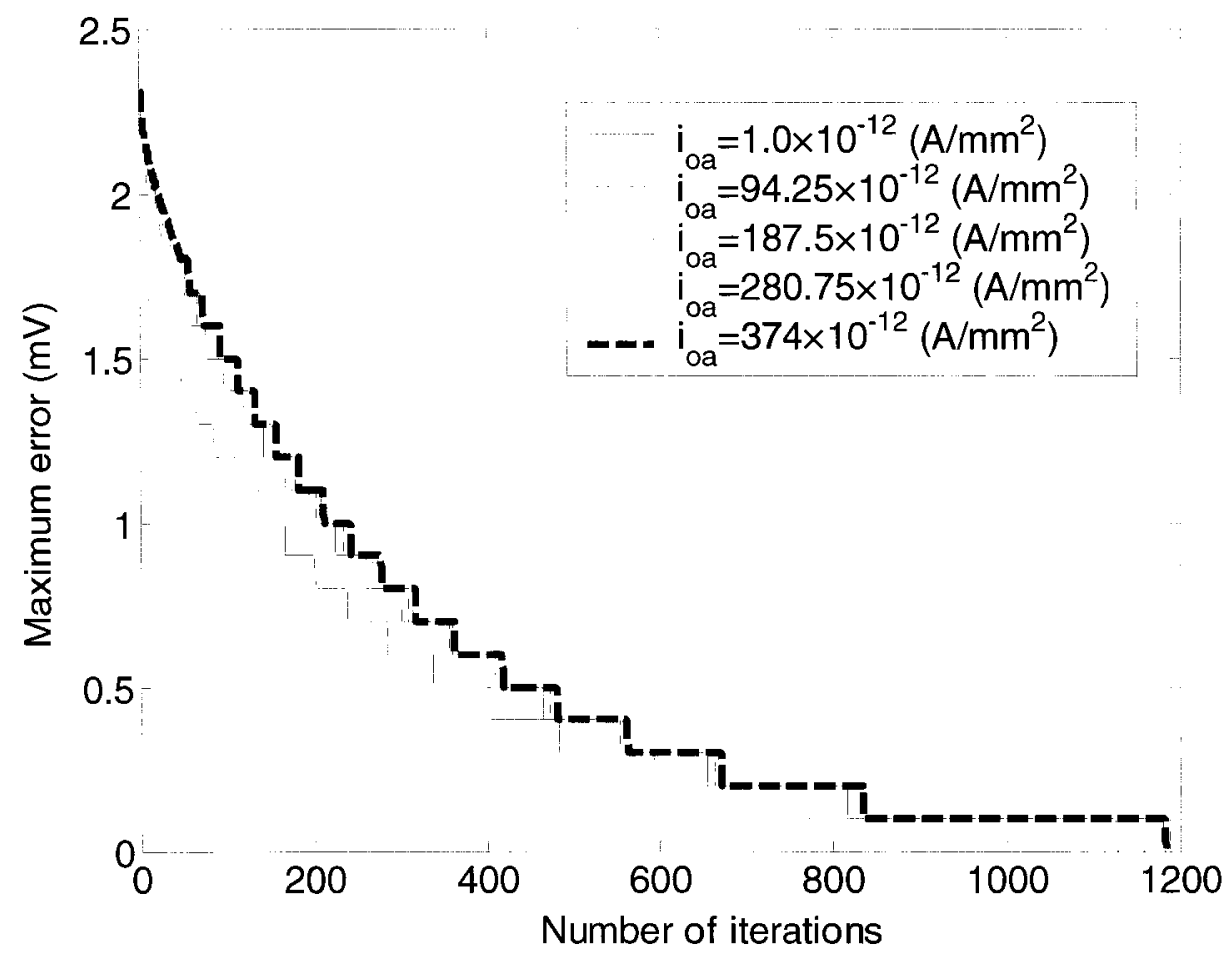

Fig. 6.17: The effect of $i_{o a}$ on convergence rate $(\mathrm{A} / \mathrm{C}=0.111)$

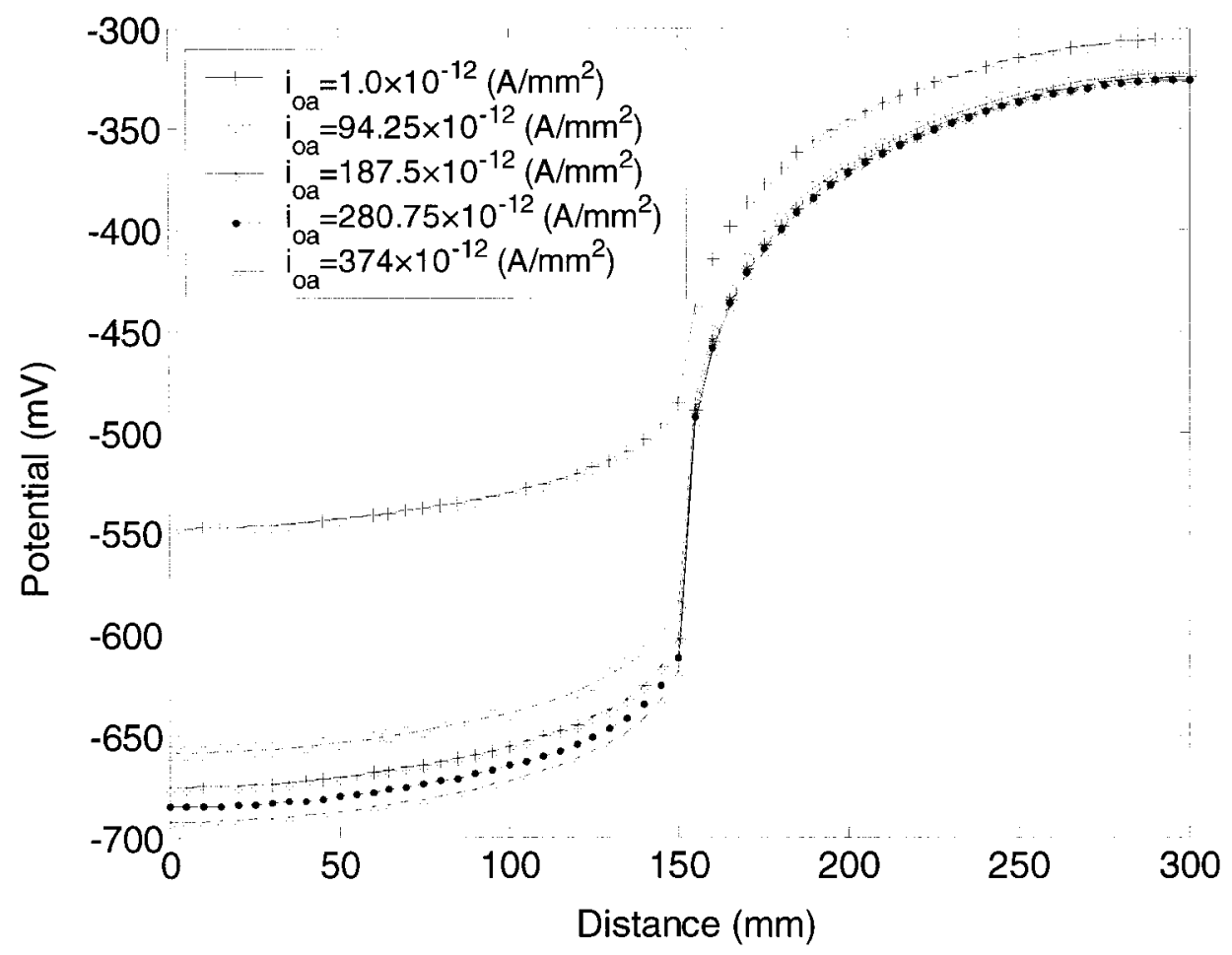

Fig. 6.18: The effect of $i_{o \iota}$ on potential distribution $(\mathrm{A} / \mathrm{C}=1.0)$ 


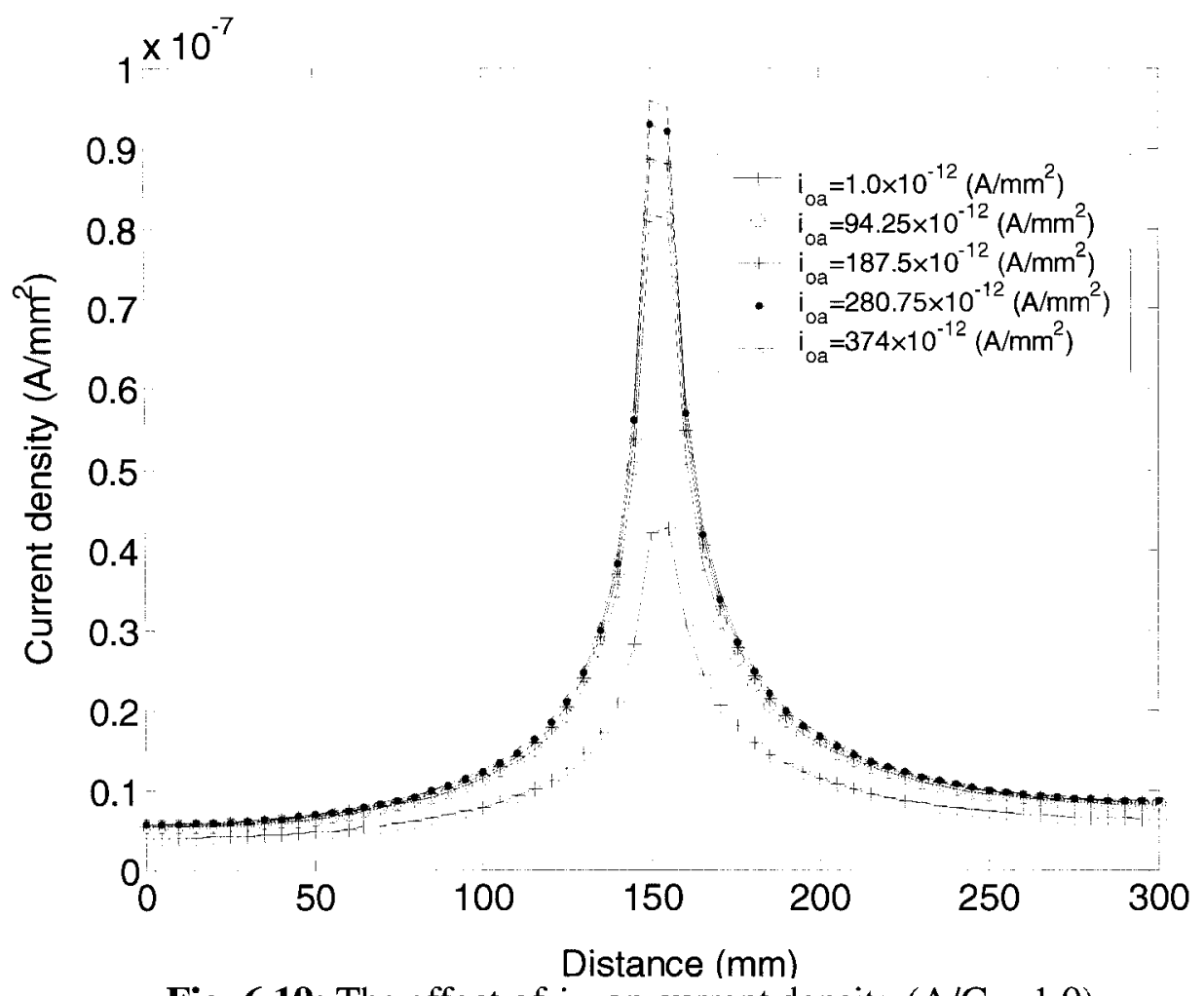

Fig. 6.19: The effect of $i_{o a}$ on current density $(\mathrm{A} / \mathrm{C}=1.0)$

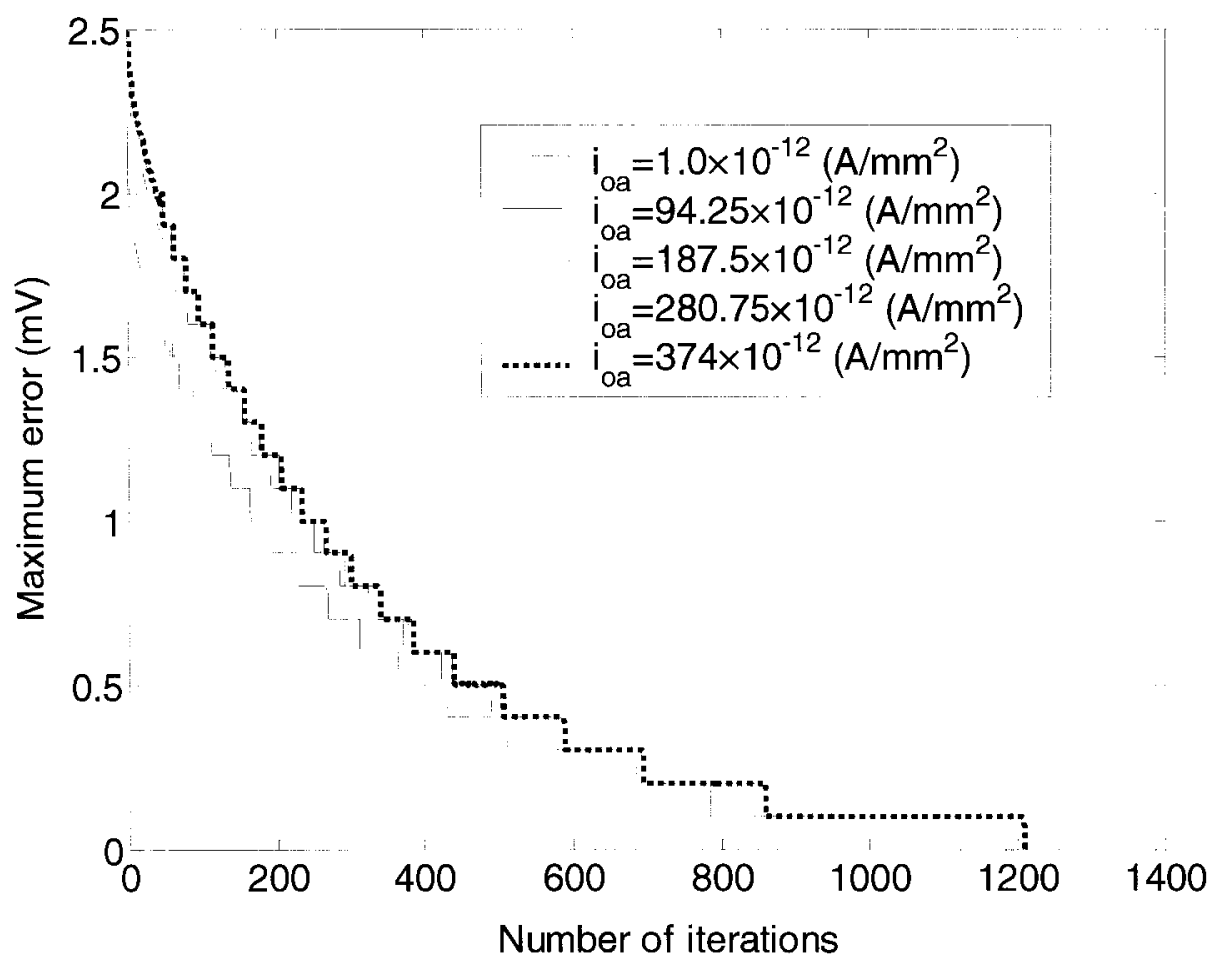

Fig. 6.20: The effect of $i_{o a}$ on convergence rate $(\mathrm{A} / \mathrm{C}=1.0)$ 


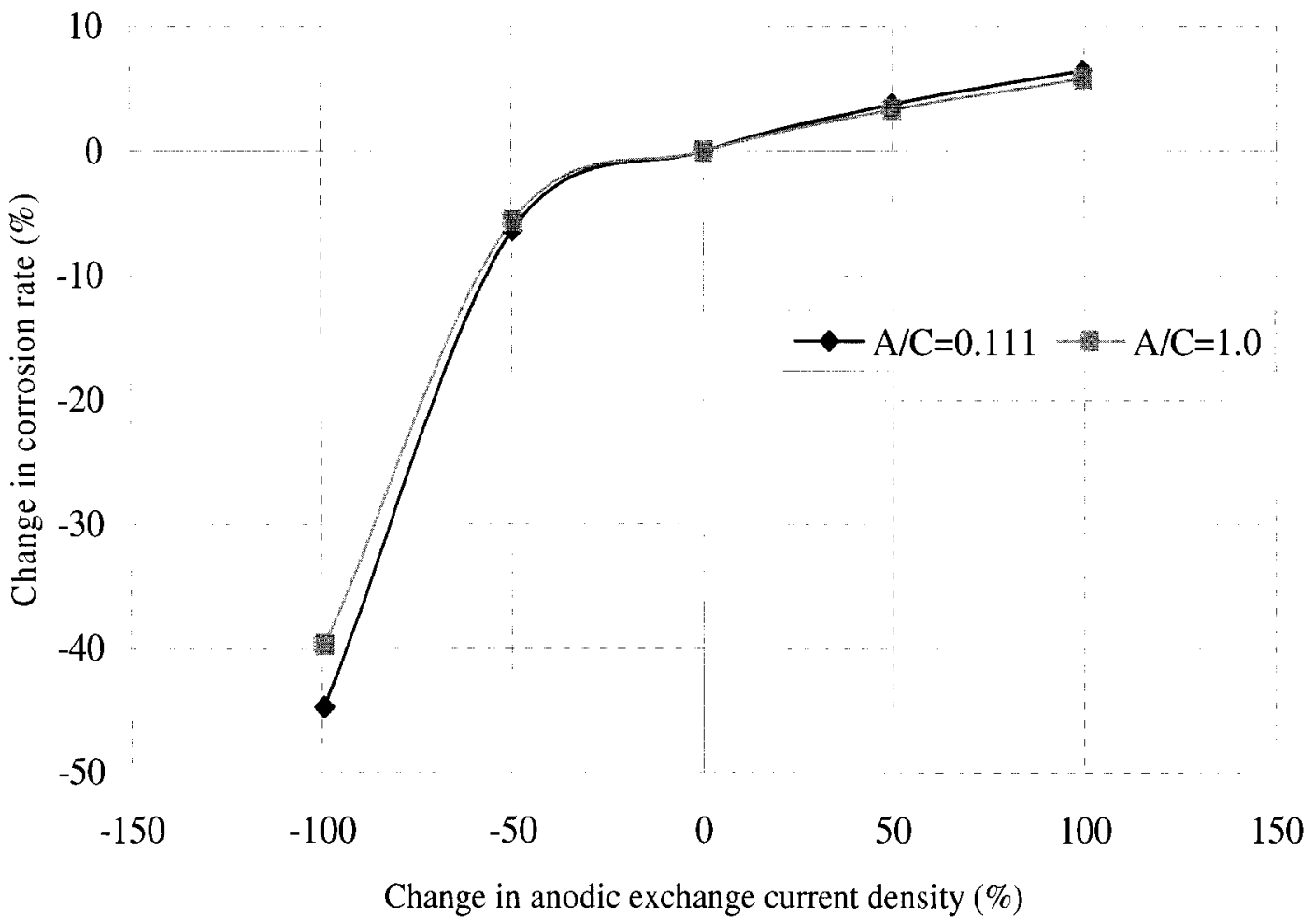

Fig. 6.21: The change in corrosion current rate vs. the anodic exchange current density

\subsection{Investigation of the cathodic exchange current density}

The effect of the cathodic exchange current density, $i_{o c}$, on the corrosion rate was studied by varying its value from $0.1 \times 10^{-12} \mathrm{~A} / \mathrm{mm}^{2}$ to $12.4 \times 10^{-12} \mathrm{~A} / \mathrm{mm}^{2}$. This range is a representation of the boundaries of the values used in the literature. One of the steps in this range, $i_{o c}=6.25 \times 10^{-12} \mathrm{~A} / \mathrm{mm}^{2}$, corresponds to the base case. The results of the analysis for $\mathrm{A} / \mathrm{C}=0.111$ and $\mathrm{A} / \mathrm{C}=1.0$ are shown in Figs. 6.22-6.24 and Figs. 6.25-6.27, respectively.

As illustrated in Figs. 6.22 and 6.23 , for $\mathrm{A} / \mathrm{C}=0.111$, the change in $i_{o c}$ primarily affects 
potential distribution along the cathodic region. It can be observed in Fig. 6.22 that, as $i_{o c}$ increases, the potential on the cathodic steel surface also increases; however the potential distribution along the anodic surface is not affected significantly. Therefore, as $i_{o c}$ increases, the potential difference between the anodic and cathodic regions increase, and this results in higher corrosion rates, as it can be observed in Fig. 6.23. As in previous cases, the change in $i_{o c}$ did not have a significant influence on the convergence rate of the numerical solution, as shown in Fig. 6.24. As illustrated in Figs. 6.25-6.27, similar qualitative observations can be made for $\mathrm{A} / \mathrm{C}=1.0$.

The change in corrosion rate vs. the change in $i_{o c}$ for both $\mathrm{A} / \mathrm{C}$ ratios is plotted in Fig. 6.28. The corrosion rate in this plot is also taken as the average current density in the anodic region. As it can be seen from this figure, the relationship between the two variations is non-linear with very similar curves, signifying that the effect of $i_{o c}$ on the corrosion rate is not a function of $\mathrm{A} / \mathrm{C}$ ratio. For positive variations from the base case, however, the relationship is linear. For a range between $-50 \%$ and $+100 \%$ variation from the base case, the relationship can also be considered linear. For negative variations beyond $-50 \%$ from the base case, the corrosion rate changes in an increasing rate. Actually, in this range, the effect of $i_{o c}$ on the corrosion rate can be significant. 


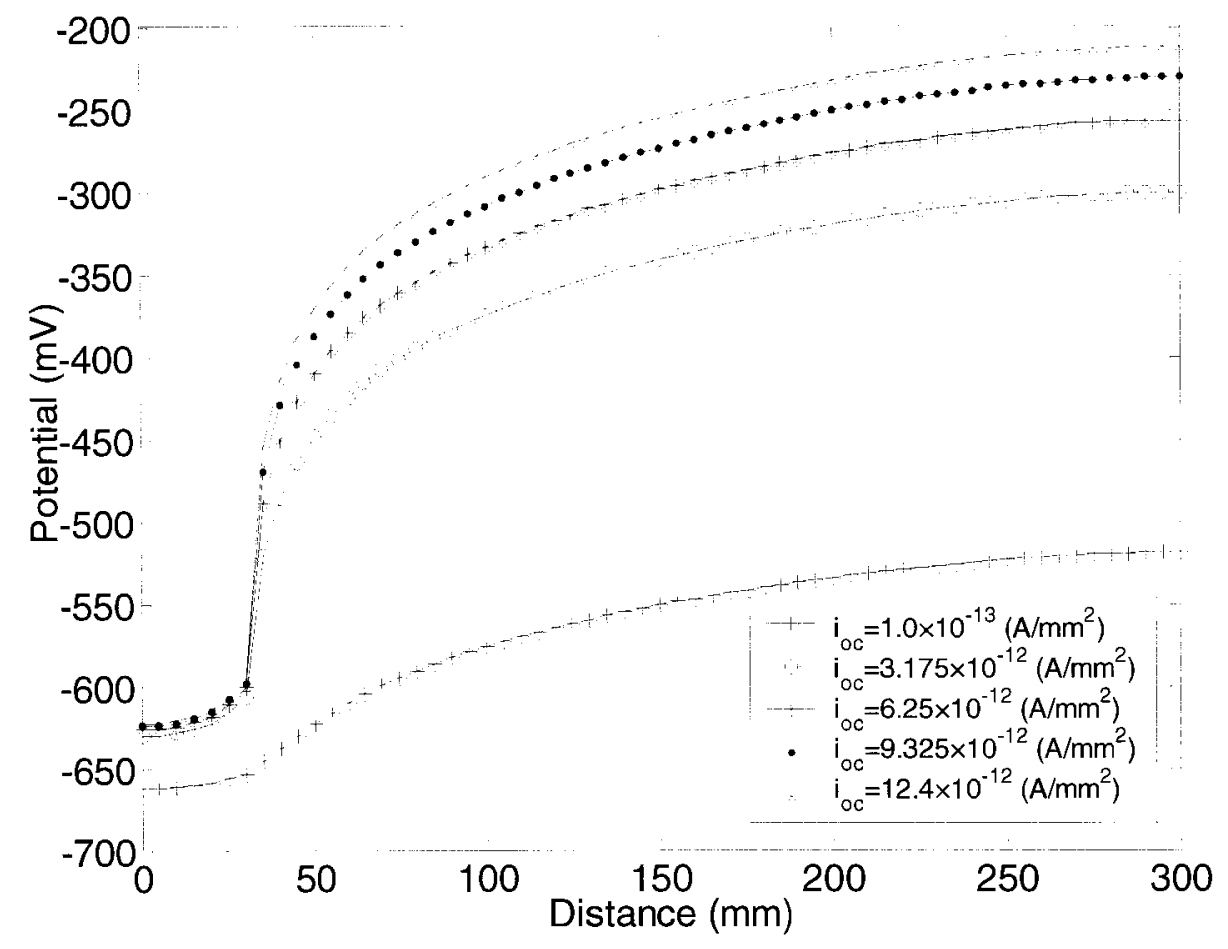

Fig. 6.22: The effect of $i_{o c}$ on potential distribution $(\mathrm{A} / \mathrm{C}=0.111)$

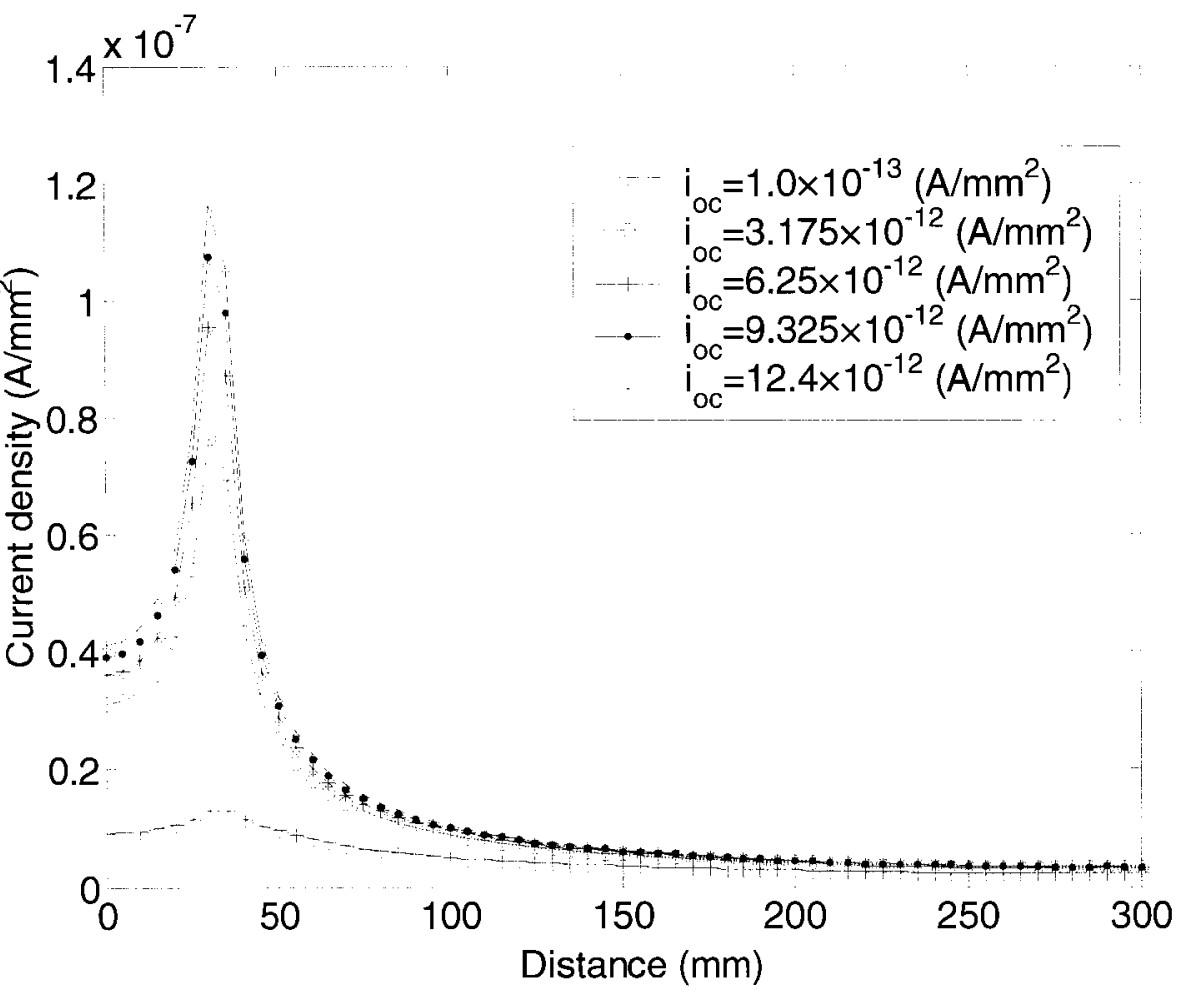

Fig. 6.23: The effect of $i_{o c}$ on current density $(\mathrm{A} / \mathrm{C}=0.111)$ 


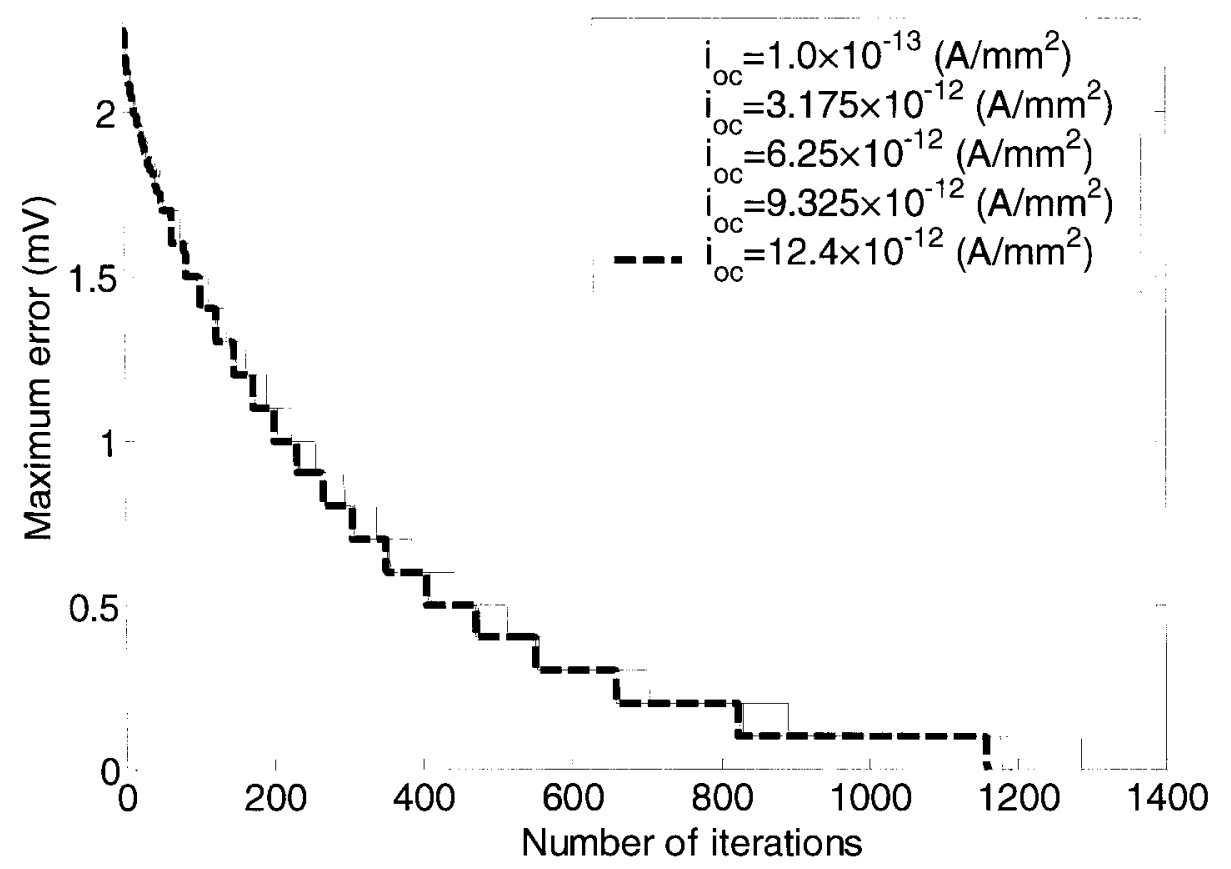

Fig. 6.24: The effect of $i_{o c}$ on convergence rate $(\mathrm{A} / \mathrm{C}=0.111)$

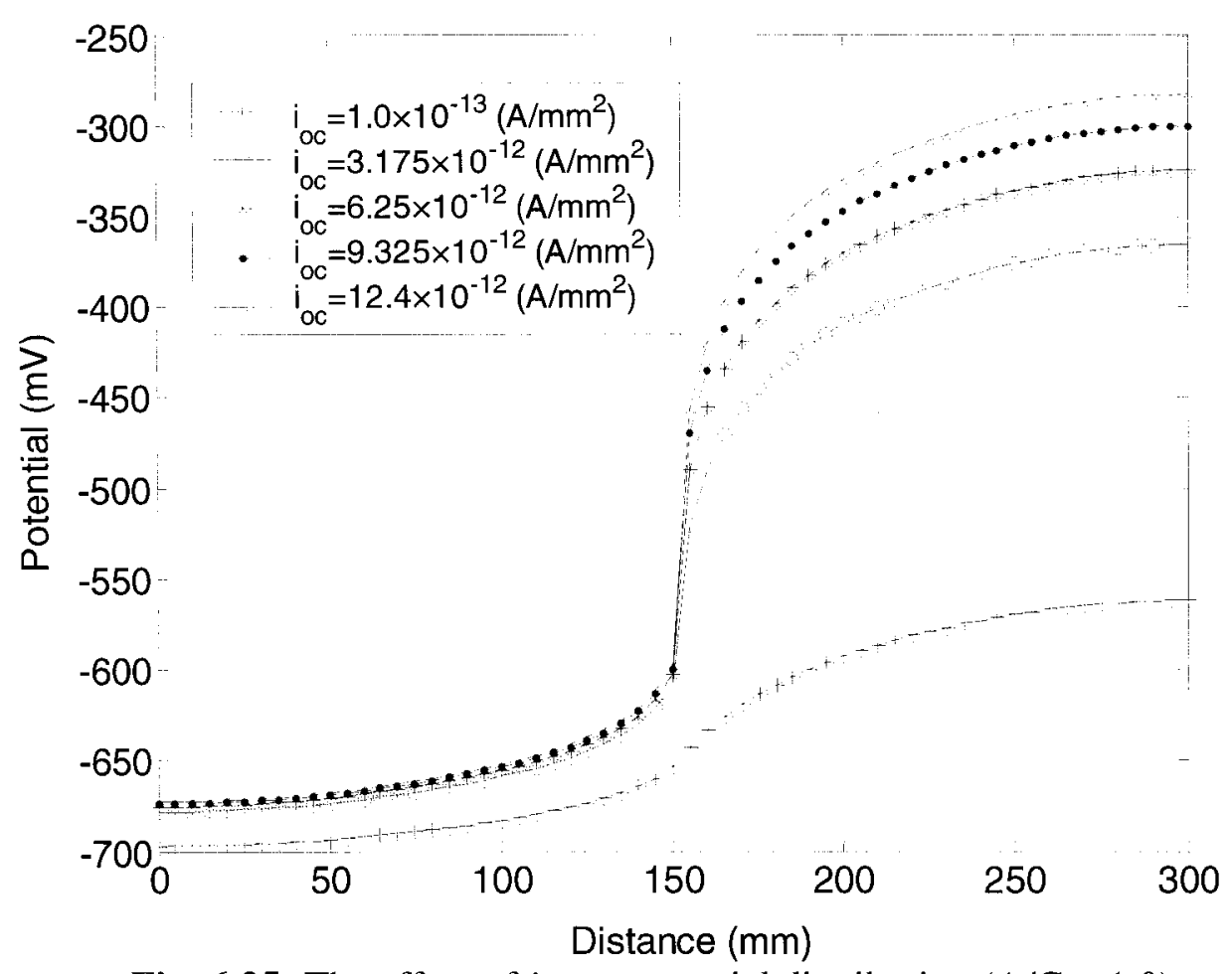

Fig. 6.25: The effect of $i_{o c}$ on potential distribution $(\mathrm{A} / \mathrm{C}=1.0)$ 


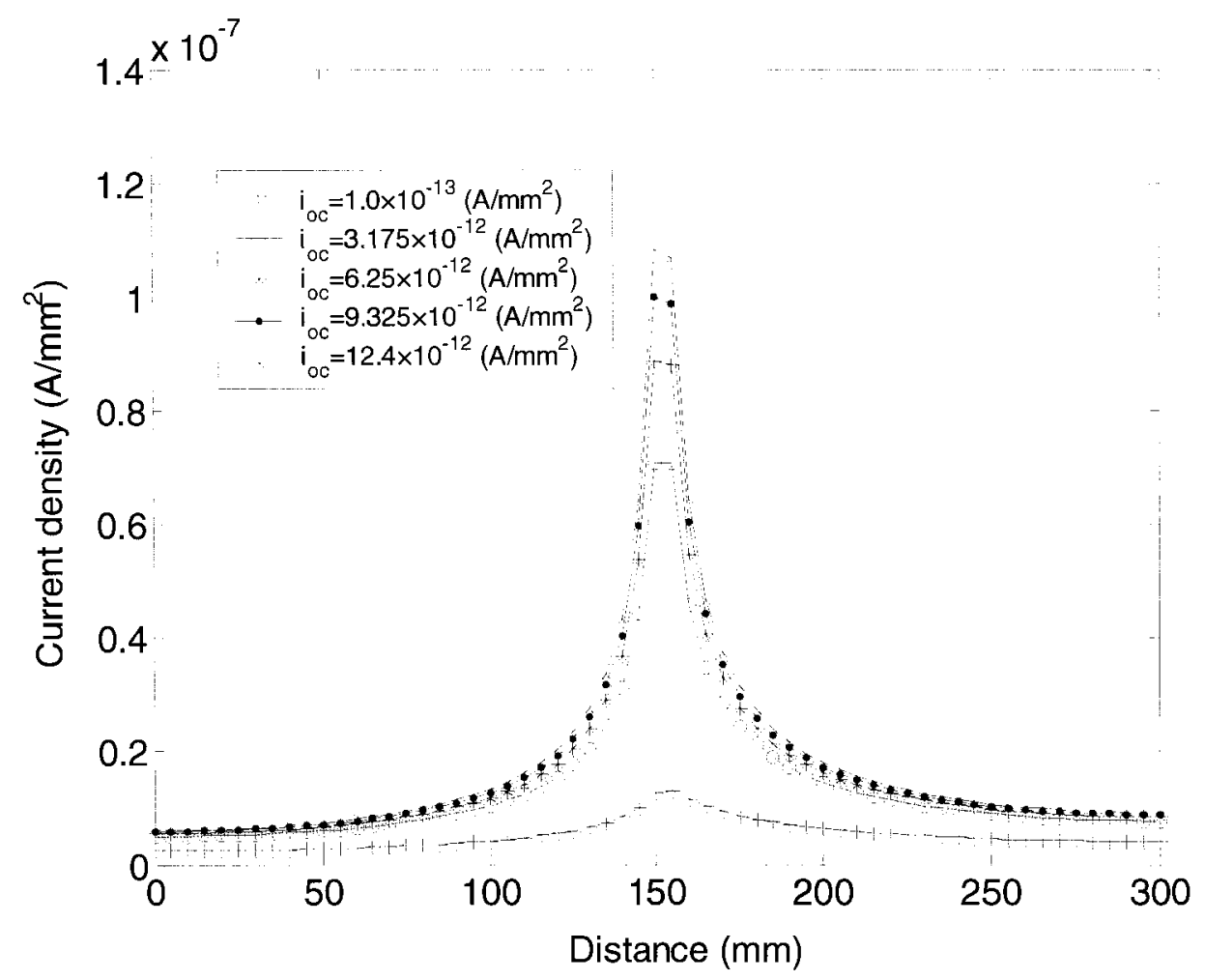

Fig. 6.26: The effect of $i_{o c}$ on current density $(\mathrm{A} / \mathrm{C}=1.0)$

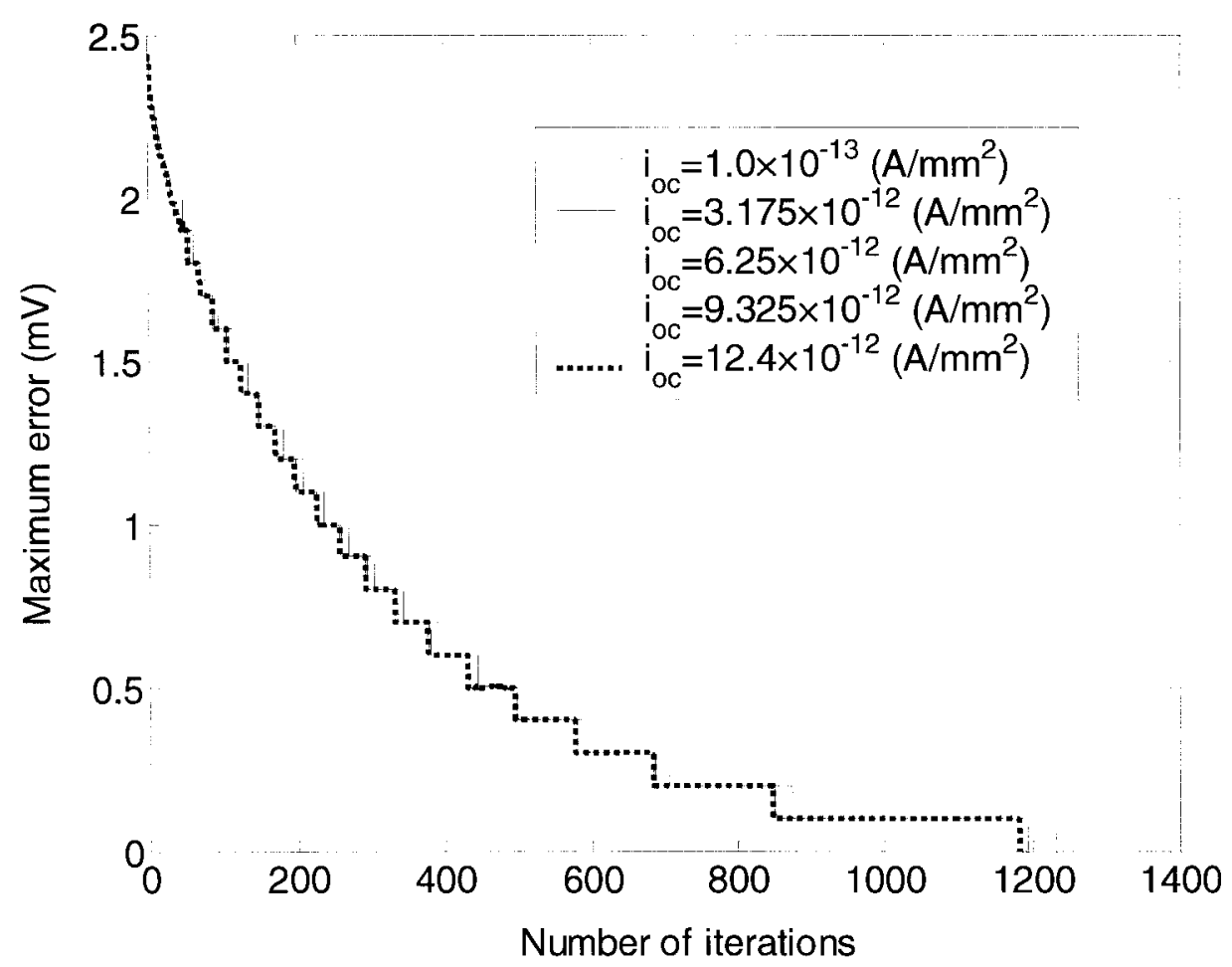

Fig. 6.27: The effect of $i_{o c}$ on convergence rate $(\mathrm{A} / \mathrm{C}=1.0)$ 


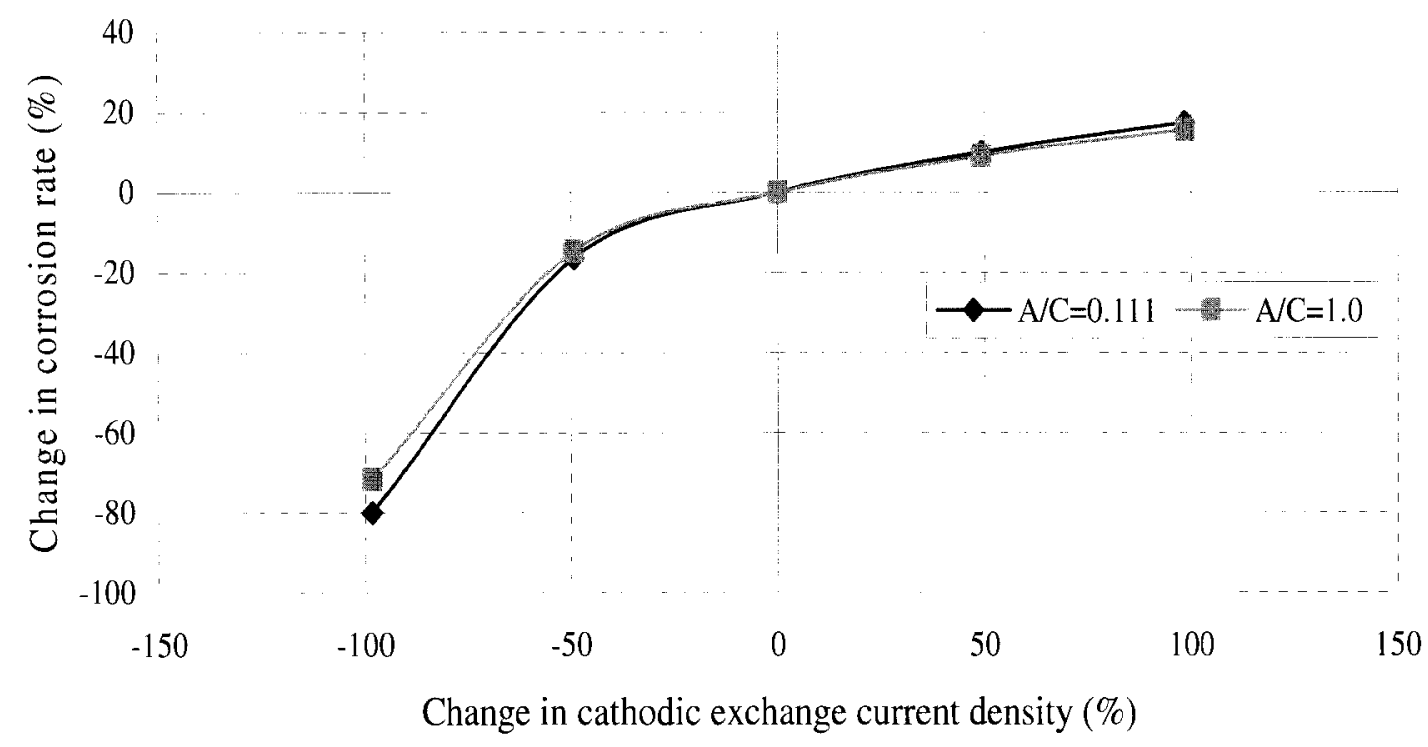

Fig. 6.28: The change in corrosion current rate vs. the cathodic exchange current density

\subsection{Investigation of the anodic half cell potential}

The effect of anodic half cell potential (of iron oxidation), $E_{o a}$, on corrosion rate is studied by varying it from -640 to $-820 \mathrm{mV}$, as measured against a standard calomel electrode. This range representative of the variation we observe in the corrosion literature. The anodic half cell potential of the base case is taken as $-780 \mathrm{mV}$. The results of the analysis for $\mathrm{A} / \mathrm{C}=0.111$ and $\mathrm{A} / \mathrm{C}=1.0$ are shown in Figs. 6.29-6.31 and Figs. 6.32 -6.33, respectively.

As illustrated in Figs. 6.29 and 6.30 , for $\mathrm{A} / \mathrm{C}=0.111$, the change in $E_{o a}$ primarily affects potential distribution along the anodic region. It can be observed in Fig. 6.29 that, as $E_{o a}$ becomes more negative, the potential difference between the anodic and cathodic regions increase, and this results in higher corrosion rates, as it can be observed in Fig. 6.30. As in previous cases, the change in $E_{\iota c}$ did not have a significant influence on the 
convergence rate of the numerical solution, as shown in Fig. 6.31. As illustrated in Figs. 6.32-6.34, similar qualitative observations can be made for $\mathrm{A} / \mathrm{C}=1.0$.

The change in corrosion rate vs. the change in $E_{o a}$ for both $\mathrm{A} / \mathrm{C}$ ratios is plotted in Fig. 6.35. The corrosion rate in this plot is also taken as the average current density in the anodic region. As it can be seen from this figure, the relationship between the two variations is linear with very similar curves, signifying that the effect of $E_{o a}$ on the corrosion rate is not a function of $\mathrm{A} / \mathrm{C}$ ratio. It should be noted from this figure that the effect of changes in $E_{o a}$ on corrosion rate is quite significant, with a slope greater than 3: a $5 \%$ change in $E_{o a}$ from the base case may result in $15 \%$ change in corrosion rate. It can be stated that $E_{\partial o}$ is a very sensitive parameter in corrosion modelling in concrete.

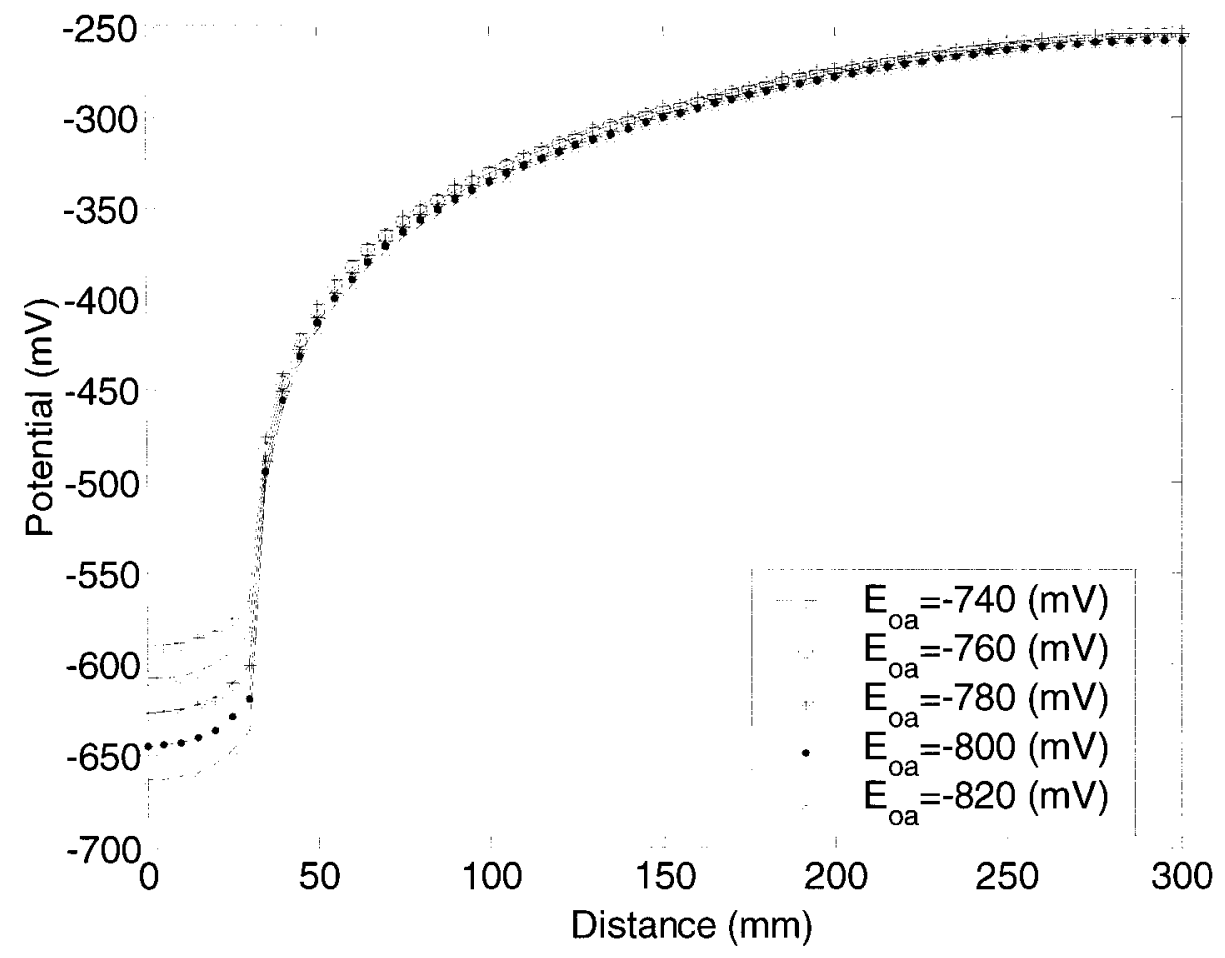

Fig. 6.29: The effect of $E_{o c}$ on potential distribution $(\mathrm{A} / \mathrm{C}=0.111)$ 


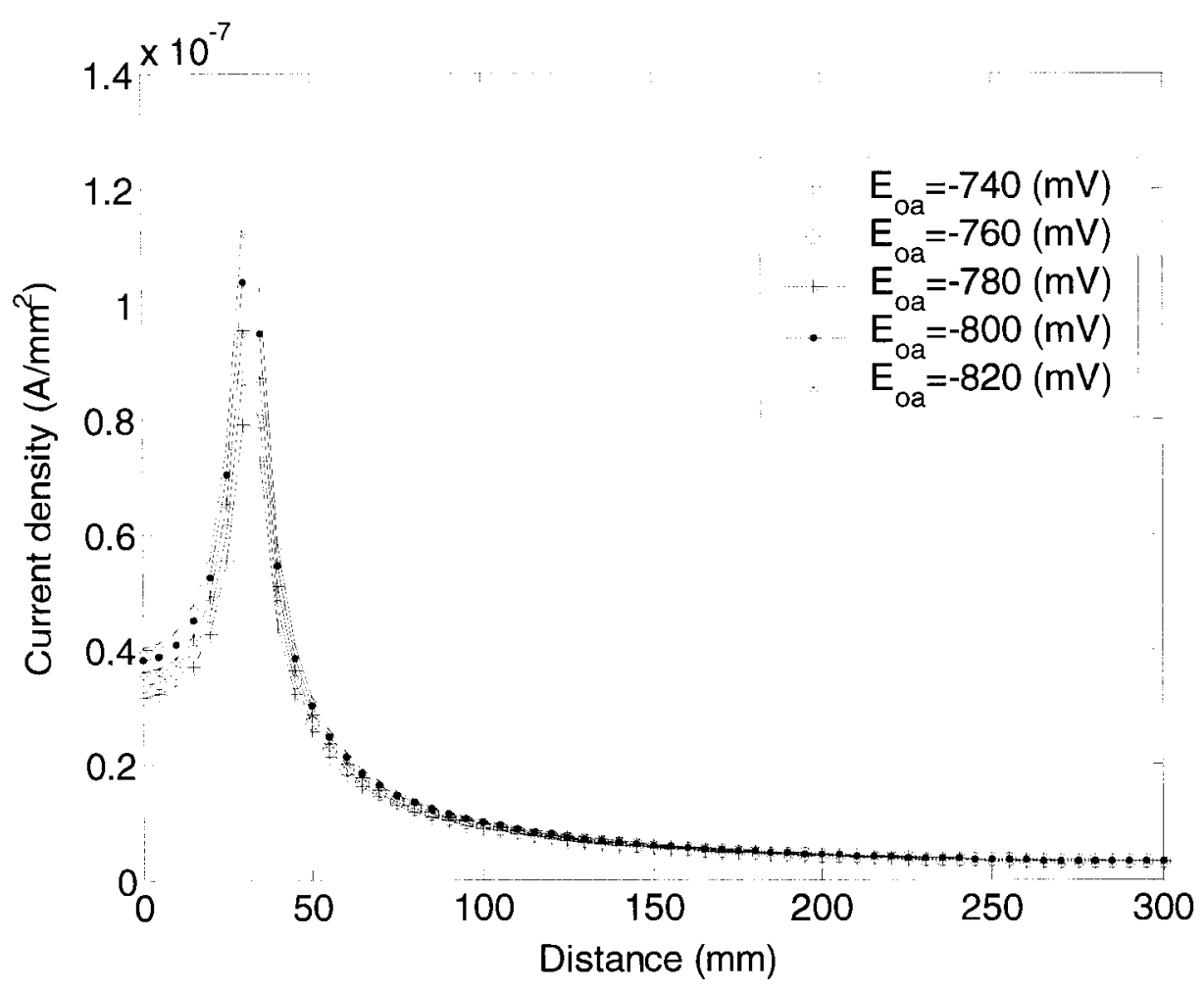

Fig. 6.30: The effect of $E_{o a}$ on current density $(\mathrm{A} / \mathrm{C}=0.111)$

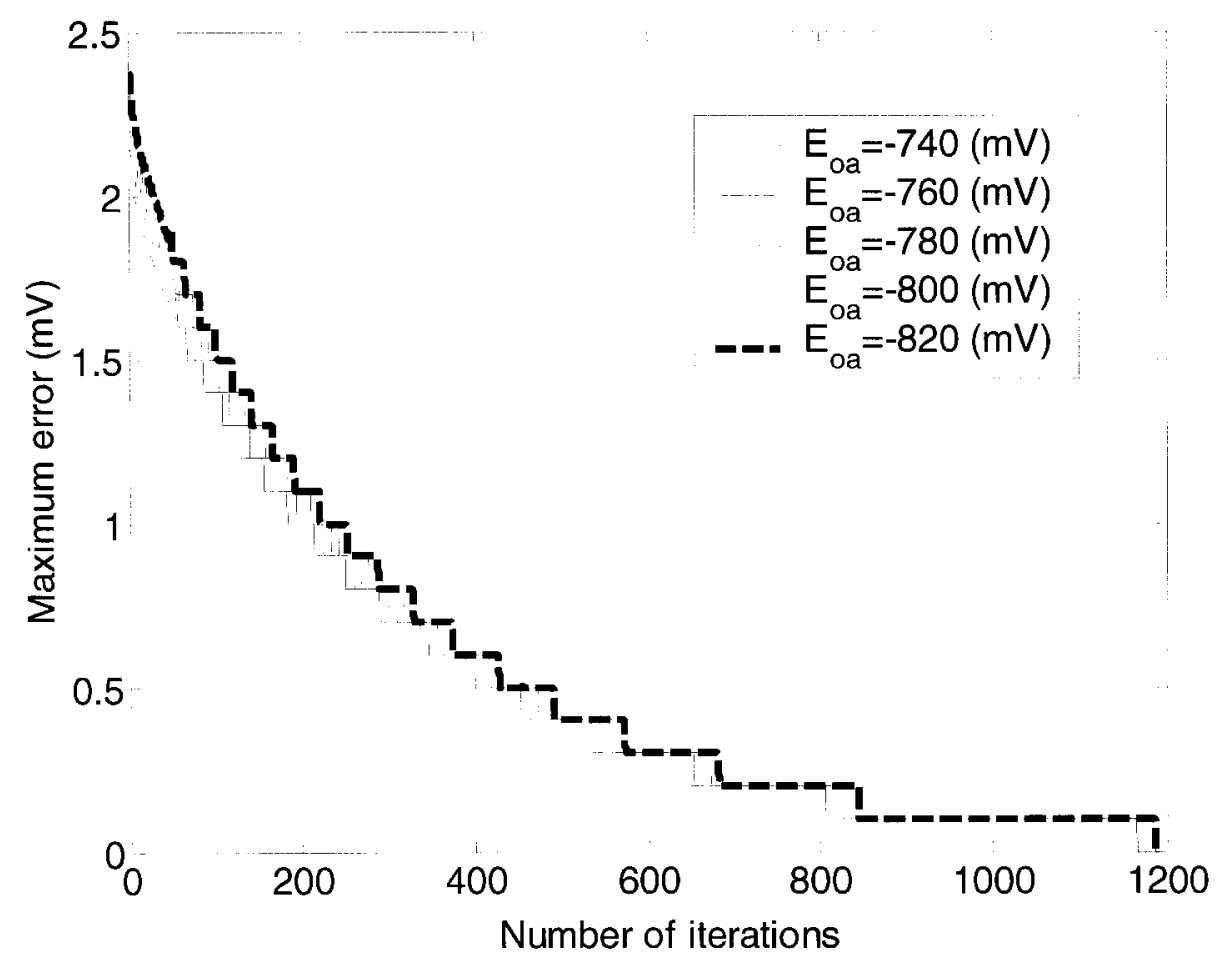

Fig. 6.31: The effect of $E_{o a}$ on convergence rate $(\mathrm{A} / \mathrm{C}=0.111)$ 


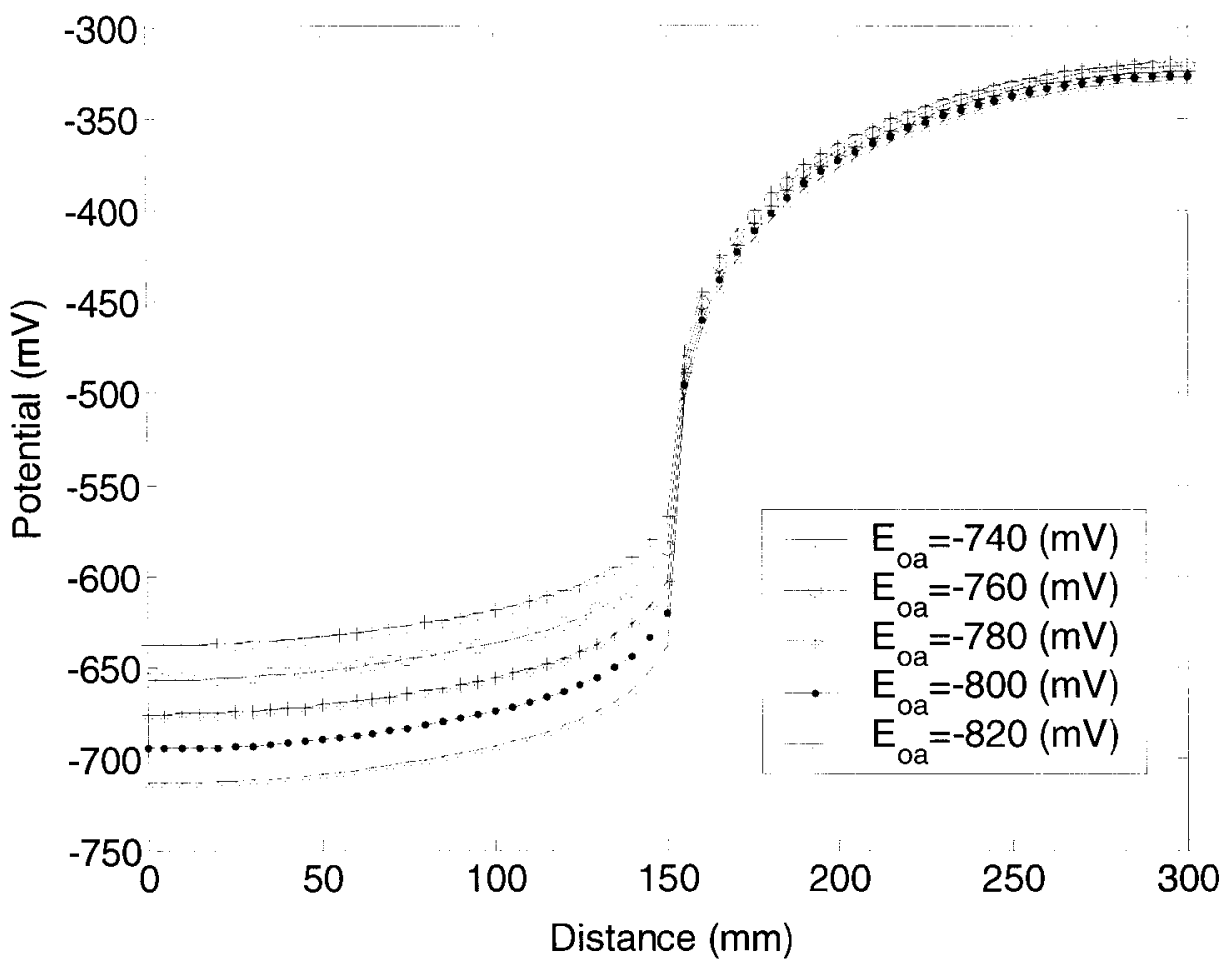

Fig. 6.32: The effect of $E_{o \iota l}$ on potential distribution $(\mathrm{A} / \mathrm{C}=1.0)$

$1.4^{\times 10^{-7}}$

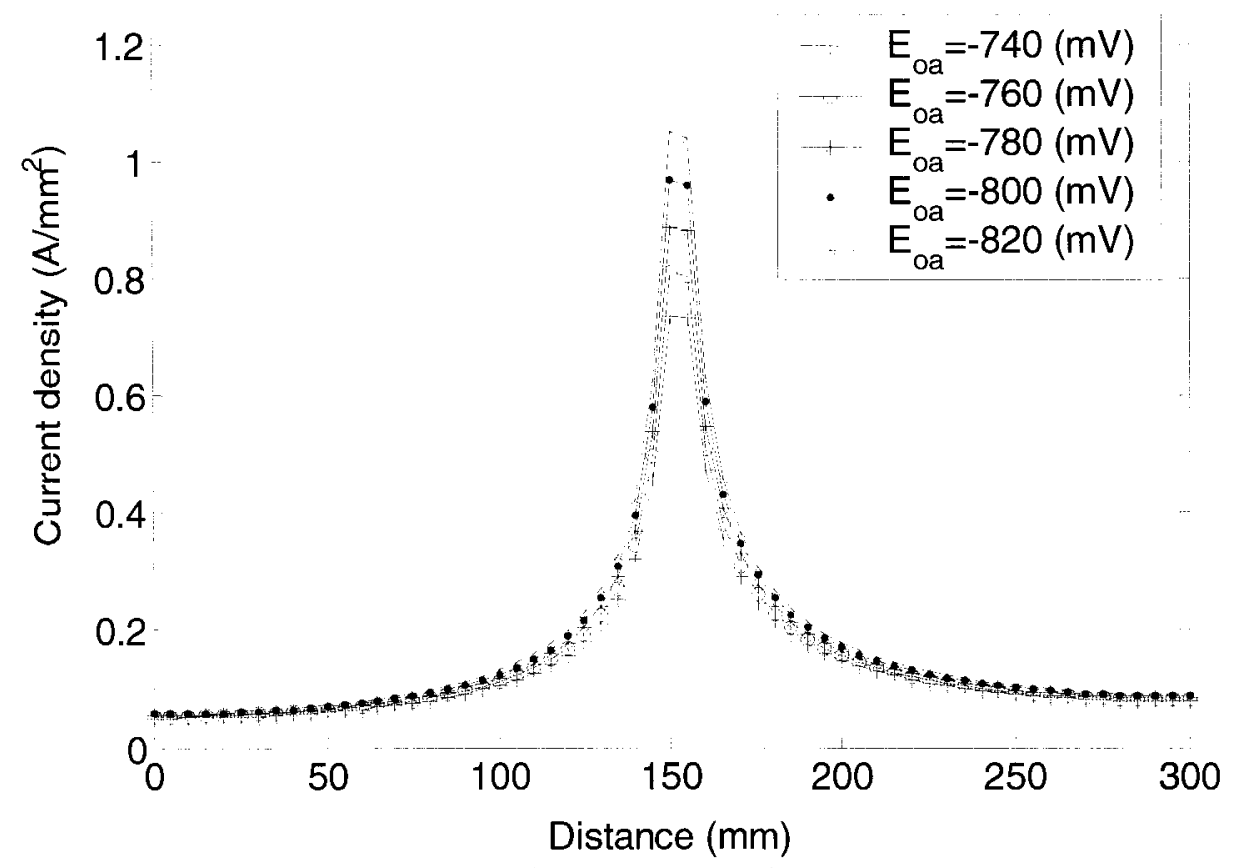

Fig. 6.33: The effect of $E_{o c}$ on corrosion current $(\mathrm{A} / \mathrm{C}=1.0)$ 


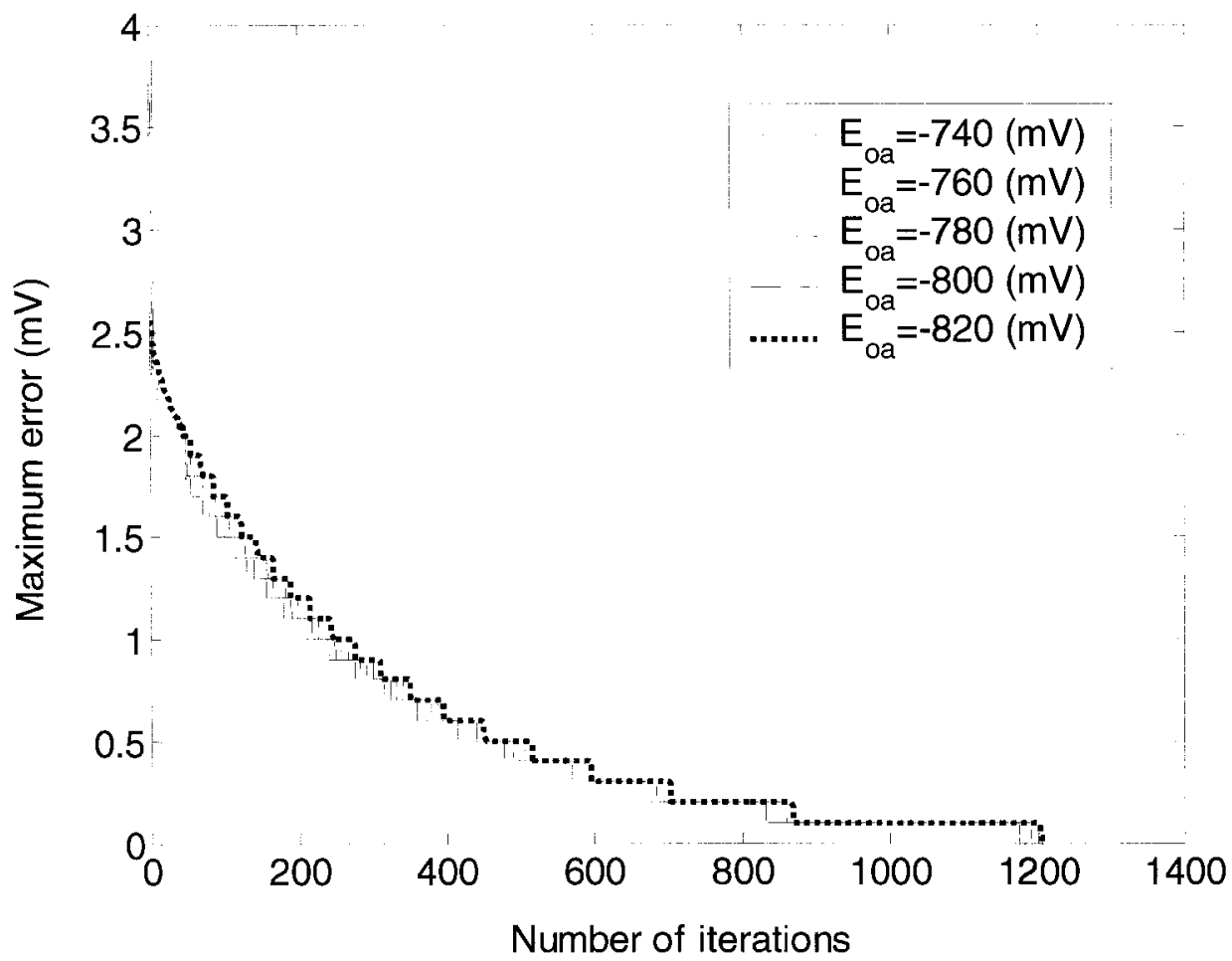

Fig. 6.34: The effect of $E_{o a}$ on convergence rate $(\mathrm{A} / \mathrm{C}=1.0)$

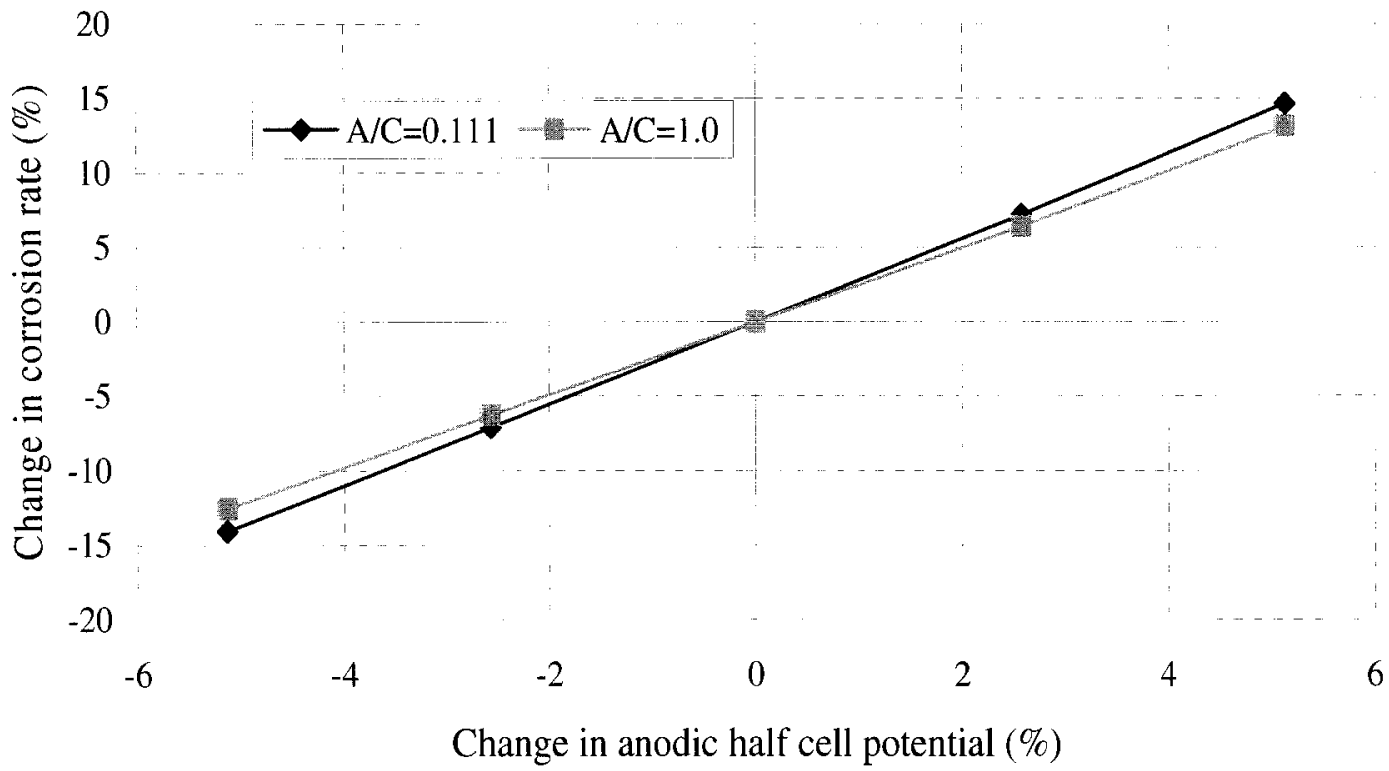

Fig. 6.35: The change in corrosion current rate vs. the anodic half cell potential 


\subsection{Investigation of the cathodic half cell potential}

The effect of cathodic half cell potential (of oxygen reduction), $E_{o c}$, on corrosion rate is studied by varying it from 120 to $200 \mathrm{mV}$, as measured against a standard calomel electrode, by $20 \mathrm{mV}$ increments. This range representative of the variation we observe in the corrosion literature. The anodic half cell potential of the base case is taken as $160 \mathrm{mV}$. The results of the analysis for $\mathrm{A} / \mathrm{C}=0.111$ and $\mathrm{A} / \mathrm{C}=1.0$ are shown in Figs. 6.36-6.38 and Figs. 6.39-6.41, respectively.

As illustrated in Figs. 6.36 and 6.37, for $\mathrm{A} / \mathrm{C}=0.111$, the change in $E_{o c}$ primarily affects potential distribution along the cathodic region. It can be observed in Fig. 6.36 that, as $E_{o c}$ becomes larger, the potential difference between the anodic and cathodic regions increase, and this results in higher corrosion rates, as it can be observed in Fig. 6.37. As in previous cases, the change in $E_{o c}$ did not have a significant influence on the convergence rate of the numerical solution, as shown in Fig. 6.38. As illustrated in Figs. 6.39-6.41, similar qualitative observations can be made for $\mathrm{A} / \mathrm{C}=1.0$.

The change in corrosion rate vs. the change in $E_{o c}$ for both $\mathrm{A} / \mathrm{C}$ ratios is plotted in Fig. 6.42. The corrosion rate in this plot is also taken as the average current density in the anodic region. As it can be seen from this figure, the relationship between the two variations is linear with very similar curves, signifying that the effect of $E_{o c}$ on the corrosion rate is not a function of $\mathrm{A} / \mathrm{C}$ ratio. It should be noted from this figure that the effect of changes in $E_{o c}$ on corrosion rate is not as significant as the effect of $E_{o a}$ : a 10 
$\%$ change in $E_{o c}$ from the base case may result in $5 \%$ change in corrosion rate.

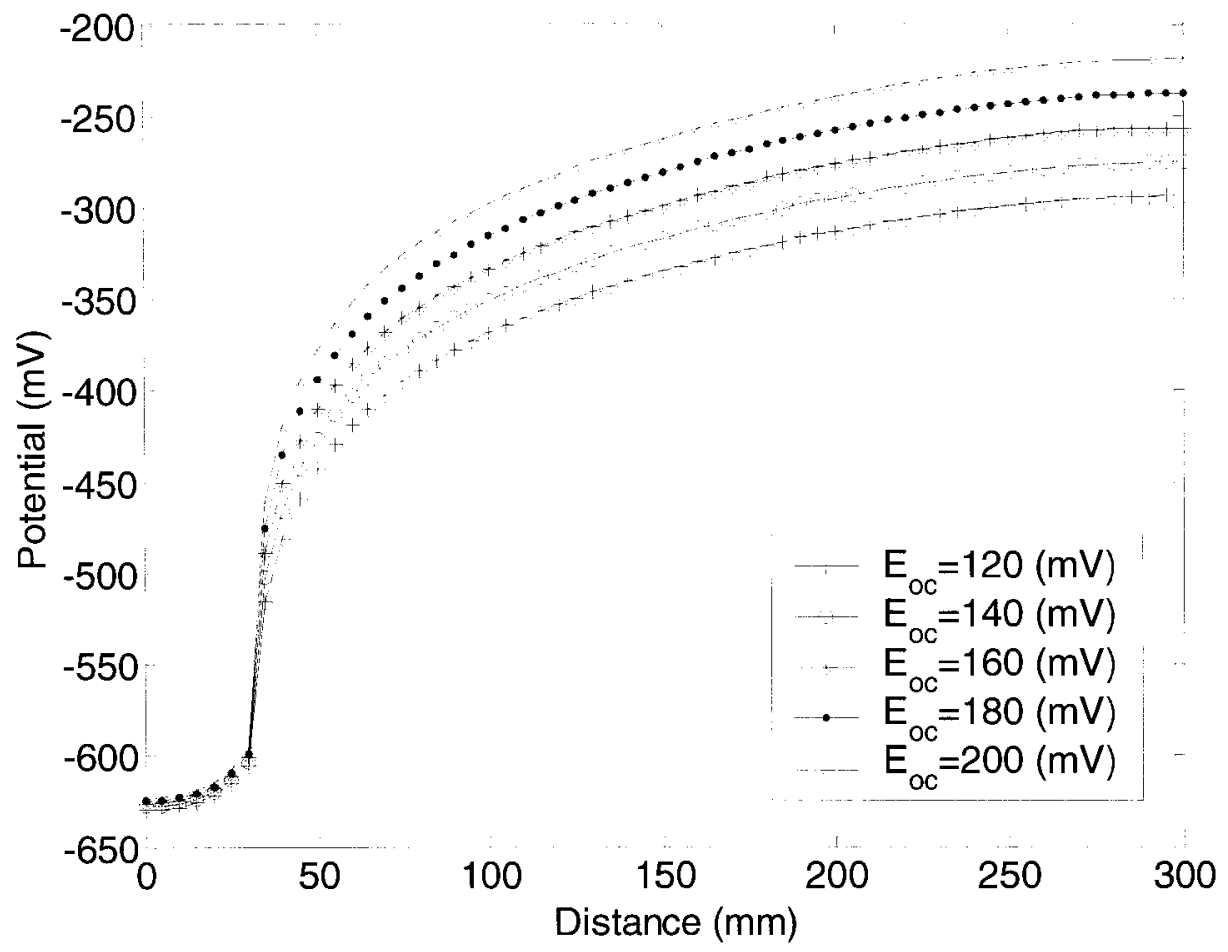

Fig. 6.36: The effect of $E_{o c}$ on potential distribution $(\mathrm{A} / \mathrm{C}=0.111)$ $1.4^{\times 10^{-7}}$

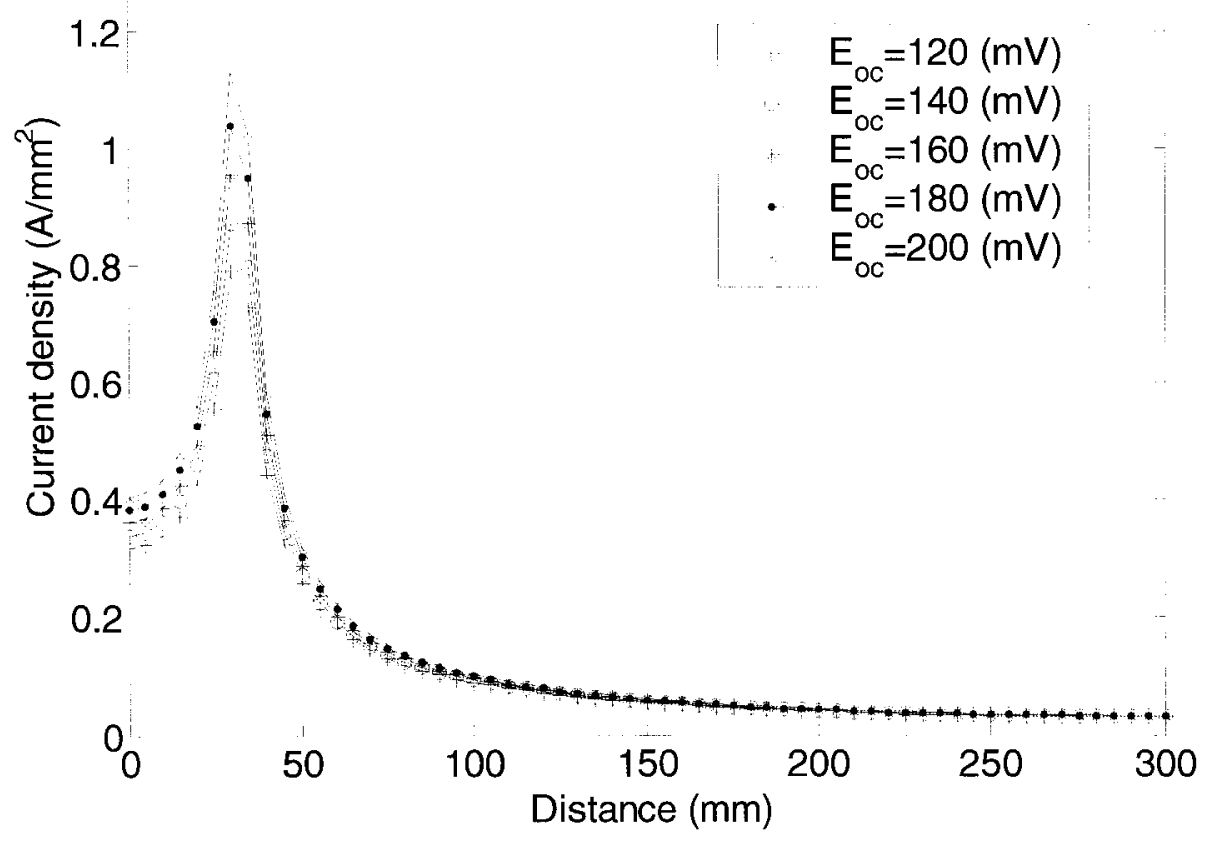

Fig. 6.37: The effect of $E_{o c}$ on current density $(\mathrm{A} / \mathrm{C}=0.111)$ 


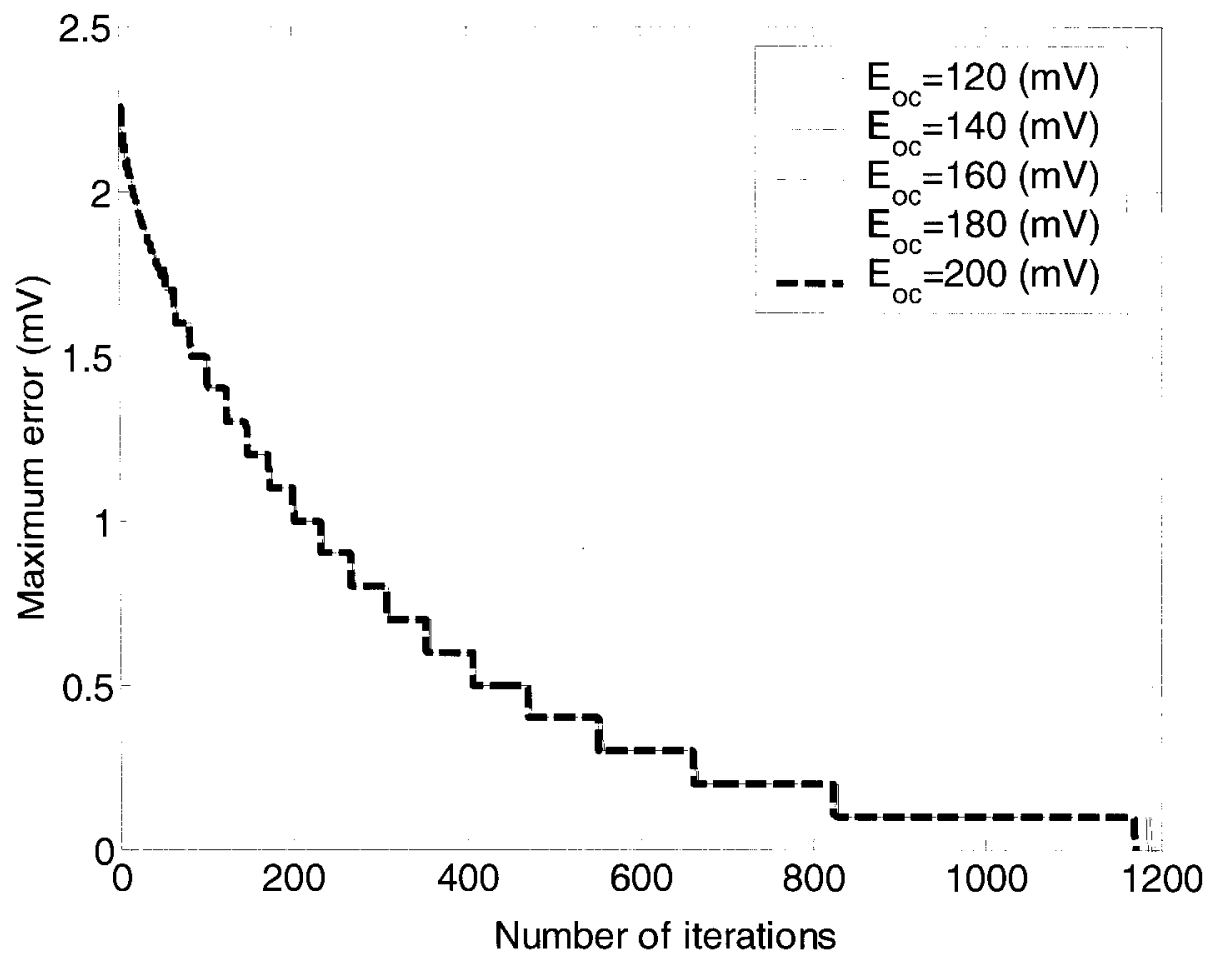

Fig. 6.38: The effect of $E_{o c}$ on convergence rate $(\mathrm{A} / \mathrm{C}=0.111)$

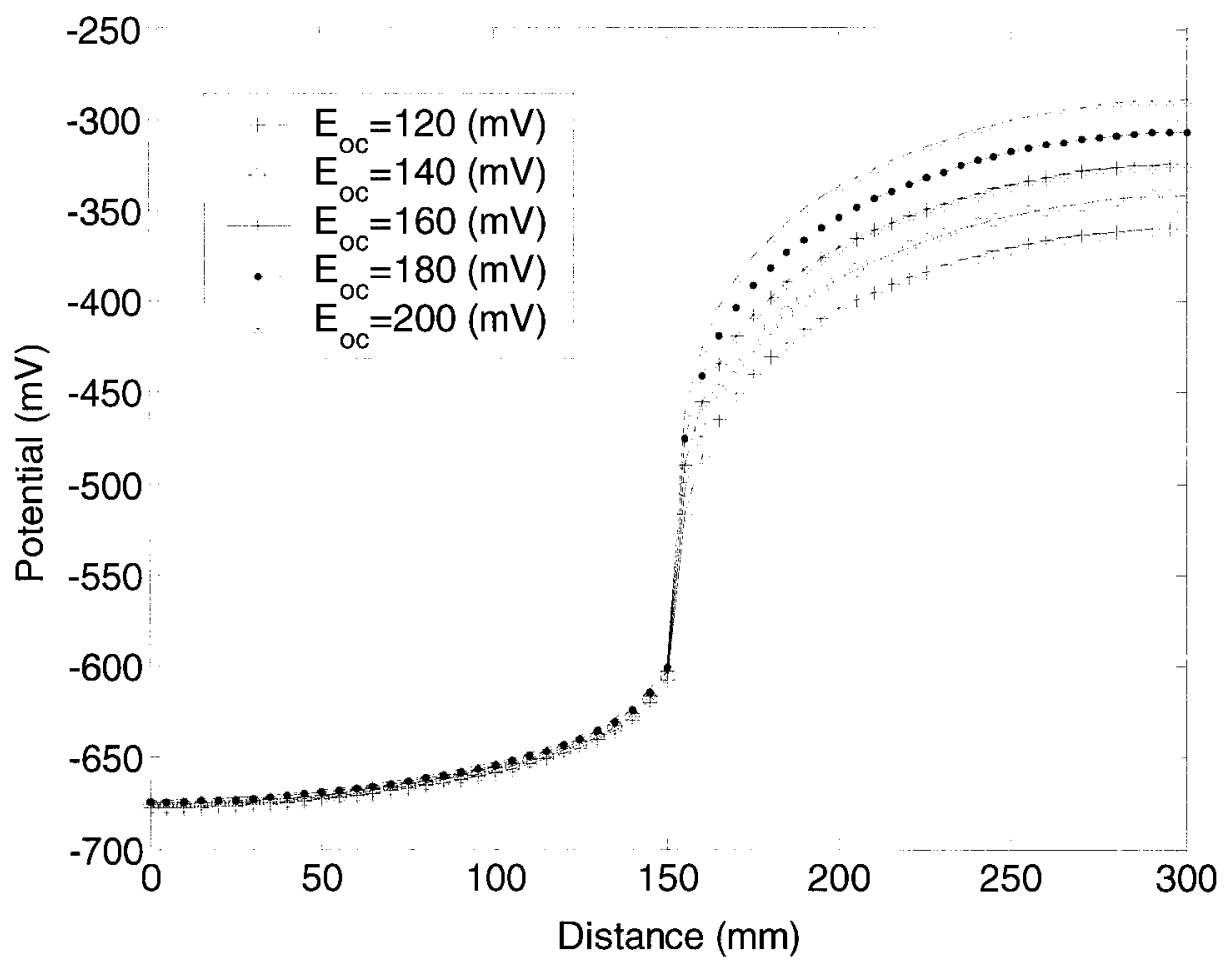

Fig. 6.39: The effect of $E_{o c}$ on potential distribution $(\mathrm{A} / \mathrm{C}=1.0)$ 


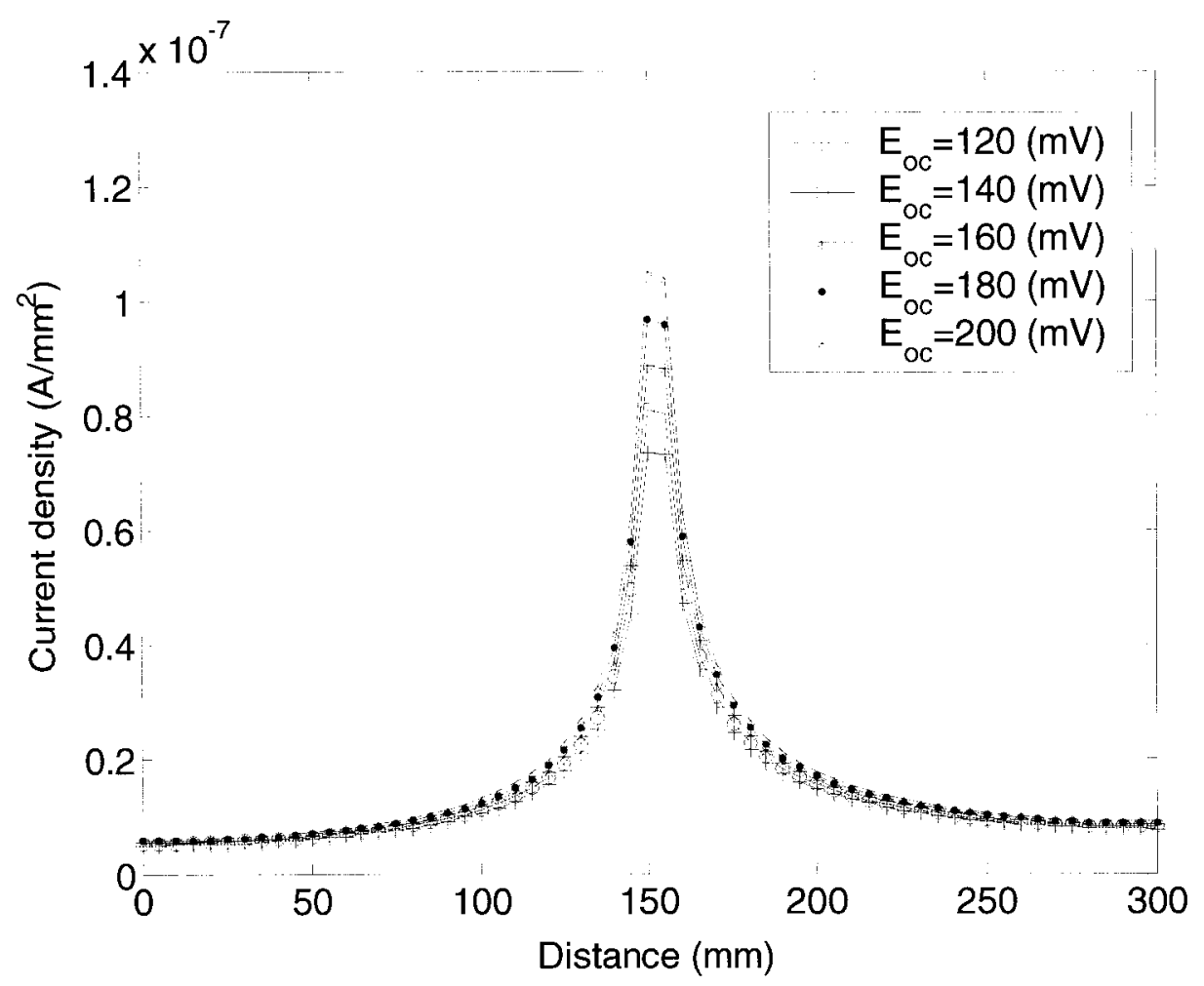

Fig. 6.40: The effect of $E_{o c}$ on current density $(\mathrm{A} / \mathrm{C}=1.0)$

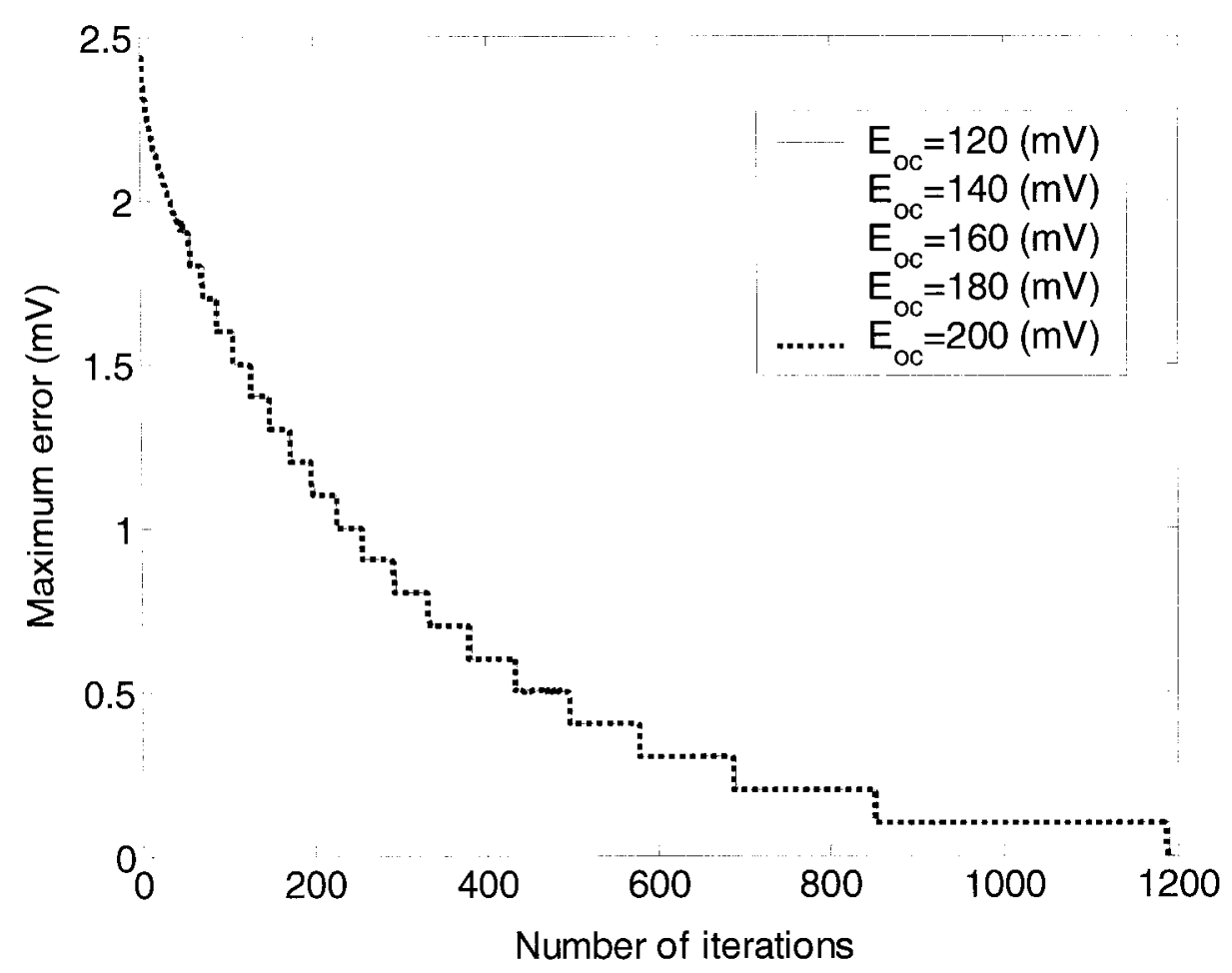

Fig. 6.41: The effect of $E_{o c}$ on convergence rate $(\mathrm{A} / \mathrm{C}=1.0)$ 


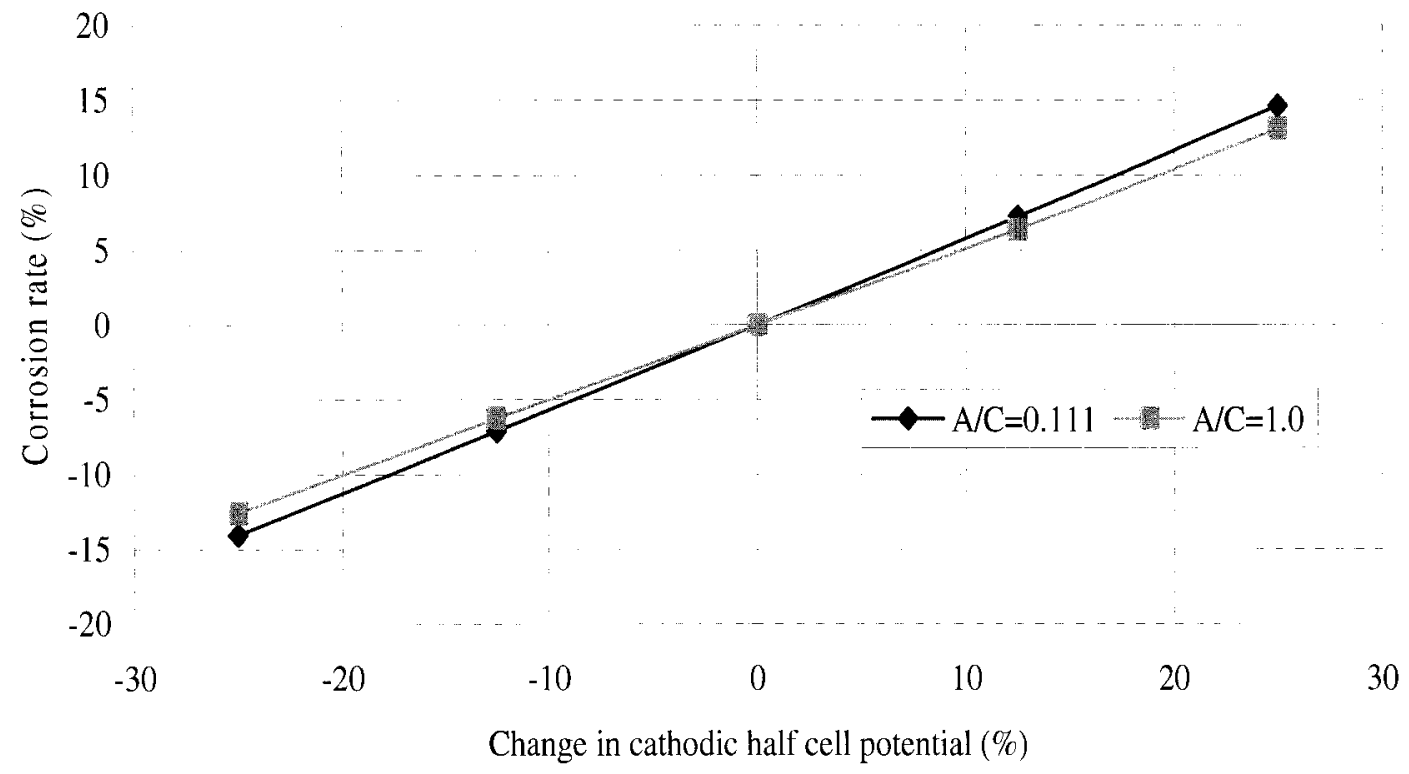

Fig. 6.42: The change in corrosion current rate vs. the cathodic half cell potential

\subsection{Summary of the results}

The summary of the investigation presented in this chapter is presented in Figs. 6.43-6.46.

The following observations can be made from these figures:

- When the parameters are varied between $-50 \%$ to $+50 \%$ from the base case, the variation in corrosion rate follows a linear pattern.

- For anodic and cathodic exchange current densities, the linearity continues when they are varied beyond $+50 \%$ from the base case. However, as they become smaller (i.e. variation from the base case less than $-50 \%$ ), the corrosion rate changes nonlinearly, with an accelerating pattern.

- $\mathrm{A} / \mathrm{C}$ ratio does not have a significant influence on the change in corrosion rate as the corrosion parameters are varied. The only exception for this observation is the case of the anodic Tafel slope; there is a recognizable difference between the changes in 
corrosion rate for two $\mathrm{A} / \mathrm{C}$ ratios when the anodic Tafel slope is varied. In general, it is observed that cases with $\mathrm{A} / \mathrm{C}=0.111$ are more affected by the changes in corrosion parameters than the cases with $\mathrm{A} / \mathrm{C}=1.0$.

- Within the linear region of the curves in Figs. 6.43 and 6.45 , anodic half cell potential, $E_{o a}$, and cathodic Tafel slope, $\beta_{c}$, have the largest absolute slopes. A summary of the slopes for both $\mathrm{A} / \mathrm{C}$ ratios are presented in Figs. 6.44 and 6.46. This implies that corrosion models are more sensitive to changes in these parameters than the others.

- Anodic Tafel slope, $\beta_{a}$, and the cathodic half cell potential, $E_{o c}$, also play important role in corrosion modelling albeit not as much as cathodic Tafel slope, $\beta_{c}$, and anodic half cell potential, $E_{o d}$.

- Within the linear region of the curves in Figs. 6.43 and 6.45 , the change in exchange current densities is not a significant factor on the corrosion rate of steel in concrete. However, in the nonlinear zone both exchange current densities, especially, $i_{o c}$, become important. 


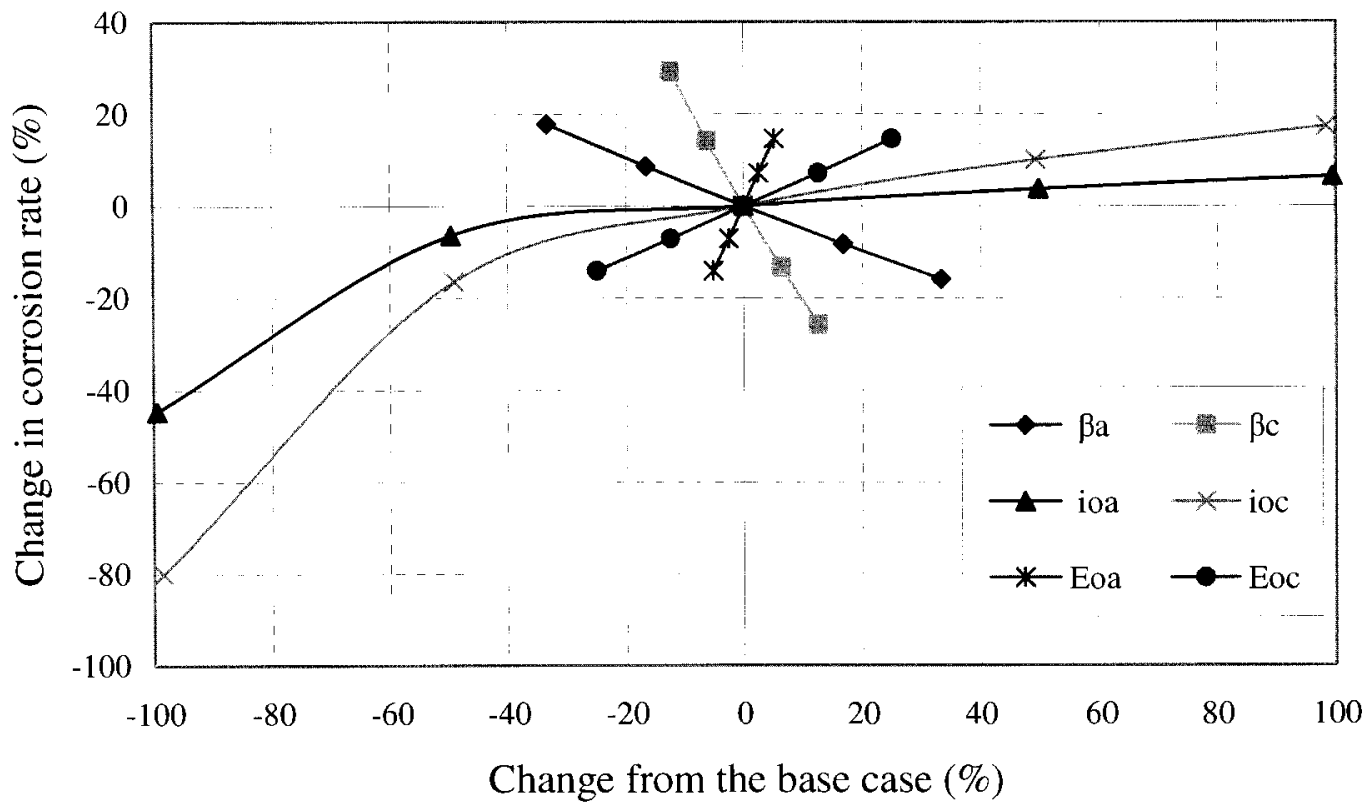

Fig. 6.43: Summary of the effects of corrosion parameters on corrosion rate $(\mathrm{A} / \mathrm{C}=$ 0.111 )

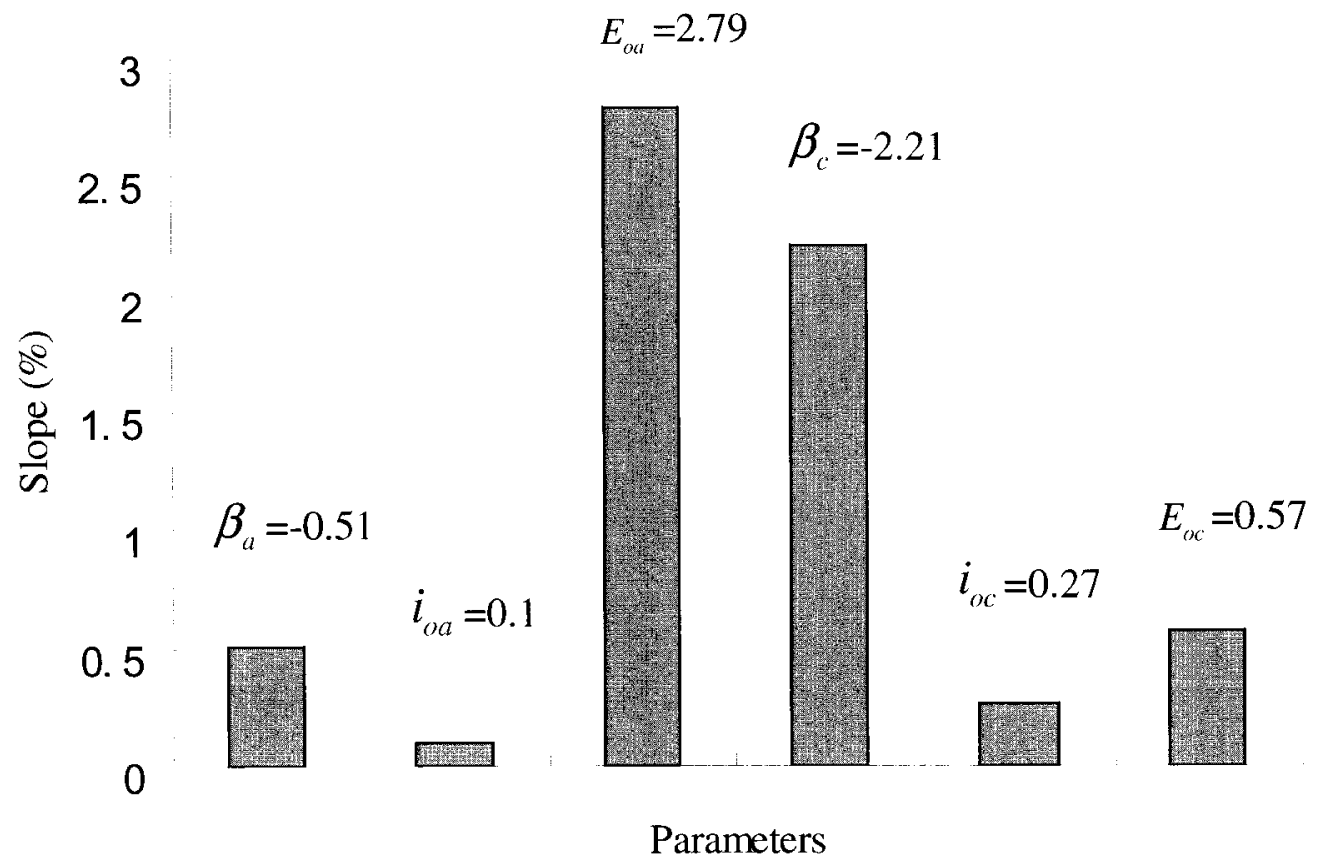

Fig. 6.44: Comparison of the effect of each parameters on corrosion rate using the slopes of the linear portion of the change curves $(\mathrm{A} / \mathrm{C}=0.111)$ 


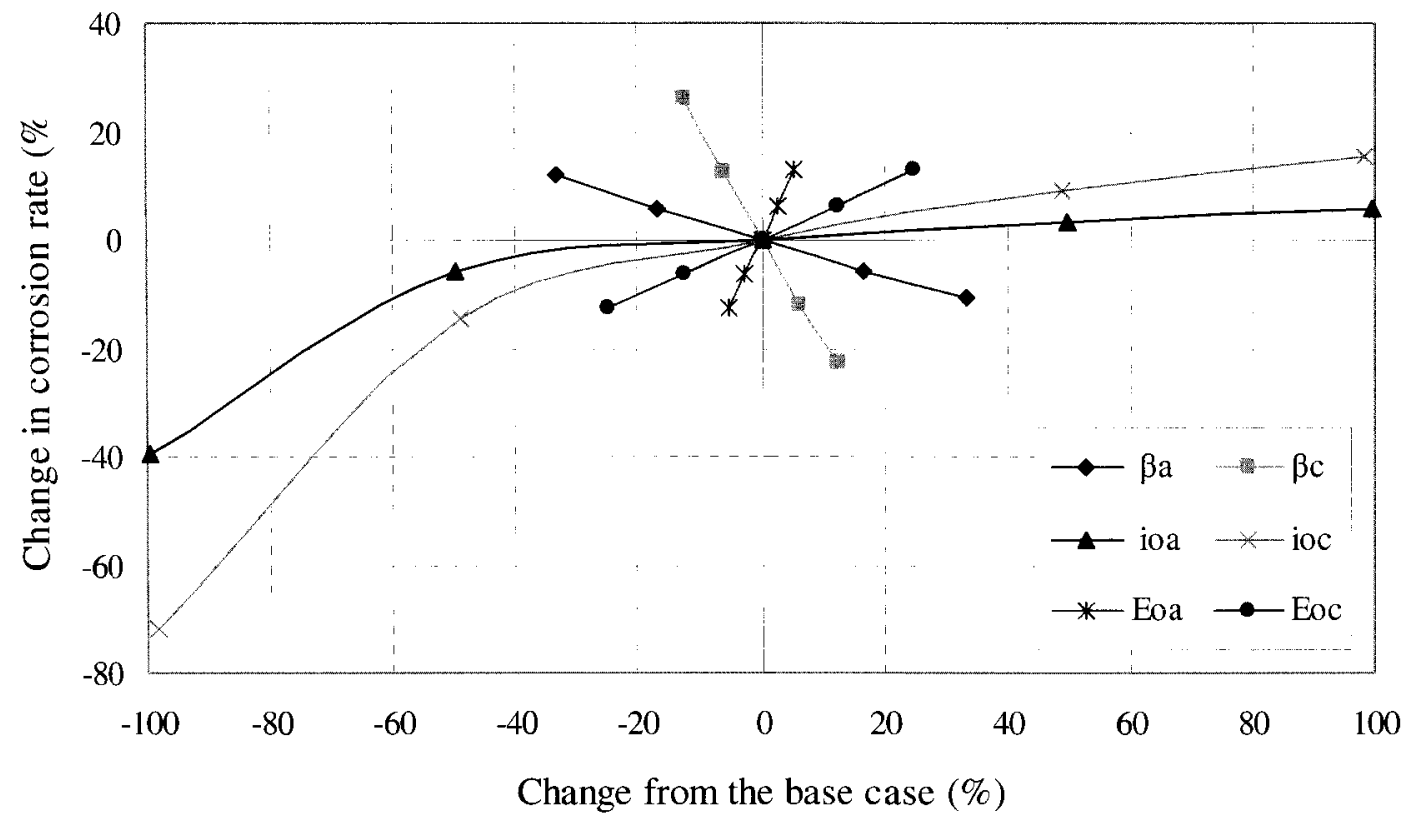

Fig. 6.45: Summary of the effects of corrosion parameters on corrosion rate $(A / C=1.0)$

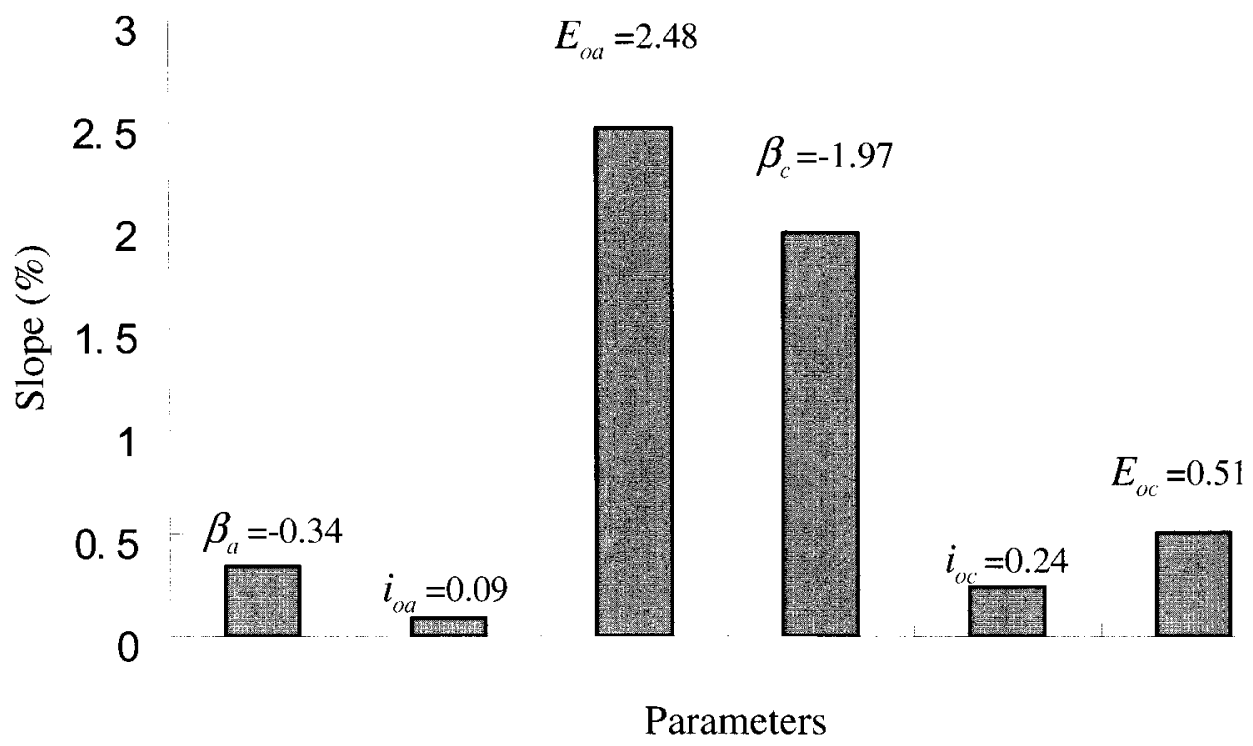

Fig. 6.46: Comparison of the effect of each parameters on corrosion rate using the slopes of the linear portion of the change curves $(\mathrm{A} / \mathrm{C}=1.0)$ 


\section{Chapter 7}

\section{Conclusions and Future Work}

\subsection{Summary}

A two-dimensional nonlinear finite element technique was developed to model steel corrosion in concrete members. The nonlinear solution algorithm was implemented as a Windows-based computer program using Visual Basic.Net 2003. The program uses a finite element engine that was previously developed at Carleton University, CONDUR, to solve Laplace's equation. With the new approach, the convergence rate and the stability of the nonlinear solution are significantly improved. The sensitivity of the results and convergence of the solution with respect to element size, anode-to-cathode ratio and concrete resistivity are investigated. The effect of the selection of corrosion parameters on the solution is also studied.

\subsection{Conclusions}

The study presented in this research yielded the following conclusions:

- The nonlinear solution of the Laplace's equation for corrosion of steel in concrete can be difficult due to convergence problems originating from the non-linear polarization boundary conditions. 
- The DIM has limitations in solving the steel corrosion problem in concrete. The convergence process in the DIM shows a random pattern, and the results obtained from the DIM show significant fluctuations.

- The issues with the DIM exist for all element sizes, $\mathrm{A} / \mathrm{C}$ ratios and concrete resistivities; therefore their origin is numerical.

- The use of an under-relaxation factor is necessary to eliminate the oscillations in the solution along the cathodic site and to reach reasonable levels of convergence.

- For cases in which the concentration polarization plays an important role, the use of a liming current density control factor is essential to reach convergence.

- The MDIM, through under-relaxation and limiting current density correction factors, correct most issues encountered in the DIM.

- The choice of the under-relaxation factor affects only the convergence rate, but does not have a significant impact on the corrosion rate predictions.

- For very small element sizes the MDIM show inaccuracies in results and convergence problems. For converged cases, the element size does not have a significant effect on convergence rate.

- The numerical solution by the MDIM shows some fluctuations and inaccuracies as the $\mathrm{A} / \mathrm{C}$ ratio becomes very small (i.e. less than 0.1 ). The main reason for these inaccuracies is that for small $\mathrm{A} / \mathrm{C}$ ratios, the anode length is relatively small, and the element size used for these cases is not small enough to capture the effect of the anode. Therefore, the investigation of the solution of very small $\mathrm{A} / \mathrm{C}$ ratios requires further investigation. Therefore, in selecting the element size, a fine balance between the accuracy and convergence should be found.

- The MDIM is quite successful in solving $\mathrm{A} / \mathrm{C}$ ratios larger than 0.074 for all limiting 
current densities.

- $\mathrm{A} / \mathrm{C}$ ratio does not have a significant effect on the convergence rate.

- Concrete resistivity affects the results and the convergence rate of the MDIM. The potential difference between anode and cathode becomes larger as the resistivity increases; however, the current density decreases with increasing resistivity, even though the potential gradient becomes larger.

- As concrete resistivity becomes smaller (i.e. more conductive concrete), it is observed that achieving convergence is harder.

- When corrosion parameters are varied between $-50 \%$ to $+50 \%$ from the base case, the variation in corrosion rate follows a linear pattern.

- For anodic and cathodic exchange current densities, the linearity continues when they are varied beyond $+50 \%$ from the base case. However, as they become smaller (i.e. variation from the base case less than $-50 \%$ ), the corrosion rate changes nonlinearly, with an accelerating pattern.

- $\mathrm{A} / \mathrm{C}$ ratio does not have a significant influence on the change in corrosion rate as the corrosion parameters are varied. The only exception for this observation is the case of the anodic Tafel slope; there is a recognizable difference between the changes in corrosion rate for two $\mathrm{A} / \mathrm{C}$ ratios when the anodic Tafel slope is varied. In general, it is observed that cases with $\mathrm{A} / \mathrm{C}=0.111$ are more affected by the changes in corrosion parameters than the cases with $\mathrm{A} / \mathrm{C}=1.0$.

- Within the linear region of variation, anodic half cell potential, $E_{o c}$, and cathodic Tafel slope, $\beta_{c}$, have the largest absolute slopes. This implies that corrosion models are more sensitive to changes in these parameters than the others. 
- Anodic Tafel slope, $\beta_{a}$, and the cathodic half cell potential, $E_{o c}$, also play an important role in corrosion modelling albeit not as much as cathodic Tafel slope, $\beta_{c}$, and anodic half cell potential, $E_{o a}$.

- Within the linear region of variation, the change in exchange current densities is not a significant factor on the corrosion rate of steel in concrete. However, in the nonlinear zone both exchange current densities, especially, $i_{o c}$, become important.

\subsection{Future work}

The following recommendations for future work can be made:

- The solution of cases with very small $\mathrm{A} / \mathrm{C}$ ratios $(\mathrm{A} / \mathrm{C}<0.03)$ should be studied further. The use of higher-order elements should be investigated for improving convergence for these cases.

- The effect of mixed Neumann and Dirichlet boundary conditions on the convergence processes should be investigated.

- The difference in results and convergence rate between the method proposed in this research and the methods that are based on the linearization of the boundary conditions (e.g. by using Taylor's series expansion) needs to be analyzed. A cost-benefit analysis would be useful to assess the computation time vs. accuracy. This may require an experimental study to compare two approaches. 


\section{References}

Balabanic, G., Bicanic, N., Durekovic, A. (1996). "The influence of w/c ratio, concrete cover thickness and degree of water saturation on the corrosion rate of reinforcing steel in concrete," Cement and Concrete Research, Vol.26, No.5, pp. 761-769.

Bentur, A., Diamond, S. and Berke, N.S. (1997). Steel corrosion in concrete, E\&FN spon, London.

Bockris, J. and Reddy, A., (1997). Modern Electrochemistry, Plenum \& Rosetta, New York.

Botte, V., Mansutti, D., Pascarelli, A. (2005). "Numerical modeling of iron corrosion due to an acidic aqueous solution," Applied Numerical Mathematics, Vol.55, pp. 253-263.

Broomfield, J. P. (1997). Corrosion of steel in concrete, E\&FN Spon, London and New York.

Broomfield, J.P. (2000). "The principles and practice of galvanic cathodic protection for reinforced concrete structures," CPA Monograph No. 6.

Chen, R.-C. and Liu, J-L (2002). "Monotone iterative methods for the adaptive finite element solution of semiconductor equations," Journal of Computational and Applied Mathematics Vol.159, pp.341-364.

Devereux, O. F. and Yeum, K. S. (1992). "Modeling electron transfer, Diffusional effects, passivation, and ohmic loss in multi-reaction polarization curves," Computer Modeling in Corrosion. ASTM STP 1154, pp. 126-142.

Dhatt, G., Touzot, G. (1984). The finite element method displayed, J. Wiley \& Sons, N, 
New York.

G. Eliezer (1993). Electrode kinetics, VCH Publishers Inc., New York.

Elsener, B. (2005). "Corrosion rate of steel in concrete-measurements beyond the Tafel law," Corrosion science, Vol.47, pp. 3019-3033.

Fadayomi, J. (1997). "Corrosion inhibitors," Concrete, Vol.31, pp. 21-22.

Feliu, S. Gonzalez, J., Andrade, C. (1995). "Effect of current distribution on corrosion rate measurements in reinforced concrete," Corrosion, Vol.51, pp. 79-90.

Garces, P., Andrade, M.C., Saez, A., Alonso, M.C., (2005). "Corrosion of reinforceing steel in neutral and acid solutions simulating the electrolytic environments in the micropores of concrete in the propagation period," Corrosion Science Vol.47, pp.289-306.

Ghods, P., Isgor, O.B., Pour-Ghaz, M. (2006). "A practical method for calculating the corrosion rate of uniformly depassivated reinforcing bars in concrete," Materials and Corrosion. Accepted paper.

Gulikers, J. (2005a). "Theoretical considerations on the supposed linear relationship between concrete resistivity and corrosion rate of steel reinforcement," Materials and Corrosion Vol.56, No. 6, pp. 393-403.

Gulikers, J. (2005b). "Numerical modelling of reinforcement corrosion in concrete," Corrosion in reinforced concrete structures," Edited by Hans Bohni, Corrosion in reinforced concrete structures, Woodhead publishing limited, Cambridge, UK.

Glasstone, S. (1942). "principles of electrochemistry," Van Nostrand, New York

Harker, A., Sharland, S., and Tasker, P. (1987). "A mathematical model of uniform corrosion of intermediate level radioactive waste canisters in concrete," Radioactive Waste Management and the Nuclear Fuel Cycle, Vol.8 No.1, pp 
65-85.

Hansen, E. J. and Saouma, V. E. (1999). "Numerical simulation of reinforced concrete deterioration: part II-steel corrosion and concrete cracking," ACI materials Journal, Vol.96, No. 3, pp. 331-338.

Hassanein, A.M, Glass, G. K., and Buenfeld, N.R. (1998). “A mathematical model for electrochemical removal of chloride from concrete structures," Corrosion, Vol.54, No.4, pp. 323-332.

Hines, J.G. (1982). “Analysis of complex polarization curves,” British Corrosion Journal, Vol.18, No.1, pp. 10-14.

Hope, B.B., Ihekwaba, N.M., and Hansson, C. M. (1995). "Influence of multiple rebar mats on electrochemical removal of chloride from concrete," Material Science Forum (192-194), pp. 883-890.

Isgor, O.B. (2001). “A durability model for chloride and carbonation induced steel corrosion in reinforced concrete members," Ph.D. Thesis, Carleton University, Canada.

Isgor, O.B., Razaqpur, A.G., (2004). "Finite element modeling of coupled heat transfer moisture transport and carbonation processes in concrete structure," Cement \&Concrete Composites, Vol. 26, pp. 57-73.

Isgor, O.B., Razaqpur, A.G. (2005). "Modelling steel corrosion in concrete structures," Materials and Structures, Vol.38, pp. 1359-5997.

Jaggi, S., Bohni, H. and Elsener, B. (2001). "Macrocell corrosion of steel in concreteexperiments and numerical modelling," Publ. Associazione Italiana Metallurgia, Milan on CD. 
Kranc, S.C. and Sagues, A.A. (1992). "Computation of corrosion macrocell current distribution and electrochemical impedance of reinforcing steel in concrete," Computer Modeling in Corrosion, edited by X.Y. Mann, ASTM International, Philadelphia, USA. pp.95-112.

Kranc, S.C. and Sagues A.A. (1993). "Polarization current distribution and electrochemical impedance response of reinforced concrete when using guard rig electrodes," Electrochimica Acta, Vol. 38, No.14, pp.2055-2061.

Kranc, S.C. and Sagues, A.A. (2001). "Detailed modeling of corrosion macrocells on steel reinforcing in concrete," Corrosion Science, Vol.43, pp.1355-1372.

Kytbe, P. K. (1995). Boundary element methods, University of New Orleans, CRC press, USA.

Liang, M-T, Jin, W-L, Yang, R-J, Huang, N-M (2004). "Prederminate model of corrosion rate of steel in concrete," Cement and Concrete Research, Vol. 35, pp.1827-1833.

Martin-Perez, B. (1999). "Service life modeling of R.C. Highway Structures Exposed to Chlorides," Ph.D. Thesis, University of Toronto, Canada.

Martin-Perez, B., Pantazopoulou, S.J., Thomas, M.D.A. (2001). "Numerical solution of mass transport equations in concrete structures," Computers and Structures Vol.79, pp.1251-1264.

Maruya, T., Hsu, K., Takeda, H., Tangtermsirikul, S. (2003). "Numerical modelling of steel corrosion in concrete structures due to chloride ion, oxygen and water movement," Journal of Advanced Concrete Technology, Vol.1, No.2, pp.147-160.

Matsushima, M., Tsutsumi, T., Seki, H., and Matsui, K. (1996). "Corrosion rate of reinforcement in concrete due to chloride attack," Edited by R. Dhir and M. Jones, Concrete Repair, Rehabilitation and Protection, London: E \& FN Spon, pp. 
235-244.

Mohammed, T. U., Otsuki, N. and Hisada, M. (1999). "Corrosion of steel bar with respect to orientation in concrete," ACI materials Journal, Vol. 96, No. 2, pp.154-159.

Morrthy, R.I.K., Kakodkar, A., Srirangarajan, H.R. and Suryanarayan, S. (1992). "Finite element simulation of chaotic vibrations of a beam with non-linear boundary condtions," Computers \& Structures, Vol. 49, No. 4, pp.589-596.

Munn, R.S. (1982). “A mathematical model for galvanic anode cathodic protection system," Mat. Per. Vol.21, pp.29-41.

Nakata, T., Takahashi, N., Fujiwara, K. and Okamoto, N. (1992). "Improvements of convergence characteristics of Newton-Raphson method for nonlinear magnetic field analysis," Magnetics, Vol.28, No.2, pp.1048-1051.

Neagoe, C. and Ossart, F. (1994). “Analysis of convergence in nonlinear magnetostatic finite elements problems," IEE Transactions on Magnetics, Vol. 30, No.5. pp.2865-2868.

Neville, A (1996). Properties of concrete, 4th ED, John Wiley and Sons Inc., New york, USA.

Papadakis, V., Vayenas, C.G., Fardis, M.N. (1991). "Fundametal modeling and experimental investigation of concrete carbonation," ACI Materials Journal, Vol.88, No.4, pp.363-373.

Perez, Nestor, (2004). Electrochemistry and Corrosion science, Kluwer Academic Publishers, Massachusetts, USA.

Saetta, A., Schrefler, B., Vitalini, R. (1993). "The carbonation of concrete and the mechanism of moisture, heat, carbon dioxide flow through porous materials," Cement and Concrete Research Vol.23, No.4, pp.761-772. 
Samson, E., Marchand, J., Robert, J.L., and Bournazel, J.P. (1999). "Modeling the mechanisms of ion diffusion transport in porous media," International Journal of Numerical Methods in Engineering, Vol.46, No.12, pp.2043-2060.

Sharland, S. M., Jackson, C.P. and Diver A.J. (1989). "A finite element model of the propagation of corrosion crevices and pits," Corrosion Science, Vol. 29, No. 9, pp. $1149-1166$.

Strommen, R.D. (1992). “Computer modelling of offshore cathodic protection systems: Method and Experience," Computer Modelling in Corrosion, ASTM STP 1154, Philadelphia, USA, pp.229-247.

Segerlind, Larry J. (1984). Applied finite element analysis, John Wiley \& Sons Inc, Michigan, USA.

Thomas, M.D.A., Bamforth, P.B. (1999). "Modelling chloride diffusion in concrete: effect of fly ash and slag," Cement and Concrete Research, Vol.29, pp.487-495.

Trethewey, K. R. and Chamberlain, J. (1988). Corrosion, Longman scientific \& Technical, Now York, USA.

Trethewey, K.R. and Keenan, J.S. (1992). "Microcomputer-based corrosion modelling applied to polarization curves," Computer Modeling in Corrosion, edited by X.Y. Mann, ASTM International, Philadelphia, USA, pp. 113-125.

Uhlig, H.H., Revie, R.W. (1985). Corrosion and corrosion Control, 3nd ED, J. Wiley and Sons Inc., New York, USA.

Vogelius, M. and Xu, J-M (1998). “A nonlinear elliptic boundary value problem related to corrosion modelling," Quarterly of applied mathematics, Vol.56, No.3, PP.479-505.

Walton, J., Plansky, L., and Smith, R. (1990). "Models for estimation of service life of 
concrete barriers in low-level radioactive waste disposal," U.S. Nuclear Regulatory Commission, Tech. Rep. No. NUREG/CR-5542 EGG-2597.

Yalcin, H. and Ergun, M. (1996). "The prediction of corrosion rates of reinforcing steels in concrete," Cement and Concrete Research, Vol. 26, No. 10, pp. 1593-1599.

Yokozeki, K., Motohashi, K., Okada, K., and Tsutsumi, T. (1997). “A rational model to predict the service life of RC structures in marine environment," Edited by Malhotra, V., Durability of Concrete, ACI, Detroit, USA, pp.777-799.

Zamani, N.G., Porter, J.F. and Mufti, A.A. (1986). "A survey of computational efforts in the filed of corrosion engineering," International Journal for Numerical Methods in Engineering, Vol.23, pp.1295-1311.

Zhang, J-Y, Lounis, Z. (2006). "Sensitivity analysis of simplified diffusion-based corrosion initiation model of concrete structures exposed to chlorides," Cement and Concrete Research, Vol.36, pp.1312-1323.

Zhao, J-C (2000). "Application of the direct iteration method for non-linear analysis of steel frames in fire," Fire Safety Journal, Vol.35, pp.241-255.

Zienkiewicz, O.C. (1977). The finite element method, $3^{\text {rd }}$ ED, McGRAW-HILL, London, UK. 


\section{Appendix}

\section{Developed Computer Program}

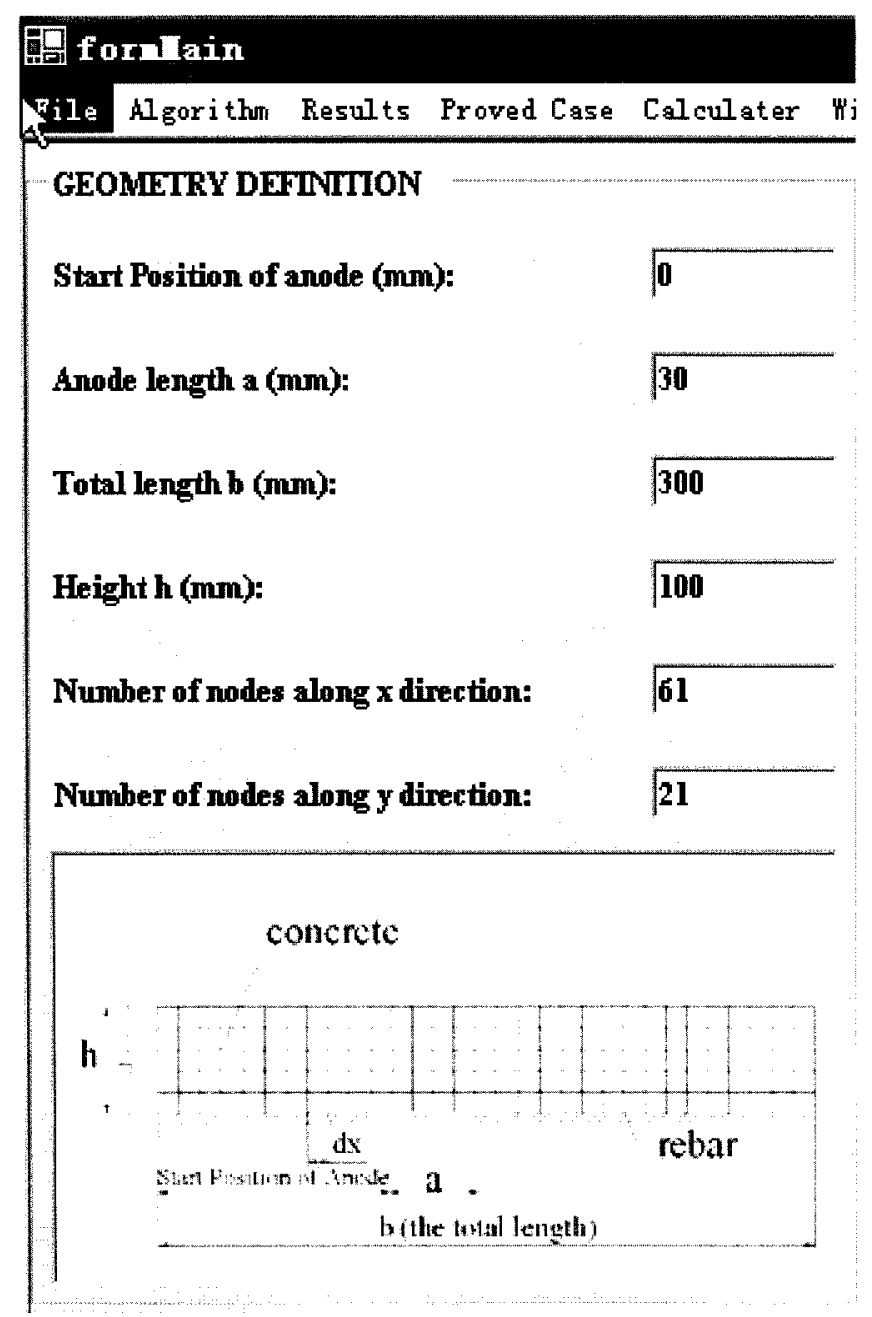

Fig. A.1: Definition of geometrical properties 


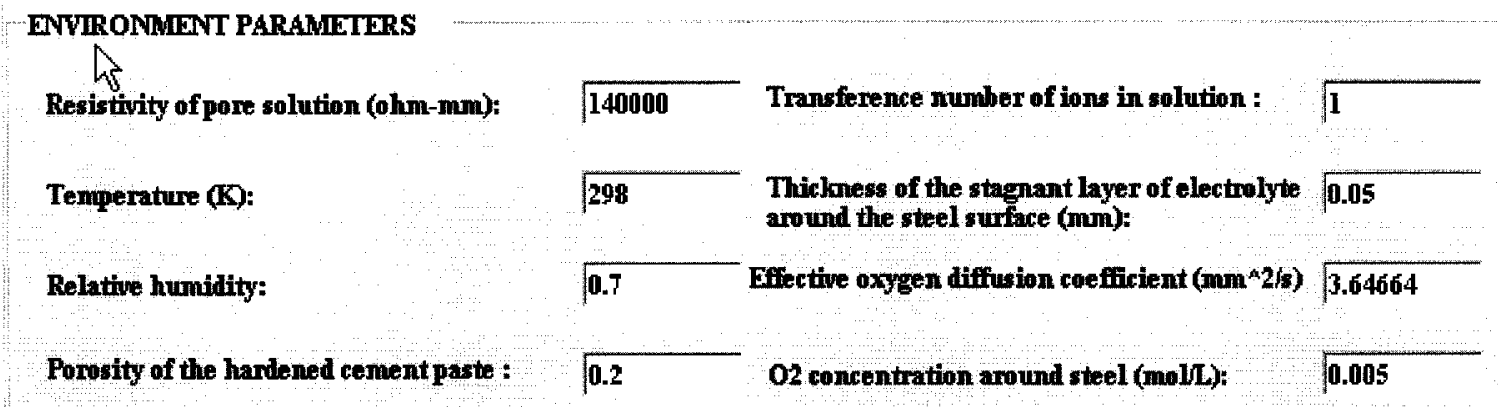

Fig. A.2: Definition of environmental parameters

\section{BOUNDARY CONDITIONS}

\section{Anode}

Standard half cell potential of Fe (mV):

$-780$

$187.5 E-12$

Anodic ex. current density (A/mm2):

Tafel slope of the anode ( $\mathrm{mV}$ ):

60

\section{Cathode}

Standard half cell potential of $02(\mathrm{mV})$

Cathodic ex. current density (A/mm2):

Tafel slope of the cathode $(\mathrm{mV})$ :

Limiting current density (A/mm2):
160

$6.25 \mathrm{E}-12$

160

1E-06

Fig. A.3: Definition of corrosion parameters that are used to calculate boundary conditions

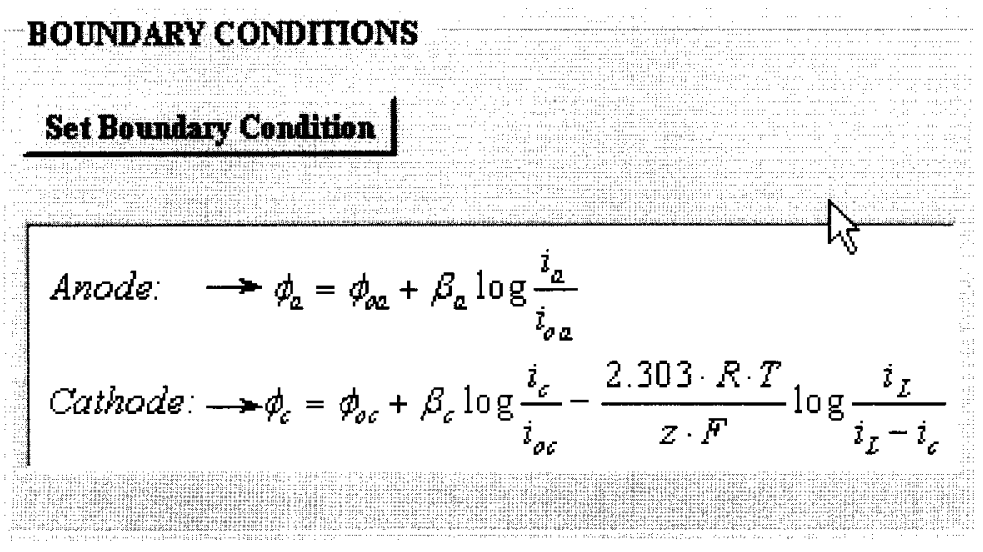

Fig. A.4: Activation of boundary condition setup 


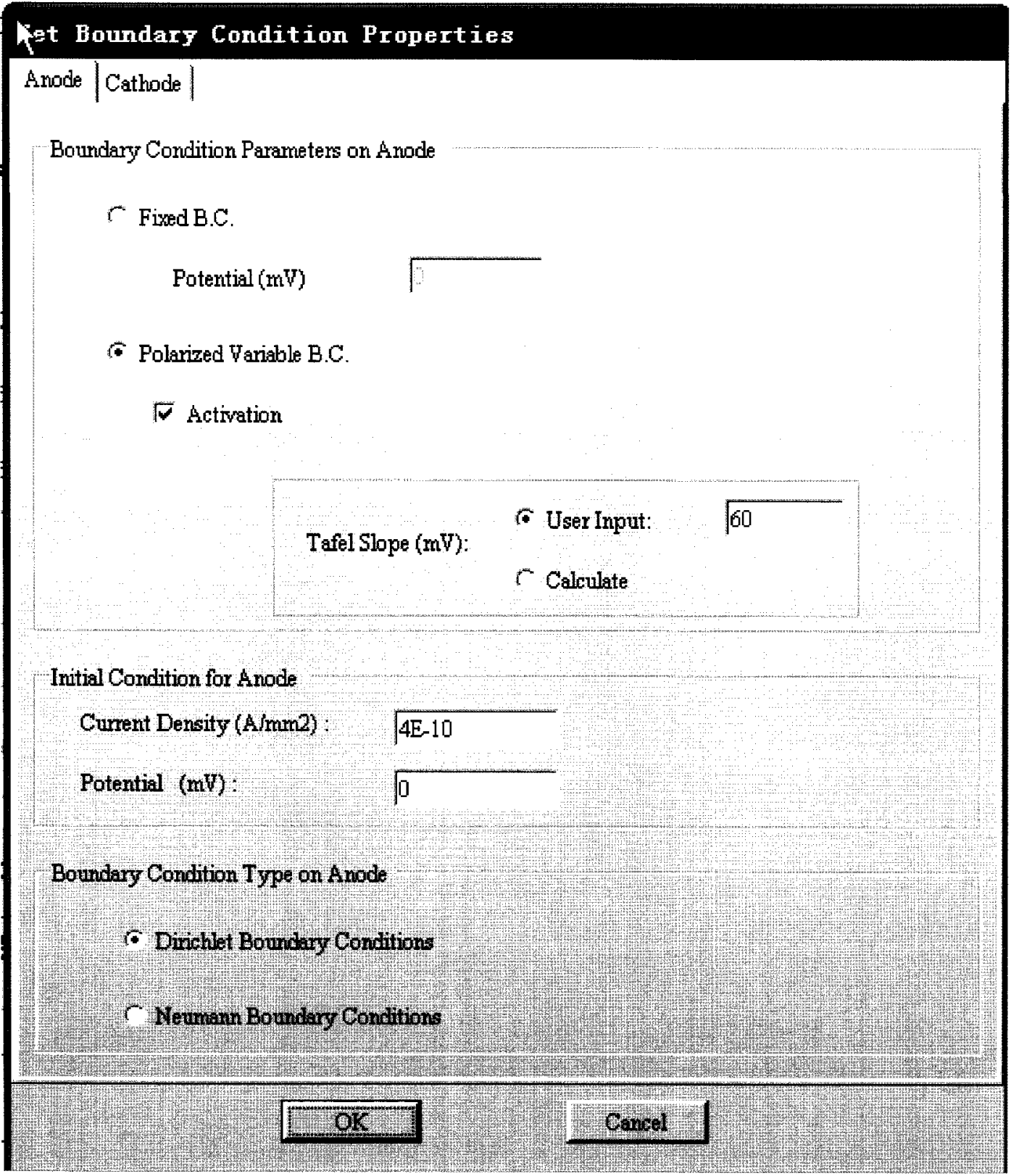

Fig. A.5: Definition of anodic boundary conditions 


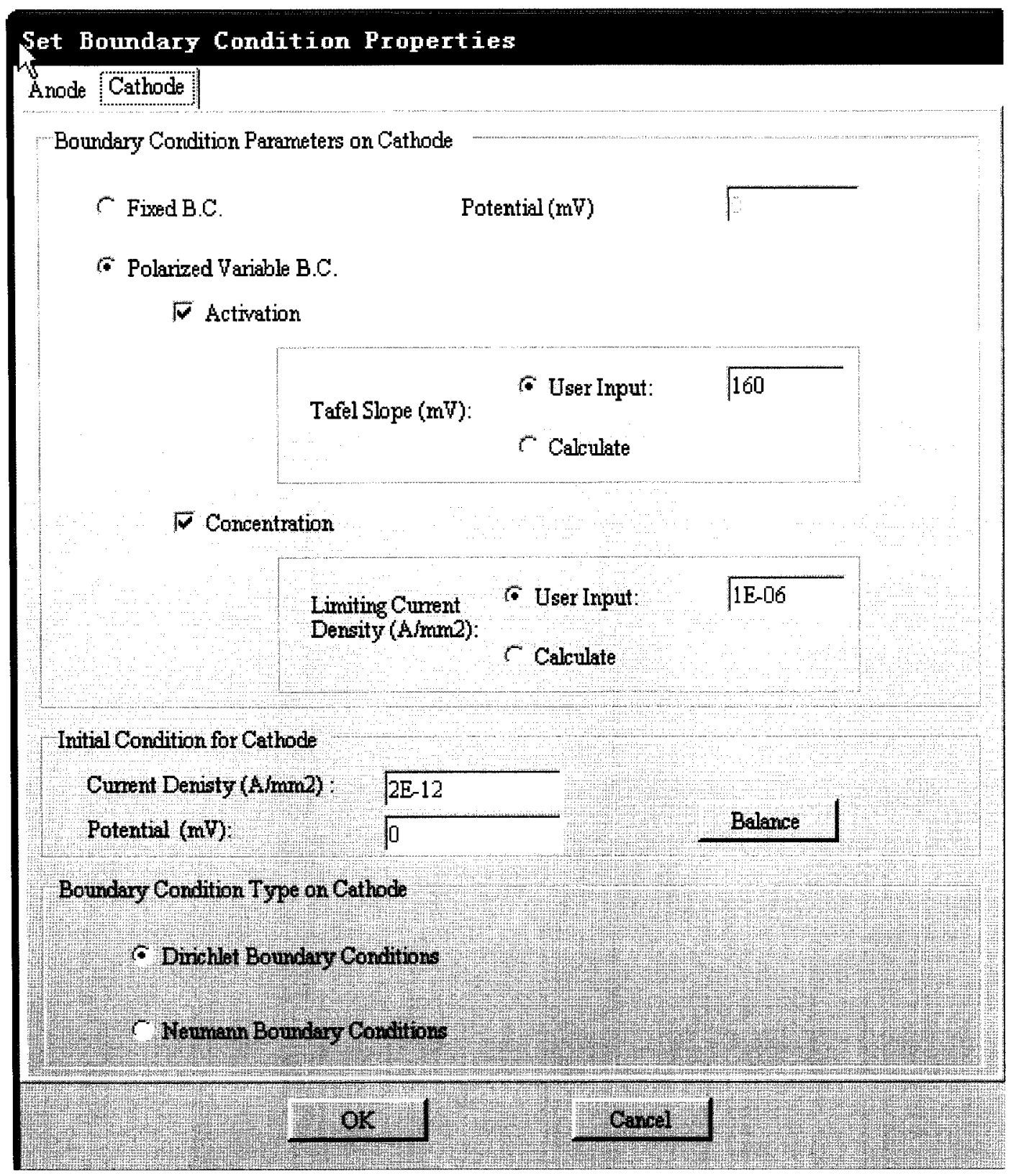

Fig. A.6: Definition of cathodic boundary conditions 


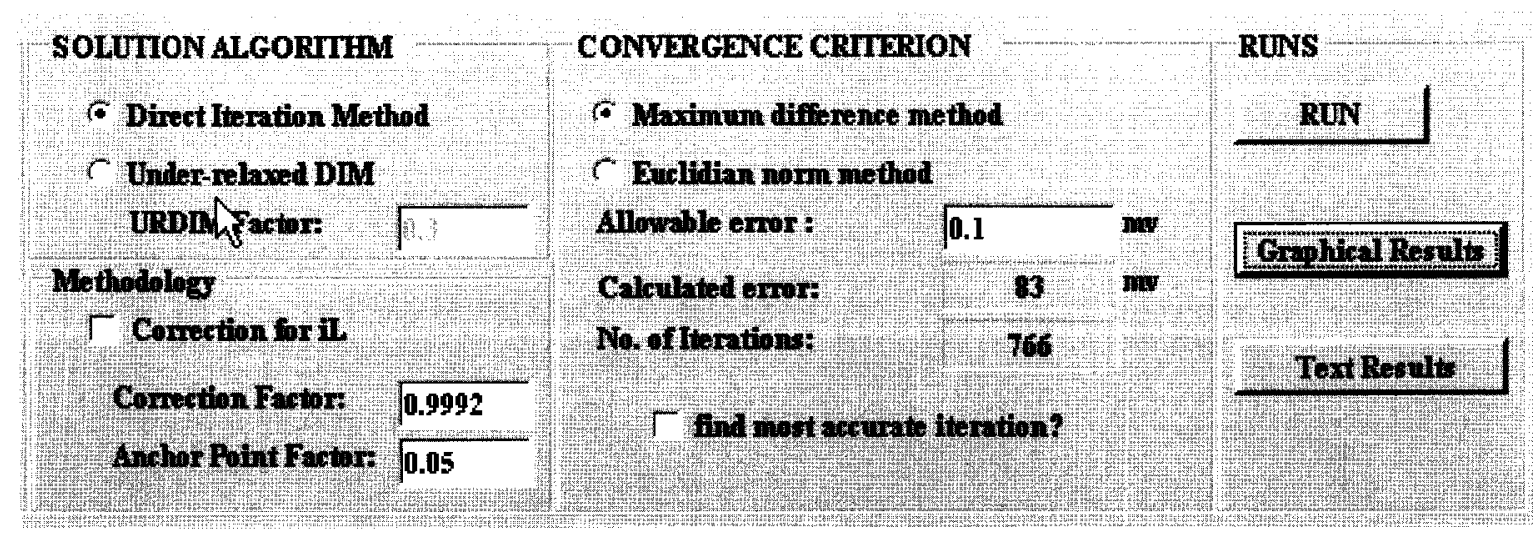

Fig. A.7: Analysis options 


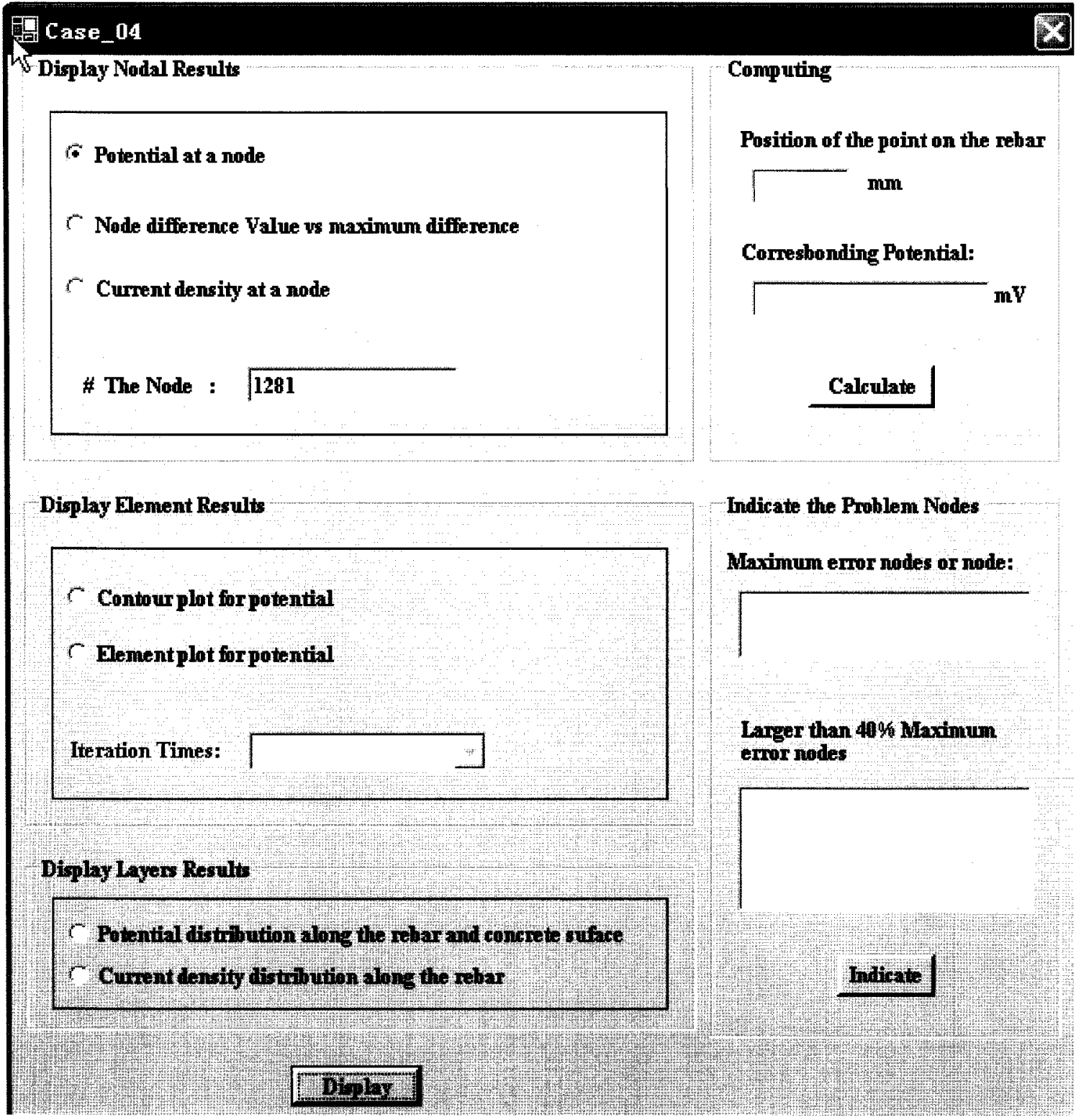

Fig. A.8: Outputting results 


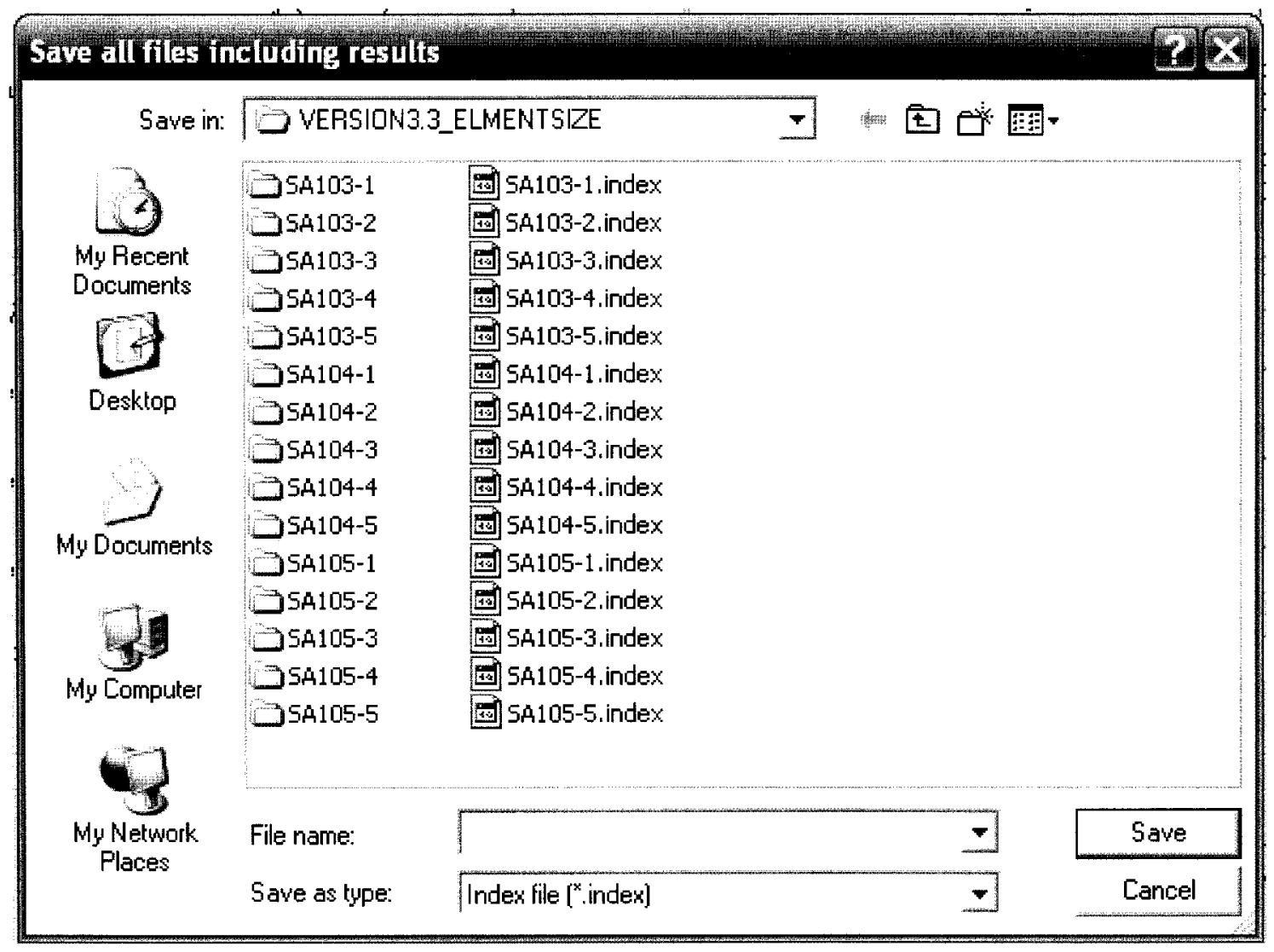

Fig. A.9: Saving data files 